\title{
Mediterranean diet and beyond
}

Citation for published version (APA):

Ruggiero, E. (2021). Mediterranean diet and beyond: an Italian perspective. [Doctoral Thesis, Maastricht University]. Maastricht University. https://doi.org/10.26481/dis.20210301er

Document status and date:

Published: 01/01/2021

DOI:

10.26481/dis.20210301er

Document Version:

Publisher's PDF, also known as Version of record

\section{Please check the document version of this publication:}

- A submitted manuscript is the version of the article upon submission and before peer-review. There can be important differences between the submitted version and the official published version of record.

People interested in the research are advised to contact the author for the final version of the publication, or visit the DOI to the publisher's website.

- The final author version and the galley proof are versions of the publication after peer review.

- The final published version features the final layout of the paper including the volume, issue and page numbers.

Link to publication

\footnotetext{
General rights rights.

- You may freely distribute the URL identifying the publication in the public portal. please follow below link for the End User Agreement:

www.umlib.nl/taverne-license

Take down policy

If you believe that this document breaches copyright please contact us at:

repository@maastrichtuniversity.nl

providing details and we will investigate your claim.
}

Copyright and moral rights for the publications made accessible in the public portal are retained by the authors and/or other copyright owners and it is a condition of accessing publications that users recognise and abide by the legal requirements associated with these

- Users may download and print one copy of any publication from the public portal for the purpose of private study or research.

- You may not further distribute the material or use it for any profit-making activity or commercial gain

If the publication is distributed under the terms of Article $25 \mathrm{fa}$ of the Dutch Copyright Act, indicated by the "Taverne" license above, 
(C) Copyright: Emilia Ruggiero, Maastricht 2021

Thesis Maastricht University

Layout: Emilia Ruggiero

Cover: Renato Guttuso, Vucciria, 1974

Printed by: Cicchetti Industrie Grafiche - Isernia, Italy

ISBN: 979-12-200-8084-2

The studies presented in this thesis were conducted at the IRCCS Neuromed, the Department of Epidemiology and Prevention, Pozzilli, Italy 


\section{DISSERTATION}

to obtain the degree of Doctor at Maastricht University on the authority of the Rector Magnificus,

Prof. Dr. Rianne M. Letschert

in accordance with the decision of the Board of Deans,

to be defended in public

on Monday 1 March 2021, at 13:00 hours

by

Emilia Ruggiero

Approved, after corrections

Prof.dr. Rianne M. Letschert

Rector Magnificus 


\section{Supervisors}

Prof. dr. Hugo ten Cate

Prof. dr. Giovanni de Gaetano, Pozzilli, Italy

\section{Co-supervisors}

Prof. dr. Licia Iacoviello, Pozzilli and Varese -Como, Italy

Dr. Marialaura Bonaccio, Pozzilli, Italy

\section{Assessment Committee}

Prof. dr. Manuela Joore, (Chair)

Dr. ir Yvonne Henskens

Prof. dr. Monika Stoll

Prof. dr. Saverio Stranges, Western University, Canada

Prof. dr. Francesco Sofi, University of Florence, Italy 
To my family 

Chapter 1

pag. 9

General Introduction

Chapter 2

21

Food group consumption in an Italian population using the updated food classification system FoodEx2: Results from the Italian Nutrition \& HEalth Survey (INHES) study

Chapter 3

45

Consumption of whole grain food and its determinants in a general Italian population: Results from the INHES study

\section{Chapter 4}

Socioeconomic and psychosocial determinants of adherence to the

Mediterranean diet in a general adult Italian population

\section{Chapter 5}

Fish intake is associated with lower cardiovascular risk in a Mediterranean population: prospective results from the Moli-sani study

\section{Chapter 6}

Egg consumption and risk of total and cause-specific mortality in a healthy Mediterranean population

\section{Chapter 7}

Chili Pepper Consumption and Mortality in Italian Adults

\section{Chapter 8}

Daily coffee drinking is associated with lower risks of cardiovascular and total mortality in a general Italian population: results from the Moli-sani Study

\section{Chapter 9}

General Discussion

Chapter 10

Valorization

Chapter 11

General Conclusions 
Chapter 12

Summary

Chapter 13

161

Candidate's Curriculum vitae

Chapter 14

163

Candidate's Publications

Chapter 15

Acknowledgements 
In the Fifties, the American scientist Ancel Keys from the University of Minnesota, described the eating habits of countrymen in Southern Italy in this way: “... homemade minestrone, pasta of all varieties, with tomato sauce and a sprinkling of Parmesan, only occasionally enriched with a few pieces of meat or served with a small fish of the place beans and macaroni ..., so much bread, never removed from the oven more than a few hours before being eaten, and nothing with which spread it, lots of fresh vegetables sprinkled with olive oil, a small portion of meat or fish maybe a couple of times a week and always fresh fruit for dessert ". This was the first description of the Mediterranean way of eating (1), later on referred to as the Mediterranean Diet (2).

Even today, the Mediterranean diet is considered the golden standard of healthy nutrition and is described as the traditional dietary pattern of the olive tree-growing areas of the Mediterranean basin, characterized by high intake of plant foods (cereals, fruits, vegetables, legumes, tree nuts, seeds and olives), olive oil as the principal source of added fat, high to moderate intakes of fish and seafood, moderate consumption of eggs, poultry and dairy products (cheese and yoghurt), low consumption of red meat and moderate intake of alcohol (mainly wine) during meals (2).

On 16 November 2010, UNESCO approved the inscription of the Mediterranean Diet in the Representative List of the Intangible Cultural Heritage of Humanity summarizing it as follows: "The Mediterranean Diet constitutes a set of skills, knowledge, practices and traditions ranging from the landscape to the table, including the crops, harvesting, fishing, conservation, processing, preparation and, particularly, consumption of food. The Mediterranean Diet is characterized by a nutritional model that has remained constant over time and space, consisting mainly of olive oil, cereals, fresh or dried fruit and vegetables, a moderate amount of fish, dairy and meat, and many condiments and spices, all accompanied by wine or infusions, always respecting the beliefs of each community" (3).

The healthy traditional Mediterranean dietary pattern has been popularized through a pyramid representation at the base of which are placed foods that should sustain the diet, while at the upper levels, foods to be eaten in moderate/low amounts.

The health benefits associated with the Mediterranean diet have been well established by several epidemiological studies consistently showing that adherence to this dietary pattern is 
associated with a significant reduction in the risk of developing cardiovascular disease, cancer and neurodegenerative diseases as well as all-cause mortality (4).

Nevertheless, late evidence has been suggesting a global shifting in Mediterranean societies that are rapidly withdrawing from this eating pattern rather orienting their food choices towards products typical of the Western diet pattern, an unhealthy eating behavior characterized by high intake of nutrient-poor foods, refined grains, animal fats, sugars, processed meat but poor in legumes, cereals, fruits and vegetables (5).

In light of this, it is crucial to provide an estimation of the actual adherence to the Mediterranean diet among Mediterranean populations, also by offering insights on the main determinants of dietary habits.

As discussed in a recent commentary by Jeremiah Stamler, one of the pioneer researchers of the Mediterranean diet and trusty collaborator of Ancel Keys, several things have changed since the original definition of the Mediterranean diet and many problematic nutritional aspects emerged over the last decades (6). The high total energy and salt intake, greater red meat consumption are just some examples of how modern dietary habits shifted from the traditional dietary pattern.

Thus, it is particularly important to re-organize the major food groups presently included in the Italian diet in order to evaluate which are the most consumed foods and their determinants. Among them, a crucial role is played by grain products, that nowadays are almost exclusively from white flour (not whole grain flour) and whose high intake has been associated with increased risk of diabetes (7) and metabolic syndrome (8).

The Italian Nutrition \& Health Survey (INHES) has offered the most up-to-date evidence on the consumption trends of major food groups consumed in Italy between 2010-2013, and also provided detailed information on major determinants associated with dietary habits, meal patterns and other factors possibly influencing diet.

In epidemiological studies, adherence to a given diet is commonly measured by a-priori dietary scores including a variable number of food groups or nutrients.

The theoretical advantage of studying a dietary pattern characterized by fewer food groups is that it more readily lends itself to application across populations.

The 'a priori-defined' approach is based on predefined diet quality indices using current nutrition knowledge about the relationships between food and disease (like the food pyramids). This approach is not necessarily wrong (on the contrary in most of the cases 
reflects accurate information from series of valid studies), but shares the limitations of "static" evaluation that cannot incorporate actual particularities of individuals' choices in relation to the development of a disease. Also, the a-priori approaches quantitatively measure the features of dietary habits that already have been recommended as 'healthy'.

Along, with a-priori dietary scores, nutritional epidemiology also relies on the use of $a$ posteriori approaches also known as "empirically, or data-driven, derived dietary patterns" (9).

One key advantage of the latter approach is that it takes into account many aspects of the diet rather than focusing on a few hypothesized key food groups. Furthermore, food groups used in most a-priori scores are usually broadly defined and do not take into account subtle differences in the nutrients and phytochemicals in individual food items.

Since the a posteriori approaches (e.g. factor analysis) produce dietary patterns on the basis of available empirical data without any a-priori hypothesis, they might not necessarily represent optimal dietary patterns (10).

Several scores are used to measure the adherence to the Mediterranean diet. The most widely used is the one conceived by Trichopoulou and colleagues (11). The Mediterranean Diet Score is the most extensively used index due to its ease of application, and many variants have been created for the evaluation of multiple diet-health relationships.

The traditional Greek Mediterranean Diet (MDS-1) (12) originally comprised 8 food groups: 1) High ratio of monounsaturated: saturated fat; 2) Moderate alcohol intake; 3) High legume intake; 4) High intake of grains including bread and potatoes); 5) High fruit intake; 6) High vegetable intake; 7) Low intake of meat and meat products and 8) Moderate intake of milk and dairy products. The MDS-1 was based on assigning a score from 0 to 1 according to the daily intake of the eight components. In general, the medians of the sample, specific for sex, were used as cut-off points and grams per day were used as the intake measurement. A subject received a point if his intake was over the sample median for a protective component (vegetables, fruits, etc.) and below the median for non-protective components (dairy products, meat, etc.). In the case of alcohol (except when specified) 1 point was scored for males if their consumption was within 10 and $50 \mathrm{~g} /$ day, and within 5 and $25 \mathrm{~g} /$ day for women. If all the characteristics of the diet were incorporated, the highest score was obtained and reflected a greater adherence to the MD. Therefore, the MDS- 1 usually ranged from 0 (minimal adherence) to 8 (maximum adherence) if the index had eight components. 
Generally, a score of 4 or more was associated with satisfactory MD adherence and better health implications.

In 2003, another prospective investigation by the same group of Authors analyzed a population of 22,043 Greek adults, 20-86 years old, during a median of 44 months of followup (16). In this case, adherence to the traditional Mediterranean diet was assessed by the same scale developed by Trichopoulou et al. (1995) (12), but revised to include fish intake.

The addition to the Mediterranean diet score of a ninth component incorporating fish intake was deemed necessary not only because fish is an important part of the Mediterranean diet, but also because of strong evidence of an inverse correlation between fish consumption and the risk of death from coronary heart disease (13).

Due to the central importance of fish intake in the diet, and the paucity of data from Mediterranean populations, one of the papers included in this thesis (Chapter 5) addressed the role of this food towards the risk of developing CVD.

The Mediterranean Diet Score, or its modified versions, has been proven to be predictive of disease/mortality risk also in non-Mediterranean populations (14-16), and sometimes resulted as the best performing dietary model as compared to other healthy dietary patterns (17-19).

Along with the Greek Mediterranean Diet Score, a number of other indices were developed and many of them were conceptualized in Mediterranean countries: 1) In Greece the Mediterranean diet score proposed by Panagiotakos and colleagues (20); 2) In Italy Mediterranean Adequacy Index (MAI) by Alberti-Fidanza and colleagues (21); 3) Sofi and colleagues proposed a literature-based adherence score (22); 4) in EPICOR a new Italian Mediterranean Index developed by the authors (23) by adapting the Greek Mediterranean Index to the Italian eating behavior, with emphasis on EVOO and pasta intake.

Although including a large number of foods typical of the Mediterranean diet, the most common dietary indices measuring the Mediterranean diet do not provide any indication for some other widely consumed foods (e.g. eggs, potatoes) or beverages, such as coffee, nor quantify the use of spices which are an integral part of the Mediterranean diet.

In light of the above, it will be of interest to analyze whether foods and beverages, some already well-known for their health advantages (e.g. coffee), not included in the Mediterranean Diet Score, nor in the majority of its modified versions, are associated with health outcomes in a population with typical Mediterranean dietary habits, and also to test 
whether the inclusion of these foods and beverages might improve risk prediction beyond a traditional Mediterranean Diet Score.

In order to fulfill the different purposes of this thesis, two large datasets were used; the Italian Nutrition \& Health Survey (INHES) offered the opportunity to determine the actual dietary trends of consumption of major food groups and identify the main dietary patterns in Italy along with their demographic and socioeconomic determinants.

The second source of data is represented by the large population-based cohort of the Molisani Study, which allowed to address longitudinally the relation between diet and food items with risk of chronic disease or mortality.

The use of integrated data sources at national level (INHES Study), and from a representative Italian Mediterranean region (Moli-sani Study) offers a unique opportunity to obtain a complete and informative Italian perspective on diet and surroundings from which to confirm or to plan novel public health actions.

Therefore, the aims of this thesis are the following:

1) to re-organize the major food groups presently included in the Italian diet;

2) to evaluate the adherence to the Mediterranean Diet in Italy and its major socioeconomic and demographic determinants;

3) to address the health effects possibly associated with fish intake;

4) to evaluate the association with mortality of largely consumed foods (chili pepper and eggs) and beverages (coffee) that are not included in the traditional Mediterranean Diet Score, in a large sample of the Italian population recruited in the Moli-sani Study cohort;

5) to verify whether the inclusion of some of these foods/beverages into the Mediterranean Diet Score will improve its risk prediction.

\section{Assessment of food groups included in the Italian diet}

The first paper here presented (Chapter 2) contains updated information on food group consumption in Italy by using data from the Italian Nutrition \& Health Survey (INHES). The last suitable Italian public health surveillance program on nutrition has been held by the INRAN (National Institute for Research on Foods and Nutrition) covering the years 2005-06 (24). Since then, no further information has been gathered at the national level for food intake all over Italy. 
The INHES study is a 3-year telephone-based survey on nutrition and health specifically designed to collect information on the dietary habits (quality, quantity and patterns), food choice determinants and food health awareness of the Italian population according to different geographical distribution (Northern, Central and Southern Italy), age, gender and socioeconomic profile. Between November 2010 and November 2013, 9,319 women and men aged $\geq 5$ years from all over Italy were enrolled.

This paper identified over 70 food groups that were analyzed by gender, age group, geographical area, educational level or type of meal (e.g. breakfast, lunch).

\section{Whole grain as a key component of the Mediterranean diet pyramid}

Chapter 3 reports data on whole grain food intake and also addresses its major demographic, socioeconomic, psychosocial and behavioral determinants in the general Italian population of adolescents and adults recruited in the INHES Survey.

The Mediterranean diet pyramid recommends the consumption of one or two servings of cereals at each main meal (in the form of bread, pasta, rice, couscous and others), preferably whole grain (25).

The latest updated data on whole grain consumption in Italy derive from the INRAN-SCAI 2005-06, a cross-sectional survey conducted on a representative sample of 1300 households randomly selected and stratified into the four main geographical areas of Italy (North-West, North-East, Centre, South and Islands). This study showed that whole grain products, over the 3-day survey period, were consumed in only a quarter of Italian children and adults, with mean intakes being substantially lower than quantitative whole grain recommendations (26). Countries including the USA, Canada, Denmark and Australia have specific dietary guidelines on whole grain intake but others, as Italy, do not explicitly indicate the amount of whole grain to be consumed, rather they only suggest to regularly consume cereals preferably as whole grain.

\section{Determinants of adherence to the Mediterranean diet}

The third paper of the thesis (Chapter 4) investigated the actual adherence to the Mediterranean diet in the adult population recruited from the INHES study and discusses its major demographic, socioeconomic, psychosocial and behavioral factors.

More recently, a global shifting from the Mediterranean diet was documented especially in the Mediterranean regions that experienced the greatest loss of adherence as opposed to non- 
Mediterranean Countries (27), to such an extent that a nutrition transition issue emerged also for Mediterranean populations (28).

In light of the above, improving the understanding of main determinants of the Mediterranean diet adherence during the 21st century is of crucial importance in order to develop effective public health strategies for prevention of major chronic diseases, especially at a time of financial crisis during which efforts and resources should be strategically delivered.

An additional key component of the bundle of foods included in the Mediterranean diet pyramid is fish, that was only added to the final version of the Mediterranean Diet Score, in 2003. However, little is known on the association of fish and cardiovascular risk within Mediterranean population settings, and available evidence dates back to men recruited in the Italian segment of the Seven Countries Study (29).

The fourth paper of this thesis (Chapter 5) reports the association between fish intake and risk of composite coronary heart disease (CHD) and cerebrovascular disease in the Moli-sani Study, a large population-based cohort of 24,325 men and women aged $\geq 35$ years living in a Southern Italian region. In this paper a first attempt has been made to evaluate the healthy benefit of processed fish.

\section{Beyond the traditional Mediterranean diet: foods, beverages and spices not included in the Mediterranean Diet Score}

Generally, the following foods and beverages are not considered when assessing the adherence to the Mediterranean diet in epidemiological studies.

Eggs are a very common food in our diet, they contain are an inexpensive source of high quality protein, all B vitamins, folate, fat-soluble vitamins, as well as, several essential minerals that play a fundamental role in basic nutrition. On the other hand, eggs are a main source of cholesterol and relevant of saturated fat that are potentially associated with an increased risk of cardiovascular diseases and type 2 diabetes (30).

Spices, included chili pepper, have long been an essential part of the traditional Mediterranean diet, and are placed, along with herbs, at the base of the Mediterranean diet pyramid both for their nutritional properties and as a valuable substitute for salt (31). Health benefits of chili peppers have been ascribed to capsaicin, its major pungent compound, which has been observed to favorably improve cardiovascular function and metabolic regulation in experimental and population studies (33). 
Coffee is one of the most commonly consumed non-alcoholic beverages worldwide. In general, the association between coffee consumption and a variety of conditions and diseases has been examined extensively, often with contrasting results. Although some studies reported that coffee consumption was associated with higher mortality in general population (35), most published studies have shown that regular coffee consumption is associated with a lower incidence of diabetes (34) and certain types of cancers (35), and with a lower total and CVD mortality $(36,37)$.

Chapters 6 and 7 investigated the association of eggs and chilies with mortality risk in a large Mediterranean population of adult men and women, and assessed whether inclusion of chili pepper is able to improve risk prediction associated with a traditional Mediterranean Diet Score.

Finally, Chapter 8 analyzed the association of coffee, and also tested whether inclusion of coffee intake would improve risk prediction beyond the traditional Mediterranean Diet Score. These last studies help clarify the health contribution of foods and beverages largely consumed by the Italian population that are not included in a traditional Mediterranean diet score.

\section{LIST OF PAPERS INCLUDED IN THIS THESIS}

1. Pounis G, Bonanni A, Ruggiero E, Di Castelnuovo A, Costanzo S, Persichillo M, Bonaccio M, Cerletti C, Riccardi G, Donati MB, de Gaetano G, Iacoviello L; INHES Investigators. Food group consumption in an Italian population using the updated food classification system FoodEx2: Results from the Italian Nutrition \& HEalth Survey (INHES) study. Nutr Metab Cardiovasc Dis. 2017 Apr; 27(4):307-328.

2. Ruggiero E, Di Castelnuovo A, Costanzo S, Persichillo M, Bracone F, Cerletti C, Donati MB, de Gaetano G, Iacoviello L, Bonaccio M; INHES Study Investigators. Socioeconomic and psychosocial determinants of adherence to the Mediterranean diet in a general adult Italian population. Eur J Public Health. 2018 Jul 17.

3. Bonaccio M, Ruggiero E, Di Castelnuovo A, Costanzo S, Persichillo M, De Curtis A, Cerletti C, Donati MB, de Gaetano G, Iacoviello L. on behalf of the Moli-sani study Investigators. Fish intake is associated with lower cardiovascular risk in a Mediterranean population: prospective results from the Moli-sani study. 
Nutr Metab Cardiovasc Dis. 2017 Oct;27(10):865-873.

4. Ruggiero E, Bonaccio M, Di Castelnuovo A, Bonanni A, Costanzo S, Persichillo M, Bracone F, Cerletti C, Donati MB, de Gaetano G, Iacoviello L; INHES Study Investigators. Consumption of whole grain food and its determinants in a general Italian population: Results from the INHES study. Nutr Metab Cardiovasc Dis. 2019 Jun;29(6):611-620.

5. Bonaccio M, Di Castelnuovo A, Costanzo S, Ruggiero E, De Curtis A, Persichillo M, Tabolacci C, Facchiano F, Cerletti C, Donati MB, de Gaetano G, Iacoviello L; Moli-sani Study Investigators. Chili Pepper Consumption and Mortality in Italian Adults. J Am Coll Cardiol. 2019 Dec 24;74(25):3139-3149.

6. Ruggiero E, Di Castelnuovo A, Costanzo S, Persichillo M, De Curtis A, Cerletti C, Donati MB, de Gaetano G, Iacoviello L, Bonaccio M, on behalf of the Moli-sani study Investigators. Egg consumption and risk of total and cause-specific mortality in a healthy Mediterranean population. Submitted

7. Ruggiero E, Di Castelnuovo A, Costanzo S, Persichillo M, De Curtis A, Cerletti C, Donati MB, de Gaetano G, Iacoviello L, Bonaccio M, on behalf of the Moli-sani Study Investigators. Daily coffee drinking is associated with lower risks of cardiovascular and total mortality in a general Italian population: results from the Moli-sani Study. J Nutr. 2020 Dec 31; nxaa365. 
1. Ancel and Margaret Keys. HOW TO EAT WELL AND STAY WELL: THE MEDITERRANEAN WAY. New York: Doubleday; 1975

2. Willett WC, Sacks F, Trichopoulou A, et al. Mediterranean diet pyramid: a cultural model for healthy eating. Am J Clin Nutr 1995;61(6 Suppl.):1402Se6S

3. https://ich.unesco.org/en/RL/mediterranean-diet-00884

4. Sofi F, Macchi C, Abbate R, et al. Mediterranean diet and health status: an updated meta-analysis and a proposal for a literature-based adherence score. Public Health Nutr. 2014 Dec;17(12):2769-82.

5. Bonaccio M, Di Castelnuovo A, Bonanni A, et al. Decline of the Mediterranean diet at a time of economic crisis. Results from the Moli-sani study. Nutrition Metabolism and Cardiovascular disease. 2014;24:853-60.

6. Stamler J. Toward a modern Mediterranean diet for the 21 st century. Nutr Metab Cardiovasc Dis. 2013;23:1159-62.

7. Hu FB. Globalization of diabetes: The role of diet, lifestyle, and genes Diabetes Care, 34 (6) (2011), pp. 1249-1257

8. Song S, Lee JE, Song WO et al. Carbohydrate intake and refined-grain consumption are associated with metabolic syndrome in the Korean adult population. J Acad Nutr Diet. 2014 Jan;114(1):54-62.

9. Meropi D. Kontogianni, Demosthenes B. Panagiotakos. Dietary patterns and stroke: A systematic review and re-meta-analysis. Maturitas. 2014 Sep;79(1):41-7.

10. Hoffmann K, Schulze MB, Schienkiewitz A, et al. Application of a new statistical method to derive dietary patterns in nutritional epidemiology. Am J Epidemiol. 2004 May 15;159(10):935-44.

11. Trichopoulou A, Costacou T, Bamia C, Trichopoulos D. Adherence to a Mediterranean diet and survival in a Greek population. N Engl J Med 2003;348:2599-2608.

12. Trichopoulou A, Kouris-Blazos A, Wahlqvist ML, et al. Diet and overall survival in elderly people. BMJ. 1995 Dec 2;311(7018):1457-60.

13. Hu FB, Bronner L, Willett WC, et al. Fish and omega-3 fatty acid intake and risk of coronary heart disease in women. JAMA 2002;287:1815-1821 
14. Tektonidis TG, Åkesson A, Gigante B, et al. A Mediterranean diet and risk of myocardial infarction, heart failure and stroke: A population-based cohort study. Atherosclerosis. 2015;243:93-8.

15. Tong TY, Wareham NJ, Khaw KT, et al. Prospective association of the Mediterranean diet with cardiovascular disease incidence and mortality and its population impact in a non-Mediterranean population: the EPIC-Norfolk study. BMC Med. 2016 Sep 29;14(1):135.

16. Whalen KA, McCullough M, Flanders WD, et al. Palaeolithic and Mediterranean diet pattern scores and risk of incident, sporadic colorectal adenomas. Am J Epidemiol. 2014;180:1088-97

17. Whalen KA, Judd S, McCullough ML, et al. Palaeolithic and Mediterranean Diet Pattern Scores Are Inversely Associated with All-Cause and Cause-Specific Mortality in Adults. J Nutr. 2017;147:612-620.

18. Warensjö Lemming E, Byberg L, Wolk A, Michaëlsson K. A comparison between two healthy diet scores, the modified Mediterranean diet score and the Healthy Nordic Food Index, in relation to all-cause and cause-specific mortality. Br J Nutr. 2018;119:836-846.

19. Ratjen I, Schafmayer C, di Giuseppe R, et al. Postdiagnostic Mediterranean and Healthy Nordic Dietary Patterns Are Inversely Associated with All-Cause Mortality in LongTerm Colorectal Cancer Survivors. J Nutr. 2017;147:636-644.

20. Panagiotakos DB, Pitsavos C, Stefanadis C. Dietary patterns: a Mediterranean diet score and its relation to clinical and biological markers of cardiovascular disease risk. Nutr Metab Cardiovasc Dis 2006;16:559-68.

21. Alberti-Fidanza A, Fidanza F. Mediterranean Adequacy Index of Italian diets. Public Health Nutr 2004;7:937-41.

22. Sofi F, Macchi C, Abbate R, et al. Mediterranean diet and health status: an updated meta-analysis and a proposal for a literature based adherence score. Public Health Nutr 2014;17:2769-82.

23. Agnoli C, Krogh V, Grioni S, et al. A priori-defined dietary patterns are associated with reduced risk of stroke in a large Italian cohort. J Nutr. 2011 Aug;141(8):1552-8.

24. Sette S, Le Donne C, Piccinelli R, et al. The third Italian National Food Consumption Survey, INRAN-SCAI 2005-06-part 1: nutrient intakes in Italy. Nutr Metab Cardiovasc Dis 2011;21:922e32. 
25.http://www.salute.gov.it/imgs/C_17_pubblicazioni_652_allegato.pdf.

26. Sette S, D'Addezio L, Piccinelli R, et al. Intakes of whole grain in an Italian sample of children, adolescents and adults. Eur J Nutr 2017;56(2):521e33.

27. Leo'n-Muñz LM, Guallar-Castillo'n P, Graciani A, et al. Adherence to the Mediterranean diet pattern has declined in Spanish adults. J Nutr 2012;142:1843-50.

28. Bonaccio M, Bes-Rastrollo M, de Gaetano G, Iacoviello L. Challenges to the Mediterranean diet at a time of economic crisis. Nutr Metab Cardiovasc Dis 2016;26:1057-63.

29. Oomen CM, Feskens EJ, Räsänen L, et al. Fish consumption and coronary heart disease mortality in Finland, Italy, and The Netherlands. Am J Epidemiol 2000;151:999e1006.

30. Clayton ZS, Fusco E, Kern M (2017) Egg consumption and heart health: a review. Nutrition. 2017 May;37:79-85.

31. Martínez-González MÁ, Hershey MS, Zazpe I, Trichopoulou A. Transferability of the Mediterranean Diet to Non-Mediterranean Countries. What Is and What Is Not the Mediterranean Diet.Nutrients. 2017 Nov 8;9(11).

32. Sun F, Xiong S, Zhu Z. Dietary Capsaicin Protects Cardiometabolic Organs from Dysfunction. Nutrients. 2016 Apr 25;8(5).

33. Liu J, Sui X, Lavie CJ, et al. Association of coffee consumption with all-cause and cardiovascular disease mortality. Mayo Clin Proc. 2013 Oct;88(10):1066-74.

34. Ding M, Bhupathiraju SN, Chen M, et al. Caffeinated and decaffeinated coffee consumption and risk of type 2 diabetes: a systematic review and a dose-response meta-analysis. Diabetes Care. 2014 Feb;37(2):569-86.

35. Caini S, Cattaruzza S, Bendinelli B, et al. Coffee, tea and caffeine intake and the risk of non-melanoma skin cancer: a review of the literature and meta-analysis. Eur J Nutr. 2017 Feb;56(1):1-12.

36. Grosso G, Stepaniak U, Micek A, et al. Coffee consumption and mortality in three Eastern European countries: results from the HAPIEE (Health, Alcohol and Psychosocial factors In Eastern Europe) study. Public Health Nutr. 2017 Jan;20(1):8291.

37. Malerba S, Turati F, Galeone C, et al. A meta-analysis of prospective studies of coffee consumption and mortality for all causes, cancers and cardiovascular diseases. Eur J Epidemiol. 2013 Jul;28(7):527-39. 


\section{CHAPTER 2.}

Food group consumption in an Italian population using the updated food classification system FoodEx2: Results from the Italian Nutrition \& HEalth Survey (INHES) study

Pounis G, Bonanni A, Ruggiero E, Di Castelnuovo A, Costanzo S, Persichillo M, Bonaccio M, Cerletti C, Riccardi G, Donati MB, de Gaetano G, Iacoviello L; INHES Investigators

Nutr Metab Cardiovasc Dis. 2017 Apr; 27(4):307-328. 



\title{
Food group consumption in an Italian population using the updated food classification system FoodEx2: Results from the Italian Nutrition \& HEalth Survey (INHES) study
}

\author{
G. Pounis ${ }^{\text {a }}$, A. Bonanni ${ }^{\text {a }}$, E. Ruggiero ${ }^{\text {a }}$, A. Di Castelnuovo ${ }^{\text {a }}$, S. Costanzo ${ }^{\text {a }}$, \\ M. Persichillo ${ }^{\text {a }}$, M. Bonaccio ${ }^{a}$, C. Cerletti ${ }^{\text {, }}$, G. Riccardi ${ }^{\text {b }}$, M.B. Donati ${ }^{\text {a }}$, G. de Gaetano ${ }^{\text {a }}$, \\ L. Iacoviello ${ }^{a, *}$ on behalf of the INHES Investigators \\ ${ }^{a}$ Department of Epidemiology and Prevention, IRCCS Istituto Neurologico Mediterraneo Neuromed, Pozzilli (IS), Italy \\ ${ }^{b}$ Department of Clinical Medicine and Surgery, Federico II University, Naples, Italy \\ Received 31 August 2016; received in revised form 5 January 2017; accepted 7 January 2017 \\ Available online 18 January 2017
}

\section{KEYWORDS \\ Food; \\ Dietary pattern; \\ Dietary habits; \\ FoodEx2; \\ Food classification \\ system}

\begin{abstract}
Background and Aim: Dietary habits evolve over time, being influenced by many factors and complex interactions. This work aimed at evaluating the updated information on food group consumption in Italy.

Methods and Results: A total of 8944 (4768 women and 4176 men) participants aged $>18$ years from all over Italy recruited in 2010-13 (Italian Nutrition \& HEalth Survey, INHES) was analyzed. The recruitment was performed using computer-assisted-telephone-interviewing and one-day 24-h dietary recall retrieved from all participants. The updated, second version, of FoodEx2 food classification system was applied to extract data on food group consumption. The participation rate was $53 \% ; 6.2 \%$ of the participants declared to follow a special diet, the most prevalent being hypo-caloric diets (55.7\% of special diets). Men compared to women presented significantly higher intakes of "grains and grain-based products", "meat and meat products", "animal and vegetable fats and oils and primary derivatives" and "alcoholic beverages" ( $\mathrm{P}$ for all $<0.001$ ); moreover, men had lower intakes of "milk and dairy products", "water and water-based beverages" and "products for non-standard diets, food imitates and food supplements" (P for all $<0.001$ ). Differences in food group intake among age groups, geographical regions and educational level groups were also identified ( $P$ for all $<0.05$ ).

Conclusions: Data on the consumption of more than 70 food groups and sub-groups were illustrated in different strata. The present analysis could be considered as an updated source of information for future nutrition research in Italy and in the EU.

(c) 2017 The Italian Society of Diabetology, the Italian Society for the Study of Atherosclerosis, the Italian Society of Human Nutrition, and the Department of Clinical Medicine and Surgery, Federico II University. Published by Elsevier B.V. All rights reserved.
\end{abstract}

* Corresponding author. Laboratory of Molecular and Nutritional Epidemioloy, Department of Epidemiology and Prevention, IRCCS Istituto Neurologico Mediterraneo Neuromed, Via dell'Elettronica, 86077 Pozzilli (IS), Italy.

E-mail address: licia.iacoviello@neuromed.it (L. Iacoviello).

${ }^{1}$ INHES Investigators are listed in the Appendix.

\section{Introduction}

Dietary habits evolve over time, being influenced by many factors and complex interactions. Income, prices, individual preferences and beliefs, cultural traditions, as well as geographical, environmental, social and economic factors all interact in a complex manner to shape dietary consumption patterns. This indicates a specific need for the study of dietary habits of the populations. 
The traditional Mediterranean diet (MeD), an eating behavior model, with recognized health benefits in primary and secondary prevention of chronic diseases [1-4] is less followed by southern European populations in the last decades [5-7]. This occurs also in Italy and requires an updated monitoring in public health strategies.

The last suitable Italian public health surveillance program on nutrition has been held by the INRAN (National Institute for Research on Foods and Nutrition) covering the years 2005-06 [8,9]. Since then, no further information has been gathered at the national level for food intake all over Italy.

Furthermore, a serious challenge in modern nutritional epidemiology is the harmonization of the methods used for the extraction of dietary data across different populations. This will allow a better understanding of differences among samples, also in association with health outcomes and disease progression.

A major part in the harmonization of dietary assessment methodologies consists in the use of a common food classification system. Recent efforts have been carried out by the European Food Safety Authority (EFSA) to establish a largely appreciated food classification system, presented with a newly updated version of FoodEx2 [10]. To our knowledge, there is presently no large epidemiological study that has published food group consumption data elaborating such last version of FoodEx2.

Thus, this study aims at evaluating and presenting the most recently updated piece of information on the dietary habits of Italians, especially food group consumption, using data derived from the Italian Nutrition \& HEalth Survey (INHES). The use of an updated, second version, of FoodEx2 food classification system adds to the novelty and originality of the present study.

\section{Methods}

\section{Study population}

The INHES study is a telephone-based survey on nutrition and health specifically designed to collect information on dietary habits (quality, quantity and patterns), food choice determinants, and food health awareness of the Italian population according to geographical distribution, age, gender and socio-economic profile. Between November 2010 and November 2013, 9319 women and men aged $\geq 5$ years from all over Italy were enrolled.

Firstly, subjects $(\mathrm{n}=9106)$ in the age range 35-79 years, recruited in the 2008-2012 wave of the Italian Cardiovascular Epidemiologic Observatory (participation rate $53 \%$, from $40 \%$ to $85 \%$ in the different regions) [11,12] were invited to participate in the INHES survey. Once they accepted, participants were asked to invite one relative older than 79 or younger than 35 years to join the survey. Finally 5385 (59.1\%) from the original population and 3754 from their relatives were included in the survey.

The sampled subjects were distributed in the four seasons (excluding Christmas, Easter and mid-August periods). The survey calendar was organized to capture an adequate proportion of weekdays and weekend days at group level.
The recruitment was performed using computerassisted-telephone-interviewing (CATI): one-day 24h dietary recall [9], the Italian version of the European Food Propensity Questionnaire [13], questionnaire of nutrition related behavior, anamnestic questionnaire on health status, risk factors and anthropometry and SF12 questionnaire on health perception [14] were administered.

For the purpose of the present study the adult (18-96 years) population with completed dietary information more than 50\% (20 participants eliminated) of 8944 ( 4768 women and 4176 men) was analyzed. The INHES study was approved by the Ethical Committee of the Catholic University of Rome.

\section{Dietary assessment}

Each participant received by mail a short photographs atlas and guidance notes to estimate portion sizes (with instructions to quantify the portions used by children) developed on the basis of EPIC-SOFT picture book [15] and a hardcopy diary structured by meal, where all the information on food consumption the day before the telephone interviews was self-recorded. All foods and drinks consumed (including tap and bottled water), both at and outside home, were recorded. The day after, participants were interviewed by telephone by trained and standardized interviewers, starting from the self-recorded diary, by using a computer based 24-h dietary recall interview (24-HDR) software.

For every eating occasion, subjects were asked to carefully record and recall: time, place of consumption, detailed description of foods (or beverages), quantity consumed and brand (for manufactured foods). Portion sizes were reported by subjects with the help of a picture booklet. Moreover, it was asked if they were following a particular diet and if the consumption they had reported differed from their usual consumption.

The data management system INRAN-DIARIO 3.1 developed by INRAN $[9,16]$ in previous surveys $[9,17]$ was used for data coding, data entry and data processing. This software includes several checkpoints to ensure the accuracy and completeness of the data recorded and allows each interviewer to create new temporary food codes for all the food items and recipes that are not present in the databanks. Four databases were used to transform the data reported by subjects into the weight of single foods. The portions estimated by subjects with the help of the picture booklet are linked to the specific weight of each food item. Any missing food consumed during the survey was added to the food composition database.

\section{Food group classification by applying the FoodEx2 system}

All participants food consumption of single food items or recipes was "translated" by the nutrition specialist during the interview into food items or recipes included in the food list of the data management system INRAN-DIARIO 3.1. The current food list includes information for almost 2000 single food items and 1700 recipes typically consumed in Italy. All food recipes consumption was automatically divided into food items intake by the software itself. The final output-database 
included information for the daily consumption of the 2000 single food items that were included in the software food list.

The classification of single food items to food groups was performed using the Exposure Hierarchy tree of the European Food Safety Authority's (EFSA's) FoodEx2 (version 2) classification system $[10,18]$, recently available (April 2015). Two levels of food hierarchy were used and are described in the present work as "food groups" and "sub-groups".

Supplemental Table 1, illustrates the generated food groups and sub-groups for INHES dietary data including a description and the number of food items classified in each occasion.

Out of the 133 "food" and "sub-group" of the first two levels of hierarchy tree of FoodEx2 food classification system the 74 were finally elaborated (19 food groups and 55 sub-groups). Within the remaining food groups and subgroups that are not reported in the present analysis, none of the food items of INRAN-DIARIO 3.1 were categorized or the consumption was almost zero. Also the food group "Food products for young population" was not reported in this work since we are referring to the adult population.

\section{Qualitative food frequency consumption assessment}

Beside the quantitative assessment and analysis of food group intake through the 24-h dietary recall interview (24-HDR), all participants also reported qualitative data on food frequency consumption by the elaboration of a food propensity questionnaire validated in previous studies $[13,19,20]$. The frequency of consumption of cooked vegetables, raw vegetables, fruits, cheese, legumes, rice, pasta, red meat, white meat, fish and milk was reported and presented as follows: $0-3$ times/month, 1-2 times/week, 3-4 times/week, 5-6 times/week, $\geq 1$ time/day.

\section{Other factors}

Body weight and height were self-reported and BMI (kg/ $\mathrm{m}^{2}$ ) was calculated. Since no anthropometric measurement was performed in INHES studies, these parameters are under the related self-reporting bias.

\section{Statistical analysis}

Descriptive results on food group and sub-group consumption were presented as mean, median, standard deviation, 95th and 99th percentiles in total population and in consumers separately. The analyses were also stratified by gender, age group (i.e. 18-65 years and >65 years), geographical region (i.e. North, Central and South Italy) and educational level group (i.e. elementary or secondary school, high school and university or college degree).

Normality of continuous dietary data was tested using the Shapiro-Wilk's test [21]. The results indicated that all distributions were skewed ( $P$ from Shapiro-Wilk's test for all $<0.001)$. This finding was also confirmed graphically. Since the data distributions were skewed, non-parametric tests were elaborated. In fact, differences in food group consumption between gender and age groups were assessed with Mann-Whitney's test and between geographical region and educational level groups using Kruskal-Wallis's test. Bonferroni correction for multiple comparisons (18 tests and significance level $\alpha=0.05$ ) yielded a corrected $\alpha=0.002$, which indicated significant differences at the aforementioned analysis.

Categorical variables were presented as frequencies and their associations were tested using Pearson's $\mathrm{X}^{2}$-test. The assessment of differences between age groups for height, weight, BMI and energy intake was performed using Student's t-test since data were normally distributed.

For all food groups separately, the percentage of consumption during four meal occasions (i.e. breakfast, lunch, dinner and snack) out of the total daily consumption of each food group was calculated and presented in Figures.

Two-sided P-value $<0.05$ was considered as statistically significant. STATA version 9 software was used for all calculations (STATA Corp., College Station, TX, USA).

\section{Results}

Among survey days, $89.8 \%$ were weekdays (i.e. Monday to Friday). The seasonal distribution of survey days was as

Table 1 Characteristics of INHES participants according to gender and age group.

\begin{tabular}{|c|c|c|c|c|c|c|}
\hline & \multicolumn{2}{|c|}{ Women $(\mathrm{N}=4768)$} & \multirow[t]{2}{*}{ P-value ${ }^{b}$} & \multicolumn{2}{|c|}{$\operatorname{Men}(\mathrm{N}=4176)$} & \multirow[t]{2}{*}{ P-value } \\
\hline & $\begin{array}{l}18-65 \text { years } \\
(\mathrm{n}=3295)\end{array}$ & $\begin{array}{l}>65 \text { years } \\
(\mathrm{n}=1473)\end{array}$ & & $\begin{array}{l}18-65 \text { years } \\
(\mathrm{n}=2775)\end{array}$ & $\begin{array}{l}>65 \text { years } \\
(n=1401)\end{array}$ & \\
\hline Educational level (\%) & & & $<0.001$ & & & $<0.001$ \\
\hline$\leq 8$ years & 33.7 & 71.5 & & 33.5 & 64.8 & \\
\hline$\overline{9}-13$ years & 44.8 & 22.3 & & 48.1 & 28.7 & \\
\hline$\geq 14$ years & 21.3 & 6.2 & & 18.4 & 8.6 & \\
\hline Physical activity level (\%) & & & $<0.001$ & & & $<0.001$ \\
\hline Non-physically active & 79.0 & 90.2 & & 74.1 & 90.2 & \\
\hline Physically active & 21.0 & 9.8 & & 25.9 & 9.8 & \\
\hline Height $(\mathrm{cm})$ & $163(6)$ & $160(6)$ & $<0.001$ & $175(7)$ & $170(7)$ & $<0.001$ \\
\hline Weight (kg) & $64.5(12.3)$ & $66.7(12.0)$ & $<0.001$ & $80.4(13.4)$ & $78.1(11.6)$ & $<0.001$ \\
\hline $\operatorname{BMI}\left(\mathrm{kg} / \mathrm{m}^{2}\right)$ & $24.4(4.6)$ & $26.0(4.4)$ & $<0.001$ & $26.3(6.1)$ & $26.9(6.0)$ & 0.001 \\
\hline Energy intake (kcal/day) & $1834(686)$ & $1700(539)$ & $<0.001$ & $2129(779)$ & $1998(628)$ & $<0.001$ \\
\hline
\end{tabular}




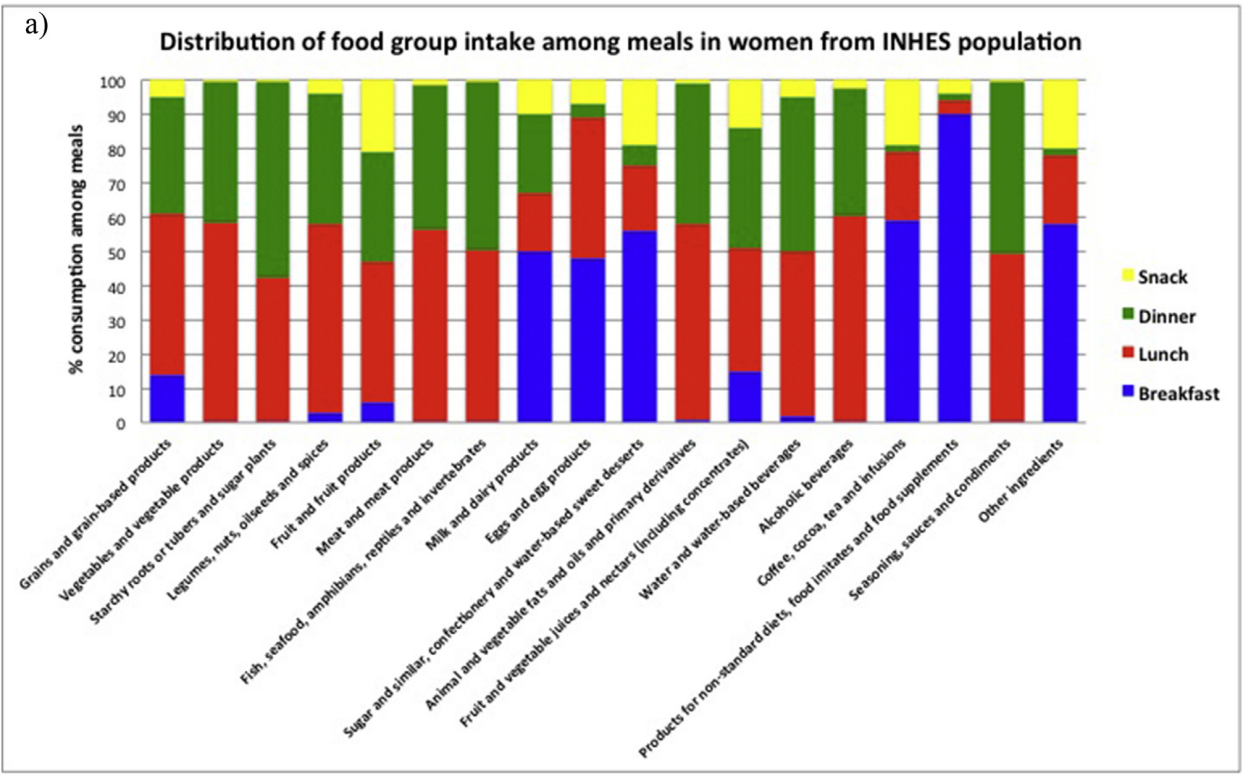

b)

Distribution of food group intake among meals in men from INHES population

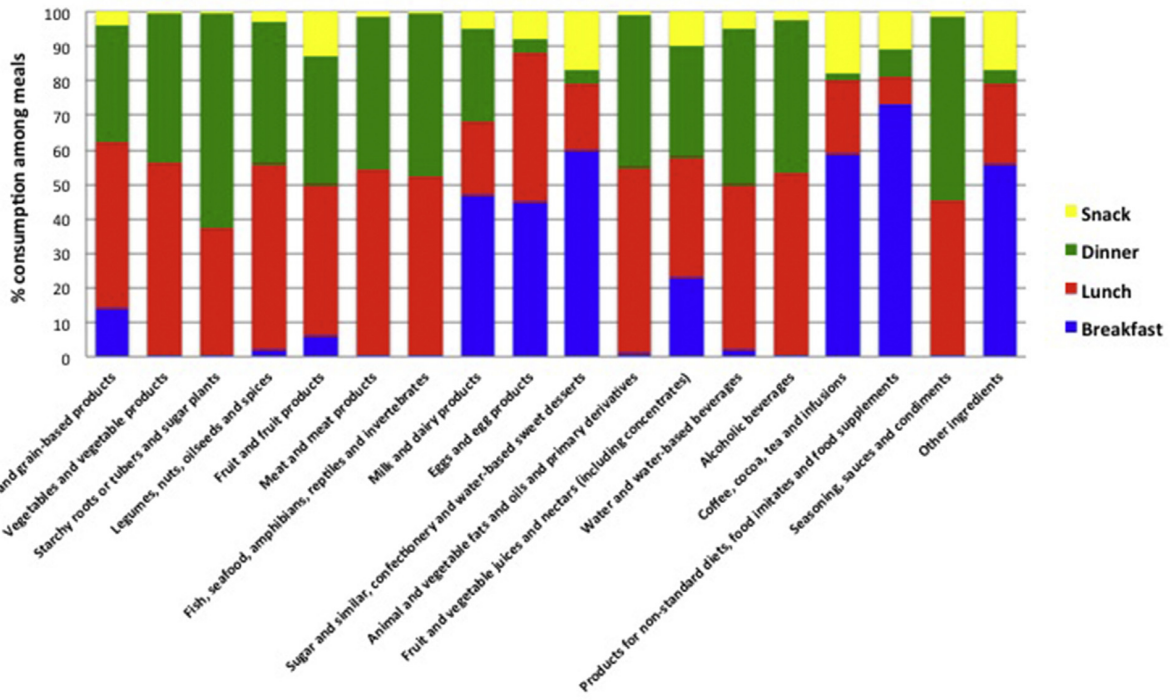

Figure 1 a: The distribution of food group consumption among meals in women, expressed as percentages of total consumption for each food group in the 4 meals considered (breakfast, lunch, dinner and snack). b: The distribution of food group consumption among meals in men, expressed as percentages of total consumption for each food group in the 4 meals considered (breakfast, lunch, dinner and snack). 


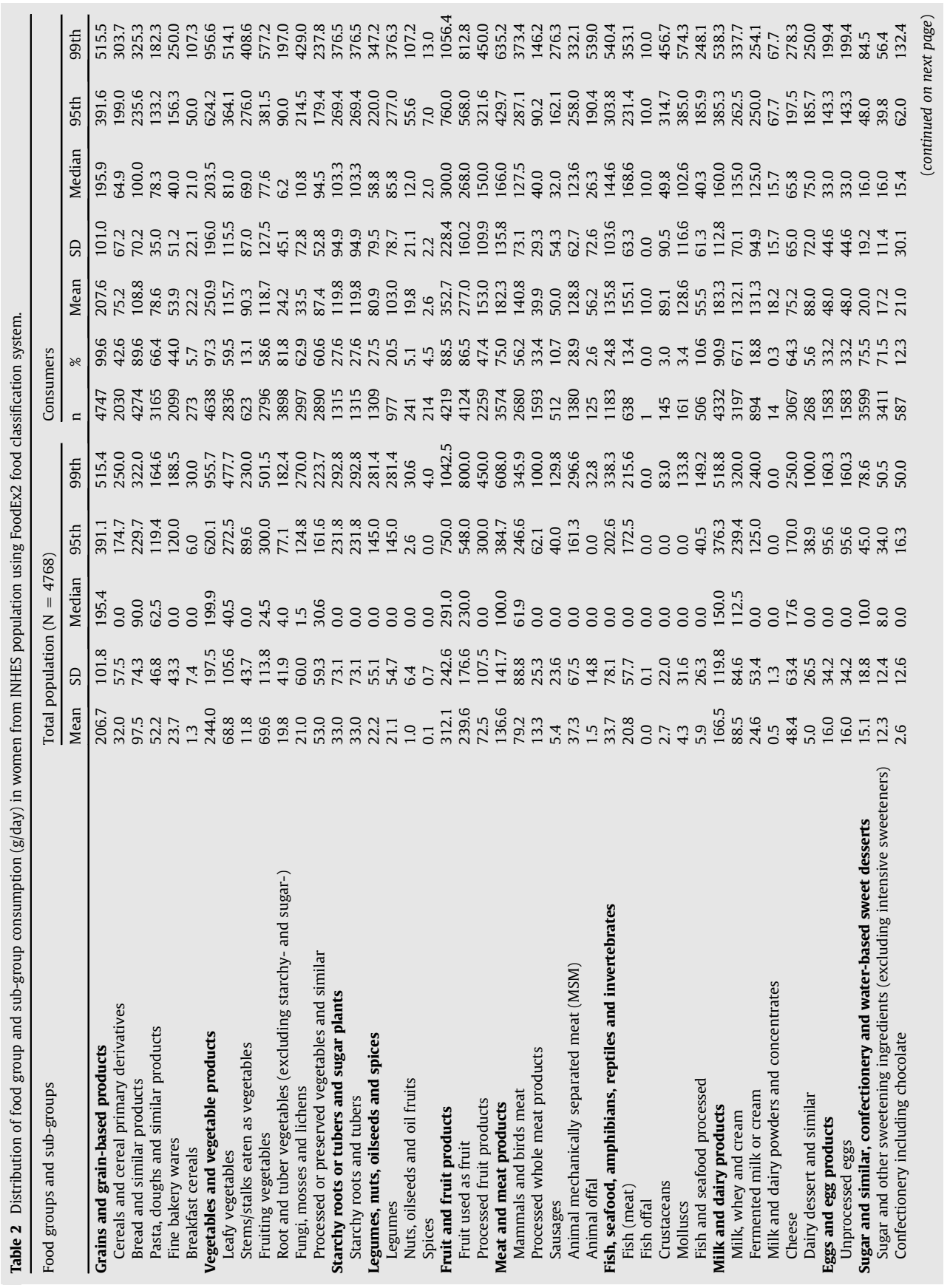




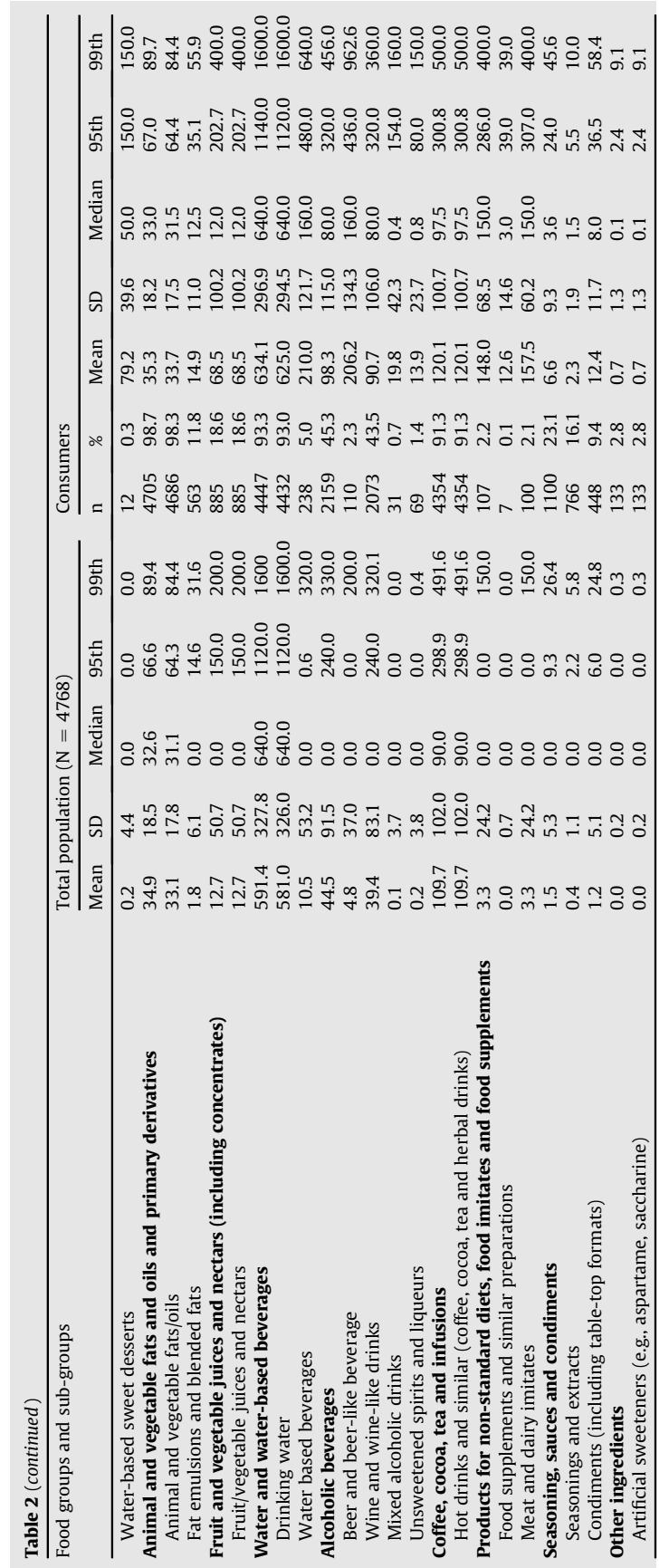




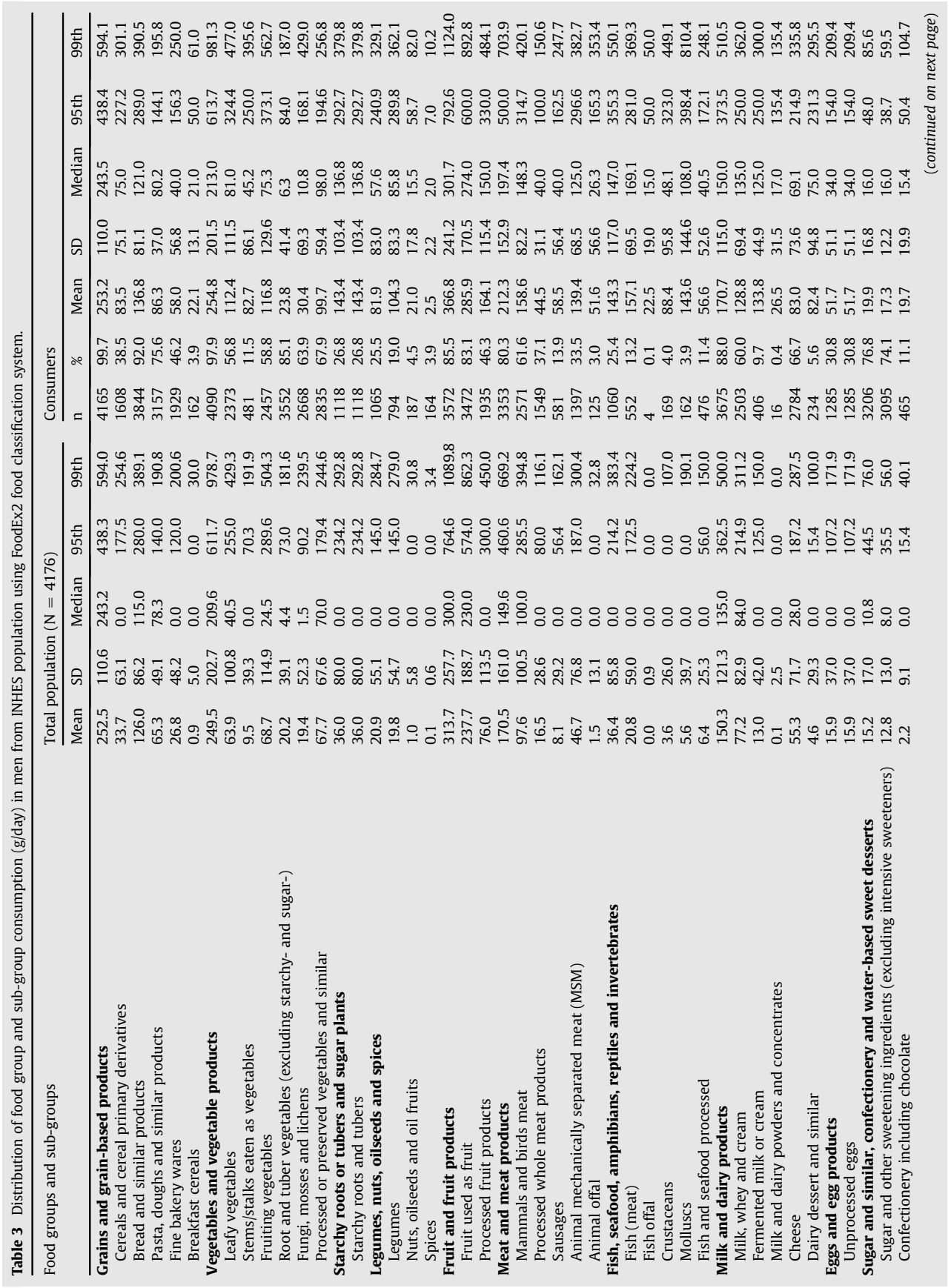




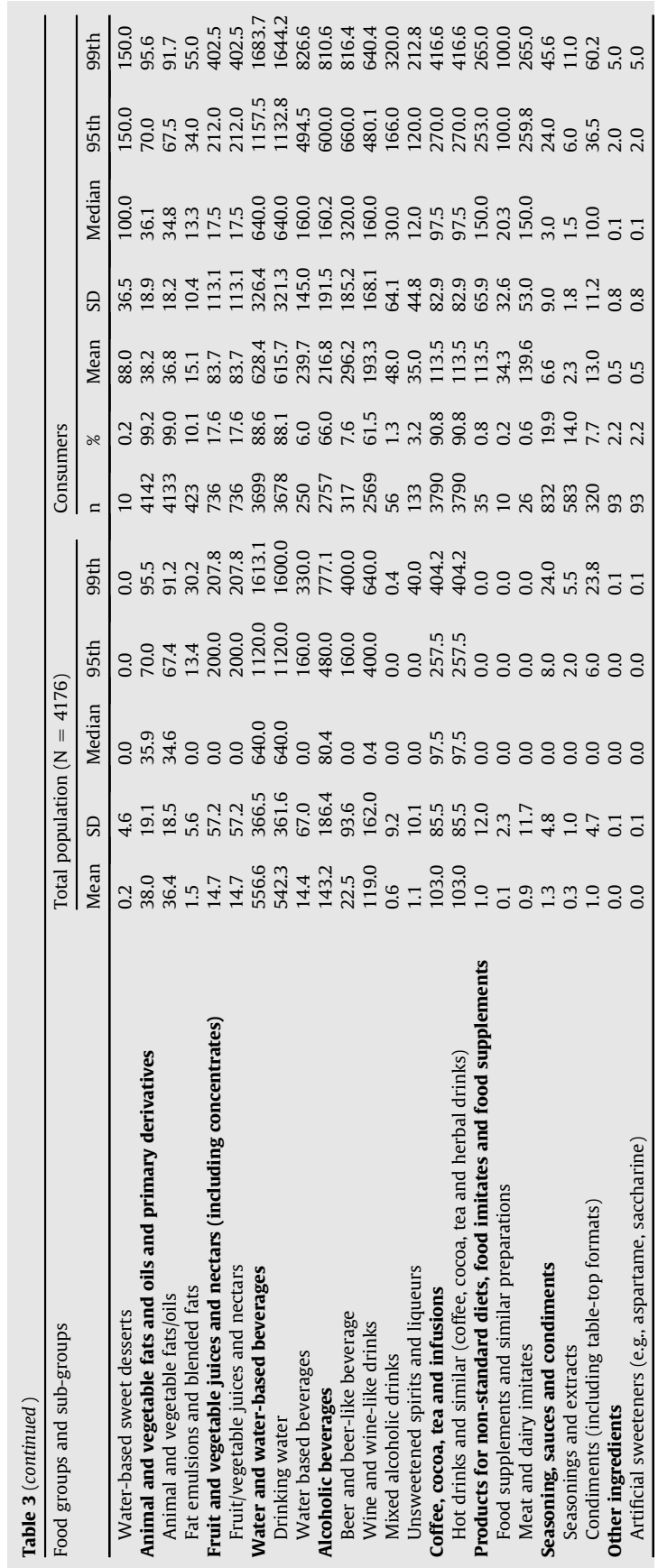




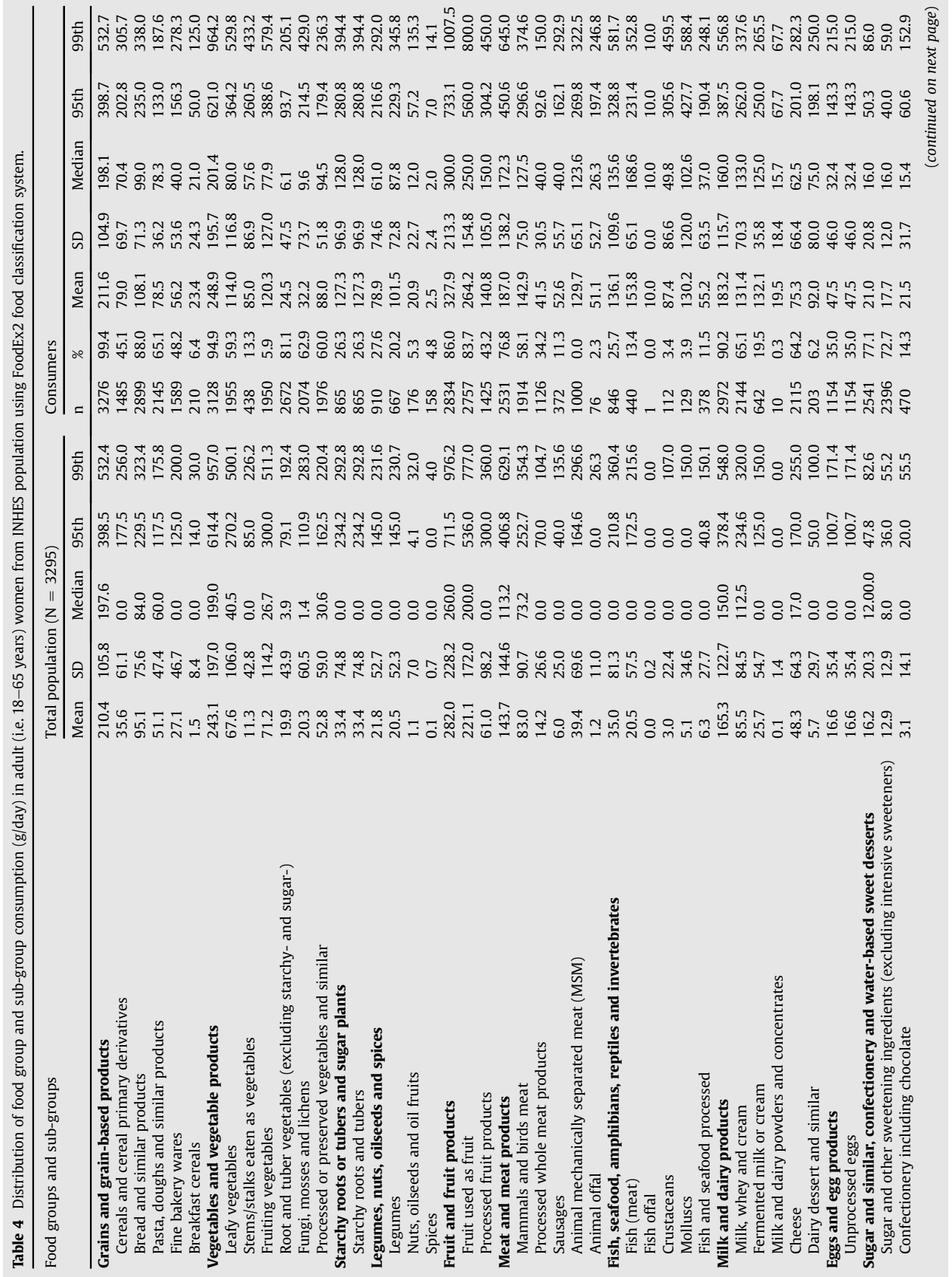




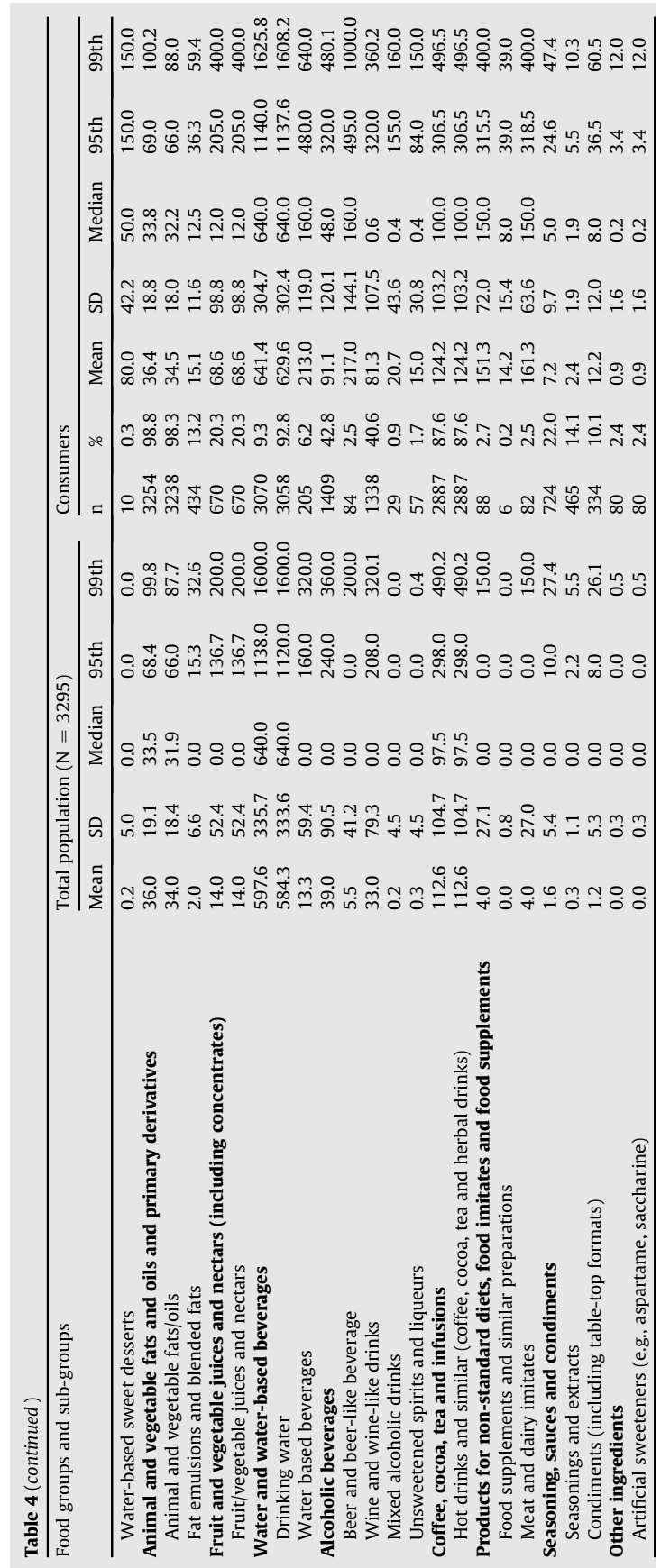




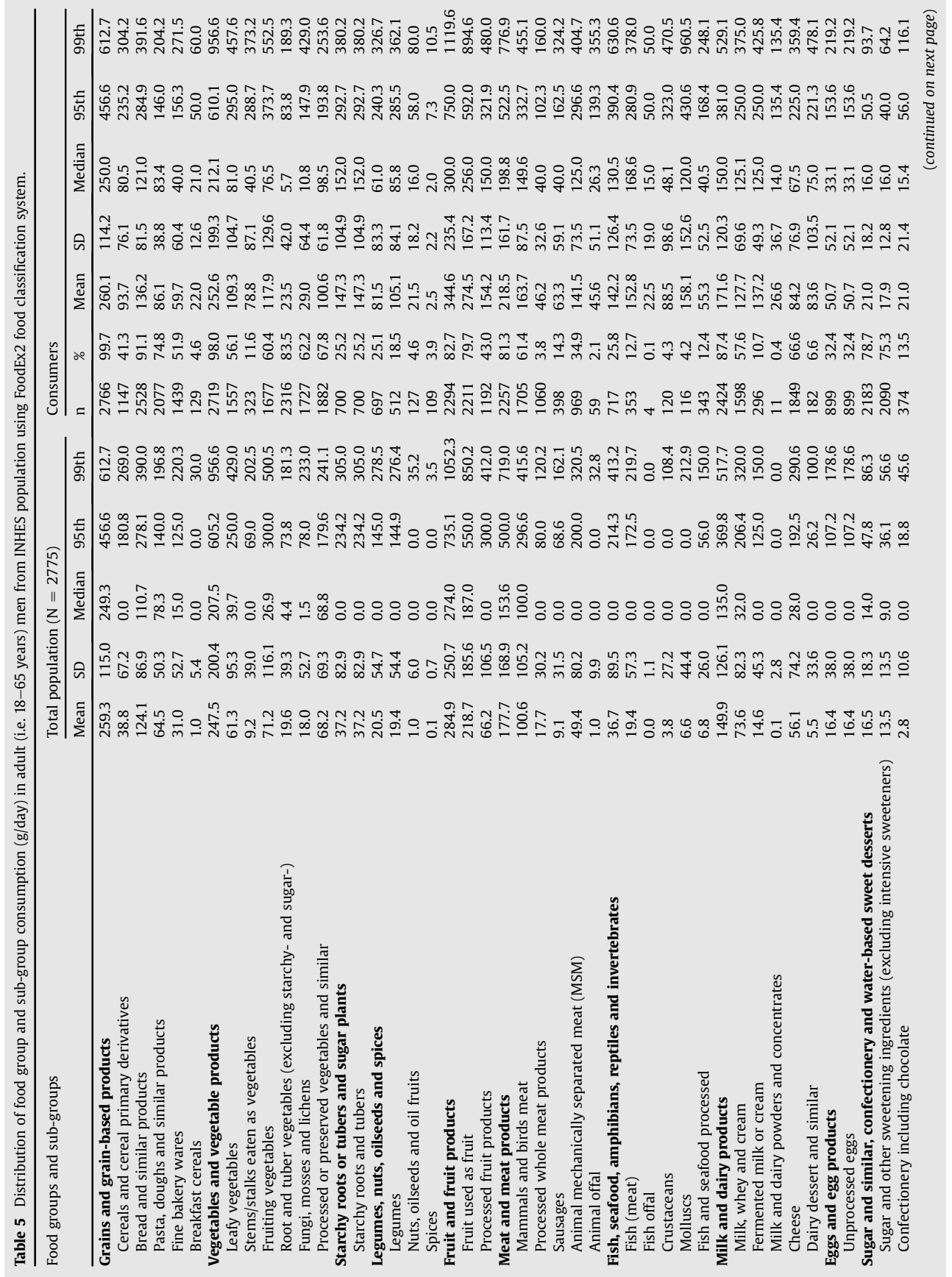




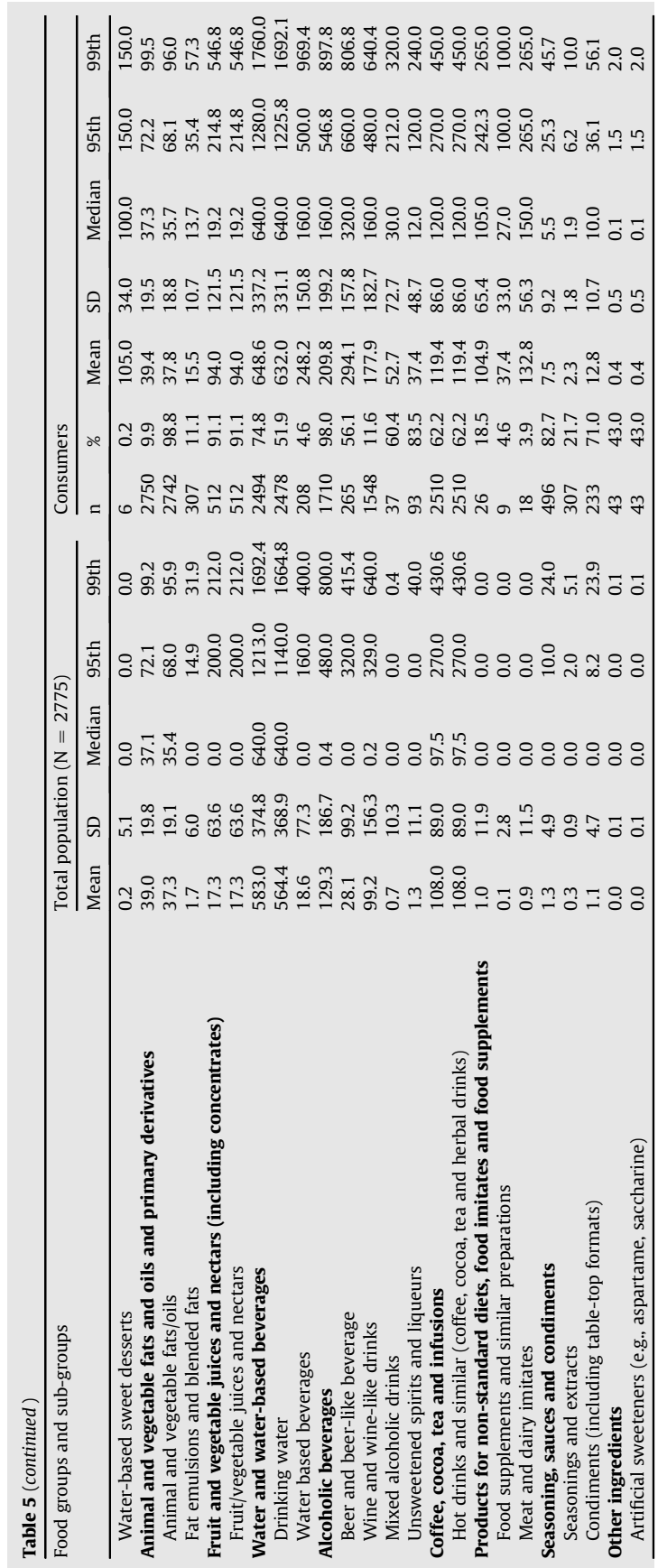




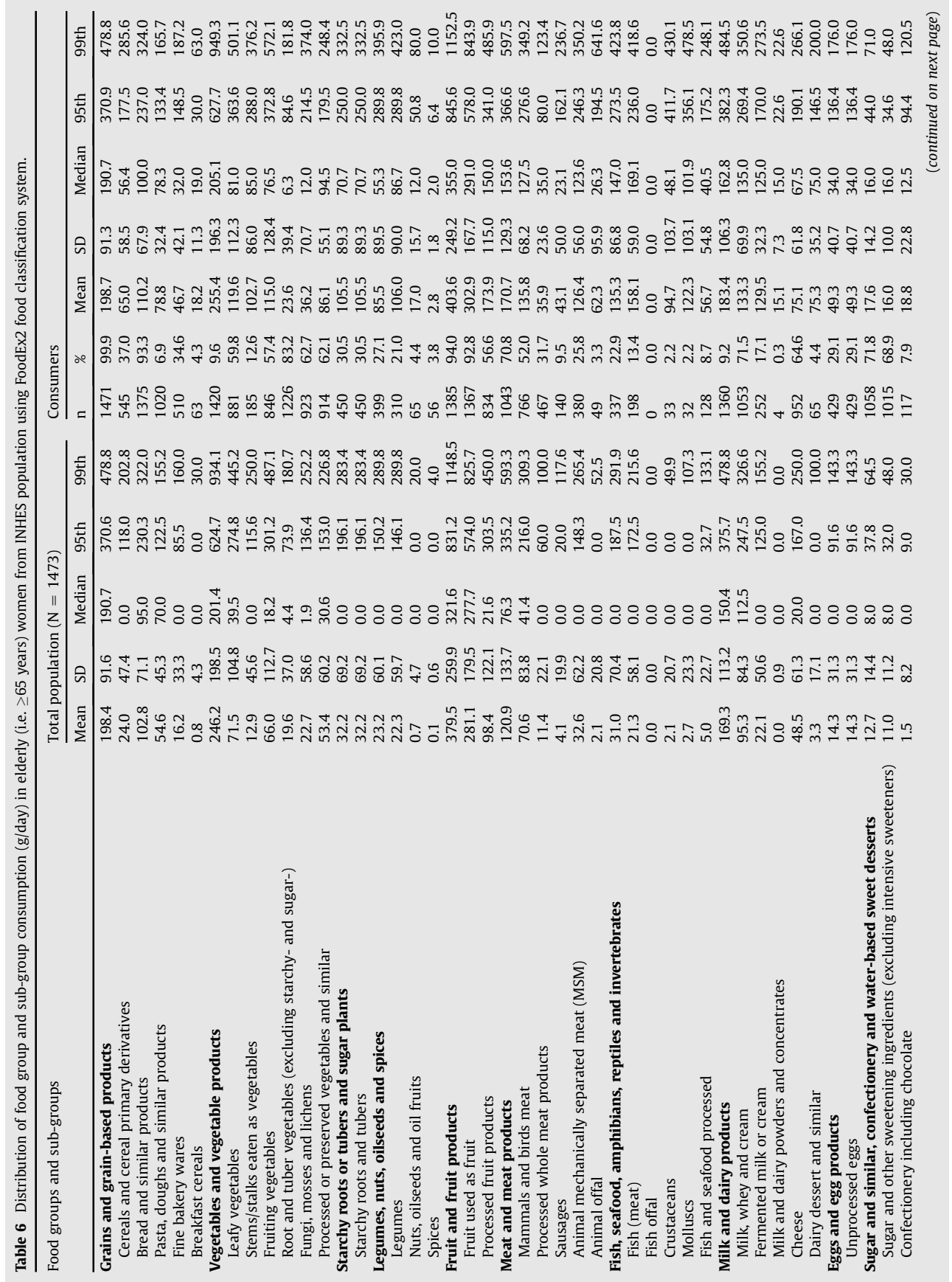




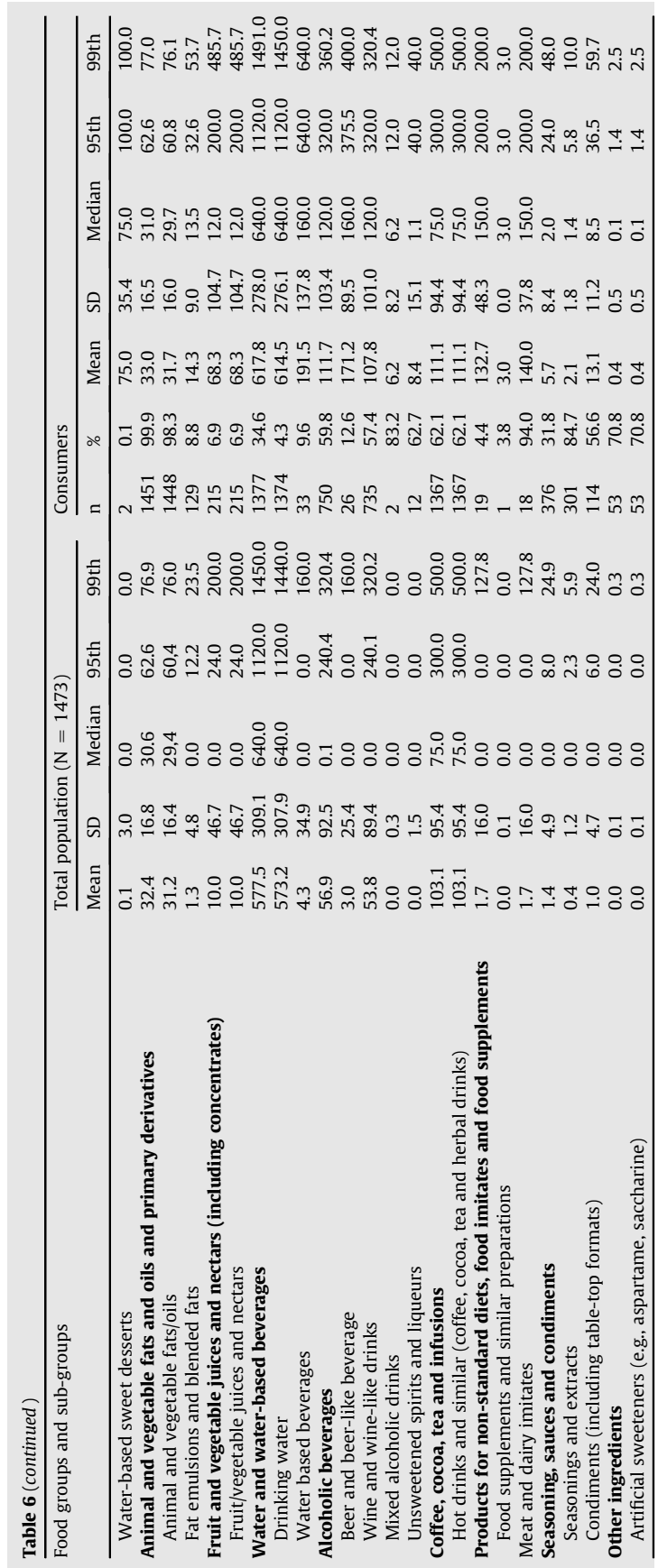




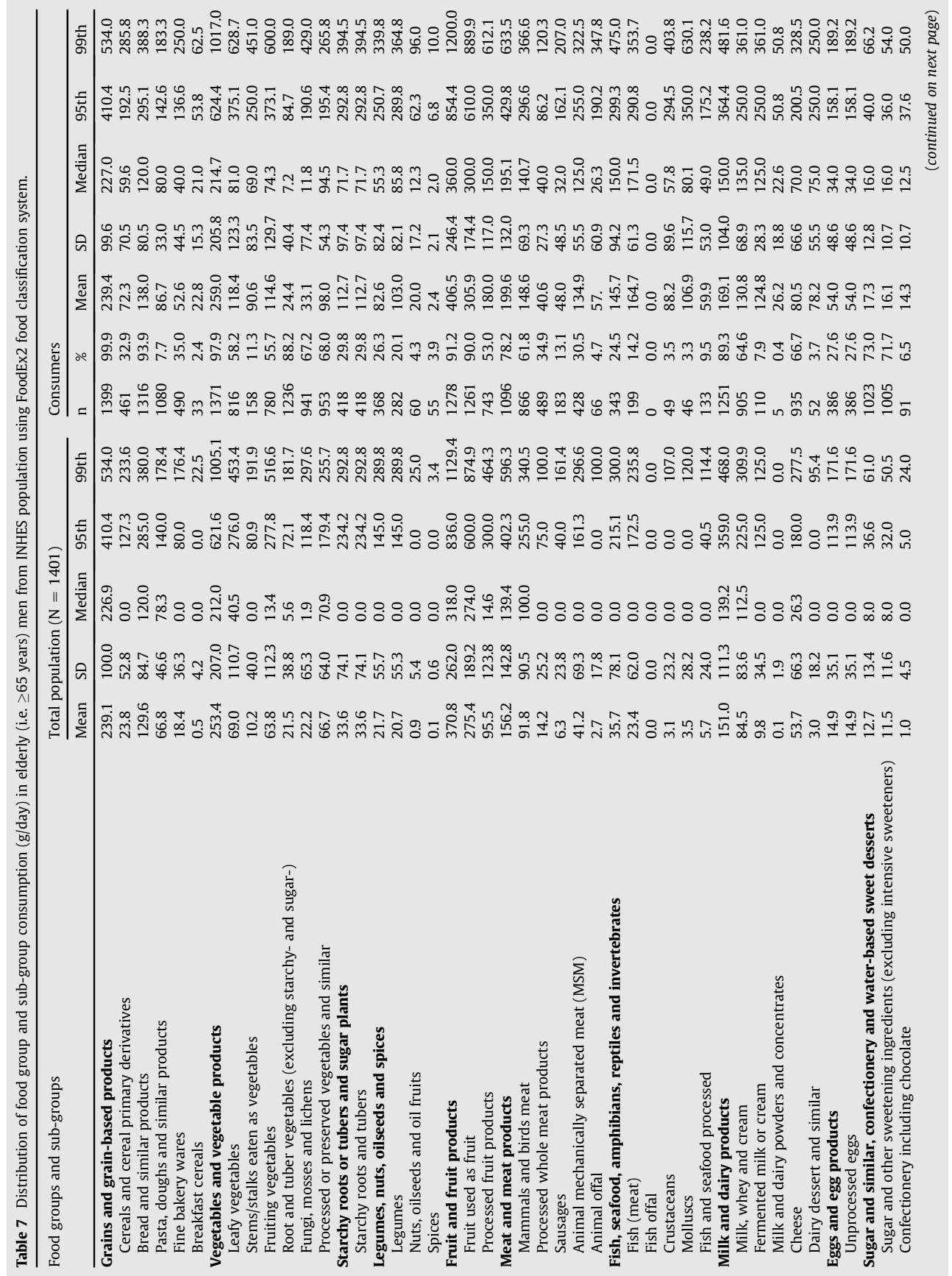




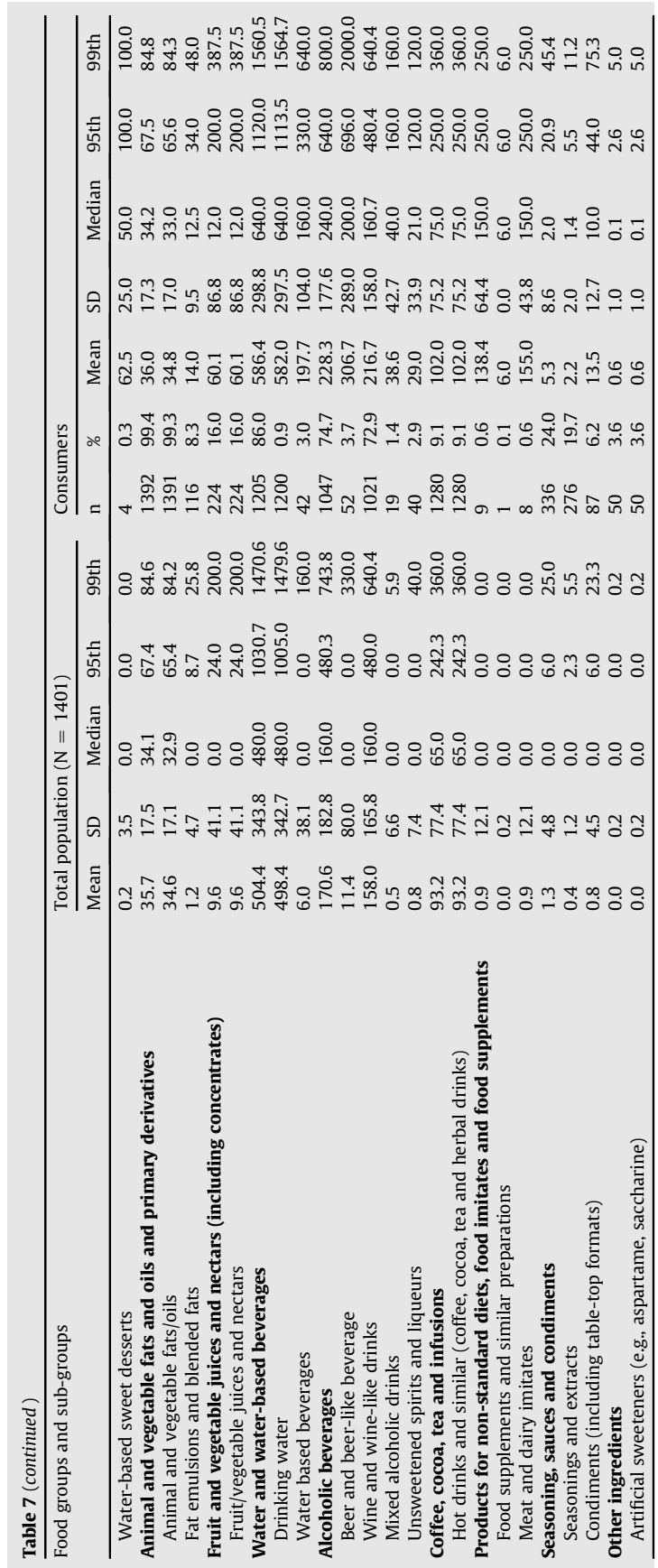


follows: $30.7 \%$ in autumn, $23.5 \%$ in winter, $25.1 \%$ in spring and $20.7 \%$ in summer.

The participants' general characteristics according to gender and age group are presented in Table 1. In both genders the elderly population seemed to be less educated and physically active with higher BMI compared to the younger population ( $P$ for all $<0.05$ ). Both women and men aged 18-65 years reported higher total energy intake than the elderly population ( $\mathrm{P}$ for both $<0.001$ ).

As many as $6.2 \%(\mathrm{n}=555)$ of the INHES participants declared that they were following a special diet. Hypocaloric diets used for the reduction of body weight represented $55.7 \%(n=309)$, while diets for the primary or secondary prevention of diseases (i.e. diabetes, hypertension) were also followed.

In addition, $7.8 \%(n=702)$ of the participants reported that their food consumption the day prior to the interview-day was different from usual (i.e. higher or lower) due to different reasons (i.e. special dinner with guests, celebrations, reduced food intake due to clinical tests etc.).

Regarding meal consumption, 97.6\%, 99.1\%, 99.0 and $30 \%$ of the INHES population reported having breakfast, lunch, dinner and snack during the day, respectively. The most frequent meal time occasion was 7:00-7:30am for breakfast (39.5\%), before 1:00pm for lunch (63.8\%) and 8:00-8:30pm for dinner (51.9\%), while snacking time varied.

The distribution of food group consumption among meals in women and men is illustrated in Fig. 1a and b, respectively. In both genders "vegetable and vegetable products", "starchy roots or tubers and sugar plants", "fish, seafood, amphibians, reptiles and invertebrates" and "seasoning, sauces and condiments" were only consumed during lunch and dinner. In addition, "legumes, nuts, oil seeds and spices", "meat and meat products", "animal and vegetable fats and oils and primary derivatives", "water and water based beverages" and "alcoholic beverages" were mainly consumed over lunch and dinner.

"Grains and grain-based products", "fruit and fruit products" and "fruit and vegetable juices and nectars" were mostly consumed over lunch and dinner but sometimes also at breakfast or as snack choices.

On the contrary, "milk and dairy products", "eggs and egg products", "sugar and similar, confectionery and water based sweet dessert", "coffee, cocoa and tea and infusions", "products for non-standard diets, food imitates and food supplements and artificial sweeteners" were mainly consumed over breakfast, less for lunch and dinner and sometimes as snack choice.
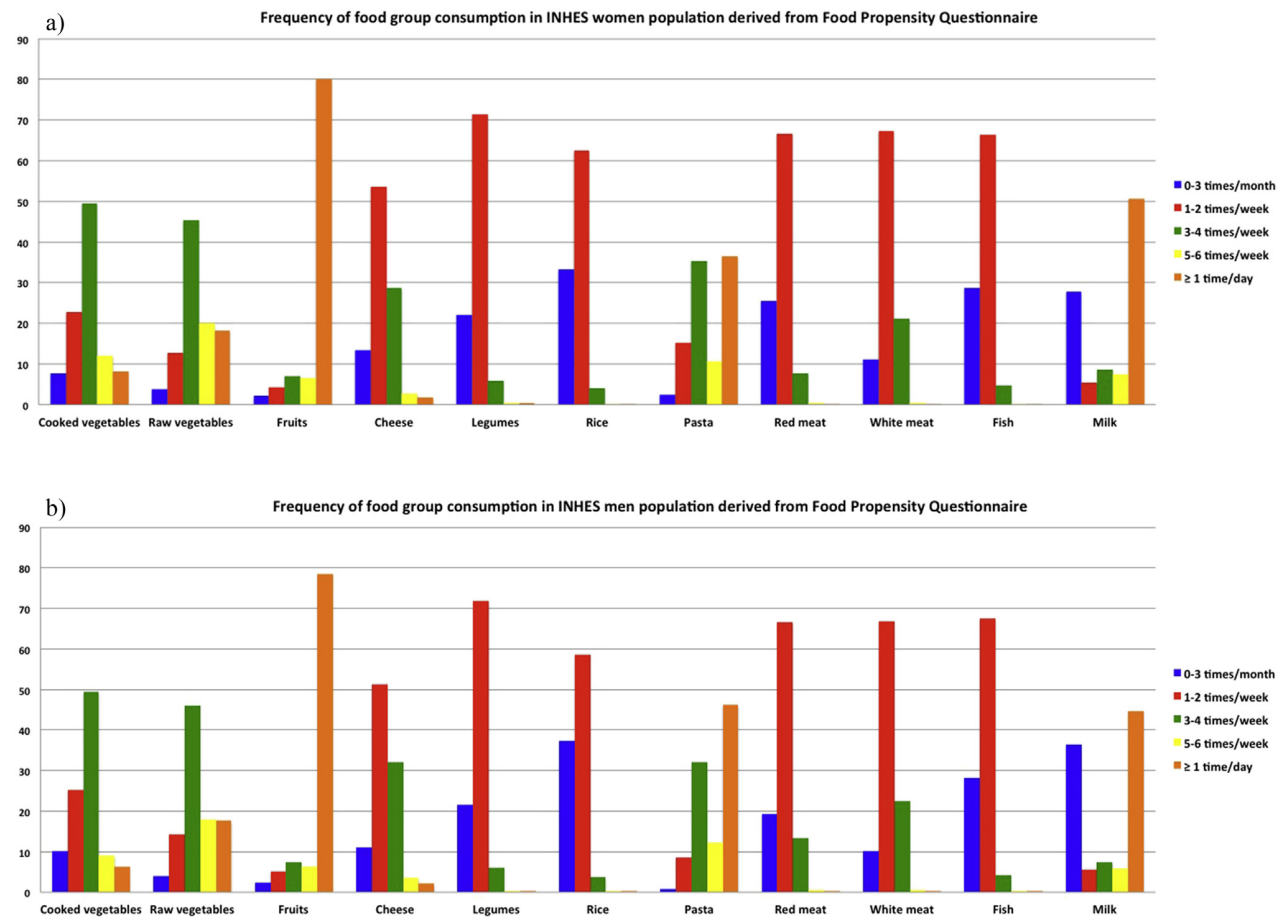

Figure 2 a: The frequency of food group consumption using data from the food propensity questionnaire in the women population. b: The frequency of food group consumption using data from the food propensity questionnaire in the men population. 
Detailed descriptive results on food group and subgroup consumption stratified by gender and age group are presented in Tables $2-7$. In addition, similar descriptive analyses stratified by gender, geographical origin and education level are included in Supplemental Tables 2-13.

The highest number of consumers was observed for "grains and grain-based products", "vegetable and vegetable products" and "animal and vegetable fats and oils and primary derivatives" in both genders.

Supplemental Table 14 presents the P-values derived from the comparisons in food group intake between genders, age groups, geographical regions and education level groups.

Men compared to women presented statistically significant higher intakes of "grains and grain-based products", "meat and meat products", "animal and vegetable fats and oils and primary derivatives" and "alcoholic beverages" ( $P$ for all $<0.001<0.002=$ Bonferroni corrected $\mathrm{p}$-value for 18 comparisons), (Tables 2 and 3 and Supplemental Table 14 ); men had also lower intakes of "milk and dairy products", "water and water-based beverages", and "products for non-standard diets, food imitates and food supplements" ( $\mathrm{P}$ for all $<0.001<0.002=$ Bonferroni corrected $\mathrm{p}$-value for 18 comparisons), (Tables 2 and 3 and Supplemental Table 14).

The analysis of differences between age groups (i.e. 18-65 years, >65 years) in both men and women indicated significantly higher consumption of different food groups in adults compared to the elderly ( $P$ for all $<0.001<0.002=$ Bonferroni corrected $\mathrm{p}$-value for 18 comparisons), (Tables 4-7, and Supplemental Table 14).

Furthermore, statistically significant geographical differences in food group consumption were observed in both genders (Supplemental Tables 2-7 and 14). Interestingly, both women and men from Southern Italy reported greater consumption of "grains and grain-based products" and "meat and meat products" (P for all $<0.001<0.002=$ Bonferroni corrected p-value for 18 comparisons); while North sample population had greater intake of "coffee, cocoa, tea and infusions" (P for all $<0.001<0.002=$ Bonferroni corrected $\mathrm{p}$ value for 18 comparisons).

In addition, food group consumption differed among educational level groups (Supplemental Tables 8-13 and 14). Notably, participants (both women and men) from the highest educational level group had higher intake of "animal and vegetable fats and oils and primary derivatives", "fruit and vegetable juices and nectars", "coffee, cocoa, tea and infusions" and "products for nonstandard diets, food imitates and food supplements" ( $\mathrm{P}$ for all $<0.001<0.002=$ Bonferroni corrected $\mathrm{p}$-value for 18 comparisons).

\section{Qualitative results on food frequency consumption}

Figure $2 \mathrm{a}$ and $\mathrm{b}$, illustrate the frequency of food group consumption using data that were derived through the food propensity questionnaire in women and men INHES participants, correspondingly.
Raw vegetables, fruits, pasta and milk were among the most frequently consumed food groups on a daily basis (i.e. $\geq 1$ time/day) in both genders. As many as $18.3 \%$ and $17.6 \%$ of women and men respectively reported to consume raw vegetables $\geq 1$ time/day while the same percentage for fruits were $80.1 \%$ and $78.6 \%$ ( $P$ for sex differences for both $>0.05$ ).

\section{Discussion}

\section{The INHES project}

Despite the importance of monitoring dietary habits in public health perspectives, according to the best of our knowledge, there has been no study in the last decade evaluating at an epidemiological level the dietary patterns in Italy. Our findings illustrate a comprehensive descriptive analysis of food group consumption using recent data coming from all over Italy. Results by gender, age group, geographical region and educational level group or type of meal were generated to provide multi-dimensionality in our approach. The elaboration of accurate dietary assessment methodologies and the newly updated EFSA's FoodEx2 food classification system [10] add to the novelty of the present work.

The last effort for an accurate evaluation of dietary habits at a national level in Italy was performed in 2005-06, when INRAN retrieved data from 1329 households corresponding to 3323 women and men [9]. Food group consumption data were collected at individual level in 3 consecutive days using hard-copy diaries structured by meal and the data management system INRAN-DIARIO 3.1. The researchers published in 2008 a descriptive analysis of food group intake, presenting results by gender and age group after the elaboration of a self-developed food classification system [9].

Other nationwide Italian food consumption surveys have been carried out. The first approach was performed at household level and was held by INRAN in the early 80's [22]. In the 90's, the EPIC (European Prospective Investigation into Cancer and Nutrition) study collected and presented data from 1990 to 1995 based on single 24-HDR from different regions in Italy [23]. Almost at the same time period, the INN-CA Study group (1994-1996) published nationwide descriptive results on food consumption derived from food data forms (diaries and inventories) on 1147 households, corresponding to 2734 male and female individuals [24].

On the contrary, our data have been collected until very recently (2012) and it could be considered, at the moment, as the most updated study at a national Italian level. In addition, our dietary assessment methodology consisted mainly in 24-HDR interviews assisted by the data management system INRAN-DAIRIO 3.1 and it is relevant and comparable to those used in the most recent study of INRAN on 2005-06 [9].

Moreover, a significant point of originality of the INHES project and strength of this work was the larger sample 
size compared to previous studies [9], covering the majority of geographical and socio-economical strata.

Furthermore, to the best of our knowledge, this is the first study at European level that elaborated EFSA's FoodEx2 classification system, recently available in April 2015 [10]. This is another strength that applies to the modern meta-analytic needs in epidemiological studies. In fact, the use of a common food classification system among studies in different EU or other populations contributes to the harmonization of the dietary data and makes them better comparable for meta-analysis.

Despite the serious importance of its application, the categorization of food items into food groups or subgroups of FoodEx2 was still challenging. On the one hand, the EFSA's classification system includes several levels of hierarchy and various tools to assist the categorization of foods items into groups such as facet descriptors. On the other hand, main aim of the application of a food classification system is to merge the epidemiological information into food groups that later on can be easily applied to statistical or other tests and models. Keeping a balance between the analytic perception of FoodEx2 and the needs of summarizing information into food groups in epidemiological analysis is of high importance for current and future research in the field.

The elasticity that FoodEx2 provides to the epidemiological researcher in choosing different levels of hierarchy and various tools to do so, proved to be very useful in its application to real data. In the present analysis, the first two levels of hierarchy of FoodEx2 were applied providing information on a large range of 74 food groups and subgroups. In addition, it is still possible to use a more detailed or specific approach in food categorization and presentation of data, by using various facets descriptors or levels of hierarchy, according to the aim of the study (e.g. the low or whole fat content in milk and dairies that is not described in the first two levels of FoodEx2 hierarchy requires the analysis of these data in other hierarchy levels).

\section{Comparison of INHES project results with INRAN's study results of 2005-06}

Despite the fact that INRAN's latest published data on food group consumption were based on a different food classification system (i.e. self-developed) [9], it is still very important to record the major differences of them compared to the present results. This comparison will take into account the variability evolved in food group consumption during the last decade in Italy.

Considering the adult populations (i.e. 18-65 years) both male and female INHES participants reported higher intakes of meat and meat products and lower consumption of "fish and seafood, amphibians, reptiles and invertebrates", "coffee, cocoa, tea and infusions" and "alcoholic beverages" compared to the INRAN populations [9]. This finding confirms in part the hypothesis that Italians tend to shift their dietary habits over the last years out of the traditional Mediterranean diet.
Studying the latest guidelines published by the World Health Organization [25] for the restriction in red meat and processed red meat intake, the observed increase in "meat and meat products" consumption over time is of particular concern in a public health perspective for the prevention of chronic diseases.

It is known that a concept of low carbohydrate and high protein diet against obesity in Italy has emerged [6-8]. This could also in part explain the increase in the consumption of "meat and meat products" while these food groups are the main sources of protein in such dietary patterns.

On the other hand, according to our data, other important protein sources such as "fish and seafood, amphibians, reptiles and invertebrates" seemed to be less consumed over time by both women and men. Several studies [26,27] have strengthened the importance of fish intake in the primary and secondary prevention of cardiovascular disease especially due to their high content in $n-3$ polyunsaturated fatty acids. The latter have multiple health protective biological actions in human metabolism $[26,28,29]$ and their high intake is important for public health promotion.

Furthermore, the decrease in "coffee, cocoa and tea and infusions" consumption is also of some concern, since they are major sources of antioxidant phytochemicals compounds, especially polyphenols. The latter have been recently studied for their role in modulating low-grade inflammation [30,31] and disease prevention [32].

On the other hand, quite optimistic findings are the higher consumption of fruits, legumes and nuts observed in the female INHES adult population compared with the corresponding consumption of female INRAN participants [9].

Focusing on the elderly women INHES population (i.e. $>65$ years), the intake of "grain and grain-based products", "milk and dairy products", "egg and eggs products" and "coffee, cocoa, tea and infusions" was lower than the corresponding INRAN participants. The decrease in such food groups over time is critical considering the special needs of the elderly for energy, protein and antioxidants [33]. Interestingly, meat and meat products and alcoholic beverages were higher in both elderly women and men within the INHES population compared to INRAN participants [9]. This needs further study in relation to the development and progression of chronic diseases.

\section{Differences in food group consumption in INHES population among different strata}

The variations observed in food group consumption between genders, geographical regions and educational level groups in the INHES population were interesting, too.

Among gender various differences, the higher intake of "meat and meat products" in men and the lower intake of "grains and grain-based products" in women were notable.

Interestingly, participants from South Italy reported greater consumption of "meat and meat products" while only North sample population had greater intake of "coffee, cocoa, tea and infusions".

In addition, participants at the highest educational level group had higher intake of "fruit and vegetable juices and 
nectars", "coffee, cocoa, tea and infusions" and "products for non-standard diets, food imitates and food supplements".

In general, the investigation of variations in food group intake between genders, educational level groups or geographical regions gain important meaning in public health perspectives and applied nutrition policy. Differences in food group intake could result in variations also in the dietary patterns of population groups.

Regarding gender differences, men naturally have greater needs for energy and nutrients compared to women. This fact, in combination with other lifestyle, socio-economic or even clinical factors (alteration of diet due to the presence of a disease) could result in significant gender variations.

In addition, geographical differences in food group intake could be mainly attributed to the socio-economic variations presented between Northern and Southern regions Italy but also on other factors such as culinary traditions that have a historical character.

Furthermore, the presented variations among educational groups confirm somehow the recent study of our research group on the impact of socio-economic status on dietary habits $[7,34]$. In fact, the analysis of data from 23,000 women and men participants of the Moli-sani cohort, living in the Molise region in Italy, showed that higher socio-economic status, especially greater income, was associated with healthier food choices through the adherence to the Mediterranean diet [34]. In addition, within the same population it was observed that at the time of economic crisis in Italy (i.e. 2007-2010), people with higher income or at higher educational level seemed to adhere better to the Mediterranean diet [7].

All together, these considerations underline the need for further investigation of factors affecting differences among various strata.

Furthermore, interesting results were derived from the analysis of the distribution of food group intake among meals. Notably, both women and men seemed to avoid or to eat less "vegetable and vegetable products", "nuts, oil seeds and spices", "fruit and fruit products" and "fruit and vegetable juices and nectars" during breakfast or snack meals.

On the other hand, "milk and dairy products", "eggs and egg products", "coffee, cocoa and tea and infusions", "products for non-standard diets, food imitates and food supplements" were less consumed for lunch and dinner.

The systematic absence of vegetables, fruits and nuts from breakfast or snack needs further attention considering the health benefits offered by their consumption in the prevention of chronic diseases [35,36]. In addition, the inclusion of "milk, dairy products" and "eggs and egg products" as lunch or dinner choices in combination with other food groups could result in an appropriate intake of protein, vitamin D and calcium especially in populations with special requirements [33]. It has also to be noted that the pattern of consuming "eggs and egg products" mainly for breakfast meal may be considered as a westernized influence on Italian dietary patterns.

\section{Comparison of INHES project results with EPIC study's EU} means

The comparison of the food group consumption data of the present work with the corresponding EU means may be relevant if one considers that they come from Italy, i.e. from a modern Mediterranean population.

Available accurate data on EU pooled means of food group consumption collected in different country samples (Spain, Greece, Italy, France, Germany, The Netherlands, United Kingdom, Denmark, Sweden and Norway) has been provided by the EPIC study [23]. Both women and men within the INHES population presented higher mean intakes of fruits, vegetables, vegetable oils and red or white meat and lower intake of processed meat and milk.

It is important to point out that the methodology of extracted food group data in INHES and EPIC studies differs especially in terms of the food classification system that was elaborated in each case. This limits somehow the accurate comparison of data while stresses the need for harmonized efforts in food group consumption evaluation and analysis.

\section{Strengths}

Among significant points of novelty and originality were the dietary assessment methodologies used, the INHES sample size and the elaboration of the FoodEx2 food classification for the first time in a EU population. The stratification of the descriptive analysis by gender, age group, geographical area, educational level and meal distribution illustrated in a more specific way the results for different study populations.

\section{Limitations}

Beyond the relevance of the findings of the present work, limitations do exist. First, bias of over- or under-estimation by the use of the dietary methodologies and for the extracted data should be acknowledged. In addition, two 24-HDRs of two non-consecutive days are appreciated as a homogeneous dietary assessment methodology for adults in EU. The one 24-HDR could not be considered as representative of the dietary habits of an individual and may widow the results not comparable with other European countries. Furthermore, the telephone-based interviews are under the limitation of misreporting or under/overestimation of food consumption.

\section{Conclusions}

In conclusion, this study presents the most recent data on food group consumption in an Italian sample. The data on the consumption of more than 70 food groups and subgroups that were studied and illustrated by gender, age group, geographical region and educational level group or type of meal, could be considered as a good source of information for future nutrition research in Italy and in EU. 


\section{Conflict of interest}

None declared.

\section{Acknowledgments}

The INHES Project was funded by Barilla and by the Italian Ministry of Economic Development within the frame of the ATENA program MI01_00093 - New Technologies for Made in Italy (D.I. PII MI 6/3/2008).

The funders had no role in study design, collection, analysis, and interpretation of data; in the writing of manuscripts and in the decision to submit the article for publication. All Authors were and are independent from funders.

\section{Appendix}

\section{INHES Investigators}

Principal Investigator: Licia Iacoviello.

Study coordinator: Americo Bonanni.

Scientific Committee: Marialaura Bonaccio, Americo

Bonanni, Chiara Cerletti, Simona Costanzo, Augusto Di Castelnuovo, Licia Iacoviello, Mariarosaria Persichillo, Maria Benedetta Donati, Giovanni de Gaetano.

Dietary questionnaire validation: Mariarosaria Persichillo and Francesco Zito.

Questionnaire administration: Lucia Aurisano, Paola Barisciano, Valentina Bonaccio, Francesca Bracone, Francesca De Lucia, Giovanna Galuppo, Filippo Petrucci, Anna Sciarretta, Angelita Verna.

Data management and analysis: Simona Costanzo, Augusto Di Castelnuovo, Marco Olivieri, George Pounis.

\section{Appendix A. Supplementary data}

Supplementary data related to this article can be found at http://dx.doi.org/10.1016/j.numecd.2017.01.004.

\section{References}

[1] Keys A, Taylor HL, Blackburn H, Brozek J, Anderson JT, Simonson E. Coronary heart disease among Minnesota business and professional men followed fifteen years. Circulation 1963;28:381-95.

[2] Nestle M. Mediterranean diets: historical and research overview. Am J Clin Nutr 1995;61:1313S-20S.

[3] de Lorgeril M, Salen P, Martin JL, Monjaud I, Delaye J, Mamelle N. Mediterranean diet, traditional risk factors, and the rate of cardiovascular complications after myocardial infarction: final report of the Lyon Diet Heart Study. Circulation 1999;99:779-85.

[4] Sofi F, Cesari F, Abbate R, Gensini GF, Casini A. Adherence to Mediterranean diet and health status: meta-analysis. BMJ 2008; $337: 1344$.

[5] Sofi F, Innocenti G, Dini C, Masi L, Battistini NC, Brandi ML, et al. Low adherence of a clinically healthy Italian population to nutritional recommendations for primary prevention of chronic diseases. Nutr Metab Cardiovasc Dis 2006;16:436-44.

[6] Pounis G, Castelnuovo AD, Costanzo S, Persichillo M, Bonaccio M, Bonanni A, et al. Association of pasta consumption with body mass index and waist-to-hip ratio: results from Moli-sani and INHES studies. Nutr Diabetes 2016;6:e218.

[7] Bonaccio M, Di Castelnuovo A, Bonanni A, Costanzo S, De Lucia F, Persichillo M, et al. Decline of the Mediterranean diet at a time of economic crisis. Results from the Moli-sani study. Nutr Metab Cardiovasc Dis 2014 Aug;24(8):853-60. http://dx.doi.org/10.1016/j.numecd.2014.02.014. Epub 2014 Mar 1.

[8] Sette S, Le Donne C, Piccinelli R, Mistura L, Ferrari M, Leclerca C INRAN-SCAI 2005-06 study group. The third National Food Consumption Survey, INRAN-SCAI 2005-06: major dietary sources of nutrients in Italy. Int J Food Sci Nutr 2013;64:1014-21.

[9] Leclercq C, Arcella D, Piccinelli R, Sette S, Le Donne C, Turrini A, INRAN-SCAI 2005-06 Study Group. The Italian National Food Consumption Survey INRAN-SCAI 2005-06: main results in terms of food consumption. Public Health Nutr 2009;12:2504-32.

[10] European Food Safety Authority. Technical Report, The food classification and description system FoodEx2 (revision 2). Last accessed, May 2016 https://www.efsa.europa.eu/sites/default/ files/scientific_output/files/main_documents/804e.pdf.

[11] Giampaoli S, Palmieri L, Donfrancesco C, Noce CL, Pilotto L, Vanuzzo D, Osservatorio Epidemiologico Cardiovascolare/Health Examination Survey Research Group. Cardiovascular health in Italy. Ten-year surveillance of cardiovascular diseases and risk factors: Osservatorio Epidemiologico Cardiovascolare/Health Examination Survey 1998-2012. Eur J Prev Cardiol 2015;22:9-37.

[12] Laurendi G, Donfrancesco C, Palmieri L, Vanuzzo D, Scalera G, Giampaoli S, Osservatorio Epidemiologico Cardiovascolare/Health Examination Survey 2008-2012. Association of lifestyle and cardiovascular risk factors with lung function in a sample of the adult Italian population: a cross-sectional survey. Respiration 2015;89: 33-40.

[13] Illner AK, Harttig U, Tognon G, Palli D, Salvini S, Bower E, et al. Feasibility of innovative dietary assessment in epidemiological studies using the approach of combining different assessment instruments. Public Health Nutr 2011 Jun;14(6):1055-63. http: //dx.doi.org/10.1017/S1368980010003587. Epub 2011 Mar 9.

[14] Gandek B, Ware JE, Aaronson NK, Apolone G, Bjorner JB, Brazier JE, et al. Cross- validation of item selection and scoring for the SF-12 health survey in nine countries: results from the IQOLA project. J Clin Epidemiol 1998;51:1171-8.

[15] Van Kappel AL, Amoyel J, Slimani N, Vozar B, Riboli E. EPIC-SOFT picture book for estimating portion sizes. Lyon, France: IARC Press; 1994.

[16] Sette S, Le Donne C, Piccinelli R, Arcella D, Turrini A, Leclercq C, INRAN-SCAI 2005-6 Study Group. The third Italian National Food Consumption Survey, INRAN-SCAI 2005-06-part 1: nutrient intakes in Italy. Nutr Metab Cardiovasc Dis 2011;21:922-32.

[17] Agnoli C, Krogh V, Grioni S, Sieri S, Palli D, Masala G, et al. A prioridefined dietary patterns are associated with reduced risk of stroke in a large Italian cohort. J Nutr 2011;141:1552-8.

[18] European Food Safety Authority. Exposure hierarchy FoodEx2 (revision 2). Last accessed, May 2016 https://www.efsa.europa.eu/ sites/default/files/assets/ExposureHierarchyrevision2.zip.

[19] de Boer EJ, Slimani N, van 't Veer P, Boeing H, Feinberg M, Leclercq C, et al., EFCOVAL Consortium.. The European food consumption validation project: conclusions and recommendations. Eur J Clin Nutr 2011 Jul;65(Suppl. 1):S102-7. http: //dx.doi.org/10.1038/ejcn.2011.94.

[20] de Boer EJ, Slimani N, van 't Veer P, Boeing H, Feinberg M, Leclercq C, et al., EFCOVAL Consortium. Rationale and methods of the European food consumption validation (EFCOVAL) project. Eur J Clin Nutr 2011 Jul;65(Suppl. 1):S1-4. http://dx.doi.org/10.1038/ejcn.2011.82.

[21] Razali NM, Wah YB. Power comparisons of Shapiro-Wilk, Kolmogorov-Smirnov, Lilliefors and Anderson-Darling tests. J Stat Model Anal $2011 ; 2: 21-33$.

[22] Saba A, Turrini A, Mistura G, Cialfa E, Vichi M. Indagine nazionale sui consumi alimentari delle famiglie 1980-84: alcuni principali risultati (Nation-wide survey on Italian households food consumption 1980-84: main results). J It Soc Food Sci 1990;1990(19): 53-65.

[23] Slimani N, Fahey M, Welch AA, Wirfält E, Stripp C, Bergström E, et al. Diversity of dietary patterns observed in the European prospective investigation into cancer and nutrition (EPIC) project. Public Health Nutr 2002;5:1311-28.

[24] Turrini A, Saba A, Perrone D, Cialfa E, D'Amicis A. Food consumption patterns in Italy: the INN-CA Study 1994-96. Eur J Clin Nutr 2001;55:571-88.

[25] Bouvard V, Loomis D, Guyton KZ, Grosse Y, Ghissassi FE, Benbrahim-Tallaa L, et al., International agency for Research on Cancer 
Monograph Working Group. Carcinogenicity of consumption of red and processed meat. Lancet Oncol 2015 Dec;16(16):1599-600. http://dx.doi.org/10.1016/S1470-2045(15)00444-1. Epub 2015 Oct 29.

[26] Kris-Etherton P, Harris W, Appel L, For the Nutrition Committee. Fish consumption, fish oil, $\mathrm{n}-3$ fatty acids, and cardiovascular disease. Circulation 2002:106:2747-57.

[27] GISSI Prevention trial. Dietary supplementation with n-3 polyunsaturated fatty acids and vitamin E after myocardial infarction: results of the GISSI-Prevenzione trial. Lancet 1999:354:447-55.

[28] Chrysohoou C, Panagiotakos DB, Pitsavos C, Skoumas J, Krinos X Chloptsios Y, et al. Long-term fish consumption is associated with protection against arrhythmia in healthy persons in a Mediterranean region-the ATTICA study. Am J Clin Nutr 2007;85:1385-91.

[29] Kristensen SD, Iversen AM, Schmidt EB. N-3 polyunsaturated fatty acids and coronary thrombosis. Lipids 2001:36(Suppl. 1):S79-82.

[30] Pounis G, Bonaccio M, Di Castelnuovo A, Costanzo S, de Curtis A Persichillo $\mathrm{M}$, et al. Polyphenol intake is associated with lowgrade inflammation, using a novel data analysis from the Molisani study. Thromb Haemost 2016 Jan;115(2):344-52. http: //dx.doi.org/10.1160/TH15-06-0487. Epub 2015 Sep. 10.
[31] Pounis G, Costanzo S, di Giuseppe R, de Lucia F, Santimone I, Sciarretta A, et al. Consumption of healthy foods at different content of antioxidant vitamins and phytochemicals and metabolic risk factors for cardiovascular disease in men and women of the Moli-sani study. Eur J Clin Nutr 2013 Feb;67(2):207-13. http: //dx.doi.org/10.1038/ejcn.2012.201. Epub 2012 Dec 19.

[32] Pandey KB, Rizvi SI. Plant polyphenols as dietary antioxidants in human health and disease. Oxid Med Cell Longev 2009;2:270-8.

[33] Hellwig JP, Meyers LD, Otten JJ. Dietary reference intakes: the essential guide to nutrient requirement. National Academies Press; 2006.

[34] Bonaccio M, Bonanni AE, Di Castelnuovo A, De Lucia F, Donati MB, de Gaetano G, et al., Moli-sani Project Investigators. Low income is associated with poor adherence to a Mediterranean diet and a higher prevalence of obesity: cross-sectional results from the Moli-sani study. BMJ Open 2012 Nov 19;2(6). http://dx.doi.org/10.1136/bmjopen-2012-001685. pii: e001685; Print 2012.

[35] Slavin JL, Lloyd B. Health benefits of fruits and vegetables. Adv Nutr 2012 Jul 1;3(4):506-16. http://dx.doi.org/10.3945/an.112.002154.

[36] Ros E. Health benefits of nut consumption. Nutrients $2010 \mathrm{Jul}$; 2(7):652-82 


\section{CHAPTER 3.}

Consumption of whole grain food and its determinants in a general Italian population: Results from the INHES study

Ruggiero E, Bonaccio M, Di Castelnuovo A, Bonanni A, Costanzo S, Persichillo M, Bracone F, Cerletti C, Donati MB, de Gaetano G, Iacoviello L; INHES Study Investigators

Nutr Metab Cardiovasc Dis. 2019 Jun;29(6):611-620. 



\title{
Consumption of whole grain food and its determinants in a general Italian population: Results from the INHES study
}

\author{
E. Ruggiero a , M. Bonaccio a,*, A. Di Castelnuovo ${ }^{\text {b }}$, A. Bonanni ${ }^{\text {a }}$, S. Costanzo a, \\ M. Persichillo ${ }^{a}$, F. Bracone ${ }^{a}$, C. Cerletti ${ }^{a}$, M.B. Donati ${ }^{a}$, G. de Gaetano ${ }^{a}$, L. Iacoviello ${ }^{a, c}$ \\ on behalf of the INHES Study Investigators ${ }^{1}$ \\ ${ }^{a}$ Department of Epidemiology and Prevention, IRCCS Neuromed, 86077, Pozzilli (IS), Italy \\ ${ }^{b}$ Mediterranea Cardiocentro, Napoli, Italy \\ ${ }^{c}$ Department of Medicine and Surgery, Research Center in Epidemiology and Preventive Medicine (EPIMED), University of Insubria, 21100, Varese, Italy \\ Received 2 October 2018; received in revised form 11 February 2019; accepted 1 March 2019 \\ Handling Editor: F. Galletti \\ Available online 12 March 2019
}

\section{KEYWORDS}

Whole grain;

General population;

Socioeconomic

factors;

Psychosocial factors;

Meal patterns

\begin{abstract}
Background and aims: Whole grain (WG) food consumption is associated with lower risk of cardiovascular disease, cancer and neurological diseases. The aim of this study was to assess the consumption of WG food and its major demographic, socioeconomic, psychosocial and behavioral determinants in a general Italian population.

Methods and results: Data were from the Italian Nutrition \& Health Survey (INHES), a telephonebased survey established in 2010-2013 including 9422 participants aged $\geq 5$ years from all over Italy. WG food intake was assessed by the European Food Propensity Questionnaire and included bread, pasta, breakfast cereals, biscuits and WG soups. WG consumption was categorized as none, occasional ( $<1$ time/week) and regular ( $\geq 1$ time/week). Overall, $26.9 \%$ of the sample reported a regular consumption of WG food $(27.2 \%$ of adults aged $20-97 \mathrm{y}$, and $21.9 \%$ of children/adolescents aged 5-19 y). In both age-groups, the major food source contributing to total WG intake was WG bread followed by WG pasta. Among adults, greater consumption of WG was associated with healthier lifestyle (e.g. sport activity), and higher educational level. Eating meals outside of the house in adults, and spending $>2 \mathrm{~h} /$ day watching TV in children/adolescents were inversely associated with WG intake.

Conclusions: The percentage of WG consumers in Italy in 2010-2013 appears to be quite low and still below that recorded in other countries of Europe where consumption is frequently over 50 percent. WG consumption is likely to be influenced by socioeconomic status and is associated with a number of psychosocial factors, meal patterns and eating-related behaviors.

( 2019 The Italian Society of Diabetology, the Italian Society for the Study of Atherosclerosis, the Italian Society of Human Nutrition, and the Department of Clinical Medicine and Surgery, Federico II University. Published by Elsevier B.V. All rights reserved.
\end{abstract}

\footnotetext{
* Corresponding author. Department of Epidemiology and Prevention, IRCCS Neuromed, Via dell'Elettronica, 86077, Pozzilli (IS), Italy.

E-mail addresses: marialaura.bonaccio@moli-sani.org, marialaura. bonaccio@neuromed.it (M. Bonaccio).

${ }^{1}$ INHES Study Investigators are listed in the Appendix.
}

\section{Introduction}

In numerous epidemiological studies consumption of whole grain (WG) products is associated with lower risk of chronic diseases such as coronary heart disease [1], stroke [2], type 2 diabetes [3] and colon-rectal cancer [4]. Moreover, habitual consumption of WG foods may contribute to 
improved adiposity indices in children and adults [5] and appears to exert benefits also for cardiovascular risk markers, such as blood pressure, insulin sensitivity and plasma cholesterol [6,7]. WG consumption of any population is difficult to study, as presently, there is no universally accepted definition of WG [8].

Recently, the Health Grain Forum, a not-for-profit consortium of academics and industry working with cereal foods, suggested that a food may be labeled as "whole grain" if it contains $>30 \%$ whole-grain ingredients in the overall product and more whole grain than refined grain ingredients, both on a dry-weight basis [9]. Moreover, countries including the USA, Canada, Denmark and Australia have specific dietary guidelines on WG intake but others, as Italy, do not explicitly indicate the amount of WG to be consumed, rather they only suggest to regularly consume cereals preferably whole [10].

In particular, in the US the recommended daily consumption is of at least $85 \mathrm{~g} / \mathrm{d}$ [11] for adults and $42 \mathrm{~g} / \mathrm{d}$ for children [12], while in Denmark is set at $75 \mathrm{~g} / 10 \mathrm{MJ}$ (2388 kcal) per day [13].

The Mediterranean diet pyramid recommends the consumption of one or two servings of cereals at each main meal (in the form of bread, pasta, rice, couscous and others), preferably whole grain [14].

The latest updated data on WG consumption in Italy derive from the INRAN-SCAI 2005-06, a cross-sectional survey conducted on a representative sample of 1300 households randomly selected and stratified into the four main geographical areas of Italy (North-West, North-East, Centre, South and Islands). This study showed that WG products, over the 3-day survey period, were consumed in only a quarter of Italian children and adults, with mean intakes being substantially lower than quantitative WG recommendations [15].

The aim of this study was twofold: first, to assess the WG intake in a large sample of the Italian population recruited all over Italy within the Italian Nutrition \& Health Survey (INHES); second, to investigate major demographic, socioeconomic, psychosocial and behavioral factors possibly associated with the consumption of WG foods, in order to provide new evidence for appropriate intervention strategies at population level.

\section{Methods}

\section{Study population}

The INHES Study is a 3-year telephone-based survey on nutrition and health specifically designed to collect information on dietary habits (quality, quantity and meal patterns), food choice determinants, and food health awareness of the Italian population according to different geographical distribution (Northern, Central and Southern Italy), age, gender and socioeconomic status.

Between November 2010 and November 2013, 9422 women and men aged $\geq 5$ years from all over Italy were enrolled. A full description of the study design is reported in the supplementary material.
For the purpose of the present study, we omitted subjects with missing information on WG consumption (3.1\%) or missing values for major variables of interest (1.6\%). A total of 8421 adults (aged $>19 \mathrm{y}$ ) and 561 young subjects (aged $\leq 19 \mathrm{y}$ ) were finally included in the analyses.

The INHES Study was conducted according to the guidelines laid down in the Declaration of Helsinki and all procedures involving human subjects were approved by the Ethical Committee of the Catholic University of Rome. Verbal informed consent was obtained from all subjects and was witnessed and formally recorded.

\section{Dietary assessment}

Data on food intake were collected through the Italian version of the European Food Propensity Questionnaire according to methods described elsewhere [16].

Total WG food intake was estimated by using information from five different WG food groups: WG bread, WG pasta, WG breakfast cereals, WG biscuits (including also crackers and breadsticks) and WG soups. For each food item, frequency of consumption was collected by the following question: "How frequently do you eat (e.g.) WG pasta?" and possible answers were: 'never', 'once a month or less frequent', 'less than 3 times a month', '2-3 times/month', ' $1-2$ times a week', '3-4 times a week', '5-6 times a week', 'every day', 'more than 1 time/day'. The total consumption of WG was obtained by summing the frequency (converted into times/week) of consumption of each WG food group. The study population was eventually categorized as non-WG food consumers, consuming WG $<1$ time/week and consuming WG $\geq 1$ time/week.

Adherence to the Mediterranean diet in the adult sample was evaluated by using the MedDietScore as proposed by Panagiotakos [17] including 10 food groups (fruits, vegetables, legumes, potatoes, fish, red meat, poultry, dairy products, alcoholic beverages, olive oil). As compared to the original score, we did not include WG cereals.

The final score potentially ranged from 0 to 50 , the latter reflecting maximal adherence. For analysis purposes, the score was also used as exact tertiles.

Adherence to the Mediterranean diet in the young sample was evaluated by using the KIDMED index (Mediterranean Diet Quality Index) for children and teenagers [18], classified as follows: good ( $\geq 6$ points, indicating an optimal Mediterranean diet); average ( $4-5$ points); and poor ( $\leq 3$ points).

The reasons for consumption of WG products was assessed through the question "What do you think about WG products?" with four possible answers ('they are healthier than regular ones', 'they are more natural', 'other,' 'do not know'). Data on reasons for nonconsumption of WG was obtained by the question "Why don't you eat WG products?" with four possible answers ('dislike', 'lack of knowledge', 'hard to cook' or 'other'). The questionnaire on consumption of WG was administered to a random subgroup of the sample $(n=5805)$. 


\section{Meal patterns}

Meal patterns comprised either patterning of main meals (breakfast, lunch, dinner) or context of main meals, such as meals eaten out of the home, or eating meals in front of the television or when using PC. Information on daily amount of time spent in watching TV/using PC was also collected and categorized as $\leq$ or $>2 \mathrm{~h} / \mathrm{d}$.

\section{Socioeconomic and psychosocial factors}

Education was based on the highest qualification attained and was categorized as up to primary school (corresponding to $\leq 5$ years of study), lower secondary ( $>5 \leq 8$ years), upper secondary ( $>8 \leq 13$ years), post-secondary ( $>13$ years).

Present occupation was assembled into 6 groups: manual, non-manual, housewife, retired, student and unemployed. Marital status was categorized as married/living in a couple, single, separated/divorced and widowed.

Self-rated health was assessed by the question "How would you rate your health status" and possible answers ranged from 'very good' to 'bad' [19]. Adverse life events (yes/no) during the last year included questions related to health (e.g. serious illness or death), job (e.g. retired, job loss), or interpersonal situations (e.g. divorce, problem with friend or relative).

Information on occupational and family-related stress was obtained by the question "How often have you experienced stress in your workplace/family?" with answers ranging from 'never' to 'always'. Financial-related stress was rated as none/little, moderate and a lot. Job control was considered as none/little, moderate-good, fairly good and not working/unascertained.

\section{Assessment of covariates}

Previous diagnosis of diabetes, hypercholesterolemia, hypertension, cardiovascular disease and cancer was selfreported and categorized as no/yes. In adults, body mass index was calculated by using self-reported measurements of height and weight, calculated as $\mathrm{kg} / \mathrm{m}^{2}$ and grouped into three categories as normal $(<25)$, overweight $(\geq 25<30)$ or obese $(\geq 30)$. BMI in children/adolescents was categorized according to specific values for children considering sex and age [20]. Engagement in sport activity was considered as a dichotomous variable (yes/no).

\section{Statistical analysis}

Main characteristics of the study population across categories of weekly WG food consumption were presented as means with standard deviation (SD), or percentages.

Associations for categorical or continuous variables were evaluated by using general linear models adjusted for age groups (see Table 1 for definition of age groups) and sex (PROC GENMOD and PROC GLM in SAS for categorical and continuous variables, respectively).

Using multinomial ordered multivariable logistic regression analysis, odds ratios (ORs) with corresponding
95\% confidence intervals $(95 \% \mathrm{CI})$ were calculated to estimate the association of 3-level WG consumption (none vs occasional vs regular) with demographic and socioeconomic factors (Tables 2 and 3), meal patterns and eatingrelated behaviours (Table 4) or psychosocial factors (Table 5).

Dummy variables for missing values of each variable of interest were created. Two-sided P-value $<0.05$ was considered as statistically significant.

The data analysis was generated using SAS/STAT software, Version 9.4 of the SAS System for Windows ${ }^{\odot} 2009$. SAS Institute Inc. and SAS are registered trademarks of SAS Institute Inc., Cary, NC, USA.

\section{Results}

\section{Adults}

Overall, $27.2 \%$ of adults (20-97 y) reported a regular consumption ( $\geq 1$ time/week) of WG products (Table 1 ).

Among adults, regular WG intake was more prevalent in middle-aged subjects (50-64 years), in women and in subjects from Northern Italy and within those having a higher educational level (Table 1). Moreover, regular WG consumption was higher in adults with a non-manual occupation, and among single or separated/divorced, non-smokers, and for those engaged in some physical activity or with normal body weight (Table 1). Adherence to the Mediterranean diet was slightly higher in nonconsumer/occasional consumers (Table 1 ).

Supplementary Figure $1 \mathrm{~A}$ shows the percent contribution from five different WG food sources, (i.e. bread, pasta, cereals, biscuits, soup) to the total WG intake. The major food sources were represented by WG bread (53.3\%) and WG biscuits (27.4\%), followed by WG pasta (13.1\%), while only $4.8 \%$ and $1.3 \%$ were contributed by WG breakfast cereals and WG soups, respectively.

Table 2 reports the association of demographic and socioeconomic factors with WG consumption in adult subjects, with occasional or regular WG food intake as opposed to non-consumers.

A clear educational gradient in WG intake emerged, with subjects with highest educational attainment (postsecondary) reporting higher odds of being either occasional $(\mathrm{OR}=1.90 ; 95 \% \mathrm{CI} 1.58-2.27)$ or regular consumers $(\mathrm{OR}=1.59 ; 1.30-1.95)$. WG intake was also related to occupation, marital status and engagement in sport activities (Table 2).

Having breakfast out of home $(\mathrm{OR}=0.59 ; 0.46-0.76$ and $\mathrm{OR}=0.43 ; 0.31-0.60$, for occasional and regular WG consumption, respectively) was inversely associated with WG food intake, as compared to having breakfast at home, and the same was true for individuals having lunch out of home and for those having meals while watching TV (Table 4; Model 2b).

Finally, poor self-reported health status was inversely associated with regular WG food intake $(\mathrm{OR}=0.44$; $0.38-0.51)$ as well as moderate financial circumstances- 
Table 1 Characteristics of the adult population (20-97 years) according to consumption of whole grain foods

\begin{tabular}{|c|c|c|c|c|c|}
\hline & \multicolumn{5}{|c|}{ Whole grain products intake } \\
\hline & Whole sample & Non-consumers & $\begin{array}{l}\text { Occasional consumers } \\
(<1 \text { times/week })\end{array}$ & $\begin{array}{l}\text { Regular consumers } \\
\text { ( } \geq 1 \text { times/week) }\end{array}$ & Unadjusted $p$ value \\
\hline $\mathrm{N}(\%)$ & $8421(100)$ & $4656(55.3)$ & $1475(17.5)$ & $2290(27.2)$ & - \\
\hline Age groups (y) & & & & & $<0.0001$ \\
\hline $20-34$ & 6.4 & 6.6 & 6.9 & 5.6 & \\
\hline $35-49$ & 28.4 & 26.3 & 31.7 & 30.8 & \\
\hline $50-64$ & 33.0 & 30.9 & 37.2 & 34.6 & \\
\hline $65-74$ & 20.8 & 22.7 & 17.6 & 18.9 & \\
\hline$\geq 75$ & 11.4 & 13.5 & 6.6 & 10.0 & \\
\hline Sex & & & & & $<0.0001$ \\
\hline Women & 53.3 & 47.5 & 58.8 & 61.3 & \\
\hline Men & 46.7 & 52.5 & 41.2 & 38.7 & \\
\hline Geographical area & & & & & $<0.0001$ \\
\hline Northern & 40.5 & 39.8 & 36.9 & 44.2 & \\
\hline Central & 16.7 & 16.1 & 20.3 & 15.5 & \\
\hline Southern & 42.8 & 44.1 & 42.8 & 40.3 & \\
\hline Educational level & & & & & $<0.0001$ \\
\hline Up to primary & 17.7 & 21.1 & 12.1 & 14.1 & \\
\hline Lower secondary & 26.3 & 28.1 & 24.7 & 23.8 & \\
\hline Upper secondary & 39.8 & 38.0 & 42.3 & 41.8 & \\
\hline Post-secondary & 16.2 & 12.8 & 20.9 & 20.3 & \\
\hline Occupation & & & & & $<0.0001$ \\
\hline Non-manual & 31.1 & 26.2 & 37.6 & 36.7 & \\
\hline Manual & 18.0 & 19.1 & 18.0 & 15.9 & \\
\hline Housewife & 11.0 & 10.9 & 11.5 & 10.8 & \\
\hline Retired & 35.2 & 38.8 & 29.1 & 31.9 & \\
\hline Student & 1.8 & 1.9 & 1.7 & 1.6 & \\
\hline Unemployed & 2.9 & 3.1 & 2.1 & 3.1 & \\
\hline Marital status & & & & & $<0.0001$ \\
\hline Married/in couple & 75.1 & 75.3 & 75.3 & 74.5 & \\
\hline Single & 14.6 & 13.9 & 15.9 & 15.4 & \\
\hline Separated/divorced & 3.2 & 2.5 & 3.5 & 4.5 & \\
\hline Widowed & 7.1 & 8.3 & 5.3 & 5.6 & \\
\hline Smoking habit & & & & & $<0.0001$ \\
\hline No & 59.7 & 56.2 & 59.7 & 66.8 & \\
\hline Yes & 15.9 & 17.9 & 15.2 & 12.4 & \\
\hline Former & 22.4 & 23.9 & 23.3 & 18.6 & \\
\hline Occasional & 2.0 & 2.0 & 1.8 & 2.2 & \\
\hline Sport activity & & & & & $<0.0001$ \\
\hline No & 81.6 & 84.3 & 78.8 & 77.8 & \\
\hline Yes & 18.4 & 15.7 & 21.2 & 22.2 & \\
\hline Body mass index & & & & & 0.0015 \\
\hline Normal weight & 48.1 & 46.5 & 48.1 & 51.4 & \\
\hline Overweight & 38.3 & 39.1 & 38.6 & 36.6 & \\
\hline Obese & 13.6 & 14.4 & 13.3 & 12.0 & \\
\hline Cardiovascular disease & 3.2 & 3.9 & 2.0 & 2.7 & 0.0002 \\
\hline Cancer & 3.4 & 3.4 & 3.1 & 3.7 & 0.66 \\
\hline Hypertension & 31.8 & 33.2 & 28.4 & 31.2 & 0.0023 \\
\hline Diabetes & 7.6 & 7.3 & 7.2 & 8.4 & 0.22 \\
\hline Hypercholesterolemia & 21.9 & 21.6 & 21.4 & 22.7 & 0.49 \\
\hline MedDietScore ${ }^{a}$ & 28.7 (3.6) & $28.8(3.5)$ & 28.9 (3.5) & $28.5(3.9)$ & 0.0046 \\
\hline
\end{tabular}

Values are reported as percentages.

a MedDietScore (not including whole grain foods) is reported as means with standard deviations.

related stress ( $\mathrm{OR}=0.55 ; 0.40-0.76)$, and higher familyrelated stress ( $\mathrm{OR}=0.59 ; 0.40-0.87$; Table 5 , Model 2).

\section{Children/adolescents}

Among children/adolescents (5-19 years), 21.9\% reported a regular consumption ( $\geq 1$ times/week) of WG products (Table 3).
In this sample, female gender and Southern Italy geographical area were major determinants of regular WG intake (Table 3).

In agreement with what observed for adults, the major food source was represented by WG bread (42.3\%), followed by WG biscuits (20.2\%), WG breakfast cereals (15.7\%) and WG pasta (15.8\%) whereas WG soups contributed to $6.0 \%$ of the total WG intake (Supplementary Figure $1 \mathrm{~A})$. 
Table 2 Demographic and socioeconomic factors associated with whole grain food consumption in the adult population (20-97 years).

\begin{tabular}{|c|c|c|c|c|}
\hline & \multicolumn{2}{|c|}{$\begin{array}{l}\text { Occasional consumers }(n=1475) \text { vs non- } \\
\text { consumers }(n=4656)\end{array}$} & \multicolumn{2}{|c|}{$\begin{array}{l}\text { Regular consumers }(\mathrm{n}=2290) \text { vs non-consumers } \\
(\mathrm{n}=4656)\end{array}$} \\
\hline & Model 1 OR (95\%CI) & Model 2 OR (95\%CI) & Model 1 OR (95\%CI) & Model 2 OR (95\%CI) \\
\hline \multicolumn{5}{|l|}{ Age groups (y) } \\
\hline $20-34$ & $-1-$ & $-1-$ & $-1-$ & $-1-$ \\
\hline $35-49$ & $1.28(1.06-1.54)$ & $1.44(1.13-1.84)$ & $1.34(1.08-1.66)$ & $1.70(1.29-2.23)$ \\
\hline $50-64$ & $1.24(1.03-1.50)$ & $1.59(1.23-2.05)$ & $1.28(1.03-1.58)$ & $1.84(1.38-2.45)$ \\
\hline $65-74$ & $0.89(0.73-1.08)$ & $1.32(0.99-1.77)$ & $1.07(0.85-1.34)$ & $1.75(1.26-2.44)$ \\
\hline$\geq 75$ & $0.70(0.56-0.87)$ & $1.21(0.88-1.66)$ & $1.03(0.80-1.32)$ & $1.91(1.34-2.72)$ \\
\hline \multicolumn{5}{|l|}{ Sex } \\
\hline Women & $-1-$ & $-1-$ & $-1-$ & $-1-$ \\
\hline Men & $0.60(0.55-0.65)$ & $0.58(0.51-0.63)$ & $0.63(0.57-0.70)$ & $0.63(0.56-0.70)$ \\
\hline \multicolumn{5}{|l|}{ Geographical area } \\
\hline Northern & $-1-$ & $-1-$ & $-1-$ & $-1-$ \\
\hline Central & $1.05(0.92-1.19)$ & $1.01(0.88-1.14)$ & $0.80(0.70-0.93)$ & $0.78(0.68-0.90)$ \\
\hline Southern & $0.87(0.79-0.96)$ & $0.92(0.84-1.02)$ & $0.80(0.72-0.89)$ & $0.85(0.76-0.95)$ \\
\hline \multicolumn{5}{|l|}{ Educational level } \\
\hline Up to primary & $-1-$ & $-1-$ & $-1-$ & $-1-$ \\
\hline Lower secondary & $1.32(1.14-1.53)$ & $1.33(1.15-1.55)$ & $1.25(1.05-1.47)$ & $1.25(1.06-1.49)$ \\
\hline Upper secondary & $1.69(1.46-1.95)$ & $1.50(1.29-1.74)$ & $1.53(1.30-1.80)$ & $1.40(1.18-1.66)$ \\
\hline Post-secondary & $2.36(1.99-2.80)$ & $1.90(1.58-2.27)$ & $1.93(1.60-2.32)$ & $1.59(1.30-1.95)$ \\
\hline \multicolumn{5}{|l|}{ Occupation } \\
\hline Non-manual & $-1-$ & $-1-$ & $-1-$ & $-1-$ \\
\hline Manual & $0.65(0.57-0.74)$ & $0.78(0.68-0.90)$ & $0.69(0.60-0.80)$ & $0.81(0.69-0.94)$ \\
\hline Housewife & $0.57(0.48-0.67)$ & $0.70(0.59-0.83)$ & $0.60(0.50-0.72)$ & $0.71(0.59-0.86)$ \\
\hline Retired & $0.68(0.58-0.79)$ & $0.80(0.69-0.94)$ & $0.71(0.60-0.85)$ & $0.83(0.69-0.98)$ \\
\hline Student & $0.75(0.51-1.10)$ & $0.80(0.54-1.19)$ & $0.94(0.60-1.46)$ & $0.97(0.61-1.54)$ \\
\hline Unemployed & $0.63(0.48-0.82)$ & $0.74(0.56-0.98)$ & $0.90(0.67-1.21)$ & $1.07(0.79-1.45)$ \\
\hline \multicolumn{5}{|l|}{ Marital status } \\
\hline Married/in couple & $-1-$ & $-1-$ & $-1-$ & $-1-$ \\
\hline Single & $1.19(1.03-1.39)$ & $1.14(0.98-1.33)$ & $1.19(1.01-1.40)$ & $1.14(0.97-1.35)$ \\
\hline Separated/divorced & $1.47(1.15-1.88)$ & $1.43(1.11-1.85)$ & $1.48(1.15-1.91)$ & $1.46(1.13-1.89)$ \\
\hline Widowed & $0.68(0.56-0.83)$ & $0.70(0.58-0.85)$ & $0.68(0.55-0.85)$ & $0.68(0.55-0.85)$ \\
\hline \multicolumn{5}{|l|}{ Smoking habit } \\
\hline No & $-1-$ & $-1-$ & $-1-$ & $-1-$ \\
\hline Yes & $0.65(0.58-0.74)$ & $0.66(0.58-0.76)$ & $0.62(0.53-0.72)$ & $0.62(0.53-0.72)$ \\
\hline Former & $0.90(0.80-1.00)$ & $0.90(0.80-1.01)$ & $0.76(0.66-0.86)$ & $0.75(0.66-0.86)$ \\
\hline Occasional & $0.90(0.66-1.23)$ & $0.85(0.62-1.17)$ & $0.99(0.71-1.40)$ & $0.93(0.66-1.32)$ \\
\hline \multicolumn{5}{|l|}{ Sport activity } \\
\hline No & $-1-$ & $-1-$ & $-1-$ & $-1-$ \\
\hline Yes & $1.48(1.32-1.66)$ & $1.34(1.19-1.51)$ & $1.41(1.25-1.60)$ & $1.32(1.16-1.49)$ \\
\hline \multicolumn{5}{|l|}{ Body mass index } \\
\hline Normal weight & $-1-$ & $-1-$ & $-1-$ & $-1-$ \\
\hline Overweight & $1.01(0.91-1.11)$ & $1.08(0.98-1.20)$ & $0.94(0.84-1.05)$ & $1.00(0.90-1.12)$ \\
\hline Obese & $0.87(0.76-0.99)$ & $0.99(0.86-1.14)$ & $0.81(0.69-0.94)$ & $0.90(0.76-1.05)$ \\
\hline \multicolumn{5}{|l|}{ MedDietScore } \\
\hline Poor (5-27) & $-1-$ & $-1-$ & $-1-$ & $-1-$ \\
\hline Average (28-30) & $0.87(0.78-0.97)$ & $0.85(0.76-0.95)$ & $0.81(0.71-0.91)$ & $0.80(0.71-0.91)$ \\
\hline Good $(31-44)$ & $1.00(0.90-1.11)$ & $0.97(0.87-1.08)$ & $0.86(0.76-0.96)$ & $0.86(0.76-0.97)$ \\
\hline
\end{tabular}

Model 1 = Odds ratio (OR) with $95 \%$ confidence intervals ( $95 \% \mathrm{CI}$ ) obtained from a model adjusted for age groups and sex.

Model 2 = as in model 1, further controlled for geographical area, educational level, occupation, marital status, smoking habit, sport activity, BMI,

CVD, hypertension and adherence to Mediterranean diet (not including whole grains).

Table 3 reports the association of demographic and socioeconomic factors with WG consumption among younger subjects, with WG food intake. Boys were less likely to consume WG as compared to girls $(\mathrm{OR}=0.59$; $0.41-0.86$ and $\mathrm{OR}=0.65 ; 0.43-1.01$, for occasional and regular consumption, respectively, Table 3; Model 2).

Younger consumers spending $>2 \mathrm{~h} / \mathrm{d}$ using $\mathrm{PC}$ were less likely to be occasional ( $\mathrm{OR}=0.53 ; 0.36-0.80$ ) or regular consumers $(\mathrm{OR}=0.56 ; 0.36-0.87$ ) (Table 4 ; Model 2).

\section{Determinants of consumers' food choice}

Supplementary Figure 2 shows the main reasons people choose to consume WG products. A great proportion of WG consumers believe WG food is healthy food (47.7\%) or more natural than regular products (26.2\%).

Reasons for non-consumption of WG are depicted in Supplementary Figure 3 and ranged from dislike (38.8\%) to an actual lack of knowledge on WG products (45.3\%), while about $12.9 \%$ believes WG food is 'hard to cook'. 


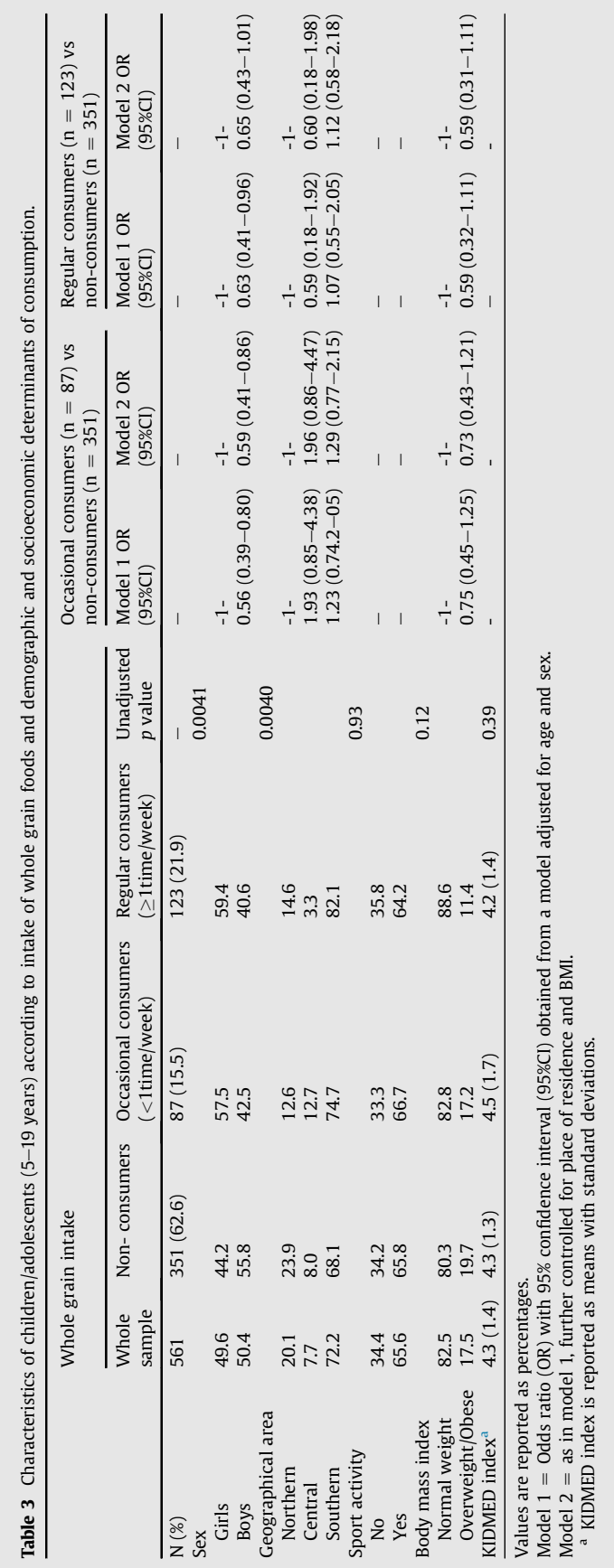




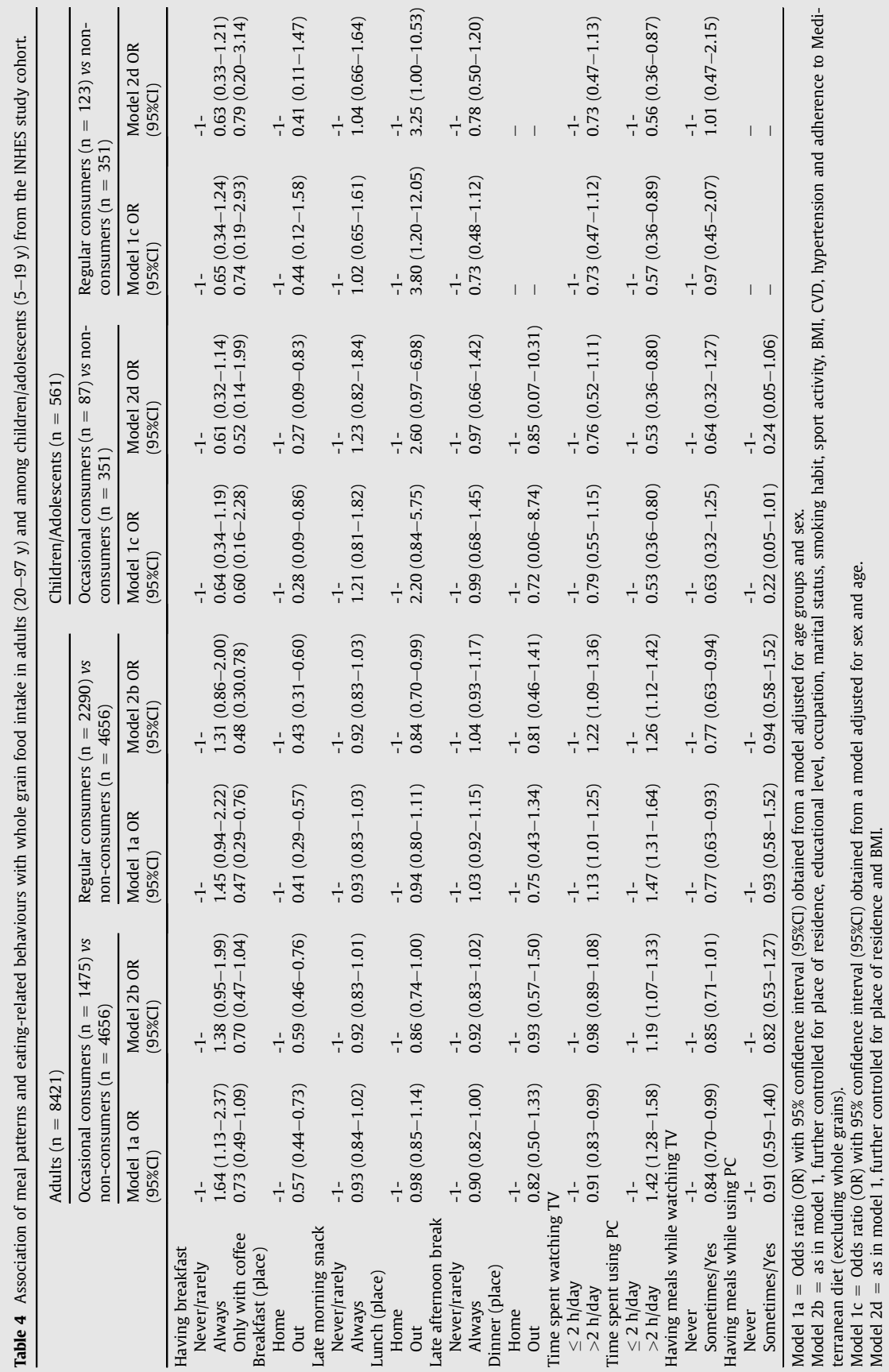


Table 5 Psychosocial factors associated with whole grain intake in adults (20-97 years)

\begin{tabular}{|c|c|c|c|c|}
\hline & \multicolumn{2}{|c|}{$\begin{array}{l}\text { Occasional consumers }(\mathrm{n}=1475) \text { vs non- } \\
\text { consumers }(\mathrm{n}=4656)\end{array}$} & \multicolumn{2}{|c|}{$\begin{array}{l}\text { Regular consumers }(\mathrm{n}=2290) \text { vs non- } \\
\text { consumers }(\mathrm{n}=4656)\end{array}$} \\
\hline & Model 1 OR (95\%CI) & Model 2 OR (95\%CI) & Model 1 OR (95\%CI) & Model 2 OR (95\%CI) \\
\hline \multicolumn{5}{|l|}{ Self-reported health status } \\
\hline Very good & $-1-$ & $-1-$ & $-1-$ & $-1-$ \\
\hline Bad & $0.47(0.41-0.54)$ & $0.51(0.45-0.59)$ & $0.41(0.36-0.46)$ & $0.44(0.38-0.51)$ \\
\hline \multicolumn{5}{|l|}{ Type of distress } \\
\hline \multicolumn{5}{|l|}{ Adverse life events } \\
\hline None & $-1-$ & $-1-$ & $-1-$ & $-1-$ \\
\hline At least one & $0.92(0.80-1.06)$ & $0.92(0.80-1.07)$ & $1.00(0.86-1.17)$ & $0.93(0.79-1.09)$ \\
\hline \multicolumn{5}{|l|}{ Occupation-related } \\
\hline Never & $-1-$ & $-1-$ & $-1-$ & $-1-$ \\
\hline Sometimes/most of the times & $1.03(0.76-1.38)$ & $0.94(0.69-1.29)$ & $0.83(0.60-1.13)$ & $0.70(0.50-0.97)$ \\
\hline Often/Always & $0.88(0.62-1.24)$ & $0.78(0.54-1.12)$ & $0.80(0.55-1.15)$ & $0.68(0.46-1.00)$ \\
\hline Not working/unascertained & $0.76(0.56-1.02)$ & $0.79(0.57-1.08)$ & $0.65(0.47-0.90)$ & $0.61(0.43-0.85)$ \\
\hline \multicolumn{5}{|l|}{ Family-related } \\
\hline Never & $-1-$ & $-1-$ & $-1-$ & $-1-$ \\
\hline Sometimes & $1.21(0.93-1.57)$ & $1.13(0.87-1.48)$ & $0.94(0.71-1.24)$ & $0.87(0.66-1.15)$ \\
\hline Most of the times & $0.78(0.60-1.02)$ & $0.77(0.59-1.00)$ & $0.69(0.52-0.92)$ & $0.69(0.52-0.91)$ \\
\hline Often/Always & $0.71(0.50-1.01)$ & $0.71(0.49-1.01)$ & $0.60(0.40-0.88)$ & $0.59(0.40-0.87)$ \\
\hline \multicolumn{5}{|l|}{ Job control } \\
\hline None/little & $-1-$ & $-1-$ & $-1-$ & $-1-$ \\
\hline Moderate -good & $0.86(0.70-1.05)$ & $0.82(0.66-1.01)$ & $0.80(0.64-1.01)$ & $0.77(0.61-0.98)$ \\
\hline Fairly good & $1.26(1.01-1.57)$ & $1.18(0.94-1.47)$ & $1.22(0.96-1.55)$ & $1.16(0.91-1.48)$ \\
\hline Not working/unascertained & $0.93(0.78-1.12)$ & $0.98(0.81-1.19)$ & $0.98(0.80-1.20)$ & $1.02(0.83-1.27)$ \\
\hline \multicolumn{5}{|l|}{ Financial circumstances-related } \\
\hline None/little & $-1-$ & $-1-$ & $-1-$ & $-1-$ \\
\hline Moderate & $0.80(0.60-1.08)$ & $0.73(0.54-0.99)$ & $0.65(0.49-0.89)$ & $0.55(0.40-0.76)$ \\
\hline A lot & $0.94(0.70-1.28)$ & $0.96(0.71-1.31)$ & $0.93(0.67-1.27)$ & $0.88(0.63-1.21)$ \\
\hline Not working/unascertained & $0.50(0.34-0.73)$ & $0.58(0.39-0.86)$ & $0.55(0.36-0.83)$ & $0.58(0.38-0.89)$ \\
\hline
\end{tabular}

Model 1 = Odds ratio (OR) with $95 \%$ confidence interval $(95 \% \mathrm{CI})$ obtained from a model adjusted for age groups and sex.

Model 2 = as in model 1, further controlled for place of residence, educational level, occupation, marital status, smoking habit, sport activity, BMI, CVD, hypertension and adherence to Mediterranean diet (excluding whole grains).

\section{Discussion}

The present study provides an updated evaluation of WG food consumption and its major demographic, socioeconomic, psychosocial and behavioural factors in a large sample of Italian adults, children and adolescents.

Findings showed that WG food is consumed on a regular basis ( $\geq 1$ time/week) by $26.9 \%$ of the whole study sample, with WG bread being the major food source of total WG food for both age groups, up to half of the total WG intake.

These data are in accordance with prior data from the INRAN-SCAI 2005-06 survey [15] showing that WG bread represents the principal food source of total WG intake in adults (46\%) while children were more likely to report a prevalent intake of WG breakfast cereals (32\%) that in our young population contributed to $15.7 \%$ of the total WG intake. Similarly, INHES adults showed low consumption of breakfast cereals $(4.8 \%)$ in line with the INRAN-SCAI adults (7\%), although reporting greater WG pasta intake over the total WG consumption ( $13.1 \%$ vs $2 \%$, respectively). Our results are also in line with data from a cohort of 1400 French adults [23] indicating that the highest contribution to total WG intake derived from WG bread (and toast) intake, although breakfast cereals contributed much more as compared to our Italian adult sample (24\%).
Previous population surveys reveal cultural differences in WG food consumption which is typically higher in Scandinavian countries [21] than in many other European countries.

In detail, the percentage of adult consumers (27.2\%) resulting from our study is lower than that documented by surveys conducted in USA [12] or UK [22] all reporting consumption above 50 percent, while it is similar to that reported in France (around 30\%) [23].

The relatively low consumption of WG foods observed in our Italian sample, which is fairly similar to that reported by the INRAN-SCAI national survey [15], appears to be in contrast with the fact that our population is supposed to be close to a traditional Mediterranean diet which definitely encourages higher intake of unrefined cereals.

Yet, in a global perspective, the comparison between countries needs to be interpreted with caution owing the different survey methods used to collect data on WG intake. Indeed, in our survey we used a food propensity questionnaire while others mainly relied on food intake recorded by dietary recalls $[24,25]$. Moreover, the differing food sources of WG (e.g. lack of ready to eat breakfast cereals in Italy) makes it difficult an actual comparison among countries.

Beyond providing an update of WG consumption in Italy, we also aimed at identifying the major determinants 
of (or variables correlated with) WG food consumption by analysing the association with a number of demographic, socioeconomic, psychosocial and behavioural factors.

A clear educational gradient emerged, with subjects with highest educational attainment being more likely to be regular WG consumers as opposed to those in the lowest educational group, in line with the INRAN-SCAI 2005-06 survey [16], and with data deriving from nonMediterranean settings [22]; we also found an association with occupation, with highest intakes reported among most advantaged social classes and smallest in the lower classes, as previously reported in a UK study [22].

The socioeconomic shape of WG food intake is in agreement with the socioeconomic gradient already observed for high quality diets, and may be accounted at least in part by the fact that higher educational level is usually associated with increased nutrition knowledge which possibly leads to healthier food choices [26].

In general, we found that regular WG consumers had a healthier lifestyle, including higher physical activity and abstention from tobacco, as already documented in other epidemiological settings [27].

Some meal patterns and eating-related behaviors were also associated with WG food intake, Individuals eating meals out of the home (i.e. breakfast or lunch) were less likely to consume WG food, thus suggesting that WG consumption is associated with a more regular eating behavior:

We also documented a relation of regular WG food intake with self-reported health status and stressful life events, in agreement with a large body of evidence indicating that diet quality is often associated with improved quality of life [28] or other indicators of mental well-being [29].

Regarding prevailing opinions on WG food, we found that about half of study participants believe WG products to be good for health, in agreement with previous results [31], and this might indicate the effectiveness of recent public campaigns to raise public awareness of the health benefits associated with WG food consumption.

On the other side, lack of knowledge on WG food is reported as being the most common obstacle by nonconsumers and this may suggest that both public health strategies and food companies should direct their efforts at increasing knowledge and awareness of WG products.

As compared to estimations provided by the previous INRAN-SCAI survey in 2005-06, WG consumers in Italy experienced a relatively modest increase from $23 \%$ to $26.9 \%$ in 2013 , thus leading to a serious re-thinking of public health strategies in order to increase the consumption of WG products in a more substantial manner at population level, especially in light of the numerous health benefits associated with WG intake [1-4].

Some useful suggestions come from the Danish health promotion campaign to increase WG consumption which included the following actions: increasing WG content of commercial food products; mass communication to improve consumers' knowledge about WG foods and their health benefits; and a certification logo on foods with a high WG content [30].
Of course, WG food promotion should be put within a wider context aimed to increase grain consumption globally, also in light of the fact that certain non-WG staple grain foods can be included in a healthy dietary pattern, provided that the dietary pattern maintains recommendations for calories, saturated fats, sodium and added sugars, as recently highlighted by the National Health and Nutrition Examination Survey (NHANES) suggesting that a variety of grain food groups (including enriched grains as ready-to-eat cereals) have the potential to increase consumption of shortfall nutrients [31].

\section{Strengths and limitations of the study}

This study has several strengths, including its large sample size and the large number of covariates used to accommodate at least in part confounding.

The considerable number of indicators used to assess meal patterns and the psychosocial dimension and their relation with dietary habits represents one major novelty of the present study.

Major limitations are represented by its cross-sectional and telephone-based design, the self-reported nature of data collection and lack of quantification of WG consumption.

An additional limitation is that, given that self-reported data were used, it is not possible to know whether the foods the participants described were actually WG containing.

Finally, we acknowledge that causality cannot be inferred due to the observational design of our study.

\section{Conclusions}

To the best of our knowledge, this study represents the most updated investigation on WG intake and its determinants in the Italian population.

Overall, the percentage of WG consumers emerging from our study (26.9\%) is similar to that reported by the last Italian survey (23\%) and appears to be still below estimations recorded in other countries of Europe, although comparisons should be made with caution.

Our study also showed that WG consumption clearly clusters with socioeconomic factors, health-related behaviors, meal patterns and with a number of psychosocial factors. Increasing knowledge on major determinants of regular WG intake may help defining more targeted public health strategies to increase the consumption of WG foods, especially in countries in which consumption is lower than recommended.

\section{Acknowledgments}

Financial support: The INHES Project was funded by Barilla SpA through the Italian Ministry of Economic Development within the frame of the ATENA program MI01_00093 New Technologies for Made in Italy (D.I. PII MI 6/3/2008). The present analyses were partially supported by the Italian Ministry of Health 2013 [M.B., Grant number GR-2013- 
02356060]. The funders had no role in study design, collection, analysis, and interpretation of data; in the writing of the manuscript and in the decision to submit the article for publication. All Authors were and are independent from funders and declare no other conflict of interest.

\section{Appendix A. Supplementary data}

Supplementary data to this article can be found online at https://doi.org/10.1016/j.numecd.2019.03.001.

\section{References}

[1] Mellen PB, Walsh TF, Herrington DM. Whole grain intake and cardiovascular disease: a meta-analysis. Nutr Metabol Cardiovasc Dis $2008 ; 18(4): 283-90$

[2] Iacoviello L, Bonaccio M, Cairella G, Catani MV, Costanzo S, D'Elia L, et al. Diet and primary prevention of stroke: systematic review and dietary recommendations by the ad hoc Working Group of the Italian Society of Human Nutrition. Nutr Metabol Cardiovasc Dis 2018;28(4):309-34

[3] Montonen J, Knekt P, Järvinen R, Aromaa A, Reunanen A. Wholegrain and fiber intake and the incidence of type 2 diabetes. Am J Clin Nutr 2003;77(3):622-9.

[4] Aune D, Chan DS, Lau R, Vieira R, Greenwood DC, Kampman E, et al. Dietary fibre, whole grains, and risk of colorectal cancer: systematic review and dose-response meta-analysis of prospective studies. BMJ 2011;10(343):d6617.

[5] Albertson AM, Reicks M, Joshi N, Gugger CK. Whole grain consumption trends and associations with body weight measures in the United States: results from the cross sectional National Health and Nutrition Examination Survey 2001-2012. Nutr J 2016;22(15):8.

[6] Tighe P, Duthie G, Vaughan N, Brittenden J, Simpson WG, Duthie S, et al. Effect of increased consumption of whole-grain foods on blood pressure and other cardiovascular risk markers in healthy middle-aged persons: a randomized controlled trial. Am J Clin Nutı 2010;92(4):733-40.

[7] Giacco R, Clemente G, Cipriano D, Luongo D, Viscovo D, Patti L, et al. Effects of the regular consumption of wholemeal wheat foods on cardiovascular risk factors in healthy people. Nutr Metab Cardiovasc Dis 2010;20(3):186-94.

[8] Ross AB, Kristensen M, Seal CJ, Jacques P, McKeown NM. Recommendations for reporting whole-grain intake in observational and intervention studies. Am J Clin Nutr 2015;101(5):903-7.

[9] Ross AB, van der Kamp JW, King R, Lê KA, Mejborn H, Seal CJ, et al. Healthgrain Forum. Perspective: a definition for whole-grain food products-recommendations from the healthgrain Forum. Adv Nutr 2017;8(4):525-31.

[10] http://www.salute.gov.it/imgs/C_17_pubblicazioni_652_allegato. pdf.

[11] Reicks M, Jonnalagadda S, Albertson AM, Joshi N. Total dietary fiber intakes in the US population are related to whole grain consumption: results from the National Health and Nutrition Examination Survey 2009 to 2010. Nutr Res 2014;34(3):226-34.

[12] McGuire S. U.S. Department of agriculture and U.S. Department of health and human services, dietary guidelines for Americans, 2010. 7th ed. Washington, DC: U.S. Government Printing Office; 2011. p. 293-4. January 2011. AdvNutr.2(3).

[13] Frølich W, Aman P, Tetens I. Whole grain foods and health - a Scandinavian perspective. Food Nutr Res 2013;57.

[14] Bach-Faig A, Berry EM, Lairon D, Reguant J, Trichopoulou A, Dernini S, et al. Mediterranean diet pyramid today. Science and cultural updates. Publ Health Nutr 2011;14(12A):2274-84.
[15] Sette S, D'Addezio L, Piccinelli R, Hopkins S, Le Donne C, Ferrari M, et al. Intakes of whole grain in an Italian sample of children, adolescents and adults. Eur J Nutr 2017;56(2):521-33.

[16] Pounis G, Bonanni A, Ruggiero E, Di Castelnuovo A, Costanzo S, Persichillo M, et al. Food group consumption in an Italian population using the updated food classification system FoodEx2: results from the Italian Nutrition \&HEalth Survey (INHES) study. Nutr Metabol Cardiovasc Dis 2017;27(4):307-28.

[17] Panagiotakos DB, Pitsavos C, Arvaniti F, Stefanadis C. Adherence to the Mediterranean food pattern predicts the prevalence of hypertension, hypercholesterolemia, diabetes and obesity, among healthy adults; the accuracy of the MedDietScore. Prev Med 2007; 44(4):335-40.

[18] Serra-Majem L, Ribas L, Ngo J, Ortega RM, García A, PérezRodrigo C, et al. Food, youth and the mediterranean diet in Spain. Development of KIDMED, mediterranean diet quality index in children and adolescents. Publ Health Nutr 2004;7(7):931-5.

[19] Rosengren A, Hawken S, Ounpuu S, Sliwa K, Zubaid M, Almahmeed WA, et al. Association of psychosocial risk factors with risk of acute myocardial infarction in 11119 cases and 13648 controls from 52 countries (the INTERHEART study): case-control study. Lancet 2004;364(9438):953-62. 11-17.

[20] Cole TJ, Bellizzi MC, Flegal KM, Dietz WH. Establishing a standard definition for child overweight and obesity worldwide: international survey. BMJ 2000;320(7244):1240-3. 6.

[21] Kyrø C, Skeie G, Dragsted LO, Christensen J, Overvad K, Hallmans G, et al. Intake of whole grain in Scandinavia: intake, sources and compliance with new national recommendations. Scand J Publ Health 2012;40(1):76-84.

[22] Mann KD, Pearce MS, McKevith B, Thielecke F, Seal CJ. Low whole grain intake in the UK: results from the National Diet and Nutrition Survey rolling programme 2008-11. Br J Nutr 2015;113(10): 1643-51. 28.

[23] Bellisle F, Hébel P, Colin J, Reyé B, Hopkins S. Consumption of whole grains in French children, adolescents and adults. Br J Nutr 2014;112(10):1674-84.

[24] Berg C, Lappas G, Wolk A, Strandhagen E, Torén K, Rosengren A, et al. Eating patterns and portion size associated with obesity in a Swedish population. Appetite 2009;52(1):21-6.

[25] Alexy U, Zorn C, Kersting M. Whole grain in children's diet: intake, food sources and trends. Eur J Clin Nutr 2010;64(7):745-51.

[26] Bonaccio M, Di Castelnuovo A, Costanzo S, De Lucia F, Olivieri M, Donati $\mathrm{MB}$, et al. Nutrition knowledge is associated with higher adherence to Mediterranean diet and lower prevalence of obesity. Results from the Moli-sani study. Appetite 2013;68: 139-469.

[27] O'Neil CE, Nicklas TA, Zanovec M, Cho S. Whole-grain consumption is associated with diet quality and nutrient intake in adults: the National Health and Nutrition Examination Survey, 19992004. J Am Diet Assoc 2010;110(10):1461-8.

[28] Bonaccio M, Di Castelnuovo A, Bonanni A, Costanzo S, De Lucia F, Pounis G, et al. Moli-sani project Investigators. Adherence to a Mediterranean diet is associated with a better health-related quality of life: a possible role of high dietary antioxidant content. BMJ Open 2013 Aug 13;3(8).

[29] Bonaccio M, Di Castelnuovo A, Costanzo S, Pounis G, Persichillo M, Cerletti C, et al. Mediterranean-type diet is associated with higher psychological resilience in a general adult population: findings from the Moli-sani study. Eur J Clin Nutr 2018;72(1):154-60.

[30] Greve C, Neess RI. The evolution of the whole grain partnership in Denmark. copenhagen business school and the Danish whole grain partnership: copenhagen. 2014. https://www.fuldkorn.dk/ media/179349/the-evolution-of-the-whole-grain-partnership-indenmark.pdf.

[31] Papanikolaou Y, Fulgoni VL. Grain foods are contributors of nutrient density for American adults and help close nutrient recommendation gaps: data from the national health and nutrition examination survey, 2009-2012. Nutrients 2017 Aug $14 ; 9(8)$. 
CHAPTER 4.

Socioeconomic and psychosocial determinants of adherence to the Mediterranean diet in a general adult Italian population

Ruggiero E, Di Castelnuovo A, Costanzo S, Persichillo M, Bracone F, Cerletti C, Donati MB, de Gaetano G, Iacoviello L, Bonaccio M; INHES Study Investigators

Eur J Public Health. 2018 Jul 17. 



\title{
Socioeconomic and psychosocial determinants of adherence to the Mediterranean diet in a general adult Italian population
}

\author{
Emilia Ruggiero ${ }^{1}$, Augusto Di Castelnuovo ${ }^{1}$, Simona Costanzo ${ }^{1}$, Mariarosaria Persichillo ${ }^{1}$, \\ Francesca Bracone ${ }^{1}$, Chiara Cerletti ${ }^{1}$, Maria Benedetta Donati ${ }^{1}$, Giovanni de Gaetano ${ }^{1}$, \\ Licia lacoviello ${ }^{1,2}$, Marialaura Bonaccio ${ }^{1}$, on behalf of the INHES Study Investigators* \\ 1 Department of Epidemiology and Prevention, IRCCS Istituto Neurologico Mediterraneo NEUROMED, 86077 Pozzilli (IS), \\ Italy \\ 2 Department of Medicine and Surgery, Research Center in Epidemiology and Preventive Medicine (EPIMED), University \\ of Insubria, 21100 Varese, Italy \\ * INHES Study Investigators are listed in the Appendix.
}

Correspondence: Licia lacoviello, Department of Epidemiology and Prevention, IRCCS Istituto Neurologico Mediterraneo NEUROMED, Via dell'Elettronica, 86077 Pozzilli (IS), Italy. Tel: +39 (0) 8659 29664, Fax: +39 (0) 8659 27575, e-mail: licia.iacoviello@moli-sani.org

\begin{abstract}
Background: To evaluate the adherence to Mediterranean diet (MD) and its major socioeconomic and psychosocial determinants in a large sample of the Italian population, covering three main geographical areas of the Country (Southern, Central and Northern). Methods: Data were obtained from the Italian Nutrition \& Health Survey (INHES), including a total of 7, 430 participants (age $>20$ ) recruited from all over Italy (2010-13). Dietary information was collected by the European Food Propensity Questionnaire. Adherence to MD was assessed by using the MedDietScore based on 11 food groups. Associations were tested by multivariable logistic regression analysis (Odds ratio [OR] with $95 \% \mathrm{Cl}$ ). Results: Adherence to MD was higher in Southern Italy as compared with the Northern $(\mathrm{OR}=1.34 ; 95 \% \mathrm{Cl} 1.18-1.53)$, and was closely associated with adult age $(\mathrm{OR}=2.40 ; 1.61-3.58$ for those aged $>75$ years as compared with $20-34$ years) and higher educational level (OR $=1.77 ; 1.40-2.24$ for post secondary education as opposed to lowest educational attainment). Subjects reporting adverse life events and those with family-related stress were less likely to show an optimal adherence to MD $(O R=0.55 ; 0.46-0.67$ and $\mathrm{OR}=0.44 ; 0.28-0.69$, for highest vs. lowest tertile, respectively) as compared with adequate controls. A number of eating behaviours were also inversely associated with MD, such as consuming higher amount of alcohol in the weekend than in week days. Conclusions: Adherence to MD is strongly determined by age, geographical area and educational level. Psychosocial factors and several eating behaviours are also closely associated.
\end{abstract}

\section{Introduction}

The Mediterranean diet (MD) is a traditional dietary pattern mainly followed in the Mediterranean regions $^{1-3}$ and is considered the golden standard of healthy nutrition, being strongly associated with decreased morbidity and mortality, especially from cardiovascular and cerebrovascular causes. ${ }^{4,5}$

More recently, a global shifting from the MD was documented especially in the Mediterranean regions that experienced the greatest loss of adherence as opposed to non-Mediterranean Countries, ${ }^{6-8}$ to such an extent that a nutrition transition issue emerged also for Mediterranean populations. ${ }^{9,10}$ Moreover, this downward trend was observed also for children and adolescents. ${ }^{11-13}$ The withdrawal from an MD could be influenced by numerous factors, and more recently a likely impact of the economic crisis has been proposed as a major cause for the shifting from this dietary pattern. ${ }^{8,14}$ In this context, improving the understanding of the major determinants associated with an $\mathrm{MD}$ is of crucial importance. Adherence to MD clusters with other healthy behaviours (i.e. physical activity and abstention from tobacco) is strongly associated with socioeconomic status, ${ }^{15}$ and age. ${ }^{16}$ The socioeconomic gradient of diet quality has been well documented mainly in Northern European and US settings, ${ }^{17,18}$ although evidence from Mediterranean regions has been recently presented. ${ }^{15}$

Most of the evidence on possible determinants associated with an MD in Italy comes from relatively small samples of subjects, often recruited in specific geographical areas that may not represent the whole Italian territory, ${ }^{16,19-22}$ while the latest dietary survey at national level was performed more than a decade ago. ${ }^{2}$

In light of the above, the main aim of this study is 2-fold: first, to evaluate the actual adherence to MD in a large sample of the Italian population recruited from all over Italy within the Italian Nutrition \& Health Survey (INHES), in order to provide updated data on the actual dietary habits of Italian people; second, to investigate major demographic, socioeconomic, psychosocial and behavioural factors possibly associated with adherence to $\mathrm{MD}$, also with the purpose of providing new evidence for targeting appropriate intervention strategies at population level.

\section{Methods}

\section{Study population}

The INHES is a 3-year telephone-based survey on nutrition and health specifically designed to collect information on the dietary habits (quality, quantity and patterns), food choice determinants and food health awareness of the Italian population according to different geographical distribution, age, gender and socioeconomic profile.

Between November 2010 and November 2013, 9319 women and men aged $\geq 5$ years from all over Italy were enrolled. Firstly, subjects $(n=9106)$ in the age range 35-79 years, recruited in the 2008-2012 wave of the Italian Cardiovascular Epidemiologic Observatory ${ }^{24,25}$ were invited to participate in the INHES survey. Once they accepted, 
participants were asked to invite one relative older than 79 or younger than 35 years to join the survey. Finally, 5385 (59.1\%) from the original population and 3754 from their relatives for a total of 9139 subjects were included in the survey. These subjects were distributed in the four seasons (excluding Christmas, Easter and mid-August periods). The survey calendar was organized to capture an adequate proportion of weekdays and weekend days at group level. The recruitment was performed using computerassisted-telephone-interviewing. A full description of the study design is reported elsewhere. ${ }^{26}$

For the purpose of this study, we included individuals aged $>20$ years $(n=8772)$ and omitted subjects with self-reported diagnosis of CVD $(3.1 \%)$, cancer $(3.2 \%)$ and diabetes $(7.1 \%)$ or missing values for major variables of interest (2.6\%). Finally, a total of 7430 subjects were included in the analyses.

\section{Dietary assessment}

Data on food intake were collected by the Italian version of the European Food Propensity Questionnaire. ${ }^{27}$ Adherence to MD was evaluated by using the MedDietScore as proposed by Panagiotakos. ${ }^{28}$ Assessment was based on the monthly consumption of eleven foods groups: cereals (bread, pasta, other grains, biscuits etc.), fruit, vegetables, legumes, potatoes, fish, meat and meat products, poultry, full fat dairy products (cheese, yoghurt and milk), olive oil and alcohol intake. Ratings (from 0 to 5 or the reverse) were assigned to each food group according to their position in the MD pyramid.

For the consumption of items presumed to be close to this pattern, a score 0 was assigned when someone reported no consumption, score 1 when they reported consumption of 1-4 servings/month, score 2 for 5-8 servings/month, score 3 for 9-12 servings/month, score 4 for 13 18 servings/month and score 5 for more than 18 servings/month. For the consumption of foods considered to be far from the MD (meat and meat products, poultry and full fat dairy products), scores on a reverse scale (i.e. 5 when someone reported no consumption to 0 when they reported almost daily consumption) were assigned. Alcohol intake was scored 5 for consumption of less than $300 \mathrm{ml}$ of alcohol/day, 0 for consumption of more than $700 \mathrm{ml} /$ day and $4-1$ for consumption of $300,400-500,600$ and 700 or $0 \mathrm{ml} /$ day, respectively. The final score potentially ranged between 0 and 55 , the latter reflecting maximal adherence to $\mathrm{MD}$. For analysis purposes, the score was also used as exact tertiles. ${ }^{28}$

\section{Meal patterns and eating behaviours}

Meal patterns comprised either frequency of main meals (breakfast, lunch, dinner) or frequency of main meals eaten out of home (breakfast, lunch and dinner). A number of eating behaviours were also investigated as patterns of alcohol consumption (regular days/week end), frequency of use of vending machine, kebab consumption, and having meals while watching TV or using PC. Information on daily amount of time spent in watching TV/using PC was also collected.

\section{Socioeconomic and psychosocial factors}

Education was based on the highest qualification attained and was categorized as up to elementary school (corresponding to $\leq 5$ years of study), lower secondary ( $>5 \leq 8$ years), upper secondary (8-13 years), post-secondary ( $>13$ years).

Present occupation was assembled into six groups: manual, nonmanual, housewife, retired, student and unemployed. Marital status was intended as married/living in a couple, single, separated/ divorced and widowed.

Self-rated health was assessed through one-item question 'In general, how would you rate your health status' and responses were arranged along a five item Likert-type scale from 'very good' to 'poor'.
Adverse life events (yes/no) during the last year included questions related to health (e.g. serious illness or death), job (e.g. retired, job loss) or interpersonal situations (e.g. divorce, problem with friend or relative).

Data on occupation or family-related stress were obtained by the question 'How often have you experienced stress in your workplace/ family?' with answers ranging from never to always. Financialrelated stress was rated as none/little, moderate and a lot. Job control was considered as none/little, moderate-good, fairy good and not working/unascertained. ${ }^{29}$

Sleep quality was assessed through the question 'How would you rate your sleep?' with two possible answers (restless or restful).

\section{Assessment of covariates}

Information on present use of lipid-lowering drugs or medications for high blood pressure were self-reported and categorized as no/yes. Body mass index (BMI) was calculated by using self-reported measurements of height and weight, calculated as $\mathrm{kg} / \mathrm{m}^{2}$ and grouped into three categories as normal $(<25)$, overweight $(\geq 25<30)$ or obese $(\geq 30)$. Sport activity was considered as a dichotomous variable (yes/no).

The population was also stratified as living in an urban or rural environment on the basis of the urbanization level as defined by the European Institute of Statistics (EUROSTAT definition) and obtained by the tool 'Atlante Statistico dei Comuni' provided by the Italian National Institute of Statistics.

\section{Statistical analysis}

Main characteristics of the study population across levels of adherence to MD were presented as numbers and percentages, while the MedDietScore (continuous) was displayed as means with SD.

Associations for categorical or continuous variables reported in table 1 were evaluated by using general linear models adjusted for age groups and sex (PROC GENMOD and PROC GLM in SAS software for categorical and continuous variables, respectively).

Multivariable odds ratios (ORs) with 95\% CIs were used to test the likelihood of being in the highest tertile of MedDietScore (highest vs. lowest, with removal of the second tertile) in association with demographic and socioeconomic factors (table 2), meal patterns and eating behaviours (table 3) or psychosocial factors (table 4).

Two logistic regression models were fitted in order to account for possible confounding. Model 1 (table 2) was adjusted for age groups and sex, multivariable models 2 (tables 2-4) were obtained by including all the variables listed in table 1 that resulted associated with the outcome with $P$ values $<0.20$.

Dummy variables for missing values were created. Two-sided $P$ values $<0.05$ was considered as statistically significant.

Subgroup analyses for geographic area were undertaken. Appropriate multiplicative terms for testing interaction of geographical area (Northern, Central and Southern regions) with main demographic and socioeconomic factors were included in the multivariable models to test for a difference of effect of geographic area in modulating the relationship between $\mathrm{MD}$ and its determinants.

The data analysis was generated using SAS/STAT software, Version 9.4 of the SAS System for Windows 2009. SAS Institute Inc. and SAS are registered trademarks of SAS Institute Inc., Cary, NC, USA.

\section{Results}

Table 1 shows the characteristics of the whole population across tertiles of adherence to MD. Mean adherence to MD was 29.6 (SD $=5.4$ ), ranging from 5 to 49 . 
Table 1 Characteristics of the population according to levels of adherence to the MD

\begin{tabular}{|c|c|c|c|c|c|}
\hline & \multirow[b]{2}{*}{ Whole sample } & \multicolumn{4}{|c|}{ Adherence to MD (MedDietScore) } \\
\hline & & First tertile & Second tertile & Third tertile & $P$ value \\
\hline MedDietScore (min-max) & $5-49$ & $5-28$ & $29-31$ & $32-49$ & - \\
\hline MedDietScore (mean, SD) & $29.6(5.4)$ & $24.4(5.4)$ & $30.0(0.8)$ & $34.1(2.3)$ & - \\
\hline$n, \%$ & 7430 & $2514(33.8)$ & $2204(29.7)$ & $2712(36.5)$ & - \\
\hline Sex (men) & 45.7 & 38.7 & 51.1 & 47.8 & $<0.0001$ \\
\hline Geographical area & & & & & 0.0009 \\
\hline Northern & 41.0 & 42.7 & 41.7 & 38.9 & \\
\hline Centre & 16.8 & 17.1 & 17.4 & 16.1 & \\
\hline Southern & 42.1 & 40.2 & 40.9 & 44.9 & \\
\hline Place of living & & & & & 0.0002 \\
\hline Rural & 13.6 & 15.3 & 13.7 & 11.9 & \\
\hline Urban & 86.4 & 84.6 & 86.3 & 88.1 & \\
\hline Age groups & & & & & $<0.0001$ \\
\hline $20-34$ & 6.2 & 8.4 & 6.0 & 4.3 & \\
\hline $35-49$ & 31.8 & 33.1 & 31.7 & 30.7 & \\
\hline $50-64$ & 33.5 & 29.8 & 33.2 & 37.3 & \\
\hline $65-74$ & 18.9 & 18.5 & 19.7 & 18.6 & \\
\hline$\geq 75$ & 9.6 & 10.1 & 9.5 & 9.1 & \\
\hline Educational level & & & & & $<0.0001$ \\
\hline Up to elementary & 15.5 & 16.9 & 16.2 & 13.6 & \\
\hline Lower secondary & 25.5 & 27.6 & 25.7 & 23.4 & \\
\hline Upper secondary & 41.7 & 40.8 & 41.4 & 42.8 & \\
\hline Post-secondary & 17.3 & 14.8 & 16.7 & 20.1 & \\
\hline Occupation & & & & & 0.20 \\
\hline Manual & 19.1 & 19.0 & 19.8 & 18.6 & \\
\hline Non-manual & 33.7 & 32.5 & 33.4 & 35.2 & \\
\hline Housewife & 11.2 & 12.0 & 10.3 & 11.0 & \\
\hline Retired & 31.8 & 31.5 & 32.3 & 31.7 & \\
\hline Student & 1.1 & 1.6 & 1.2 & 0.7 & \\
\hline Unemployed & 3.0 & 3.3 & 3.0 & 2.8 & \\
\hline Marital status & & & & & 0.10 \\
\hline Married /in couple & 75.5 & 72.7 & 76.5 & 77.1 & \\
\hline Single & 14.8 & 16.9 & 14.2 & 13.4 & \\
\hline Separated/divorced & 3.3 & 2.7 & 3.2 & 3.8 & \\
\hline Widowed & 6.4 & 7.6 & 6.0 & 5.7 & \\
\hline Smoking habit & & & & & $<0.0001$ \\
\hline No & 60.7 & 65.3 & 60.5 & 56.5 & \\
\hline Current & 16.7 & 17.7 & 16.5 & 15.9 & \\
\hline Former & 20.6 & 15.5 & 21.0 & 25.0 & \\
\hline Occasional & 2.0 & 1.4 & 2.0 & 2.6 & \\
\hline Sport activity & 19.4 & 15.9 & 18.4 & 23.4 & $<0.0001$ \\
\hline Drugs for hypertension & 25.9 & 24.8 & 26.7 & 26.3 & 0.60 \\
\hline Drugs for hyperlipidaemia & 10.0 & 9.6 & 9.8 & 10.4 & $<0.0001$ \\
\hline BMI & & & & & 0.069 \\
\hline Normal weight & 49.8 & 51.2 & 48.5 & 49.5 & \\
\hline Overweight & 37.8 & 35.1 & 39.2 & 39.2 & \\
\hline Obese & 12.4 & 13.6 & 12.4 & 11.3 & \\
\hline
\end{tabular}

Numbers are presented as percentages unless otherwise stated. $P$ values adjusted for sex and age groups.

People with the highest adherence to MD were more likely to be from Southern Italy and to have a higher educational level. Former/ occasional smokers and sport activity were also associated with an optimal adherence to MD (table 1).

The odds of being in the uppermost level of MD increased according to age groups as well as living in Southern regions or in an urban environment rather that in rural areas (table 2; multivariable model 2).

A clear educational gradient in MD emerged, with subjects with highest education reporting greater adherence to this dietary pattern, while no association with occupation was found (table 2; multivariable model 2).

People exhibiting a healthier lifestyle were also more likely to be closer to an MD diet, as those engaged in sport activities, as well as occasional/former smokers (table 2; multivariable model 2), while no association was found with BMI (table 2).

The association of MD with some demographic and socioeconomic factors was likely to be modified by geographical area; subgroup analyses (Supplementary table S1) revealed an effect modification of geographical area for the association of MD with age groups and place of living. Indeed, adult and older subjects were more likely to adhere to an MD in Central and Southern regions, rather than in the Northern part of Italy ( $\mathrm{p}$ for interaction $=0.027$ ); moreover, living in an urban environment was inversely associated with $\mathrm{MD}$ in the Northern part of Italy $(\mathrm{OR}=0.55 ; 0.38-0.79)$, and positively in the Central $(\mathrm{OR}=1.18 ; 0.76-1.85)$ and Southern regions of the country $(\mathrm{OR}=1.91 ; 1.54-2.38 ; P$ for interaction $<.0001)$.

A number of behaviours were also inversely associated with MD adhesion, as consuming relatively higher amount of alcohol in the week end as opposed to weekdays ( $\mathrm{OR}=0.61 ; 95 \% \mathrm{CI} 0.46-0.81$ for higher amounts vs. same intake; table 3 ), as well as spending more time watching TV $(\mathrm{OR}=0.45 ; 95 \%$ CI $0.33-0.60$, for $>4 \mathrm{vs} .<1 \mathrm{~h} /$ day; table 3).

Subjects usually reporting having breakfast out of home $(\mathrm{OR}=$ 0.63 ; $95 \%$ CI $0.47-0.85$ ), those using vending machine more frequently $(\mathrm{OR}=0.78 ; 95 \% \mathrm{CI} 0.61-0.99)$ or consuming kebab up to three times a month $(\mathrm{OR}=0.71 ; 95 \% \mathrm{CI} 0.61-0.84)$ were less likely to report closer adherence to MD (table 3). 
Table 2 Demographic and socioeconomic factors associated with adherence to the MD

\begin{tabular}{|c|c|c|}
\hline & \multicolumn{2}{|l|}{ MedDietScore } \\
\hline & $\begin{array}{l}\text { OR }(95 \% \mathrm{Cl}) \\
\text { Model } 1\end{array}$ & $\begin{array}{l}\text { OR }(95 \% \mathrm{Cl}) \\
\text { Model } 2\end{array}$ \\
\hline \multicolumn{3}{|l|}{ Age groups } \\
\hline $20-34$ & $-1-$ & $-1-$ \\
\hline $35-49$ & $1.79(1.40-2.30)$ & $1.74(1.29-2.33)$ \\
\hline $50-64$ & $2.43(1.90-3.10)$ & $2.44(1.79-3.33)$ \\
\hline $65-74$ & $1.97(1.52-2.55)$ & $2.36(1.65-3.40)$ \\
\hline$\geq 75$ & $1.72(1.29-2.30)$ & $2.40(1.61-3.58)$ \\
\hline \multicolumn{3}{|l|}{$\operatorname{Sex}$} \\
\hline Women & $-1-$ & $-1-$ \\
\hline Men & $1.32(1.21-1.43)$ & $1.36(1.20-1.55)$ \\
\hline \multicolumn{3}{|l|}{ Geographical area } \\
\hline Northern & $-1-$ & $-1-$ \\
\hline Central & $1.05(0.89-1.23)$ & $1.01(0.86-1.19)$ \\
\hline Southern & $1.24(1.10-1.40)$ & $1.34(1.18-1.53)$ \\
\hline \multicolumn{3}{|l|}{ Place of living } \\
\hline Rural & $-1-$ & $-1-$ \\
\hline Urban & $1.36(1.16-1.59)$ & $1.36(1.15-1.61)$ \\
\hline \multicolumn{3}{|l|}{ Educational level } \\
\hline Up to Elementary & $-1-$ & $-1-$ \\
\hline Lower secondary & $1.07(0.88-1.29)$ & $1.02(0.84-1.24)$ \\
\hline Upper secondary & $1.45(1.20-1.75)$ & $1.35(1.11-1.64)$ \\
\hline Post-secondary & $1.95(1.57-2.42)$ & $1.77(1.40-2.24)$ \\
\hline \multicolumn{3}{|l|}{ Occupation } \\
\hline Manual & $-1-$ & $-1-$ \\
\hline Non-manual & $1.20(1.02-1.40)$ & $0.96(0.81-1.15)$ \\
\hline Housewife & $1.10(0.89-1.37)$ & $1.08(0.86-1.36)$ \\
\hline Retired & $0.99(0.80-1.37)$ & $0.89(0.72-1.11)$ \\
\hline Student & $0.86(0.46-1.60)$ & $0.78(0.41-1.46)$ \\
\hline Unemployed & $1.06(0.75-1.49)$ & $0.92(0.65-1.31)$ \\
\hline \multicolumn{3}{|l|}{ Marital status } \\
\hline Married /in couple & $-1-$ & $-1-$ \\
\hline Single & $0.98(0.82-1.18)$ & $0.97(0.80-1.17)$ \\
\hline Separated /divorced & $1.37(1.00-1.88)$ & $1.33(0.96-1.84)$ \\
\hline Widowed & $0.83(0.65-1.05)$ & $0.90(0.70-1.16)$ \\
\hline \multicolumn{3}{|l|}{ Smoking habit } \\
\hline No & $-1-$ & $-1-$ \\
\hline Current & $0.98(0.84-1.14)$ & $0.99(0.84-1.16)$ \\
\hline Former & $1.62(1.40-1.88)$ & $1.59(1.37-1.85)$ \\
\hline Occasional & $2.20(1.46-3.33)$ & $2.02(1.33-3.08)$ \\
\hline \multicolumn{3}{|l|}{ Sport activity } \\
\hline No & $-1-$ & $-1-$ \\
\hline Yes & $1.72(1.49-1.99)$ & $1.62(1.39-1.88)$ \\
\hline \multicolumn{3}{|l|}{$\mathrm{BMI}$} \\
\hline Normal weight & $-1-$ & $-1-$ \\
\hline Overweight & $1.00(0.88-1.13)$ & $1.02(0.90-1.16)$ \\
\hline Obese & $0.75(0.63-0.90)$ & $0.80(0.67-0.97)$ \\
\hline
\end{tabular}

Model 1 = ORs with $95 \% \mathrm{Cls}$ obtained from a model adjusted for age groups (five categories) and sex.

Model 2 = as in model 1, further controlled for geographical area, place of living, educational level (four categories), occupation (six categories), marital status (four categories), smoking habit (four categories), sport activity, BMI (three categories), drugs for hypertension, use of lipid-lowering medications.

Reporting at least one adverse life event or family-related stress in the last year was inversely associated with $\mathrm{MD}(\mathrm{OR}=0.55 ; 0.46-0.67$ and $\mathrm{OR}=0.44 ; 0.28-0.69$, respectively; table 4$)$, while good selfrated health was positively associated (table 4). Sleep quality was not associated with adherence to the MD (table 4).

\section{Discussion}

This study aimed to assess the actual adherence to MD in a large representative sample of the Italian population and to investigate its
Table 3 Association of meal patterns and eating behaviours with adherence to the MD

\begin{tabular}{|c|c|c|c|}
\hline & \multicolumn{3}{|c|}{ MedDietScore } \\
\hline & $\%$ & $\begin{array}{l}\text { ORs }(95 \% \mathrm{Cl}) \\
\text { Model } 1\end{array}$ & $\begin{array}{l}\text { ORs }(95 \% \mathrm{Cl}) \\
\text { Model } 2\end{array}$ \\
\hline \multicolumn{4}{|l|}{ Breakfast } \\
\hline Home & 93.7 & $-1-$ & $-1-$ \\
\hline Out & 3.9 & $0.61(0.46-0.81)$ & $0.63(0.47-0.85)$ \\
\hline \multicolumn{4}{|l|}{ Having breakfast } \\
\hline Never/rarely & 1.6 & $-1-$ & $-1-$ \\
\hline Always & 88.6 & $0.93(0.61-1.43)$ & $0.88(0.56-1.37)$ \\
\hline Only with coffee & 8.9 & $0.83(0.52-1.32)$ & $0.78(0.48-1.26)$ \\
\hline \multicolumn{4}{|l|}{ Late morning snack } \\
\hline Never/rarely & 69.8 & $-1-$ & $-1-$ \\
\hline Always & 29.2 & $1.14(1.01-1.28)$ & $1.09(0.96-1.24)$ \\
\hline \multicolumn{4}{|l|}{ Lunch } \\
\hline Home & 86.9 & $-1-$ & $-1-$ \\
\hline Out & 11.8 & $0.97(0.81-1.15)$ & $0.97(0.80-1.17)$ \\
\hline \multicolumn{4}{|l|}{ Having lunch } \\
\hline Never/rarely & 0.5 & $-1-$ & $-1-$ \\
\hline Always & 98.7 & $0.99(0.45-2.20)$ & $0.84(0.37-1.92)$ \\
\hline \multicolumn{4}{|l|}{ Late afternoon break } \\
\hline Never/rarely & 74.3 & $-1-$ & $-1-$ \\
\hline Always & 24.7 & $1.04(0.91-1.18)$ & $1.01(0.89-1.15)$ \\
\hline \multicolumn{4}{|l|}{ Aperitif } \\
\hline Never & 82.9 & $-1-$ & $-1-$ \\
\hline Sometimes & 14.9 & $1.77(1.50-2.10)$ & $1.60(1.34-1.90)$ \\
\hline Always & 1.6 & $0.98(0.64-1.51)$ & $0.96(0.62-1.50)$ \\
\hline \multicolumn{4}{|l|}{ Dinner } \\
\hline Home & 97.7 & $-1-$ & $-1-$ \\
\hline Out & 0.8 & $0.77(0.42-1.43)$ & $0.83(0.44-1.55)$ \\
\hline \multicolumn{4}{|l|}{ Having dinner } \\
\hline Never/rarely & 0.4 & $-1-$ & $-1-$ \\
\hline Always & 98.7 & $1.94(0.89-4.25)$ & $1.87(0.84-4.14)$ \\
\hline \multicolumn{4}{|c|}{ Alcohol consumed in the week end as compared with the whole week } \\
\hline Same amount & 80.0 & $-1-$ & $-1-$ \\
\hline Higher amount & 6.5 & $0.60(0.46-0.79)$ & $0.61(0.46-0.81)$ \\
\hline Lower amount & 13.5 & $0.76(0.62-0.94)$ & $0.74(0.59-0.91)$ \\
\hline \multicolumn{4}{|l|}{ Vending machine use } \\
\hline Never & 78.5 & $-1-$ & $-1-$ \\
\hline Up to $2-3$ times/month & 9.8 & $0.87(0.72-1.06)$ & $0.86(0.70-1.04)$ \\
\hline $1-2$ times/week & 4.3 & $0.78(0.60-1.03)$ & $0.78(0.59-1.04)$ \\
\hline >3 times/week & 6.9 & $0.79(0.63-0.99)$ & $0.78(0.61-0.99)$ \\
\hline \multicolumn{4}{|l|}{ Kebab consumption } \\
\hline Never & 81.5 & $-1-$ & $-1-$ \\
\hline Up to 2-3 times/month & 17.0 & $0.76(0.65-0.89)$ & $0.71(0.61-0.84)$ \\
\hline$>1-2$ times/day or more & 0.5 & $1.16(0.48-2.80)$ & $0.87(0.35-2.16)$ \\
\hline \multicolumn{4}{|l|}{ Time spent watching TV } \\
\hline$<1 \mathrm{~h} /$ day & 7.2 & $-1-$ & $-1-$ \\
\hline $1-2$ h/day & 31.5 & $0.99(0.79-1.24)$ & $1.02(0.81-1.29)$ \\
\hline $2-3 \mathrm{~h} /$ day & 49.6 & $0.71(0.57-0.89)$ & $0.73(0.58-0.92)$ \\
\hline$>4$ h/day & 11.1 & $0.39(0.30-0.52)$ & $0.45(0.33-0.60)$ \\
\hline \multicolumn{4}{|l|}{ Time spent using PC } \\
\hline Never & 49.2 & $-1-$ & $-1-$ \\
\hline$<1 \mathrm{~h} /$ day & 20.0 & $1.40(1.19-1.65)$ & $1.17(0.98-1.39)$ \\
\hline $1-2$ h/day & 22.5 & $0.90(0.77-1.05)$ & $0.75(0.63-0.89)$ \\
\hline$>2 \mathrm{~h} /$ day & 7.6 & $1.09(0.87-1.38)$ & $0.82(0.64-1.06)$ \\
\hline \multicolumn{4}{|c|}{ Having meals while watching TV } \\
\hline Never & 92.4 & $-1-$ & $-1-$ \\
\hline Sometimes & 6.2 & $0.93(0.74-1.17)$ & $0.86(0.68-1.09)$ \\
\hline Yes & 0.9 & $0.95(0.54-1.68)$ & $1.00(0.56-1.80)$ \\
\hline Having meals while using & & & \\
\hline Never & 98.1 & $-1-$ & $-1-$ \\
\hline Sometimes & 0.9 & $0.85(0.50-1.45)$ & $0.68(0.39-1.19)$ \\
\hline Yes & 0.2 & $0.81(0.22-3.00)$ & $0.82(0.22-3.09)$ \\
\hline
\end{tabular}

Model $1=$ ORs with $95 \% \mathrm{Cls}$ obtained from a model adjusted for age groups and sex.

Model 2 = as in model 1, further controlled for geographical area, place of living, educational level, occupation, marital status, smoking habit, sport activity, BMI, drugs for hypertension, use of lipid-lowering medications. 
Table 4 Psychosocial factors associated with adherence to the MD

\begin{tabular}{|c|c|c|c|}
\hline & \multicolumn{3}{|c|}{ MedDietScore } \\
\hline & $\%$ & $\begin{array}{l}\text { OR }(95 \% \mathrm{Cl}) \\
\text { Model } 1\end{array}$ & $\begin{array}{l}\text { OR }(95 \% \mathrm{Cl}) \\
\text { Model } 2\end{array}$ \\
\hline \multicolumn{4}{|l|}{ Self-reported health status } \\
\hline Very good & 16.4 & $-1-$ & $-1-$ \\
\hline Good & 67.8 & $1.58(1.35-1.85)$ & $1.63(1.38-1.92)$ \\
\hline Fair & 14.7 & $1.55(1.26-1.91)$ & $1.61(1.30-2.00)$ \\
\hline Poor & 0.5 & $0.26(0.10-0.69)$ & $0.28(0.10-0.75)$ \\
\hline \multicolumn{4}{|l|}{ Type of distress } \\
\hline \multicolumn{4}{|l|}{ Adverse life events } \\
\hline None & 90.2 & $-1-$ & $-1-$ \\
\hline At least one & 9.8 & $0.54(0.45-0.65)$ & $0.55(0.46-0.67)$ \\
\hline \multicolumn{4}{|l|}{ Occupation-related } \\
\hline Never & 2.3 & $-1-$ & $-1-$ \\
\hline Sometimes/most of the times & 48.9 & $0.86(0.59-1.26)$ & $0.95(0.64-1.41)$ \\
\hline Often/Always & 6.1 & $0.85(0.55-1.31)$ & $0.85(0.54-1.33)$ \\
\hline Not working/unascertained & 42.7 & $0.80(0.55-1.17)$ & $0.92(0.61-1.37)$ \\
\hline \multicolumn{4}{|l|}{ Family-related } \\
\hline Never & 3.0 & $-1-$ & $-1-$ \\
\hline Sometimes & 55.9 & $0.75(0.55-1.04)$ & $0.83(0.60-1.15)$ \\
\hline Most of the times & 37.5 & $0.79(0.57-1.10)$ & $0.82(0.59-1.14)$ \\
\hline Often/Always & 3.4 & $0.44(0.29-0.69)$ & $0.44(0.28-0.69)$ \\
\hline Unascertained & 0.2 & $0.29(0.07-1.17)$ & $0.31(0.07-1.33)$ \\
\hline \multicolumn{4}{|l|}{ Job control } \\
\hline None & 6.7 & $-1-$ & $-1-$ \\
\hline Moderate/Good & 15.1 & $0.71(0.54-0.92)$ & $0.75(0.58-0.99)$ \\
\hline Fairy Good & 10.2 & $0.90(0.68-1.19)$ & $0.92(0.69-1.23)$ \\
\hline Not working/unascertained & 67.9 & $0.62(0.49-0.78)$ & $0.67(0.53-0.86)$ \\
\hline \multicolumn{4}{|l|}{ Financial circumstances-related } \\
\hline None & 2.3 & $-1-$ & $-1-$ \\
\hline Moderate & 56.8 & $1.01(0.69-1.49)$ & $1.16(0.79-1.71)$ \\
\hline A lot & 37.4 & $0.68(0.46-1.00)$ & $0.84(0.57-1.24)$ \\
\hline Not working/unascertained & 3.5 & $0.53(0.33-0.87)$ & $0.63(0.38-1.04)$ \\
\hline \multicolumn{4}{|l|}{ Type of sleep } \\
\hline Restful & 89.0 & $-1-$ & $-1-$ \\
\hline Restless & 8.9 & $1.01(0.84-1.22)$ & $1.02(0.84-1.24)$ \\
\hline
\end{tabular}

Model $1=$ ORs with $95 \%$ Cls obtained from a model adjusted for age groups and sex.

Model 2 = as in model 1, further controlled for geographical area, place of living, educational level, occupation, marital status, smoking habit, sport activity, BMI, drugs for hypertension, use of lipid-lowering medications.

major demographic, socioeconomic and psychosocial determinants. So far, data on dietary habits in the Italian population were mainly obtained from relatively small samples of subjects or pertaining to specific districts, ${ }^{16,19,20}$ which can likely prevent from an actual estimation of the dietary habits across the whole country, with little chance to offer insights on possible differences at national level.

In light of this, the present results from the INHES study represent the first attempt to identify the major determinants of the MD all over Italy, in recent years, after the beginning of the economic crisis, which has been previously associated with a dramatic drop in the adherence to the MD. ${ }^{31}$

We found that men, middle-aged subjects (50-64 years) and those living in Southern Italian regions showed a better adherence to MD, in agreement with a previous Italian investigation. ${ }^{19}$

A clear educational gradient emerged, with highest educational attainment being more likely associated with MD as opposed to the lowest educational group, in line with recent evidence from the Moli-sani study where MD follows a marked socioeconomic profile. $^{15}$

However, we found an effect modification of geographical area for the association of MD with its determinants, which suggests a different contribution of some demographic and socioeconomic factors in determining the adherence to the dietary pattern. Of interest, older age resulted as a major determinant of MD in Southern parts of the country, while it seemed to play no role in the Northern regions. Such findings indicate that adherence to MD seems to be strongly affected by socio-cultural background of Italian individuals, in the southern regions as part of the population cultural heritage and affordability of prices of the goods, while in the northern regions as a health-conscious choice to which only the richer part of the population has access.

Overall, we found that urban living is associated with closer adherence to MD; however, this is true only in southern regions, while an inverse association was found in the Northern area; other studies have found that the urban environment was associated with MD in adults ${ }^{8}$ just before the recession started (2005-06), while others found that adolescents from urban areas were less likely to adopt an $\mathrm{MD},{ }^{32}$ as well as elderly individuals from Mediterranean islands. $^{33}$

An optimal MD adherence clustered with other healthy behaviours, such as being physically active, as already documented also in non-Mediterranean settings, ${ }^{27,28}$ or with being former smokers possibly because these subjects are more likely to make positive decisions concerning their health; ${ }^{34}$ yet, also occasional smokers were more likely to adopt an MD-style diet.

We failed to find an association of BMI with MD, in line with previous data showing that $\mathrm{MD}$ is unrelated to BMI also in Mediterranean populations. ${ }^{35,36}$

One major strength of this study is represented by the number of indicators used to assess the psychosocial dimension and its relationship with diet.

Our results showed that a better self-reported health status is positively associated with greater adherence to $\mathrm{MD}$, in line with previous findings. ${ }^{37,38} \mathrm{We}$ also tested the association with adverse life events, an indicator which has been poorly used to date in association with diet, and with eating disorders. ${ }^{39}$ We found that experiencing adverse life events was inversely associated with MD, net of other possible determinants possibly influencing either dietary behaviours or psychological status. More frequent family stress was also inversely related to $\mathrm{MD}$, in line with studies showing that stress and emotions are closely associated with food intake, since subjects experiencing stressful situations might try to make themselves feel better by eating or drinking. ${ }^{40}$

Finally, sleep quality was not associated with MD, and this appears in contrast with previous studies showing a positive relationship between quality of sleep or sleep duration with adherence to MD. ${ }^{41,42}$

Among eating-related behaviours, consuming different amount of alcohol in the week end as compared with week days was found inversely associated with MD suggesting that drinking regularly and in moderation is still a key feature of the Mediterranean populations. Also frequent use of vending machine is negatively associated with $\mathrm{MD}$ and this is not surprising since vending machines generally include unhealthy foods and soft drinks that are not part of a Mediterranean way of eating. Skipping meals is a predictor of poorer health outcomes, ${ }^{43}$ while the number of meals eaten out of home is a potential determinant of increase of body weight. $^{16,44}$

No association between MD and meal patterns was found, with the exception of consuming breakfast away from home, which was associated with lower likelihood of adherence to MD.

Spending much time watching TV likely acts as a proxy of sedentary behaviours and has been traditionally linked to unhealthy dietary lifestyle; in our population adherence to MD was likely to decline as time spent watching TV increased, in accordance with previous evidence evaluating this association ${ }^{45}$; similar trends were observed for time spent using PC.

\section{Strengths and limitations of the study}

This study has several strengths which include a large sample size and the large number of covariates used to accommodate at least in part confounding. 
However, our findings should be interpreted in light of several limitations. First, it is a telephone-based survey sharing all the limitations inherent to this study type, including, among others, interviewer bias and inability to use visual help. Yet, information was collected by trained interviewers; moreover, each participant received by mail a short photographs atlas and guidance notes to estimate portion sizes.

A further weakness is the decreased use of land-lines that could result in an under-representation of respondents (with younger and middle-aged persons more likely to have mobile telephones only).

Second, the cross-sectional design imposes limitations for causal inference; third, residual confounding by unmeasured factors (i.e. other psychosocial factors) cannot be fully ruled out; fourth, data (including anthropometric information) are self-reported; fifth, actual variables used in this survey cannot allow to fully describe the occupational/economic status of the participants, as only education and type of occupation were collected (there are no information about the previous job of retired individuals or data on income).

Finally, given the selected nature of study participants generalizability of the present findings should be made with caution.

\section{Conclusions}

To the best of our knowledge, this study represents the most updated investigation on MD and its determinants at the national Italian level.

Our findings confirm that an optimal adherence to MD is closely associated with middle or older age, site of residence (Southern Italy) and higher education level, in line with previous investigations from Mediterranean settings. We also found that a number of psychosocial factors are associated with MD, along with some meal patterns and eating behaviours.

Diet quality is one of the most effective lifestyle in reducing disease/mortality risk at population level ${ }^{1}$; with rates of adherence being decreased over time, especially in Mediterranean countries ${ }^{6}$ public health policies are challenged to identify the most vulnerable groups at higher risk of losing such protecting factor, also to prevent new diet-related socioeconomic disparities. ${ }^{10}$

In light of the above, improving the understanding of main determinants of MD adherence during the 21st century is of crucial importance in order to develop effective public health strategies for prevention of major chronic diseases, especially at a time of financial crisis during which efforts and resources should be strategically delivered.

\section{Supplementary data}

Supplementary data are available at EURPUB online.

\section{Funding}

The INHES Project was funded by Barilla SpA through the Italian Ministry of Economic Development within the frame of the ATENA programme MI01_00093 - New Technologies for Made in Italy (D.I. PII MI 6/3/2008). The present analyses were partially supported by the Italian Ministry of Health 2013 [M.B., Grant number GR-201302356060]. The funders had no role in study design, collection, analysis and interpretation of data; in the writing of manuscripts and in the decision to submit the article for publication. All Authors were and are independent from funders. Marialaura Bonaccio was supported by a Fondazione Umberto Veronesi Fellowship. Simona Costanzo was the recipient of a Fondazione Umberto Veronesi Travel Grant.

Conflicts of interest: None declared.

\section{Ethical standards disclosure}

This study was conducted according to the guidelines laid down in the Declaration of Helsinki and all procedures involving human subjects were approved by the Ethical Committee of the Catholic University of Rome. Verbal informed consent was obtained from all subjects. Verbal consent was witnessed and formally recorded.

\section{Key points}

- A global shifting from the Mediterranean diet (MD) was documented in the last decades especially in the Mediterranean regions.

- Evidence on determinants of MD in Italy comes from relatively small population cohorts.

- Socioeconomic and psychosocial factors are major determinants of MD.

- The understanding of main determinants of MD is crucial for developing effective public health strategies for prevention of major chronic diseases.

\section{References}

1 Willett WC, Sacks F, Trichopoulou A, et al. Mediterranean diet pyramid: a cultural model for healthy eating. Am J Clin Nutr 1995;61(Suppl 6):1402S-6S.

2 Bonaccio M, Iacoviello L, de Gaetano G. on behalf of the Moli-sani Investigators. The Mediterranean diet: the reasons for a success. Thromb Res 2012;129:401-4.

3 Trichopoulou A, Costacou T, Bamia C, Trichopoulos D. Adherence to a Mediterranean diet and survival in a Greek population. N Engl J Med 2003;348:2599-608.

4 Grosso G, Marventano S, Yang J, et al. A comprehensive meta-analysis on evidence of Mediterranean diet and cardiovascular disease: are individual components equal? Crit Rev Food Sci Nutr 2017;57:3218-32.

5 Bonaccio M, Di Castelnuovo A, Costanzo S, et al. Adherence to the traditional Mediterranean diet and mortality in subjects with diabetes. Prospective results from the MOLI-SANI study. Eur J Prev Cardiol 2016;23:400-7.

6 da Silva R, Bach-Faig A, Raidó Quintana B, et al. Worldwide variation of adherence to the Mediterranean diet, in 1961-1965 and 2000-2003. Public Health Nutr 2009;12:1676-84.

7 León-Muñoz LM, Guallar-Castillón P, Graciani A, et al. Adherence to the Mediterranean diet pattern has declined in Spanish adults. J Nutr 2012;142:1843-50.

8 Bonaccio M, Di Castelnuovo A, Bonanni A, et al. Decline of the Mediterranean diet at a time of economic crisis. Results from the Moli-sani study. Nutr Metab Cardiovas Dis 2014;24:853-60.

9 CIHEAM/FAO. Mediterranean food consumption patterns: diet, environment, society, economy and health. In: A White Paper Priority 5 of Feeding Knowledge Programme, Expo Milan 2015. Rome: CIHEAM-IAMB, Bari/FAO, 2015.

10 Bonaccio M, Bes-Rastrollo M, de Gaetano G, Iacoviello L. Challenges to the Mediterranean diet at a time of economic crisis. Nutr Metab Cardiovasc Dis 2016;26:1057-63.

11 García Cabrera S, Herrera Fernández N, et al. Kidmed test; prevalence of low adherence to the Mediterranean diet in children and young; a systematic review. Nutr Hosp 2015;32:2390-9.

12 Iaccarino Idelson P, Scalfi L, Valerio G. Adherence to the Mediterranean diet in children and adolescents: a systematic review. Nutr Metab Cardiovasc Dis 2017;27:283-99.

13 Grosso G, Galvano F. Mediterranean diet adherence in children and adolescents in southern European countries. NFS Journal 2016;3:13-19.

14 Foscolou A, Tyrovolas S, Soulis G, et al. The Impact of the financial crisis on lifestyle health determinants among older adults living in the mediterranean region: the multinational MEDIS study (2005-2015). J Prev Med Public Health 2017;50:1-9.

15 Bonaccio M, Bonanni AE, Di Castelnuovo A, et al. Low income is associated with poor adherence to a Mediterranean diet and a higher prevalence of obesity: crosssectional results from the Moli-sani study. BMJ Open 2012;2:e01685. 
16 Grosso G, Marventano S, Giorgianni G, et al. Mediterranean diet adherence rates in Sicily, southern Italy. Public Health Nutr 2014;17:2001-9.

17 Monsivais P, Aggarwal A, Drewnowski A. Are socio-economic disparities in diet quality explained by diet cost? J Epidemiol Communtiy Health 2012;66:530-5.

18 Aggarwal A, Monsivais P, Cook AJ, Drewnowski A. Does diet cost mediate the relation between socioeconomic position and diet quality? Eur J Clin Nutr 2011;65:1059-66.

19 Pelucchi C, Galeone C, Negri E, La Vecchia C. Trends in adherence to the Mediterranean diet in an Italian population between 1991 and 2006. Eur J Clin Nutr 2010;64:1052-6.

20 Leone A, Battezzati A, De Amicis R, et al. Trends of Adherence to the Mediterranean Dietary Pattern in Northern Italy from 2010 to 2016. Nutrients 2017;9:734.

21 Sofi F, Vecchio S, Giuliani G, et al. Dietary habits, lifestyle and cardiovascular risk factors in a clinically healthy Italian population: the 'Florence' diet is not Mediterranean. Eur J Clin Nutr 2005;59:584-91.

22 Marventano S, Godos J, Platania A, et al. Mediterranean diet adherence in the Mediterranean healthy eating, aging and lifestyle (MEAL) study cohort. Int $J$ Food Sci Nutr 2017;31:1-8.

23 Leclercq C, Arcella D, Piccinelli R, et al. The Italian National Food Consumption Survey INRAN-SCAI 2005-06: main results in terms of food consumption. Public Health Nutr 2009;12:2504-32.

24 Giampaoli S, Palmieri L, Donfrancesco C, et al. Osservatorio Epidemiologico Cardiovascolare/Health Examination Survey Research Group. Cardiovascular health in Italy. Ten-year surveillance of cardiovascular diseases and risk factors: osservatorio Epidemiologico Cardiovascolare/Health Examination Survey 1998e2012. Eur J Prev Cardiol 2015;22:9.

25 Laurendi G, Donfrancesco C, Palmieri L, et al. Osservatorio Epidemiologico Cardiovascolare/Health Examination Survey 2008-2012. Association of lifestyle and cardiovascular risk factors with lung function in a sample of the adult Italian population: a cross-sectional survey. Respiration 2015;89:33.

26 Pounis G, Bonanni A, Ruggiero E, et al. Food group consumption in an Italian population using the updated food classification system FoodEx2: results from the Italian Nutrition \& HEalth Survey (INHES) study. Nutr Metab Cardiovasc Dis 2017;27:307-28.

27 Illner AK, Harttig U, Tognon G, et al. Feasibility of innovative dietary assessment in epidemiological studies using the approach of combining different assessment in struments. Public Health Nutr 2011;14:1055-63.

28 Panagiotakos DB, Pitsavos C, Arvaniti F, Stefanadis C. Adherence to the Mediterranean food pattern predicts the prevalence of hypertension, hypercholesterolemia, diabetes and obesity, among healthy adults; the accuracy of the MedDietScore. Prev Med 2007;44:335-40.

29 Rosengren A, Hawken S, Ounpuu S, et al. Association of psychosocial risk factors with risk of acute myocardial infarction in 11119 cases and 13648 controls from 52 countries (the INTERHEART study): case-control study. Lancet 2004;364:953-62.

30 ISTAT: Istituto Nazionale di Statistica, Atlante statistico dei comuni. Edizione 2014 Available at: https://www.istat.it/it/archivio/113712 (15 May 2018, last date accessed).

31 Bonaccio M, Di Castelnuovo A, Bonanni A, et al. Decline of the Mediterranean diet at a time of economic crisis. Results from the Moli-sani study. Nutr Metab Cardiovasc Dis 2014;24:853-60.

32 Grosso G, Marventano S, Buscemi S, et al. Factors associated with adherence to the Mediterranean diet among adolescents living in Sicily, Southern Italy. Nutrients 2013;5:4908-23.

33 Katsarou A, Tyrovolas S, Psaltopoulou T, et al. Socio-economic status, place of residence and dietary habits among the elderly: the Mediterranean islands study. Public Health Nutr 2010;13:1614-21.

34 Boyle RG, O'Connor P, Pronk N, Tan A. Health behaviors of smokers, ex-smokers, and never smokers in an HMO. Prev Med 2000;31:177-82.

35 Buckland G, González CA, Agudo A, et al. Adherence to the Mediterranean diet and risk of coronary heart disease in the Spanish EPIC Cohort Study. Am J Epidemiol 2009;170:1518-29.

36 Sánchez-Villegas A, Henríquez-Sánchez P, Ruiz-Canela M, et al. A longitudinal analysis of diet quality scores and the risk of incident depression in the SUN Project. BMC Med 2015;13:197.

37 Bonaccio M, Di Castelnuovo A, Bonanni A, et al. Adherence to a Mediterranean diet is associated with a better health-related quality of life: a possible role of high dietary antioxidant content. BMJ Open 2013;3:13.
38 Muñoz MA, Fíto M, Marrugat J, REGICOR and HERMES investigators, et al. Adherence to the Mediterranean diet is associated with better mental and physical health. Br J Nutr 2009;101:1821-7.

39 Loth K, van den Berg P, Eisenberg ME, Neumark-Sztainer D. Stressful life events and disordered eating behaviors: findings from Project EAT. J Adolesc Health 2008;43:514-6.

40 Laitinen J, Ek E, Sovio U. Stress-related eating and drinking behavior and body mass index and predictors of this behavior. Prev Med 2002;34:29-39.

41 Campanini MZ, Guallar-Castillón P, Rodríguez-Artalejo F, et al. Mediterranean diet and changes in sleep duration and indicators of sleep quality in older adults. Sleep 2017;40.

42 Mamalaki E, Anastasiou C, Kosmidis M, et al. Associations between the Mediterranean diet and sleep in the elderly. Clin Nutr ESPEN 2018;24:185.

43 Berg C, Lappas G, Wolk A, et al. Eating patterns and portion size associated with obesity in a Swedish population. Appetite 2009;52:21-6.

44 Yao M, McCrory MA, Ma G, et al. Relative influence of diet and physical activity on body composition in urban Chinese adults. Am J Clin Nutr 2003;77:1409-16.

45 Noale M, Nardi M, Limongi F, et al. Adolescents in southern regions of Italy adhere to the Mediterranean diet more than those in the northern regions. Nutr Res $2014 ; 34: 771-9$

\section{Appendix}

\section{INHES Study Investigators}

\section{Principal Investigator}

Licia Iacoviello (Neuromed, Pozzilli and Università dell'Insubria, Varese, Italy).

\section{Study coordinator}

Americo Bonanni (Neuromed, Pozzilli, Italy).

\section{Scientific Committee}

Marialaura Bonaccio (Neuromed, Pozzilli, Italy), Americo Bonanni (Neuromed, Pozzilli, Italy), Chiara Cerletti (Neuromed, Pozzilli, Italy), Simona Costanzo (Neuromed, Pozzilli, Italy), Giovanni de Gaetano (Neuromed, Pozzilli, Italy), Augusto Di Castelnuovo (Neuromed, Pozzilli, Italy), Maria Benedetta Donati (Neuromed, Pozzilli, Italy), Licia Iacoviello (Neuromed, Pozzilli and Università dell'Insubria, Varese, Italy), Mariarosaria Persichillo (Neuromed, Pozzilli, Italy).

\section{Dietary questionnaire validation}

Mariarosaria Persichillo (Neuromed, Pozzilli, Italy) and Francesco Zito (Cardarelli Hospital, Campobasso, Italy).

\section{Questionnaire administration}

Lucia Aurisano (Epicomed Research, Campobasso, Italy), Paola Barisciano (Epicomed Research, Campobasso, Italy), Valentina Bonaccio (Epicomed Research, Campobasso, Italy), Francesca Bracone (Neuromed, Pozzilli, Italy), Francesca D Aversa (Epicomed Research, Campobasso, Italy), Francesca De Lucia (Cuore Sano Onlus, Campobasso, Italy), Giovanna Galuppo (Epicomed Research, Campobasso, Italy), Teresa Panzera (Epicomed Research, Campobasso, Italy), Filippo Petrucci (Epicomed Research, Campobasso, Italy), Anna Sciarretta (Epicomed Research, Campobasso, Italy), Angelita Verna (Epicomed Research, Campobasso, Italy).

\section{Data management and analysis}

Simona Costanzo (Neuromed, Pozzilli, Italy), Augusto Di Castelnuovo (Neuromed, Pozzilli, Italy), Marco Olivieri (Università del Molise, Campobasso, Italy). 



\section{CHAPTER 5.}

Fish intake is associated with lower cardiovascular risk in a Mediterranean population: prospective results from the Moli-sani study

Bonaccio M, Ruggiero E, Di Castelnuovo A, Costanzo S, Persichillo M, De Curtis A, Cerletti C, Donati MB, de Gaetano G, Iacoviello L, on behalf of the Moli-sani study

Nutr Metab Cardiovasc Dis. 2017 Oct;27(10):865-873. 



\title{
Fish intake is associated with lower cardiovascular risk in a Mediterranean population: Prospective results from the Moli-sani study
}

\author{
M. Bonaccio a,*, E. Ruggiero a , A. Di Castelnuovo ${ }^{\text {a }}$, S. Costanzo ${ }^{a, 1}$, M. Persichillo a \\ A. De Curtis ${ }^{\text {a }}$, C. Cerletti ${ }^{a}$, M.B. Donati ${ }^{a}$, G. de Gaetano ${ }^{a}$, L. Iacoviello ${ }^{\text {a,b }}$ on behalf of \\ the Moli-sani study Investigators ${ }^{2}$
}

${ }^{a}$ Department of Epidemiology and Prevention, IRCCS Istituto Neurologico Mediterraneo NEUROMED, 86077 Pozzilli, IS, Italy

${ }^{b}$ Department of Medicine and Surgery, University of Insubria, 21100 Varese, Italy

Received 26 April 2017; received in revised form 5 July 2017; accepted 7 August 2017

Handling editor: Dr. A. Siani

Available online 23 August 2017

\section{KEYWORDS \\ Fish intake; \\ Fatty fish; \\ Cardiovascular risk; \\ Coronary heart \\ disease; \\ Cerebrovascular \\ events; \\ Stroke}

\begin{abstract}
Background and aims: Fish consumption reportedly reduces the risk of heart disease, but the evidence of cardiovascular advantages associated with fish intake within Mediterranean cohorts is limited. The aim of this study was to test the association between fish intake and risk of composite coronary heart disease (CHD) and stroke in a large population-based cohort adhering to Mediterranean Diet.

Methods and results: Prospective analysis on 20,969 subjects free from cardiovascular disease at baseline, enrolled in the Moli-sani study (2005-2010). Food intake was recorded by the Italian version of the EPIC food frequency questionnaire. Hazard ratios were calculated by using multivariable Cox-proportional hazard models. During a median follow-up of 4.3 years, a total of 352 events occurred ( $\mathrm{n}$ of $\mathrm{CHD}=287$ and $\mathrm{n}$ of stroke $=66$ ). After adjustment for a large panel of covariates, fish intake $\geq 4$ times per week was associated with $40 \%$ reduced risk of composite CHD and stroke ( $\mathrm{HR}=0.60 ; 95 \% \mathrm{Cl} 0.40-0.90)$, and with 40\% lower risk of CHD ( $\mathrm{HR}=0.60$; $95 \% \mathrm{CI} 0.38-0.94)$ as compared with subjects in the lowest category of intake ( $<2$ times/week). A similar trend of protection was found for stroke risk although results were not significant ( $\mathrm{HR}=0.62 ; 95 \% \mathrm{CI} 0.26-1.51)$. When fish types were considered, protection against the composite outcome and CHD was confined to fatty fish intake.

Conclusions: Fish intake was associated with reduced risk of composite fatal and non-fatal CHD and stroke in a general Mediterranean population. The favourable association was likely to be driven by fatty fish.

(c) 2017 The Italian Society of Diabetology, the Italian Society for the Study of Atherosclerosis, the Italian Society of Human Nutrition, and the Department of Clinical Medicine and Surgery, Federico II University. Published by Elsevier B.V. All rights reserved.
\end{abstract}

\footnotetext{
* Corresponding author.

E-mail address: marialaura.bonaccio@neuromed.it (M. Bonaccio).

${ }^{1}$ Present address: Department of Nutrition, Harvard TH Chan School of Public Health, Boston, MA 02115, United States.

${ }^{2}$ Moli-sani Study Investigators are listed in the Appendix.
} 


\section{Introduction}

Cardiovascular disease (CVD) accounts for $45 \%$ of all deaths in Europe and increased life expectancy have determined a growing number of people living longer with CVD [1].

In light of this, understanding the major lifestyle determinants possibly associated with a reduction in CVD incidence at population level is of crucial importance, and among modifiable health-related behaviours, dietary choices represent an important tool to favourably modulate the risk of CVD either in high-risk groups or within the general population $[2,3]$.

Beyond promoting a healthy dietary lifestyle overall, dietary guidelines recommend eating fish at least two times (two servings) a week [4], because of the welldocumented inverse association with risk of developing CVD supported by several meta-analyses.

A meta-analysis of 17 cohort studies showed that both low (1 serving/week) and moderate fish consumption (2-4 servings/week) were inversely associated with coronary heart disease (CHD) mortality while no additional benefits were seen at higher intakes [5]. Advantages for cerebrovascular health were supported by findings from a metaanalysis on 16 prospective studies [6] showing that even infrequent intakes of fish (once a week) were capable to reduce the risk of stroke and such data were further confirmed in the following work by Chowdhury [7] in which highest fish intake was inversely associated with cerebrovascular risk as opposed to the lowest category of consumption, and the dose-response analysis showed that an increment of two servings a week of any fish was associated with a $4 \%$ reduced risk of cerebrovascular disease.

In fish eating populations, the protective effects of fish consumption were mainly observed in association with high amounts either for stroke [8] or for CHD [9], whereas in epidemiological settings with low habitual fish intake substantial benefits were observed even from modest amounts and mainly for fatal CVD events rather than nonfatal [10].

Yet, poor evidence has been obtained on the association of fish and CVD risk within Mediterranean population settings. Available evidence dates back to men recruited in the Italian segment of the Seven Countries Study [11] for whom consumption of fatty compared with non-fatty-fish resulted in lower CHD mortality, while more recently a study on the Spanish segment of the EPIC cohort failed to find any relationship between fish intake and stroke risk [12].

The aim of the present study was then to examine the relationship between fish consumption and risk of CHD and stroke in a general Mediterranean population, recruited in the Moli-sani study.

\section{Methods}

\section{Study population}

The Moli-sani study is a large population-based cohort study that in 2005-2010 recruited 24,325 men and women aged $\geq 35$ years from the general population of the Molise region, a central-southern area of Italy [13].

We excluded individuals with a history of CVD or unascertained CVD at baseline (7.1\%), those reporting implausible energy intakes $(<800 \mathrm{kcal} /$ day in men and $500 \mathrm{kcal} /$ day in women or $>4000 \mathrm{kcal} /$ day in men and $3500 \mathrm{kcal} /$ day in women; $3.2 \%$ ), subjects with missing information on dietary habits $(0.4 \%)$, unreliable medical or dietary questionnaires ( $1 \%$ and $3.9 \%$, respectively), subjects lost to follow-up $(0.2 \%)$ or with incomplete personal data (1.7\%). The final sample was of 20,969 individuals.

The Moli-sani study complies with the Declaration of Helsinki and was approved by the ethical committee of the Catholic University in Rome, Italy. All participants provided written informed consent.

\section{Dietary information}

Food intake during the year before enrolment was ascertained by the validated Italian version of the EPIC food frequency questionnaire [14] which includes 188 food items, classified into 45 predefined food groups on the basis of similar nutrient characteristics or culinary usage.

The EPIC questionnaire includes a specific question about fish intake comprising frequency of consumption of a number of fish types.

In the present analyses fish intake was evaluated either by using fish sub-types or total fish intake.

Categories of fish were shellfish, shrimps and crustaceous, dried fish (i.e. salted codfish), canned fish and other fish not included in the previous categories (i.e. salmon, swordfish, anchovies, etc.). Total fish came up as the sum of the above-mentioned categories.

In addition, fish consumption was also divided according to percentage of fat: fish containing up to $4 \%$ fat was classified as lean (e.g. cod, plaice, sole, hake, molluscs and crustaceous), whereas fish containing $\geq 4 \%$ fat was classified as fatty (e.g. salmon, trout, herring, mackerel and canned fish products). Both total fish and fish-types were considered as intake on a weekly basis (times per week). Regarding total fish intake, frequency was categorized as up to $2,2-4$ and $\geq 4$ times per week, while fish types were categorized as never, up to 1 time and $\geq 1$ time per week.

Adherence to the Mediterranean diet was evaluated by the Mediterranean Diet Score (MDS) [15] but, in order to control analyses for dietary habits beside fish consumption, it was deprived of fish intake.

\section{Ascertainment of cases}

Primary fatal and nonfatal incident cases of CHD (unstable angina, myocardial infarction, coronary revascularization and sudden death for unspecified cardiac event) and cerebrovascular disease that occurred in the cohort during follow-up were ascertained by linkage of the study cohort to the hospital discharge files and to the regional ReNCaM registry and death certificates (ISTAT form), by using the International Classification of Diseases, ninth revision 
(ICD-9). For CHD, ICD 9 codes 410-414 and/or reperfusion procedure (ICD-9 codes 36.0-36.9) and for cerebrovascular disease, ICD9 codes $430-432,434,436-438$ or procedure codes for carotid revascularization (ICD 9 code 38.12) were considered.

Suspected CHD deaths were identified when ICD-9 codes $410-414$ or 798 and 799 were reported as the underlying cause of death or codes $250,401-405,420-429$ as the underlying cause of death, associated with codes 410-414 as a secondary cause of death.

Suspected cerebrovascular deaths were identified when ICD 9 codes $430-438$ were reported as the underlying, antecedent, or direct cause of death.

All events were validated using procedures of the AHA, WHF, ESC, CDC and NHLBI for epidemiology and clinical research studies [16].

Time to event was calculated until the date of diagnosis of CVD, or the date of death, or the date of the last contact prior to December 2011.

\section{Statistics}

Means with standard deviations (SDs) and percentages were used to describe the characteristics of the study population across categories of total fish intake. Associations for continuous and categorical variables were evaluated by using general linear models adjusted for age, sex and energy intake (kcal/d) (PROC GENMOD and PROC GLM in SAS for categorical and continuous variables, respectively).

Risk estimates for incident CHD and stroke associated with total fish and fish-types intakes were expressed as multivariable hazard ratios (HRs) with $95 \%$ confidence intervals $(95 \% \mathrm{CI})$ and calculated by using Cox proportional hazards models. Tests for violation of the proportional hazards assumption were conducted through the introduction of linear interaction between weekly fish intake (continuous) and the time variable ( $p=0.47$ ). Hazard ratios were calculated both for categories of fish intake and as a continuous exposure (for 1-time per week increment). Potential covariates were included in the multivariable models when they resulted associated with $\mathrm{p}<0.20$ with both fish intake and CVD risk. Three multivariable models were fitted. Model 1 included age, sex and energy intake; model 2 was additionally adjusted for education, smoking, drugs for diabetes, drugs for hypertension, drugs for lipids, and MDS without fish. Finally, model 3 was additionally controlled for blood glucose, LDL-cholesterol and low-grade inflammation as likely mediating pathways of the association between fish intake and the outcomes. A possible curvilinear association between the continuous measure of fatty fish intake (g/d) and CVD risk was tested via an adjusted Cox model using a restricted cubic spline.

Dummies variables for missing values were created. Two-sided P-value $<0.05$ was considered as statistically significant. The data analysis was generated using SAS/ STAT software, Version 9.4 of the SAS System for Windows ${ }^{\odot}$ 2009. SAS Institute Inc. and SAS are registered trademarks of SAS Institute Inc., Cary, NC, USA.

\section{Results}

Fish was consumed by almost all subjects of the cohort (99.5\%). Mean total fish intake in the study sample was of $44.6 \mathrm{~g} / \mathrm{d}(\mathrm{SD}=26.3)$, similar to the amount reported within other high-fish consuming Countries, such as Spain (4) and Japan (7). Mean frequency of fish intake was of 2.4 $(\mathrm{SD}=2.2)$ times per week.

Characteristics of the study population by weekly consumption of total fish are reported in Table 1. Women, younger people, and individuals with highest socioeconomic status were more likely to consume fish more frequently. Greater intake of fish was also associated with higher prevalence of smoking, leisure-time physical activity, use of drugs for diabetes, hypertension and lipidlowering medications, higher values of LDL-cholesterol and lower levels of subclinical inflammation. Frequent fish consumption was also associated with higher adherence to a Mediterranean diet statistically deprived of fish, and greater energy intake (Table 1 ).

Over a median follow up of 4.3 years $(90,886$ personyears; lower to upper quartile: $3.4-5.3 \mathrm{y}$ ), we identified a total of 352 new cardiovascular events including 287 CHD and 66 strokes.

Risk estimates for total fish intake and the composite outcome of CHD and stroke, CHD and stroke separately are reported in Table 2. As compared to low fish intake (0-1.99 times/week), high consumption of fish ( $\geq 4$ times/week) was associated with $38 \%$ lower CVD risk ( $7 \%-58 \%$; Model 2) and $9 \%$ lower risk for 1-time per week increment (HR $=0.91$; 95\%CI 0.83-0.99; Model 2). After exclusion of CVD events occurred in the first 6 months of follow-up ( $n$ of subjects $=95$; $\mathrm{n}$ of new CVD cases $=51$ ), the magnitude of the association of total fish with CHD and stroke risk was not affected (HR $=0.64 ; 95 \% \mathrm{CI} 0.42-0.99$ for the highest vs lowest category of fish intake). Subgroup analyses for gender, age classes ( $\leq 65 \mathrm{y}$ vs $>65 \mathrm{y}$ ), income and education did not reveal any difference of effect across groups ( $p$ values for interaction $>0.05$ ).

A comparable reduction was found for CHD risk $(\mathrm{HR}=0.62 ; 95 \%$ CI 0.40-0.98; Table 2; Model 2) while results for stroke showed a similar downward trend, although statistical significance did not hold $(\mathrm{HR}=0.63$; 95\%CI 0.26-1.51; Table 2; Model 2).

In Model 3 the likely mediating effect of lipids, blood glucose and low-grade inflammation was evaluated but the results remained substantially unchanged (Table 2).

Analyses for fish types are reported in Table 3. Although not statistically significant, protection from the composite $\mathrm{CHD} /$ stroke was confined to canned fish and other fish types $(\mathrm{HR}=0.85 ; 95 \% \mathrm{CI} 0.61-1.19$ and $\mathrm{HR}=0.83 ; 95 \% \mathrm{CI}$ $0.55-1.24$ for consumption $\geq 1$ time per week vs no intake). Consumption of dried fish showed a trend toward increased CVD risk, although statistical significance was not hold $(\mathrm{HR}=1.07 ; 95 \% \mathrm{CI} 0.65-1.77)$. Similar results were observed for CHD and stroke separately (Table 3).

Risks of composite $\mathrm{CHD} /$ stroke and of $\mathrm{CHD}$ were reduced by $44 \%$ and $45 \%$ in association with fatty fish intake $\geq 1$ time per week $(\mathrm{HR}=0.56 ; 95 \% \mathrm{CI} 0.36-0.87$ and 
Table 1 Characteristics of the study population by intake of fish on a weekly basis in the Moli-sani cohort.

\begin{tabular}{|c|c|c|c|c|c|}
\hline & \multirow[t]{2}{*}{ All } & \multicolumn{3}{|c|}{ Fish intake (times per week) } & \multirow[t]{2}{*}{ P value $^{a}$} \\
\hline & & $0-1.99$ & $2-3.99$ & $\geq 4$ & \\
\hline g/d of fish (Mean, SD) & $44.6(26.3)$ & $23.0(9.6)$ & $50.7(12.6)$ & $92.5(25.9)$ & - \\
\hline $\mathrm{N}(\%)$ & 20969 & $8576(40.9)$ & $9783(46.6)$ & $2610(12.5)$ & - \\
\hline Age (year) & $54.7(11.5)$ & $55.2(11.8)$ & $54.4(11.3)$ & $54.2(11.1)$ & $<0.0001$ \\
\hline Sex (men, \%) & 46.0 & 49.2 & 44.2 & 42.3 & $<0.0001$ \\
\hline Income (EUR/year, \%) & & & & & $<0.0001$ \\
\hline$<25,000$ & 35.9 & 38.1 & 35.0 & 32.0 & \\
\hline $25,000-40,000$ & 21.0 & 18.8 & 22.7 & 22.3 & \\
\hline$>40,000$ & 12.5 & 10.7 & 13.7 & 14.0 & \\
\hline Non-respondents & 30.6 & 32.5 & 28.7 & 31.8 & \\
\hline Education (\%) & & & & & $<0.0001$ \\
\hline Up to middle school & 51.1 & 55.9 & 48.5 & 45.0 & \\
\hline Secondary school & 35.6 & 32.4 & 37.6 & 38.5 & \\
\hline University or higher & 13.2 & 11.6 & 13.8 & 16.5 & \\
\hline BMI (\%) & & & & & 0.63 \\
\hline Normal $(<25)$ & 28.4 & 28.2 & 28.4 & 29.0 & \\
\hline Overweight $(\geq 25<30)$ & 42.7 & 42.5 & 42.7 & 43.1 & \\
\hline Obese $(\geq 30)$ & 28.9 & 29.3 & 28.9 & 27.9 & \\
\hline Abdominal obesity (\%) & 72.9 & 73.7 & 72.2 & 72.8 & 0.62 \\
\hline Smokers (\%) & 23.5 & 23.5 & 23.3 & 24.3 & 0.0022 \\
\hline Leisure-time PA (met-h/day) & $3.5(4.0)$ & $3.4(4.0)$ & $3.6(3.9)$ & $4.0(3.9)$ & $<0.0001$ \\
\hline Drug for diabetes (\%) & 4.1 & 4.0 & 4.1 & 4.4 & 0.025 \\
\hline Systolic BP (mmHg) & $140.0(20.4)$ & $140.4(20.5)$ & $140.1(20.4)$ & $140.2(20.4)$ & 0.69 \\
\hline Diastolic BP (mmHg) & $82.4(9.6)$ & $82.4(9.5)$ & $82.6(9.6)$ & $82.6(9.7)$ & 0.24 \\
\hline Drugs for hypertension (\%) & 24.8 & 24.9 & 24.6 & 25.4 & 0.016 \\
\hline Drugs for lipids (\%) & 5.5 & 5.1 & 5.7 & 6.2 & 0.0003 \\
\hline Cancer (\%) & 3.1 & 3.2 & 2.9 & 3.5 & 0.37 \\
\hline Aspirin use (\%) & 2.2 & 2.4 & 2.1 & 1.7 & 0.43 \\
\hline C-reactive protein $(\mathrm{mg} / \mathrm{L})$ & $2.52(3.17)$ & $2.51(3.18)$ & $2.51(3.18)$ & $2.49(3.07)$ & 0.94 \\
\hline Low-grade inflammation ${ }^{b}$ & $-0.17(5.9)$ & $-0.07(5.9)$ & $-0.25(5.9)$ & $-0.38(5.9)$ & 0.030 \\
\hline Total cholesterol (mg/dl) & $214(41)$ & $213(42)$ & $214(41)$ & $215(42)$ & 0.15 \\
\hline HDL-cholesterol & $57.8(14.8)$ & $57.3(14.9)$ & $57.3(14.8)$ & $57.6(14.7)$ & 0.69 \\
\hline LDL-cholesterol & $131(34)$ & $130(35)$ & $131(34)$ & $132(35)$ & 0.027 \\
\hline Triglycerides (mg/dl) & $129(85)$ & $131(87)$ & $130(83)$ & $128(85)$ & 0.43 \\
\hline Blood glucose (mg/dl) & $100(24)$ & $100(23)$ & $101(24)$ & $101(24)$ & 0.061 \\
\hline Insulin $(\mathrm{pmol} / \mathrm{l})$ & $59.3(41.0)$ & $59.0(41.6)$ & $60.0(42.0)$ & $59.8(35.1)$ & 0.21 \\
\hline Mediterranean diet score ${ }^{a}$ & $3.9(1.5)$ & $3.8(1.5)$ & $3.9(1.5)$ & $4.1(1.5)$ & $<0.0001$ \\
\hline Energy intake (Kcal/day; means, SD) & $2088(573)$ & $2022(571)$ & $2119(568)$ & $2193(577)$ & $<0.0001$ \\
\hline
\end{tabular}

$\mathrm{HR}=0.55 ; 95 \% \mathrm{CI} \quad 0.34-0.89$, respectively, Table 3), whereas no effect was documented for any frequency of lean fish intake.

A cubic spline was also generated for testing linear/ nonlinear association of continuous fatty fish intake $(\mathrm{g} / \mathrm{d})$ with risk of composite CHD and stroke. We found a linear inverse relationship between fatty fish consumption and the risk for the composite outcome ( $p$ value for overall association $=0.025 ; \mathrm{p}$ value for non-linear association $=0.99$; Fig. 1 ).

\section{Discussion}

Findings from the present study show an inverse association between fish intake and risk of composite CHD and stroke in a large Mediterranean population-based cohort of adult individuals apparently free from CVD at time of recruitment. Results were also confirmed for incident CHD, while risk of stroke exhibited a downward trend, though not significant, likely due to the relatively low number of events.

To the best of our knowledge, this is the first study evaluating the association between fish intake and composite cardiovascular endpoints in a general population characterized by a relatively high adherence to the Mediterranean Diet, suggesting that higher fish consumption offers an additive protection over the cardiovascular benefit of the overall Mediterranean Diet.

Observational evidence on the CVD advantages deriving from fish intake has provided convincing results in a number of population settings with different fish consumption. In fish eating populations, such as the Japanese subjects recruited within the JPHC study with a median fish intake of $78 \mathrm{~g} / \mathrm{d}$, the highest category of intake, 
Table 2 Incidence and Hazard Ratios for fatal and non-fatal CHD and stroke according to weekly consumption of fish.

\begin{tabular}{|c|c|c|c|c|c|}
\hline & \multicolumn{3}{|c|}{ Fish intake (times/week) } & & \multirow[t]{2}{*}{1 time per week increment } \\
\hline & $0-1.99$ & $2-3.99$ & $\geq 4$ & & \\
\hline g/d (Means, SD) & $23.0(9.6)$ & $50.7(12.6)$ & 92.5 (25.9) & & - \\
\hline \multirow[t]{2}{*}{$\mathrm{N}$ of subjects, \% } & $8576(40.9)$ & $9783(46.6)$ & $2610(12.5)$ & & - \\
\hline & \multicolumn{3}{|c|}{ Composite CHD/stroke $(\mathbf{n}=352)$} & \multicolumn{2}{|l|}{$P$ for trend } \\
\hline $\mathrm{N}$ of events (rate) & $174(2.0)$ & $149(1.5)$ & $29(1.1)$ & - & \\
\hline Person-years & 36,470 & 42,757 & 11,659 & - & \\
\hline Model 1 (HR, 95\%CI) & $-1-$ & $0.82(0.66-1.02)$ & $0.62(0.42-0.92)$ & 0.0080 & $0.91(0.84-0.99)$ \\
\hline Model 2 (HR, 95\%CI) & $-1-$ & $0.81(0.65-1.02)$ & $0.62(0.42-0.93)$ & 0.0084 & $0.91(0.83-0.99)$ \\
\hline Model 3 (HR, 95\%CI) & \multicolumn{3}{|c|}{ CHD $(n=287)$} & $\begin{array}{l}0.0055 \\
P \text { for trend }\end{array}$ & $0.90(0.83-0.98)$ \\
\hline $\mathrm{N}$ of events (rate) & $140(1.6)$ & $124(1.3)$ & $23(0.9)$ & - & \\
\hline Person-years & 36,518 & 42,795 & 11,665 & - & \\
\hline Model 1 (HR, 95\%CI) & $-1-$ & $0.85(0.67-1.09)$ & $0.62(0.40-0.97)$ & 0.028 & $0.91(0.83-1.00)$ \\
\hline Model 2 (HR, 95\%CI) & $-1-$ & $0.85(0.67-1.09)$ & $0.62(0.40-0.98)$ & 0.029 & $0.91(0.83-1.00)$ \\
\hline Model 3 (HR, 95\%Cl) & \multicolumn{3}{|c|}{ Stroke $(n=66)$} & $\begin{array}{l}0.020 \\
P \text { for trend }\end{array}$ & $0.90(0.82-0.99)$ \\
\hline $\mathrm{N}$ of events (rate) & $34(0.4)$ & $26(0.3)$ & $6(0.2)$ & - & \\
\hline Person-years & 36,715 & 43,021 & 11,696 & - & \\
\hline Model 1 (HR, 95\%CI) & $-1-$ & $0.71(0.42-1.19)$ & $0.63(0.26-1.50)$ & 0.15 & $0.91(0.75-1.11)$ \\
\hline Model 2 (HR, 95\%CI) & $-1-$ & $0.70(0.42-1.18)$ & $0.63(0.26-1.51)$ & 0.15 & $0.91(0.74-1.11)$ \\
\hline Model 3 (HR, 95\%CI) & $-1-$ & $0.70(0.42-1.18)$ & $0.62(0.26-1.51)$ & 0.15 & $0.91(0.74-1.11)$ \\
\hline
\end{tabular}

Model 1 adjusted for age, sex and energy intake. Model 2: as in model 1 further adjusted for education, smoking, drugs for diabetes, drugs for hypertension, drugs for lipids, MDS without fish. Model 3: as in model 2 further adjusted for blood glucose, LDL-cholesterol and low-grade inflammation.

Table 3 Association of fish type intake (times/week) with risk of CHD and stroke.

\begin{tabular}{|c|c|c|c|c|c|c|c|c|}
\hline \multirow{2}{*}{$\begin{array}{l}\text { Weekly } \\
\text { consumption }\end{array}$} & \multirow[t]{2}{*}{$\%$} & \multirow{2}{*}{$\begin{array}{l}\text { Mean daily } \\
\text { intake (g/d) }\end{array}$} & \multicolumn{2}{|c|}{ Composite CHD/stroke } & \multicolumn{2}{|l|}{ CHD } & \multicolumn{2}{|l|}{ Stroke } \\
\hline & & & $\begin{array}{l}\mathrm{N} \text { of events/ } \\
\text { subjects }\end{array}$ & $\mathrm{HR}(95 \% \mathrm{CI})$ & $\begin{array}{l}\mathrm{N} \text { of events/ } \\
\text { subjects }\end{array}$ & $\mathrm{HR}(95 \% \mathrm{CI})$ & $\begin{array}{l}\mathrm{N} \text { of events/ } \\
\text { subjects }\end{array}$ & $\mathrm{HR}(95 \% \mathrm{CI})$ \\
\hline \multicolumn{9}{|c|}{ Shellfish, shrimps and crustaceous } \\
\hline None & 12.1 & 0.0 & $55 / 2539$ & $-1-$ & $45 / 2539$ & $-1-$ & $11 / 2539$ & $-1-$ \\
\hline$<1$ & 55.8 & $6.8(4.5)$ & $200 / 11699$ & $0.99(0.72-1.36)$ & $162 / 11699$ & $0.93(0.66-1.33)$ & $38 / 11699$ & $1.13(0.55-2.30)$ \\
\hline$\geq 1$ & 32.1 & $21.8(9.8)$ & $97 / 6731$ & $1.02(0.72-1.45)$ & $80 / 6731$ & $0.95(0.65-1.41)$ & $17 / 6731$ & $1.19(0.53-2.68)$ \\
\hline \multicolumn{9}{|l|}{ Dried fish } \\
\hline None & 11.3 & 0.0 & $28 / 2366$ & $-1-$ & $24 / 2366$ & $-1-$ & $4 / 2366$ & $-1-$ \\
\hline$<1$ & 80.2 & $5.4(4.3)$ & $287 / 16816$ & $1.03(0.69-1.53)$ & $235 / 16816$ & $0.97(0.63-1.49)$ & $53 / 16816$ & $1.44(0.51-4.06)$ \\
\hline$\geq 1$ & 8.5 & $25.7(10.6)$ & $37 / 1787$ & $1.07(0.65-1.77)$ & $28 / 1787$ & $0.95(0.55-1.67)$ & $9 / 1787$ & $1.82(0.55-6.05)$ \\
\hline \multicolumn{9}{|l|}{ Canned fish } \\
\hline None & 12.3 & 0.0 & $56 / 2582$ & $-1-$ & $42 / 2582$ & $-1-$ & $14 / 2582$ & $-1-$ \\
\hline$<1$ & 54.9 & $4.5(2.3)$ & $203 / 11516$ & $0.93(0.69-1.25)$ & $170 / 11516$ & $1.03(0.73-1.45)$ & $33 / 11516$ & $0.65(0.34-1.22)$ \\
\hline$\geq 1$ & 32.8 & $14.5(6.9)$ & $93 / 6871$ & $0.85(0.61-1.19)$ & $75 / 6871$ & $0.91(0.62-1.33)$ & $19 / 6871$ & $0.76(0.38-1.54)$ \\
\hline \multicolumn{9}{|l|}{ Other fish } \\
\hline None & 8.6 & 0.0 & $31 / 1801$ & $-1-$ & $25 / 1801$ & $-1-$ & $6 / 1801$ & $-1-$ \\
\hline$<1$ & 42.2 & $10.6(6.0)$ & $171 / 8757$ & $1.09(0.73-1.62)$ & $136 / 8757$ & $1.06(0.68-1.65)$ & $36 / 8757$ & $1.30(0.53-3.20)$ \\
\hline$\geq 1$ & 49.2 & $31.6(16.4)$ & $150 / 10311$ & $0.83(0.55-1.24)$ & $126 / 10311$ & $0.88(0.56-1.37)$ & $24 / 10311$ & $0.67(0.26-1.70)$ \\
\hline \multicolumn{9}{|l|}{ Lean fish } \\
\hline None & 3.6 & 0.0 & $14 / 745$ & $-1-$ & $10 / 745$ & $-1-$ & $4 / 745$ & $-1-$ \\
\hline$<1$ & 50.1 & $10.3(5.1)$ & $196 / 10511$ & $1.38(0.78-2.45)$ & $163 / 10511$ & $1.58(0.81-3.08)$ & $34 / 10511$ & $0.92(0.30-2.77)$ \\
\hline$\geq 1$ & 46.3 & $30.9(14.3)$ & $142 / 9713$ & $1.25(0.70-2.23)$ & $114 / 9713$ & $1.38(0.70-2.71)$ & $28 / 9713$ & $0.91(0.30-2.75)$ \\
\hline \multicolumn{9}{|l|}{ Fatty fish } \\
\hline None & 4.1 & 0.0 & $27 / 873$ & $-1-$ & $22 / 873$ & $-1-$ & $5 / 873$ & $-1-$ \\
\hline$<1$ & 52.9 & $8.9(4.8)$ & $204 / 11086$ & $0.66(0.43-1.01)$ & $164 / 11086$ & $0.62(0.39-0.99)$ & $40 / 11086$ & $0.97(0.36-2.60)$ \\
\hline$\geq 1$ & 43.0 & $24.2(11.1)$ & $121 / 9010$ & $0.56(0.36-0.87)$ & $101 / 9010$ & $0.55(0.34-0.89)$ & $21 / 9010$ & $0.69(0.24-1.94)$ \\
\hline
\end{tabular}




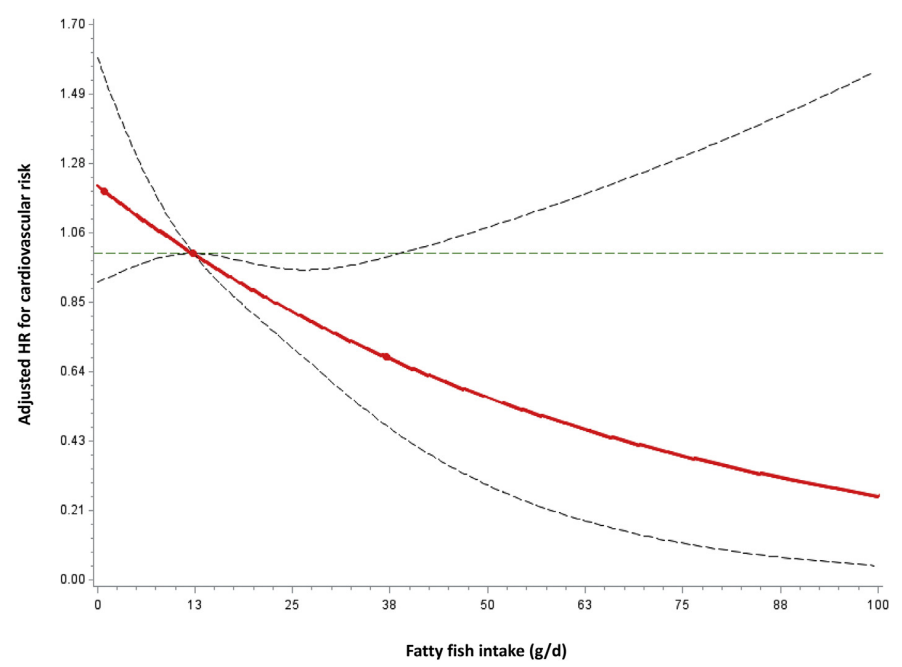

Figure 1 Risk of composite CHD and stroke associated with fatty fish intake (g/d) within the population of the Moli-sani study ( $\mathrm{n}=20,969$ ). Hazard ratios (95\% confidence interval) were adjusted for age, sex, energy intake, education, smoking, drugs for diabetes, drugs for hypertension, drugs for lipids, Mediterranean diet score without fish, blood glucose, LDL-cholesterol, low-grade inflammation, lean fish (g/d), dried fish (g/d) and other types of fish $(\mathrm{g} / \mathrm{d})$ not included in lean/fatty types.

corresponding to 8 times/week, was associated with reduced risk of coronary heart disease, primarily nonfatal cardiac events, as compared with those having fish 1 time per week, while no benefits were documented at lower intakes [9].

Cardiovascular benefits were observed also in the JACC study, in which a protection from cardiovascular death associated with greater fish intake was found [17], in agreement with previous findings on middle-aged Chinese men reporting lower risk of fatal acute myocardial infarction [18].

The positive association between fish intake and CVD outcomes has been documented also in low fishconsuming populations; the MORGEN study in the Netherlands showed that fish intake was likely to lower the risk of fatal CHD and myocardial infarction in a doseresponsive manner also in populations with a low habitual fish consumption, with protection from fatal CHD being evident even at modest intakes [10]. Similarly, in the Zutphen study at least one portion of fish per week was found to be associated with a reduced stroke incidence [19]. Data from US showed a reduced risk of ischaemic stroke in men at very low intakes (one per month or more) [20] and similar findings were observed in US women [21], although the Women's Health Study failed to find any association with risk of CVD [22].

Omega-3 fatty acids (eicosapentaenoic, EPA, and docosahexaenoic, DHA, acids) have been identified as the likely active constituents of fish and have numerous health benefits, such as pleiotropic protective cardiovascular effects, including lowering blood pressure, modulating serum lipids, improving diabetes and insulin resistance, reducing arrhythmia, suppressing platelet aggregation, improving endothelial function, and inhibiting inflammation $[23,24]$. However, advantages associated with omega3 fatty acids were mainly observed for high amounts, often not within the range of dietary intake of fish [7].

We also evaluated the association of a number of fish sub-types with incident CVD. No single fish type resulted associated with reduced CVD risk, although a trend toward an increased risk was found associated with greater consumption of dried fish: the latter is usually high in salt content, a major risk factor for CVD and in particular for stroke [25].

However, when distinction was made on the basis of fat content, higher intake of fatty fish only was found to be related to reduce incidence of the composite outcome and of CHD events. This finding is in line with prior observations on a Dutch population showing a lower sudden coronary death risk possibly associated with long-term fatty-fish consumption [26]. Within Italian men recruited in the Seven Countries study risk of CHD mortality too resulted lowered by consumption of fatty fish but not of lean fish [11].

The heart advantages associated with consumption of fatty fish are likely linked to beneficial effects on a number of CVD risk factors, such as triglycerides lowering and improvement of HDL-cholesterol levels, as shown by intervention trials $[27,28]$.

In our cohort, high intake of fish was not associated at baseline with better cardiovascular health profile. Indeed, individuals consuming greater amounts of fish had 
metabolic biomarker levels comparable to those in the lowest group of intake. Lipids, blood glucose levels and low-grade inflammation did not appear to be on the pathway of the association between fish intake and CVD risk, possibly because the high fish consumers had higher prevalence of the use of drugs for diabetes, hypertension and lipid-lowering medications. This may be possibly due at least in part to reverse causation with groups at high cardiovascular risk likely having shifted their diet, if not fish-dislike, by including more fish intake. The exclusion of subjects with prior CVD events is likely to limit such potential source of bias.

Further studies are needed to clarify the possible mechanisms underlying the relationship of fish intake with health outcomes by addressing a number of pathways possibly including both traditional and emerging CVD risk factors, such as low-grade inflammation.

\section{Strengths and limitations of the study}

Major strengths of this study include a large communitybased cohort, its prospective design, detailed information of dietary intake, and the considerable number of covariates, thus minimising sources of bias and confounding. It also shows, possibly for the first time, that high fish consumption is associated with a significant cardiovascular benefit in people already adhering to the Mediterranean Diet.

This study also presents several limitations. First, information on dietary intake was self-reported and can lead to under- or overestimates. Another limitation is represented by the relatively short follow-up duration and a consequent small number of incident CVD events. The observational nature of the study cannot allow to fully rule out residual confounding or confounding by unmeasured factors. Finally, subjects' information was collected at baseline only, thus life-course changes possibly occurred during the follow-up may have influenced the strength of our findings.

Data used in the present study have been collected in a region located between Central and Southern Italy, Mediterranean by tradition and culture but also representative of the general Italian population, thus caution is needed in extending the results to other contexts.

\section{Conclusions}

The favourable health benefits associated with fish intake are extensively documented in different epidemiological settings, especially in relation to CHD mortality risk reduction, and a dominant role of fatty fish over lean types clearly emerged in many studies. Our findings showed an inverse association of high fish intake with incidence of CVD, and confirmed a predominant role of fatty over lean fish. Few studies however had previously specifically addressed the relationship between fish intake and incidence of CVD in Mediterranean populations. To the best of our knowledge, this is one of the few studies examining longitudinally the association of total fish and fish types intake with risk of developing CVD in a general population, already exposed to the heart advantages associated with adherence to a Mediterranean dietary pattern.

\section{Sources of funding}

The enrolment phase of the Moli-sani study was supported by research grants from Pfizer Foundation (Rome, Italy), the Italian Ministry of University and Research (MIUR, Rome, Italy)-Programma Triennale di Ricerca, Decreto no.1588 and Instrumentation Laboratory, Milan, Italy. Funders had no role in study design, collection, analysis, and interpretation of data; in the writing of the manuscript and in the decision to submit the article for publication.

Marialaura Bonaccio was supported by a Fondazione Umberto Veronesi Fellowship.

Simona Costanzo is the recipient of a Fondazione Umberto Veronesi Travel Grant.

All Authors were and are independent from funders. The analyses included in this paper were partially supported by the Italian Ministry of Health 2013 (Young investigator grant to MB, number: GR-2013-02356060) and by the Italian Association for Cancer Research (A.I.R.C.) with grant AIRC “5x1000” Ref. n. 12237.

\section{Contribution}

$\mathrm{LI}, \mathrm{MB}$ and ER contributed to the conception and design of the work, and interpretation of data; SC, MP and ADeC managed data collection; $\mathrm{MB}, \mathrm{ADiC}$ analysed the data; $\mathrm{MB}$ and ER wrote the paper; MBD, GdG, CC and LI originally inspired the research and critically reviewed the manuscript. All authors approved the final version of the manuscript.

\section{Disclosure of conflicts of interest}

None of the Authors had a personal or financial conflict of interest.

\section{Acknowledgments}

The Moli-sani research group thanks the Associazione Cuore Sano Onlus (Campobasso, Italy) for its cultural and financial support.

\section{Appendix A. Supplementary data}

Supplementary data related to this article can be found at http://dx.doi.org/10.1016/j.numecd.2017.08.004.

\section{Appendix}

\section{Moli-sani Study Investigators}

The enrolment phase of the Moli-sani Study was conducted at the Research Laboratories of the Catholic University in Campobasso (Italy), the follow up of the Moli- 
sani cohort is being conducted at the IRCCS Neuromed, Pozzilli, Italy.

Steering Committee: Licia Iacoviello (Neuromed, Pozzilli, Italy), Chairperson, Maria Benedetta Donati and Giovanni de Gaetano (Neuromed, Pozzilli, Italy).

Safety and data monitoring Committee: Jos Vermylen (Catholic Univesity, Leuven, Belgio), Chairman, Ignacio De Paula Carrasco (Accademia Pontificia Pro Vita, Roma, Italy), Simona Giampaoli (Istituto Superiore di Sanità, Roma, Italy), Antonio Spagnuolo (Catholic University, Roma, Italy).

Event adjudicating Committee: Deodato Assanelli (Brescia, Italy), Vincenzo Centritto (Campobasso, Italy), Pasquale Spagnuolo and Dante Staniscia (Termoli, Italy).

Scientific and organizing secretariat: Francesco Zito (Coordinator), Americo Bonanni, Chiara Cerletti, Amalia De Curtis, Augusto Di Castelnuovo, Licia Iacoviello, Roberto Lorenzet*, Antonio Mascioli, Marco Olivieri and Domenico Rotilio.

Data management and analysis: Augusto Di Castelnuovo (Coordinator), Marialaura Bonaccio, Simona Costanzo and Francesco Gianfagna.

Informatics: Marco Olivieri (Coordinator), Maurizio Giacci, Antonella Padulo and Dario Petraroia.

Biobank and biomedical analyses: Amalia De Curtis (Coordinator), Sara Magnacca, Federico Marracino, Maria Spinelli, Christian Silvestri, Giuseppe dell'Elba, Claudio Grippi.

Communication and Press Office: Americo Bonanni (Coordinator), Marialaura Bonaccio and Francesca De Lucia.

Moli-family Project: Francesco Gianfagna, Branislav Vohnout.

Recruitment staff: Franco Zito (General Coordinator); Secretariat: Mariarosaria Persichillo (Coordinator), Angelita Verna, Maura Di Lillo, Irene Di Stefano; Blood sample: Agnieszka Pampuch; Branislav Vohnout, Agostino Pannichella, Antonio Rinaldo Vizzarri; Spirometry: Antonella Arcari (Coordinator), Daniela Barbato, Francesca Bracone, Simona Costanzo, Carmine Di Giorgio, Sara Magnacca, Simona Panebianco, Antonello Chiovitti, Federico Marracino, Sergio Caccamo, Vanesa Caruso; Electrocardiograms: Livia Rago (Coordinator), Daniela Cugino, Francesco Zito, Francesco Gianfagna, Alessandra Ferri, Concetta Castaldi, Marcella Mignogna, Tomasz Guszcz; Questionnaires: Romina di Giuseppe (Coordinator), Paola Barisciano, Lorena Buonaccorsi, Floriana Centritto, Antonella Cutrone, Francesca De Lucia, Francesca Fanelli, Iolanda Santimone, Anna Sciarretta, Maura Di Lillo, Isabella Sorella, Irene Di Stefano, Emanuela Plescia, Alessandra Molinaro and Christiana Cavone.

Call Center: Giovanna Galuppo, Maura Di Lillo, Concetta Castaldi, Dolores D'Angelo and Rosanna Ramacciato.

Follow-up: Simona Costanzo (Coordinator); Data management: Simona Costanzo, Marco Olivieri.

Event adjudication: Livia Rago (Coordinator), Simona Costanzo, Amalia De Curtis, Licia Iacoviello, Mariarosaria Persichillo.
Regional Health Institutions: Azienda Sanitaria Regionale del Molise (ASReM, Campobasso, Italy), UOC Servizio Igiene e Sanità Pubblica - Dipartimento di Prevenzione; Offices of vital statistics of the Molise region and Molise Dati Spa (Campobasso, Italy).

Hospitals: Presidi Ospedalieri ASReM (Presidio Ospedaliero A. Cardarelli - Campobasso; Ospedale F. Veneziale - Isernia; Ospedale San Timoteo - Termoli (CB); Ospedale Ss. Rosario - Venafro (IS); Ospedale Vietri - Larino (CB); Ospedale San Francesco Caracciolo - Agnone (IS)); Istituto di cura Villa Maria - Campobasso; Fondazione di Ricerca e Cura Giovanni Paolo II - Campobasso; IRCCS Neuromed Pozzilli (IS).

${ }^{*}$ Deceased

\section{References}

[1] Townsend N, Wilson L, Bhatnagar P, Wickramasinghe K, Rayner M, Nichols M. Cardiovascular disease in Europe: epidemiological update 2016. Eur Heart J 2016;37:3232-45.

[2] Bonaccio M, Di Castelnuovo A, Costanzo S, Persichillo M, De Curtis A, Donati MB, et al. Adherence to the traditional Mediterranean diet and mortality in subjects with diabetes. Prospective results from the MOLI-SANI study. Eur J Prev Cardiol 2016;23: 400-7.

[3] Bazzano LA, He J, Ogden LG, Loria CM, Vupputuri S, Myers L, et al. Fruit and vegetable intake and risk of cardiovascular disease in US adults: the first National Health and Nutrition Examination Survey Epidemiologic follow-up study. Am J Clin Nutr 2002;76:93-9.

[4] http://www.heart.org/HEARTORG/HealthyLiving/HealthyEating Nutrition/Meat-Poultry-and-Fish_UCM_306002_Article.jsp\#. WMkYpDvhA2w (Accessed March 2017).

[5] Zheng J, Huang T, Yu Y, Hu X, Yang B, Li D. Fish consumption and CHD mortality: an updated meta-analysis of seventeen cohort studies. Public Health Nutr 2012:15:725-37.

[6] Xun P, Qin B, Song Y, Nakamura Y, Kurth T, Yaemsiri S, et al. Fish consumption and risk of stroke and its subtypes: accumulative evidence from a meta-analysis of prospective cohort studies. Eur J Clin Nutr 2012:66:1199-207.

[7] Chowdhury R, Stevens S, Gorman D, Pan A, Warnakula S, Chowdhury S, et al. Association between fish consumption, long chain omega 3 fatty acids, and risk of cerebrovascular disease: systematic review and meta-analysis. BMJ 2012 Oct 30;345: e6698.

[8] Takata Y, Zhang X, Li H, Gao YT, Yang G, Gao J, et al. Fish intake and risks of total and cause-specific mortality in 2 population-based cohort studies of 134,296 men and women. Am J Epidemiol 2013;178:46-57.

[9] Iso H, Kobayashi M, Ishihara J, Sasaki S, Okada K, Kita Y, et al. Intake of fish and n 3 fatty acids and risk of coronary heart disease among Japanese: the Japan Public Health Center-Based (JPHC) Study Cohort I. Circulation 2006;113:195-202.

[10] De Goede J, Geleijnse JM, Boer JM, Kromhout D, Verschuren WM. Marine (n-3) fatty acids, fish consumption, and the 10 -year risk of fatal and nonfatal coronary heart disease in a large population of Dutch adults with low fish intake. J Nutr 2010;140:1023-8

[11] Oomen CM, Feskens EJ, Räsänen L, Fidanza F, Nissinen AM, Menotti A, et al. Fish consumption and coronary heart disease mortality in Finland, Italy, and The Netherlands. Am J Epidemiol 2000;151:999-1006.

[12] Amiano P, Chamosa S, Etxezarreta N, Arriola L, Moreno-Iribas C, Huerta JM, et al. No association between fish consumption and risk of stroke in the Spanish cohort of the European Prospective Investigation into Cancer and Nutrition (EPIC-Spain): a 13.8-year follow-up study. Public Health Nutr 2016;19:674-81.

[13] Di Castelnuovo A, Costanzo S, Persichillo M, Olivieri M, de Curtis A, Zito F, et al. Distribution of short and lifetime risks for 
cardiovascular disease in Italians. Eur J Prev Cardiol 2012;19: 723-30.

[14] Pisani P, Faggiano F, Krogh V, Palli D, Vineis P, Berrino F. Relative validity and reproducibility of a food frequency dietary questionnaire for use in the Italian EPIC centres. Int J Epidemiol 1997; 26(Suppl 1):S152-60.

[15] Trichopoulou A, Costacou T, Bamia C, Trichopoulos D. Adherence to a Mediterranean diet and survival in a Greek population. N Engl J Med 2003;348:2599-608.

[16] Luepker RV, Apple FS, Christenson RH, Crow RS, Fortmann SP Goff D, et al. Case definitions for acute coronary heart disease in epidemiology and clinical research studies: a Statement from the AHA Council on Epidemiology and Prevention; AHA Statistics Committee; World Heart Federation Council on Epidemiology and Prevention. Circulation 2003;108:2543-9.

[17] Yamagishi K, Iso H, Date C, Fukui M, Wakai K, Kikuchi S, et al. Fish, omega-3 polyunsaturated fatty acids, and mortality from cardiovascular diseases in a nationwide community-based cohort of Japanese men and women the JACC (Japan Collaborative Cohort Study for Evaluation of Cancer Risk) Study. J Am Coll Cardiol 2008; 52:988-96.

[18] Yuan JM, Ross RK, Gao YT, Yu MC. Fish and shellfish consumption in relation to death from myocardial infarction among men in Shanghai, China. Am J Epidemiol 2001;154:809-16.

[19] Keli SO, Feskens EJ, Kromhout D. Fish consumption and risk of stroke. The Zutphen Study. Stroke 1994;25:328-32.

[20] He K, Rimm EB, Merchant A, Rosner BA, Stampfer MJ, Willett WC et al. Fish consumption and risk of stroke in men. JAMA 2002;288: 3130-6.
[21] Gillum RF, Mussolino ME, Madans JH. The relationship between fish consumption and stroke incidence. The NHANES I Epidemiologic Follow-up Study (National Health and Nutrition Examination Survey). Arch Intern Med 1996;156:537-42.

[22] Rhee JJ, Kim E, Buring JE, Kurth T. Fish consumption, omega-3 fatty acids, and risk of cardiovascular disease. Am J Prev Med 2017;52: 10-9.

[23] Mozaffarian D, Rimm EB. Fish intake, contaminants, and human health: evaluating the risks and the benefits. JAMA 2006;296: 1885-99.

[24] Dietary supplementation with n-3 polyunsaturated fatty acids and vitamin $\mathrm{E}$ after myocardial infarction: results of the GISSIPrevenzione trial. Gruppo Italiano per lo Studio della Sopravvivenza nell'Infarto miocardico. Lancet 1999;354:447-55.

[25] Strazzullo P, D'Elia L, Kandala NB, Cappuccio FP. Salt intake, stroke and cardiovascular disease: meta-analysis of prospective studies. BMJ 2009 Nov 24;339:b4567.

[26] Streppl MT, Ocké MC, Boshuizen HC, Kok FJ, Kromhout D. Longterm fish consumption and n-3 fatty acid intake in relation to (sudden) coronary heart disease death: the Zutphen study. Eur Heart J 2008;29:2024-30.

[27] Appel LJ, Miller 3rd ER, Seidler AJ, Whelton PK. Does supplementation of diet with 'fish oil' reduce blood pressure? A metaanalysis of controlled clinical trials. Arch Intern Med 1993;153: $1429-38$.

[28] Moore CS, Bryant SP, Mishra GD, Krebs JD, Browning LM, Miller GJ et al. Oily fish reduces plasma triacylglycerols: a primary prevention study in overweight men and women. Nutrition 2006;22:1012-24. 



\section{CHAPTER 6.}

Egg consumption and risk of total and cause-specific mortality in a healthy Mediterranean population

Ruggiero E, Di Castelnuovo A, Costanzo S, Persichillo M, De Curtis A, Cerletti C, Donati MB, de Gaetano G, Iacoviello L, Bonaccio M, on behalf of the Moli-sani study Investigators.

Submitted 



\section{European Journal of Nutrition \\ Egg consumption and risk of total and cause-specific mortality in a healthy Mediterranean population \\ --Manuscript Draft--}

\begin{tabular}{|c|c|}
\hline Manuscript Number: & EJON-D-20-01323 \\
\hline Full Title: & $\begin{array}{l}\text { Egg consumption and risk of total and cause-specific mortality in a healthy } \\
\text { Mediterranean population }\end{array}$ \\
\hline Article Type: & Original Contribution \\
\hline Keywords: & eggs; dietary cholesterol; mortality risk; Mediterranean Diet \\
\hline Corresponding Author: & $\begin{array}{l}\text { Licia lacoviello } \\
\text { NEUROMED } \\
\text { ITALY }\end{array}$ \\
\hline \multicolumn{2}{|l|}{$\begin{array}{l}\text { Corresponding Author Secondary } \\
\text { Information: }\end{array}$} \\
\hline Corresponding Author's Institution: & NEUROMED \\
\hline \multicolumn{2}{|l|}{$\begin{array}{l}\text { Corresponding Author's Secondary } \\
\text { Institution: }\end{array}$} \\
\hline First Author: & Emilia Ruggiero \\
\hline \multicolumn{2}{|l|}{ First Author Secondary Information: } \\
\hline \multirow[t]{10}{*}{ Order of Authors: } & Emilia Ruggiero \\
\hline & Augusto Di Castelnuovo \\
\hline & Simona Costanzo \\
\hline & Mariarosaria Persichillo \\
\hline & Amalia De Curtis \\
\hline & Chiara Cerletti \\
\hline & Maria Benedetta Donati \\
\hline & Giovanni de Gaetano \\
\hline & Licia lacoviello \\
\hline & Marialaura Bonaccio \\
\hline \multicolumn{2}{|c|}{ Order of Authors Secondary Information: } \\
\hline \multicolumn{2}{|l|}{ Funding Information: } \\
\hline Abstract: & $\begin{array}{l}\text { Purpose: Dietary guidelines recommend to limit egg consumption to } 4 \text { servings per } \\
\text { week but the relation between egg intake and health outcomes is still controversial. To } \\
\text { evaluate the association of egg consumption and mortality risk in Italian adults and to } \\
\text { investigate nutritional factors and serum lipids as potentially explaining such } \\
\text { associations. } \\
\text { Methods: Longitudinal analysis on } 20,564 \text { men and women aged } \geq 35 y \text {, free from } \\
\text { cardiovascular disease (CVD) and cancer belonging to the Moli-sani Study cohort } \\
\text { (enrolled } 2005-2010 \text { ) followed up for a median of } 8.2 \text { years. } \\
\text { Results: In multivariable-adjusted analysis as compared to low intake }(0-1 \text { egg/week), } \\
\text { eating }>4 \text { eggs/week led to an increased risk of all-cause (Hazard ratio [HR] }=1.49 ; \\
95 \% \mathrm{Cl} 1.13-1.99) \text {, CVD (HR=1.72; } 1.05-2.82) \text { and cancer mortality (HR=1.52; } 0.99- \\
2.34) \text {. Similarly, an intake of } 2-4 \text { eggs/week was associated with higher all-cause } \\
\text { (HR=1.22; } 1.01-1.46 \text { ) and CVD mortality risk (HR=1.44; } 1.04-1.99) \text {. An increase of } 1 \\
\text { egg per week was associated with higher mortality risk among high-risk individuals, } \\
\text { such as those with hypertension and hyperlipidaemia. } \\
\text { Dietary cholesterol explained about } 30 \% \text { of the association of eggs with all-cause and } \\
\text { CVD mortality while serum lipids ( e.g., total cholesterol) accounted for a small }\end{array}$ \\
\hline
\end{tabular}


proportion of egg-mortality relation.

Conclusions: Eating more than four eggs per week was associated with a markedly

increased risk of mortality in a Mediterranean population. However, a significant

increased mortality risk is also associated with consuming 2-4 eggs/week, an amount

recommended instead by several dietary guidelines. A substantial part of this

association was likely due to the egg contribution to dietary cholesterol. 
Egg consumption and risk of total and cause-specific mortality in a healthy Mediterranean population

Emilia Ruggiero $^{1}$, Augusto Di Castelnuovo ${ }^{2}$, Simona Costanzo $^{1}$, Mariarosaria Persichillo ${ }^{1}$, Amalia De Curtis $^{1}$, Chiara Cerletti ${ }^{1}$, Maria Benedetta Donati ${ }^{1}$, Giovanni de Gaetano ${ }^{1}$, Licia Iacoviello ${ }^{1,3}$ and Marialaura Bonaccio ${ }^{1}$, on behalf of the Moli-sani Study Investigators.

${ }^{1}$ Department of Epidemiology and Prevention, IRCCS NEUROMED, Pozzilli (IS), Italy.

${ }^{2}$ Mediterranea Cardiocentro, Napoli, Italy.

${ }^{3}$ Research Centre in Epidemiology and Preventive Medicine (EPIMED), Department of Medicine and Surgery, University of Insubria, Varese-Como, Italy.

*Moli-sani Study Investigators are listed in the Supplementary material

\section{Corresponding Author:}

Licia Iacoviello, $\mathrm{MD}, \mathrm{PhD}$

Department of Epidemiology and Prevention

IRCCS NEUROMED

Via dell'Elettronica, 86077 Pozzilli (IS), Italy.

Mail to: licia.iacoviello@moli-sani.org

$\underline{\text { licia.iacoviello@neuromed.it }}$ 


\begin{abstract}
Purpose: Dietary guidelines recommend to limit egg consumption to 4 servings per week but the relation between egg intake and health outcomes is still controversial. To evaluate the association of egg consumption and mortality risk in Italian adults and to investigate nutritional factors and serum lipids as potentially explaining such associations.
\end{abstract}

Methods: Longitudinal analysis on 20,564 men and women aged $\geq 35 \mathrm{y}$, free from cardiovascular disease (CVD) and cancer belonging to the Moli-sani Study cohort (enrolled 2005-2010) followed up for a median of 8.2 years.

Results: In multivariable-adjusted analysis as compared to low intake (0-1 egg/week), eating >4 eggs/week led to an increased risk of all-cause (Hazard ratio [HR]=1.49; 95\%CI 1.13-1.99), CVD $(\mathrm{HR}=1.72 ; 1.05-2.82)$ and cancer mortality $(\mathrm{HR}=1.52 ; 0.99-2.34)$. Similarly, an intake of 2-4 eggs/week was associated with higher all-cause (HR=1.22; 1.01-1.46) and CVD mortality risk $(\mathrm{HR}=1.44 ; 1.04-1.99)$. An increase of 1 egg per week was associated with higher mortality risk among high-risk individuals, such as those with hypertension and hyperlipidaemia.

Dietary cholesterol explained about $30 \%$ of the association of eggs with all-cause and CVD mortality while serum lipids (e.g., total cholesterol) accounted for a small proportion of egg-mortality relation. Conclusions: Eating more than four eggs per week was associated with a markedly increased risk of mortality in a Mediterranean population. However, a significant increased mortality risk is also associated with consuming 2-4 eggs/week, an amount recommended instead by several dietary guidelines. A substantial part of this association was likely due to the egg contribution to dietary cholesterol.

Keywords: eggs; dietary cholesterol; mortality risk; Mediterranean diet 


\section{INTRODUCTION}

Eggs are a common food in every diet and are used in many basic and formulated preparations. Eggs are affordable and nutrient-dense food items, containing high-quality protein with low levels of saturated fatty acids, and are rich in several micronutrients including vitamins and minerals, some of which (vitamin E, carotenoids) are reported to have antioxidant properties [1].

On the other side, eggs are also major sources of dietary cholesterol, a potential risk factor for cardiovascular health [2]. Dietary guidelines for egg consumption have changed several times over the past few decades, and vary among health agencies [3,4]. In 2010, the Dietary Guidelines for Americans recommended cholesterol intake to be limited to no more than 300 milligrams per day [5], but the 2015-2020 Dietary Guidelines Advisory Committee did not bring forward this recommendation, due to the lack of evidence of an appreciable relationship between consumption of dietary cholesterol and serum cholesterol, a decision consistent with the conclusions of the AHA/ACC report [6].

Based on their high cholesterol content, the Mediterranean Diet Foundation recommends to consume up to 4 eggs per week, as a healthy alternative to fish or meat [7], and the same amount (2-4 eggs per week) was indicated in the latest Italian dietary guidelines [8]. In the Eatwell Guide issued by the National Health Service in UK there is no recommended limit on how many eggs people should eat [9].

Epidemiological studies analysing the association of eggs with health outcomes have provided controversial results [10-12]. Several meta-analyses and an umbrella review of observational studies failed to report significant associations between egg intake and cardiovascular outcomes, even highlighting a downward trend association with stroke risk [13-16].

Data on the association of egg intake with mortality risk is limited and controversial [17-19], and the scientific evidence for the recommendations on dietary cholesterol and eggs is inconsistent and 
lacking in Mediterranean countries where the association between eggs and health has been seldom explored $[10,20,21]$.

In light of this, our study aimed first to longitudinally evaluate the association of egg consumption with total and cause-specific mortality in a large Mediterranean population of adult men and women; second, to investigate whether dietary cholesterol and other nutritional factors could be on the pathway between egg intake and mortality risk.

\section{MATERIALS AND METHODS}

\section{Study population}

Data are from the Moli-sani Study cohort established in 2005-2010 in the Molise region, a Mediterranean area in Italy, that recruited 24,325 men and women aged $\geq 35$ years [22]. For the purpose of the present analyses, we excluded subjects with history of CVD $(n=1,537)$ or missing data on CVD $(n=360)$, cancer $(n=777)$ or missing data on cancer $(n=89)$, those individuals with missing data for egg intake $(n=100)$, those reporting implausible energy intakes $(<800 \mathrm{kcal} / \mathrm{d}$ in men and $<500 \mathrm{kcal} / \mathrm{d}$ in women or $>4000 \mathrm{kcal} / \mathrm{d}$ in men and $>3500 \mathrm{kcal} / \mathrm{d}$ in women; $\mathrm{n}=771$ ), dietary or medical questionnaires judged as unreliable by interviewers ( $n=955$ and $n=235$, respectively), subjects lost to follow-up ( $\mathrm{n}=23)$ and missing information on cause-specific death $(\mathrm{n}=45)$. We finally analysed 20,564 individuals $(82.6 \%$ of the study sample). The Moli-sani Study cohort was followed up until December $31^{\text {st }} 2015$ with the main outcome of interest being mortality. Overall and cause-specific mortality was assessed by the Italian mortality registry (ReNCaM registry), validated by Italian death certificates (ISTAT form) and coded according to the International Classification of Diseases (ICD-9 version). Cardiovascular (CVD) mortality included deaths from diseases of the circulatory system, when the underlying cause of death included ICD9 codes 390-459. ICD-9 codes 430-438 were used to define specific cause of death for cerebrovascular disease, ICD-9 codes 410-414 and 429 for ischemic heart 
disease (IHD). Cancer death was considered when the underlying cause of death included ICD9 codes 140-208. Non cardiovascular/non cancer causes of death were included in 'other cause mortality' group.

The Moli-sani Study complies with the Declaration of Helsinki and was approved by the ethical committee of the Catholic University Medical School in Rome, Italy. All participants provided written informed consent.

\section{Dietary assessment}

Dietary intake was assessed by a trained interviewer-administered semi-quantitative EPIC food frequency questionnaire (FFQ) validated and adapted to the Italian population to assess participants' diet during the past 12 months.

The FFQ includes 188 food items, classified into 74 predefined food groups on the basis of similar nutrient characteristics or culinary usage [23]. Participants were asked to indicate the number of times a given item was consumed (per day, week, month or year) from which the frequency of consumption was calculated. The quantity of food consumed was assessed by asking the participant to select one among several images of different food portions or a predefined standard portion, when no image was available.

Frequencies and quantities of each food were then linked to Italian Food Tables [24], using a specifically designed software [25], to obtain quantitative estimates of daily intake of macro- and micro-nutrients plus energy.

Total egg consumption from various food sources (e.g. whole egg, omelette) was defined as number per week (we used $50 \mathrm{~g}$ as the standard weight for one medium-sized egg) and categorized as up to 1 egg/week, $>1 \leq 2$ eggs/week, $>2 \leq 4$ eggs/week and $>4$ eggs/week or used as a continuous variable as a legg increment per week. 
Main macronutrients contained in eggs (saturated fatty acids and protein, expressed as $\mathrm{g} / \mathrm{d}$ ) and dietary cholesterol $(\mathrm{mg} / \mathrm{d})$, calculated as mentioned above, were tested as potential mediators of the association between egg intake and mortality. Moreover, we similarly calculated and tested dietary vitamin $\mathrm{E}(\mathrm{mg} / \mathrm{d})$ and beta-carotenoids $(\mu \mathrm{g} / \mathrm{d})$, two micronutrients present in egg yolk that have been reported to have antioxidant properties [1].

Adherence to the traditional Mediterranean diet (MD) was evaluated by the Mediterranean Diet Score (MDS) developed by Trichopoulou et al. [26].

\section{Assessment of covariates}

At baseline, information on socio-demographic variables, lifestyles and medical history were obtained by interviewer-administered questionnaires.

Participants were considered to have diabetes, hypertension or hyperlipidaemia at baseline if they were taking disease-specific drugs.

Leisure-time physical activity (PA) was calculated for sport activity, walking and gardening, and then dichotomized as $<$ or $\geq 30 \mathrm{~min} / \mathrm{d}$. Height and weight were measured and body mass index (BMI) was calculated as $\mathrm{kg} / \mathrm{m}^{2}$ and then grouped into three categories as normal $(\leq 25)$, overweight $(25-30)$ or obese $(\geq 30)$.

Subjects were classified as never-smokers, current smokers or former smokers (quit at least 1 year ago). Education was based on the highest qualification attained and was categorized as up to lower secondary (approximately $\leq 8$ years of study), upper secondary school (8-13 years of study) and postsecondary education ( $>13$ years of study).

Urban or rural environments were defined on the basis of the urbanization level as described by the European Institute of Statistics (EUROSTAT definition) and obtained by the tool 'Atlante Statistico dei Comuni’ provided by the Italian National Institute of Statistics [27]. 
Venous blood samples were obtained from participants who had fasted overnight and had refrained from smoking for at least $6 \mathrm{~h}$.

Serum lipids (total cholesterol, HDL-cholesterol, triglycerides) were assayed by enzymatic reaction methods using an automatic analyzer (ILab 350, Instrumentation Laboratory (IL), Milan, Italy). Low-density lipoprotein (LDL) cholesterol was calculated according to Friedewald.

Quality control (high and low levels) for lipids was obtained by a commercial standard provided by the IL and an in-house serum standard pool. The coefficients of variability were respectively for high, medium and low values $4.9 \%, 5.2 \%$ and $4 \%$ for blood cholesterol; $3.2 \%, 3 \%$ and $4.5 \%$ for HDL-cholesterol; 5.2\%, 5.3\% and 5\% for triglycerides.

\section{Statistical analysis}

Baseline characteristics of the study population by categories of egg intake were presented as means with standard deviation (SD), or number and percentages (Table 1).

Associations for continuous and categorical variables were estimated by using general linear models adjusted for age, sex and energy intake (kcal/d) (PROC GENMOD and PROC GLM in SAS for categorical and continuous variables, respectively; Table 1 and Supplementary Table 1).

Risk estimates for all-cause and cause-specific deaths were expressed as hazard ratios (HR) with 95\% confidence intervals (95\% CI) and calculated by using Cox regression models with time-onstudy on the time scale and adjusting for baseline age as covariate in the model.

Based on previous literature and biological plausibility, two multivariable models were fitted to assess the association between egg intake and mortality: Model 1 was adjusted for age (continuous), sex and energy intake (kcal; continuous); Model 2 as in model 1 further controlled for education (up to lower secondary school; upper secondary school; postsecondary/higher), residence (urban, rural), leisure-time PA ( $<30$ or $\geq 30 \mathrm{~min} / \mathrm{d}$ ), smoking (never, current, former), BMI (normal, overweight, obese), diabetes (no, yes, missing), hyperlipidaemia (no, yes, missing), hypertension (no, yes, 
missing); Model 3 as in model 2 further controlled for MDS (continuous). Missing values for diabetes $(n=242)$, hypertension $(n=131)$ and hyperlipidaemia $(n=148)$ were included in the models as dummy variables, similar to the way valid categories were represented. For education, BMI and smoking (less than $1 \%$ of missing values) missing values were imputed to the modal value. Mediation analysis was used to estimate the possible contribution of nutritional factors and serum lipids (total blood cholesterol, HDL-cholesterol, LDL-cholesterol, triglycerides) in accounting for the association of egg intake with mortality. For the mediation analysis we used the \%MEDIATE macro in SAS software [28] to calculate the point and interval estimates of the percent of exposure effect (PTE) explained by one or more intermediate variables, with $95 \%$ confidence interval and $\mathrm{P}$ values. Nutritional factors and serum lipids were entered into the mediation analysis as continuous variables and positively skewed variables (triglycerides) were log transformed before analysis. Mediation analyses with biomarkers were restricted to 20,148 subjects after exclusion of those individuals with missing data on any of the biomarker.

To increase the applicability of the study results we calculated risk estimations for each additional $186 \mathrm{mg}$ of dietary cholesterol per day, $6.2 \mathrm{~g}$ of dietary proteins per day, $1.6 \mathrm{~g}$ of saturated fatty acids per day, $0.56 \mathrm{mg}$ of vitamin E per day and $42 \mu \mathrm{g}$ per day of beta-carotene, since these are the amounts of nutrients present in 1 egg, based on data from Food Composition Database for Epidemiological studies [29].

We performed sub-group analysis according to levels of intake of each food group included into the MDS (above/below the study population median; supplementary Table 3). Furthermore, we conducted subgroup analyses to test the robustness of the observed associations to various baseline risk factors: age (35-64 and $\geq 65$ years), sex, education, lifestyles (smoking status, leisure-time PA), health conditions at baseline (BMI, diabetes, hypertension and hyperlipidaemia), and after excluding those subjects who died during the first 2 years of follow-up (Supplementary Table 4). Appropriate 
multiplicative terms for testing interaction were included in the multivariable-adjusted models to test for a difference of effect of egg intake across subgroups.

The data analysis was generated using SAS/STAT software, Version 9.4 of the SAS System for Windows@2009.

\section{RESULTS}

In this Moli-sani population the mean consumption was 1.8 eggs per week $(\mathrm{SD} \pm 1.3)$, while overall mean dietary cholesterol consumption was $322 \mathrm{mg} / \mathrm{d}(\mathrm{SD} \pm 108)$. Egg contributed to $14.7 \%$ of total cholesterol intake in the diet.

Compared to low intake (0-1 eggs/week), consumers of $>4$ eggs/week had less CVD risk factors (i.e. BMI, diabetes, hypertension and hyperlipidaemia), including lower levels of blood lipids (Table 1), tended to be less close to an MD and globally consumed less fruits and nuts, cereals, and more meat and meat products (Supplementary Table 1).

The contribution of proteins and fats to total energy intake increased across categories of egg consumption, whereas the contribution of carbohydrates and fibre decreased; dietary cholesterol increased according to egg intake, as well as vitamin E levels while no substantial differences were observed for beta-carotene levels (Supplementary Table 1).

The cohort of 20,564 participants was followed-up for a median of 8.3 years (interquartile range $=7.4-9.3 \mathrm{y} ; 170,049$ person-years) during which 838 deaths were ascertained and validated; 271 from CVD, of which 153 were from IHD/cerebrovascular disease, 334 from cancer, and 233 from other causes. In a multivariable-adjusted model including also MDS, as compared to participants reporting lower egg consumption, consuming more than 4 eggs/week was associated with increased risk of all-cause $(\mathrm{HR}=1.49 ; 95 \% \mathrm{CI} 1.13-1.99), \mathrm{CVD}(\mathrm{HR}=1.72 ; 1.05-2.82)$ and cancer mortality $(\mathrm{HR}=1.52 ; 0.99-2.34)$; an upward trend of risk with $\mathrm{IHD} /$ cerebrovascular disease associated with increased egg intake was also observed $(\mathrm{HR}=1.57 ; 0.78-3.13)$, although statistical significance was 
not hold (Table 2, models 3). The recommended intake of 2-4 eggs/week was also associated with higher all-cause $(\mathrm{HR}=1.22 ; 1.01-1.46)$ and $\mathrm{CVD}$ mortality risk $(\mathrm{HR}=1.44 ; 1.04-1.99)($ Table 2 , models 3).

Multivariable-adjusted Kaplan-Meier estimates for all-cause mortality and CVD mortality for the four categories of egg consumption are well- separated (Figures 1A and 1B) and showed increased mortality risk associated with higher egg intake $(\mathrm{p}=0.030$ and $\mathrm{p}=0.083$ respectively).

Each additional egg per week was associated with higher risk of all-cause $(\mathrm{HR}=1.06 ; 1.00-1.12)$ and CVD mortality $(\mathrm{HR}=1.10 ; 1.01-1.20)$ and slightly with mortality risk from IHD/cerebrovascular causes $(\mathrm{HR}=1.11 ; 0.99-1.26$; Table 2, models 3$)$.

Dietary cholesterol inversely correlated with total serum cholesterol levels (Spearman correlation coefficients $r=-0.019, \mathrm{p}=0.0051)$, HDL-cholesterol $(\mathrm{r}=-0.025, \mathrm{p}=0.0004)$ and triglycerides $(\mathrm{r}=-0.045$, $\mathrm{p}<0.0001)$ and positively with saturated fat $(\mathrm{r}=0.83 ; \mathrm{p}<0.0001)$, but was not related to LDLcholesterol ( $r=-0.00065, \mathrm{p}=0.93$; data not shown).

Each additional $186 \mathrm{mg}$ of dietary cholesterol consumed per day was associated with higher risk of all-cause $(\mathrm{HR}=1.28 ; 95 \% \mathrm{CI} 1.08-1.52)$ and CVD mortality $(\mathrm{HR}=1.39 ; 95 \%$ CI 1.03-1.88;

Supplementary Table 2, model 2) although the strength of the association was reduced after inclusion of other nutrients into the model. Similarly, saturated fats intake was associated with increased risk of total and CVD mortality, although adjustment for other dietary factors mitigated the magnitude of the association (Supplementary Table 2).

Sub-group analyses indicated that overall diet quality, measured by the MDS, and single food groups were not effect modifiers of the relation between eggs and mortality risk (all $\mathrm{p}$ values for interaction $>0.05$; Supplementary Table 3), although an increased risk of CVD mortality associated with 1-egg increment per week was found among low fruit consumers as compared to high consumers ( $\mathrm{p}$ for interaction $=0.0038$, supplementary Table 3 ). 
Among baseline risk factors, hyperlipidaemia and hypertension were likely to modify the magnitude of the association between egg consumption (1-egg increment per week) and CVD mortality risk, resulting to be stronger in those using lipid-lowering drugs ( $\mathrm{p}$ for interaction=0.012) and among participants taking antihypertensive medications ( $\mathrm{p}$ for interaction $=0.045$; Supplementary Table 4).

\section{Mediation analysis}

We tested three nutritional factors prevalently present in eggs and two sources of antioxidants as possibly mediating the association with mortality. Dietary cholesterol explained $32.5 \%(\mathrm{p}<0.0001)$ and $29.0 \%(\mathrm{p}<0.0001)$ of the association of egg intake with all-cause and CVD mortality risk, respectively; saturated fats accounted from $9.9 \%$ to $8.0 \%$, while dietary protein offered little contribution (Table 3). Antioxidant content was not on the egg-mortality pathway. Baseline differences in serum lipids across categories of egg intake only marginally accounted for the association of eggs with CVD mortality, while explained $6.5 \%(p<0.0001)$ and $5.4 \%(p=0.0003)$ of the relation between high egg consumption and all-cause and CVD mortality risk (Table 3).

\section{DISCUSSION}

In a large cohort of healthy adult Mediterranean individuals, eating more than 4 eggs per week was associated with an increased risk of all-cause and CVD mortality, in comparison with a lower intake (up to 1 egg per week) and independently of overall diet quality as reflected by adherence to the Mediterranean diet. Of interest, we found that even moderate egg intake of 2-4 servings per week, which is generally recommended by international dietary guidelines, led to an increased risk of allcause and CVD mortality by $22 \%$ and $44 \%$, respectively.

Egg and dietary cholesterol intakes in our cohort were similar to that reported in European countries from EPIC study [30] and in some US cohorts [31,32], but egg consumption in our cohort was 
slightly lower than that documented in other Mediterranean cohorts, which reported an average intake of 3-4 eggs per week $[9,19]$.

Our findings expand the scarce literature on this topic in Mediterranean settings. Consumption of 10 grams of egg per day (corresponding to approximately 1 and a half egg per week) was found associated with increased risk of total (31\%) and CVD death (54\%) in diabetic patients from the Greek arm of the EPIC study [21], while in the Spanish cohort of EPIC no association between egg consumption (up to 7 egg/week) and all-cause, CVD and IHD of mortality was found; yet, inverse associations between egg intake and risk of death from other causes ( $24 \%$ reduction) and from the nervous system diseases (41\%) were documented [20]. In the Spanish SUN cohort, no association was found between egg consumption and the incidence of CVD [9].

Studies from non-Mediterranean cohorts also yielded inconsistent findings. In a recent analysis from the NHANES cohort no significant associations between egg consumption and mortality in US adults were observed [33]; on the contrary, pooled data from 6 US based cohorts showed that each additional half an egg consumed per day was significantly associated with $6 \%$ higher risk of incident CVD and $8 \%$ of all-cause mortality [30].

Moreover, recent findings from nine European countries showed that higher egg consumption was associated with higher risk of haemorrhagic stroke (25\%) and an upward trend of increased ischaemic stroke risk [30], and in a Japanese cohort of women, a direct association was reported between egg intake ( $\geq 2$ eggs/d vs 1 egg/d) and total mortality [17]. A meta-analysis of 14 studies involving 320,778 subjects identified a dose-response positive association between egg consumption and the risk of CVD and diabetes [12], but more recently an analysis from three large US cohorts and an updated meta-analysis including 28 prospective studies showed that moderate egg consumption (up to 7 eggs per week) was not associated with cardiovascular disease risk overall [34]. 
Recently, a dose-response meta-analysis of prospective cohort studies found no association with risk of cardiovascular outcomes following the habitual consumption of one egg per day compared to no intake, with exception of the risk of heart failure, which resulted higher especially in men from US cohorts [35].

Inconsistencies across studies may be due to differences in population characteristics, sample sizes, cooking methods for eggs, or differences in dietary patterns related to different amounts of egg consumption and also different adjustments for confounders.

Our study relied on a comprehensive assessment of nutritional factors (e.g. saturated fats and overall diet quality) and also on a number of other potential confounding variables, such as lifestyles and socio-demographic factors.

We also observed an increased risk of cancer mortality associated with eating more than 4 eggs per week and this is in line with a dose-response meta-analysis of prospective observational studies showing that eating $\geq 5$ eggs per week may be associated with a modestly elevated risk of breast, ovarian and fatal prostate cancers [36]. Similarly, Japanese women consuming more than 2 eggs per day, as compared to those having $1 \mathrm{egg} / \mathrm{d}$, tripled their risk of dying from cancer [17]. Generally, eggs are a controversial food because of their saturated fat (about $3 \mathrm{~g} / 100 \mathrm{~g}$ ) and cholesterol content (about $370 \mathrm{mg} / 100 \mathrm{~g}$ ) [29] and on this basis experts have produced mounting evidence against frequent egg consumption due their potential association with CVD [31]. Indeed, pooled data from 6 US based cohorts recently showed that each additional $300 \mathrm{mg}$ of dietary cholesterol consumed per day was significantly associated with $17 \%$ and $18 \%$ higher risk of incident CVD and all-cause mortality, respectively [31], while others found that the dietary cholesterolmortality relation likely depends on the baseline intake levels, with an inverse association in those with lower intake levels $(<250 \mathrm{mg}$ /day) but a positive association in those with higher intake levels ( $\geq 250 \mathrm{mg} /$ day) [33]. 
In our study, we found that a substantial part of the excess risk of all-cause and CVD mortality associated with egg intake was accounted for by dietary cholesterol that, in turn, was associated with an increased risk of all-cause and CVD mortality, although the strength of the relation was reduced in multivariable-adjusted models. Our data are in accordance with observational data from US cohorts reporting that dietary cholesterol largely explained the association of eggs with increased CVD mortality [31].

However, the potential health risk of high dietary cholesterol levels has been questioned [37, 38] and more recently a meta-analysis concluded that available evidence is too heterogeneous and actually lacks methodologic rigor to draw any definitive conclusion regarding the influence of dietary cholesterol on CVD risk [37].

Since cholesterol-containing foods are usually rich in saturated fat and animal protein, which have been associated with increased CVD mortality risk in previous reports $[39,40]$, we also accounted for such nutrients but found that proteins were unlikely to be on the pathway between eggs and mortality risk, while saturated fats played a limited role. These results should be interpreted in light of the fact that eggs contain high-quality protein with minimal saturated fatty acids. Differences at baseline in serum lipids among study participants were unlikely to explain the excess of CVD risk associated with frequent egg intake, while explaining about $7 \%$ of the relation with total mortality. Individuals eating eggs more frequently tended to have lower levels of serum lipids; this may be counter-intuitive, but epidemiological evidence on a direct association between serum lipids and disease/death risk is inconsistent and not fully elucidated [41,42].

\section{Strengths and limitations}

To our knowledge, this is one of the largest prospective cohort studies evaluating the association between egg consumption and mortality in a Mediterranean population, and one of the few examining the nutrient-related mediating pathways between egg intake and mortality. 
Major strengths of this study include a large community-based cohort, its prospective design, detailed information of dietary intake and the considerable number of covariates allowing to minimize sources of bias and confounding.

However, this study also suffers of several limitations: first, the observational nature of the study cannot allow to fully rule out residual confounding or confounding by unmeasured factors. Second, information on dietary intake was self-reported and can lead to under- or over-estimates; moreover, subjects' dietary information was collected at baseline only, thus life-course changes possibly occurred during the follow-up period, may have influenced the strength of our findings. Also, we do not have data on preferred cooking methods which may likely influence the egg-mortality association.

Finally, participants lived in Molise, a region located between central and southern Italy, that its traditionally Mediterranean in culture; thus caution is needed in extending our results to other geographical and cultural contexts, although the main characteristics of our population sample are comparable to those of the Italian Cardiovascular Epidemiological Observatory and therefore representative of at least the Italian population [43].

\section{Conclusions}

Our findings report an increased risk of all-cause, CVD and cancer mortality associated with intake of more than 4 eggs per week in a large cohort of Italians from the general population. Increased death risk was also observed at lower intakes, namely 2-4 servings per week, which correspond to the egg intake recommended by many health advisory bodies and international dietary guidelines. The adverse health effects of eggs were found to be independent of the overall quality of the diet, while part of the excess of all-cause and CVD mortality risk was likely due to the high content of cholesterol and saturated fats of eggs, which in turn were found predictive of higher mortality risk in 
our cohort. As dietary cholesterol was not directly related to serum lipids, its adverse health effect may likely pass through biological mechanisms other than cholesterol-related ones.

Although we do acknowledge the limitations of our observational study, our findings are unlikely to be supportive of the current dietary guidelines most of which recommending a safe use up to 4 eggs per week, or even no type of restriction.

Finally, caution should be taken especially for high-risk individuals, such as those with hypercholesterolemia and hypertension. 


\section{ACKNOWLEDGMENTS}

The Moli-sani Study research group thanks the Associazione Cuore Sano Onlus (Campobasso, Italy) for its financial and cultural support, and is grateful to the BIOMARCARE Consortium.

Authors' contribution: ER, MB, and LI contributed to the design of the study and interpretation of data; SC, ADeC and MP managed data collection; ER, MB and $\mathrm{ADiC}$ analysed the data; $\mathrm{ER}$ and $\mathrm{MB}$ wrote the manuscript; MBD, CC, GdG and LI originally inspired the research and critically reviewed the manuscript. All Authors have read and approved the manuscript.

\section{Funding Source:}

The enrolment phase of the Moli-sani Study was supported by unrestricted research grants from the Pfizer Foundation (Rome, Italy), the Italian Ministry of University and Research (MIUR, Rome, Italy)-Programma Triennale di Ricerca, Decreto no.1588 and Instrumentation Laboratory, Milan, Italy. The follow-up phase of the Moli-sani Study (assessment of incident cases) was partially supported by AIRC “5xMILLE” (HYPERCAN Study, n. 12237) and the Italian Ministry of Health (PI GdG, CoPI SC; grant n. RF-2018-12367074). MB was supported by a Fondazione Umberto Veronesi Fellowship. SC was the recipient of a Fondazione Umberto Veronesi travel grant. The present analyses were partially supported by a grant to MB of the Italian Ministry of Health 2013 [Grant number GR-2013-02356060], by the Hypercan Study, AIRC “5xMILLE” n.12237 and by POR FESR 2014-2020: DD n. 459 del 27/11/2018. SATIN: Sviluppo di Approcci Terapeutici INnovativi per patologie neoplastiche resistenti ai trattamenti and by a grant to LI as a partner of BiomarCaRE (Biomarkers for Cardiovascular Risk Assessment in Europe) by European Commission Seventh Framework Programme FP7/2007-2013 [HEALTH-F2-2011-278913]. Funders had no role in study design, collection, analysis, and interpretation of data; in the writing of the manuscript and in the decision to submit the article for publication. All Authors were and are independent from funders.

Conflict of interest statement: None of the Authors has conflicts of interest to disclose. 


\section{REFERENCES}

1. Nimalaratne, C \& Wu J (2015) Hen Egg as an Antioxidant Food Commodity: A Review. Nutrients, 7(10), 8274-8293. https://doi.org/10.3390/nu7105394

2. Shekelle RB, Stamler J (1989). Dietary cholesterol and ischaemic heart disease. Lancet (London, England), 1(8648), 1177-1179. https://doi.org/10.1016/s0140-6736(89)92759-1

3. BHCA. (2009) Cholesterol fact sheet.

http://www.betterhealth.vic.gov.au/bhcv2/bhcarticles.nsf/pages/Cholesterol explained (Accessed September 2020)

4. Food and Agricultural Organization of the United Nations. Food based dietary guidelines by country. (2009). http://www.fao.org/ag/humannutrition/nutritioneducation/fbdg/en/ (Accessed September 2020)

5. Krauss RM, Eckel RH, Howard B, Appel LJ, Daniels SR, Deckelbaum RJ, Erdman JW Jr, KrisEtherton P, Goldberg IJ, Kotchen TA, Lichtenstein AH, Mitch WE, Mullis R, Robinson K, Wylie-Rosett J, St Jeor S, Suttie J, Tribble DL, Bazzarre TL (2000). AHA Dietary Guidelines: revision 2000: A statement for healthcare professionals from the Nutrition Committee of the American Heart Association. Circulation, 102(18), 2284-2299.

https://doi.org/10.1161/01.cir.102.18.2284

6. https://health.gov/dietaryguidelines/2015-scientific-report/pdfs/scientific-report-of-the-2015dietary-guidelines-advisory-committee.pdf (Accessed September 2020)

7. Mediterranean Diet Foundation (2010) What's the Mediterranean diet? Internet: http://dietamediterran ea.com/en/nutrition. (Accessed September 2020)

8. https://www.crea.gov.it/web/alimenti-e-nutrizione/-/linee-guida-per-una-sana-alimentazione$\underline{2018}$ (Accessed September 2020)

9. https://www.nhs.uk/live-well/eat-well/eggs-nutrition/ (Accessed September 2020) 
10. Zazpe I, Beunza JJ, Bes-Rastrollo M, Warnberg J, de la Fuente-Arrillaga C, Benito S, Vázquez Z, Martínez-González MA; SUN Project Investigators (2011). Egg consumption and risk of cardiovascular disease in the SUN Project. European journal of clinical nutrition, 65(6), 676682. https://doi.org/10.1038/ejen.2011.30

11. Qin C, Lv J, Guo Y, Bian Z, Si J, Yang L, Chen Y, Zhou Y, Zhang H, Liu J, Chen J, Chen Z, Yu C, Li L (2018). Associations of egg consumption with cardiovascular disease in a cohort study of 0.5 million Chinese adults. Heart (British Cardiac Society), 104(21), 1756-1763. https://doi.org/10.1136/heartjnl-2017-312651

12. Li Y, Zhou C, Zhou X, \& Li L. (2013). Egg consumption and risk of cardiovascular diseases and diabetes: a meta-analysis. Atherosclerosis, 229(2), 524-530.

https://doi.org/10.1016/j.atherosclerosis.2013.04.003

13. Rong Y, Chen L, Zhu T, Song Y, Yu M, Shan Z, Sands A, Hu FB, Liu L. (2013). Egg consumption and risk of coronary heart disease and stroke: dose-response meta-analysis of prospective cohort studies. BMJ (Clinical research ed.), 346, e8539.

https://doi.org/10.1136/bmj.e8539

14. Shin JY, Xun P, Nakamura Y, He K. (2013). Egg consumption in relation to risk of cardiovascular disease and diabetes: a systematic review and meta-analysis. The American journal of clinical nutrition, 98(1), 146-159. https://doi.org/10.3945/ajcn.112.051318

15. Xu L, Lam TH, Jiang CQ, Zhang WS, Zhu F, Jin YL, Woo J, Cheng KK, Thomas GN. (2019). Egg consumption and the risk of cardiovascular disease and all-cause mortality: Guangzhou Biobank Cohort Study and meta-analyses. European journal of nutrition, 58(2), 785-796. https://doi.org/10.1007/s00394-018-1692-3

16. Marventano S, Godos J, Tieri M, Ghelfi F, Titta L, Lafranconi A, Gambera A, Alonzo E, Sciacca S, Buscemi S, Ray S, Del Rio D, Galvano F, Grosso G. (2020). Egg consumption and human 
health: an umbrella review of observational studies. International journal of food sciences and nutrition, 71(3), 325-331. https://doi.org/10.1080/09637486.2019.1648388

17. Nakamura Y, Okamura T, Kita Y, Okuda N, Kadota A, Miura K, Okayama A, Ueshima H; NIPPON DATA90 Research Group (2018). Re-evaluation of the associations of egg intake with serum total cholesterol and cause-specific and total mortality in Japanese women. European journal of clinical nutrition, 72(6), 841-847. https://doi.org/10.1038/s41430-017-0051-4

18. Farvid MS, Malekshah AF, Pourshams A, Poustchi H, Sepanlou SG, Sharafkhah M, Khoshnia M, Farvid M, Abnet CC, Kamangar F, Dawsey SM, Brennan P, Pharoah PD, Boffetta P, Willett WC, Malekzadeh R. (2017). Dietary Protein Sources and All-Cause and Cause-Specific Mortality: The Golestan Cohort Study in Iran. American journal of preventive medicine, 52(2), 237-248. https://doi.org/10.1016/j.amepre.2016.10.041

19. Dehghan M, Mente A, Rangarajan S, Mohan V, Lear S, Swaminathan S, Wielgosz A, Seron P, Avezum A, Lopez-Jaramillo P, et al (2020). Association of egg intake with blood lipids, cardiovascular disease, and mortality in 177,000 people in 50 countries. The American journal of clinical nutrition, 111(4), 795-803. https://doi.org/10.1093/ajcn/nqz348

20. Zamora-Ros R, Cayssials V, Cleries R, Redondo ML, Sánchez MJ, Rodríguez-Barranco M, Sánchez-Cruz JJ, Mokoroa O, Gil L, Amiano P, Navarro C, Chirlaque MD, Huerta JM, Barricarte A, Ardanaz E, Moreno-Iribas C, Agudo A. (2019). Moderate egg consumption and allcause and specific-cause mortality in the Spanish European Prospective into Cancer and Nutrition (EPIC-Spain) study. European journal of nutrition, 58(5), 2003-2010. https://doi.org/10.1007/s00394-018-1754-6

21. Trichopoulou A, Psaltopoulou T, Orfanos P, \& Trichopoulos D. (2006). Diet and physical activity in relation to overall mortality amongst adult diabetics in a general population cohort. Journal of internal medicine, 259(6), 583-591. https://doi.org/10.1111/j.1365-2796.2006.01638.x 
22. Di Castelnuovo A, Costanzo S, Persichillo M, Olivieri M, de Curtis A, Zito F, Donati MB, de Gaetano G, Iacoviello L; MOLI-SANI Project Investigators (2012). Distribution of short and lifetime risks for cardiovascular disease in Italians. European journal of preventive cardiology, 19(4), 723-730. https://doi.org/10.1177/1741826711410820

23. Pisani P, Faggiano F, Krogh V, Palli D, Vineis P, \& Berrino F. (1997). Relative validity and reproducibility of a food frequency dietary questionnaire for use in the Italian EPIC centres. International journal of epidemiology, 26 Suppl 1, S152-S160. https://doi.org/10.1093/ije/26.suppl 1.s152

24. Salvini S, Parpinel M, Gnagnarella P, Maissoneuve P, Turrini A (1998). Banca dati composizione degli alimenti per studi epidemiologici in Italia. Milano: European Institute of Oncology

25. Pala V, Sieri S, Palli D, Salvini S, Berrino F, Bellegotti M, Frasca G, Tumino R, Sacerdote C, Fiorini L, Celentano E, Galasso R, Krogh V. (2003). Diet in the Italian EPIC cohorts: presentation of data and methodological issues. Tumori, 89(6), 594-607.

26. Trichopoulou A, Costacou T, Bamia C, Trichopoulos D (2003). Adherence to a Mediterranean diet and survival in a Greek population. N Engl J Med 348:2599e608. https://doi.org/10.1056/NEJMoa025039

27. ISTAT: Istituto Nazionale di Statistica, Atlante statistico dei comuni. Edizione 2014. Available at: https://www.istat.it/it/archivio/113712 (Accessed September 2020).

28. Hertzmark E, Pazaris M, Spiegelman D (2012). The SAS MEDIATE Macro. Boston: Harvard T.H. Chan School of Public Health.

29. http://www.bda-ieo.it/wordpress/en/ (Accessed September 2020).

30. Tong TYN, Appleby PN, Key TJ, Dahm CC, Overvad K, Olsen A, Tjønneland A, Katzke V, Kühn T, Boeing H et al. (2020). The associations of major foods and fibre with risks of ischaemic and haemorrhagic stroke: a prospective study of 418329 participants in the EPIC 
cohort across nine European countries. European heart journal, 41(28), 2632-2640. https://doi.org/10.1093/eurheartj/ehaa007

31. Zhong VW, Van Horn L, Cornelis MC, Wilkins JT, Ning H, Carnethon MR, Greenland P, Mentz RJ, Tucker KL, Zhao L, Norwood AF, Lloyd-Jones DM, Allen NB (2019). Associations of Dietary Cholesterol or Egg Consumption With Incident Cardiovascular Disease and Mortality. JAMA, 321(11), 1081-1095. https://doi.org/10.1001/jama.2019.1572

32. Djoussé L, Gaziano J M. (2008). Egg consumption in relation to cardiovascular disease and mortality: the Physicians' Health Study. The American journal of clinical nutrition, 87(4), 964 969. https://doi.org/10.1093/ajcn/87.4.964

33. Xia PF, Pan XF, Chen C, Wang Y, Ye Y, Pan A. (2020). Dietary Intakes of Eggs and Cholesterol in Relation to All-Cause and Heart Disease Mortality: A Prospective Cohort Study. Journal of the American Heart Association, 9(10), e015743. https://doi.org/10.1161/JAHA.119.015743

34. Drouin-Chartier JP, Chen S, Li Y, Schwab AL, Stampfer MJ, Sacks FM, Rosner B, Willett WC, Hu FB, Bhupathiraju SN. (2020). Egg consumption and risk of cardiovascular disease: three large prospective US cohort studies, systematic review, and updated meta-analysis. BMJ (Clinical research ed.), 368, m513. https://doi.org/10.1136/bmj.m513

35. Godos J, Micek A, Brzostek T, Toledo E, Iacoviello L, Astrup A, Franco OH, Galvano F, Martinez-Gonzalez MA, Grosso G. (2020). Egg consumption and cardiovascular risk: a doseresponse meta-analysis of prospective cohort studies. European journal of nutrition, 10.1007/s00394-020-02345-7. Advance online publication. https://doi.org/10.1007/s00394-020$\underline{02345-7}$

36. Keum N, Lee DH, Marchand N, Oh H, Liu H, Aune D, Greenwood DC, Giovannucci EL. (2015). Egg intake and cancers of the breast, ovary and prostate: a dose-response meta-analysis of prospective observational studies. The British journal of nutrition, 114(7), 1099-1107. https://doi.org/10.1017/S0007114515002135 
37. Soliman G A. (2018). Dietary Cholesterol and the Lack of Evidence in Cardiovascular Disease. Nutrients, 10(6), 780. https://doi.org/10.3390/nu10060780

38. Lecerf J M, de Lorgeril M. (2011). Dietary cholesterol: from physiology to cardiovascular risk. The British journal of nutrition, 106(1), 6-14. https://doi.org/10.1017/S0007114511000237

39. Wu JH, Zheng M, Catterall E, Downs S, Thomas B, Veerman L, Barendregt JJ. (2017). Contribution of Trans-Fatty Acid Intake to Coronary Heart Disease Burden in Australia: A Modelling Study. Nutrients, 9(1), 77. https://doi.org/10.3390/nu9010077

40. Tharrey M, Mariotti F, Mashchak A, Barbillon P, Delattre M, Fraser GE. (2018). Patterns of plant and animal protein intake are strongly associated with cardiovascular mortality: the Adventist Health Study-2 cohort. International journal of epidemiology, 47(5), 1603-1612. https://doi.org/10.1093/ije/dyy030

41. Bonaccio M, Gianfagna F, de Gaetano G, Iacoviello L. (2019). Too many individuals are unaware of their blood lipid levels, but might still get health benefit from the Mediterranean diet through lipid-independent mechanisms. European journal of preventive cardiology, 26(18), 1953-1956. https://doi.org/10.1177/2047487319867782.

42. Tsoupras A, Lordan R, Zabetakis I. (2018). Inflammation, not Cholesterol, Is a Cause of Chronic Disease. Nutrients, 10(5), 604. https://doi.org/10.3390/nu10050604

43. Giampaoli, S., Palmieri, L., Donfrancesco, C., Panico, S., Pilotto, L., Addis, A., Boccanelli, A., Di Pasquale, G., Brignoli, O., Filippi, A., Vanuzzo, D., \& Gruppo di Ricerca dell'Osservatorio Epidemiologico Cardiovascolare (2006). La valutazione del rischio cardiovascolare globale assoluto: confronto tra carta e punteggio del Progetto CUORE [Assessment of the absolute global cardiovascular risk: comparison between the risk chart and the individual score of the CUORE Project]. Giornale italiano di cardiologia (2006), 7(5), 359-364. https://doi.org/10.2427/5885 


\section{Legend to Figure 1}

Multivariable adjusted Kaplan-Meier estimates for all-cause (1A) and cardiovascular mortality (1B) for increasing number of weekly egg consumption in the Moli-sani Study cohort.

Estimates were obtained from the multivariable-adjusted model including age, sex, energy intake, educational level, residence, smoking, leisure-time PA, BMI, presence at baseline of diabetes, hyperlipidaemia, hypertension, Mediterranean diet score. 
Table 1. Baseline characteristics by categories of egg consumption in the Moli-sani Study cohort $(n=20,564)$

Egg consumption (n of egg/week)

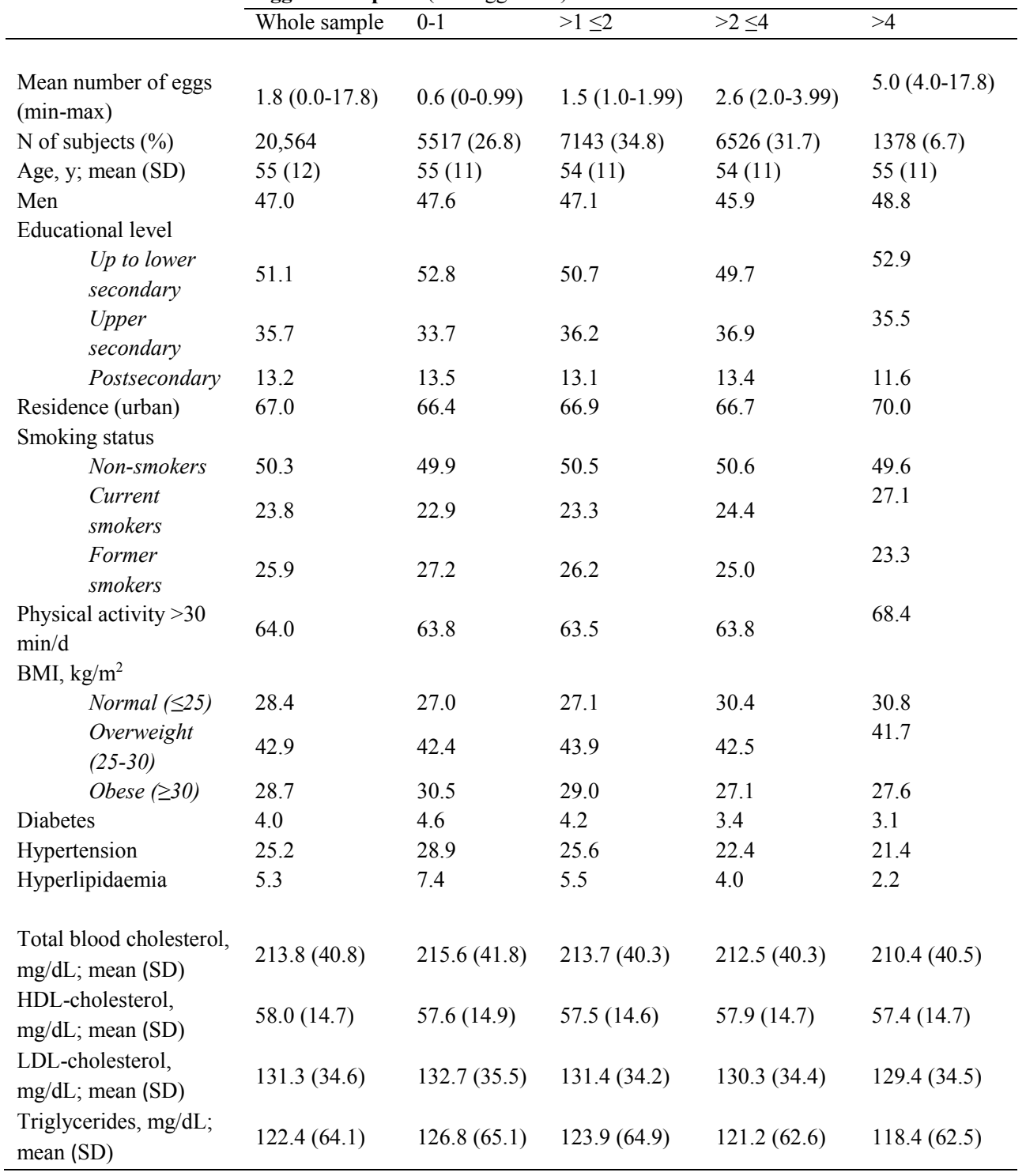

1 egg $=50$ grams.

Values are reported as percentages unless otherwise stated.

Means are adjusted for age, sex and energy intake.

Analyses for serum lipids were run on 20,148 participants. 


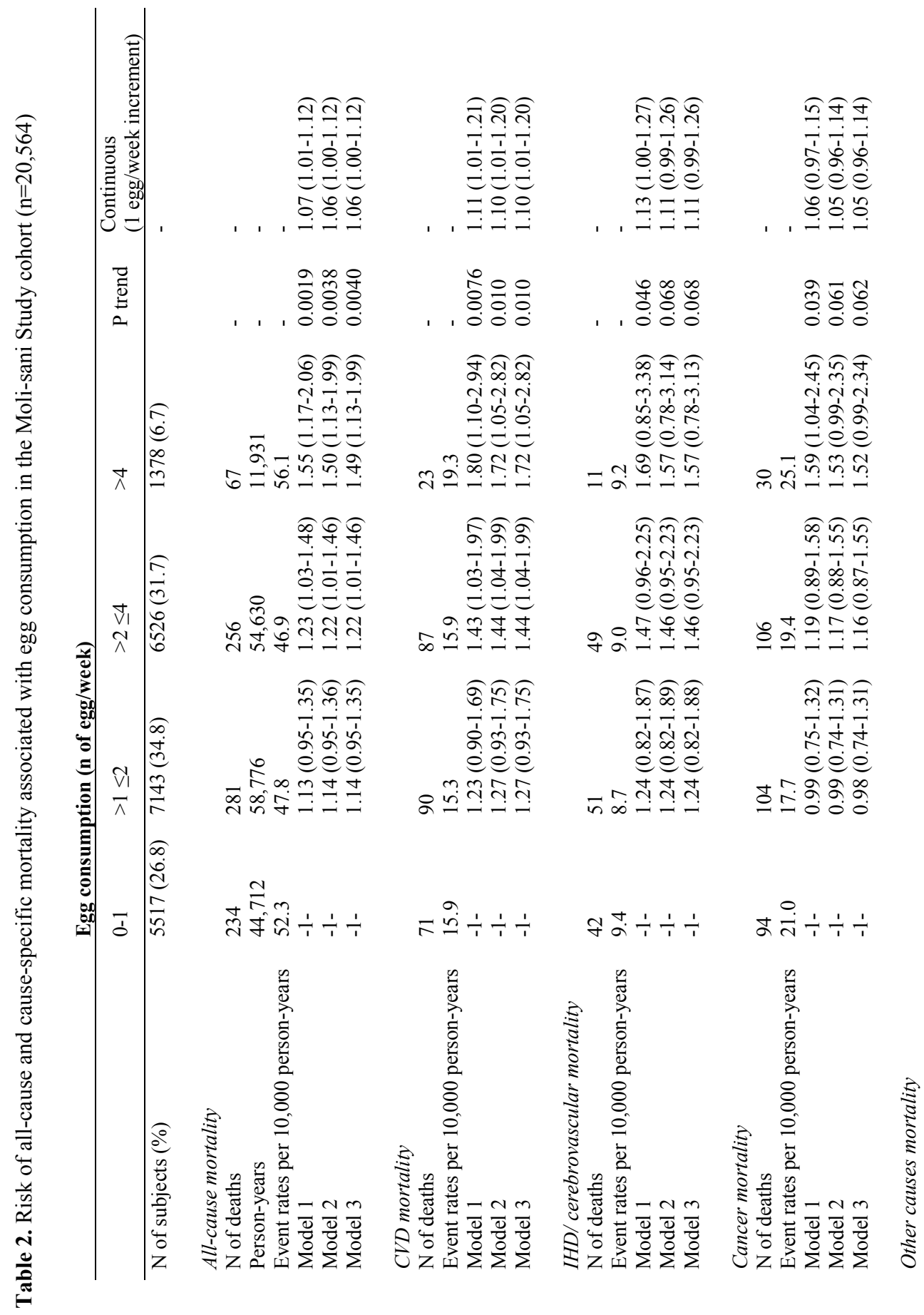




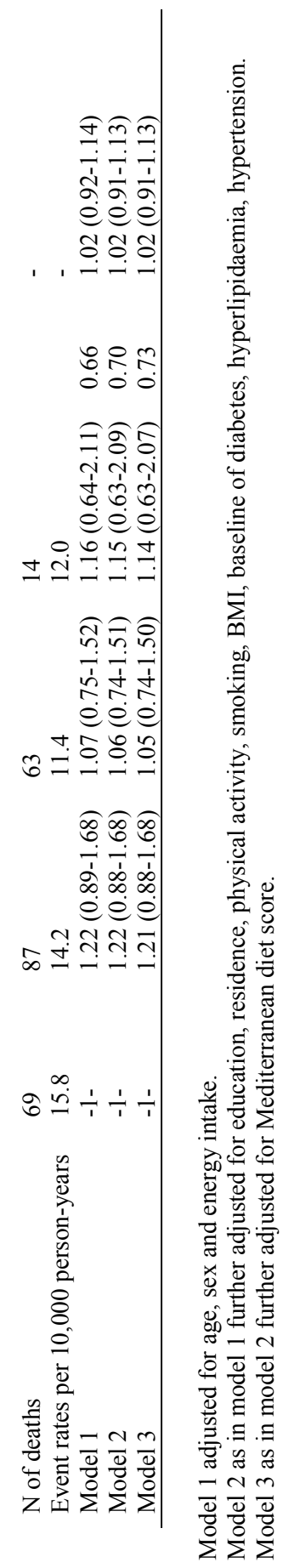




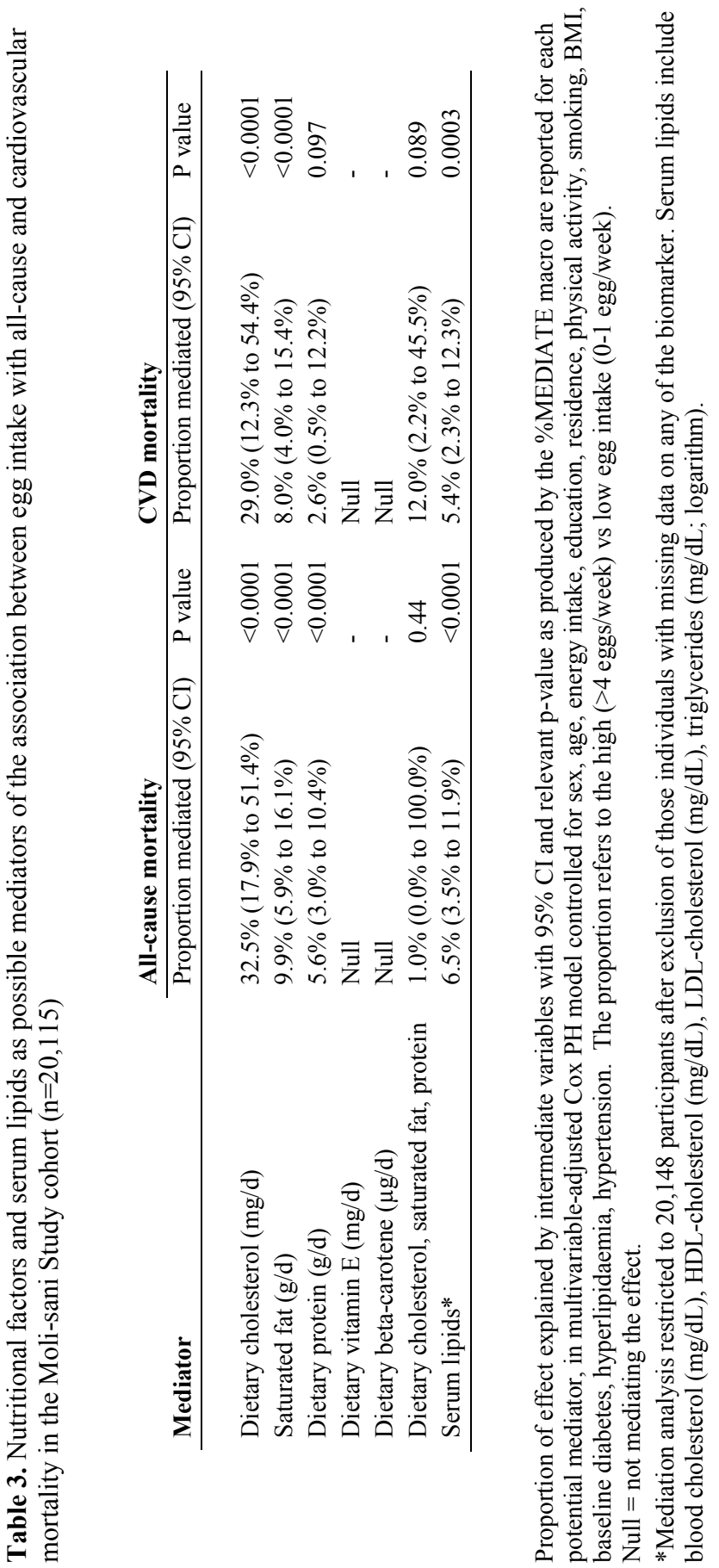



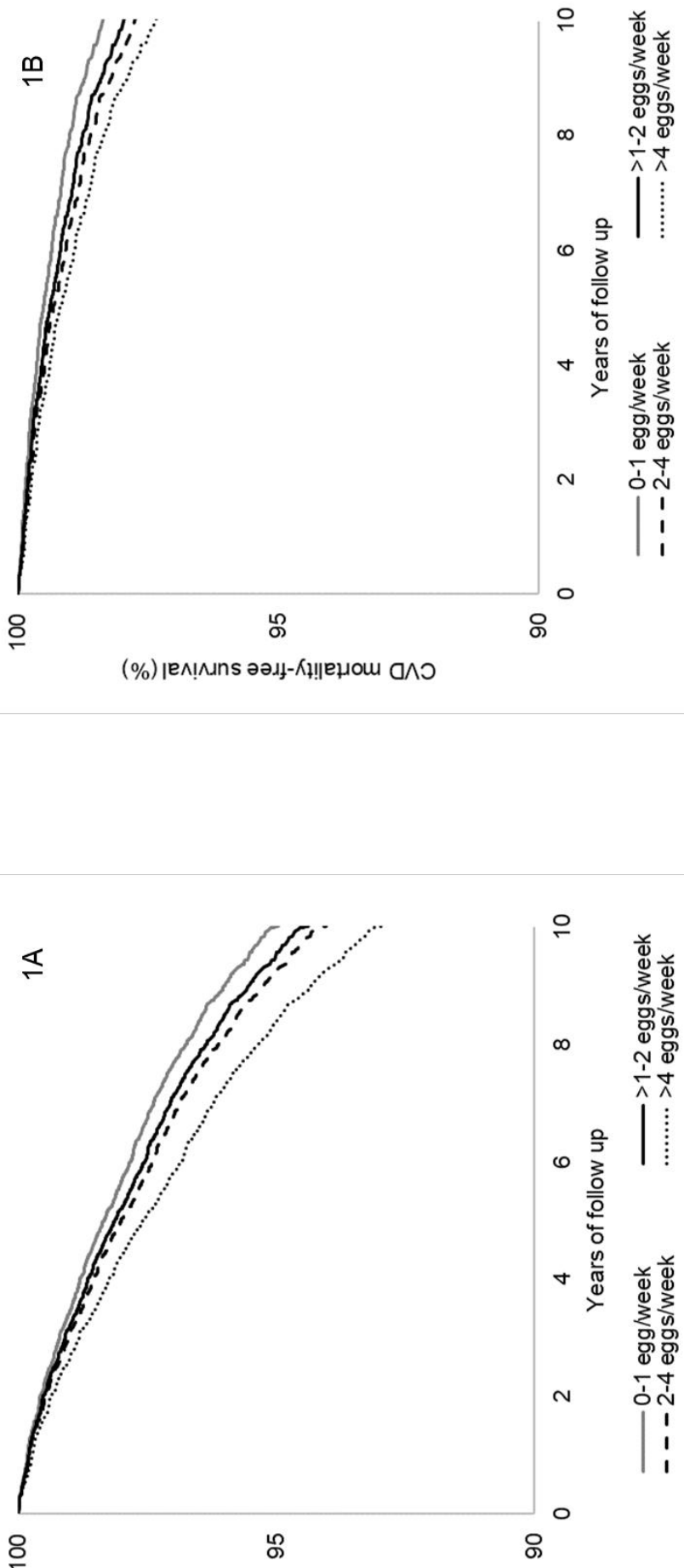

(\%) Kł!!!qeqoud jes!ıuns 

CHAPTER 7.

Chili Pepper Consumption and Mortality in Italian Adults

Bonaccio M, Di Castelnuovo A, Costanzo S, Ruggiero E, De Curtis A, Persichillo M, Tabolacci C, Facchiano F, Cerletti C, Donati MB, de Gaetano G, Iacoviello L; Moli-sani Study Investigators

J Am Coll Cardiol. 2019 Dec 24;74(25):3139-3149. 



\section{Chili Pepper Consumption and Mortality in Italian Adults}

Marialaura Bonaccio, PHD, ${ }^{\text {a }}$ Augusto Di Castelnuovo, PHD, ${ }^{\mathrm{b}}$ Simona Costanzo, PHD, ${ }^{\mathrm{a}}$ Emilia Ruggiero, MSc, ${ }^{\mathrm{a}}$ Amalia De Curtis, BSc, ${ }^{a}$ Mariarosaria Persichillo, MSc, ${ }^{a}$ Claudio Tabolacci, PHD, ${ }^{c}$ Francesco Facchiano, MD, PHD, Chiara Cerletti, PHD, ${ }^{a}$ Maria Benedetta Donati, MD, PHD, ${ }^{a}$ Giovanni de Gaetano, MD, PHD, ${ }^{a}$

Licia Iacoviello, MD, $\mathrm{PHD}$, ${ }^{\mathrm{a}, \mathrm{d}}$ on behalf of the Moli-sani Study Investigators

\section{ABSTRACT}

BACKGROUND Chili pepper is a usual part of a traditional Mediterranean diet. Yet epidemiological data on the association between chili pepper intake and mortality risk are scarce, with a lack of studies from Mediterranean populations.

OBJECTIVES This study sought to examine the association between chili pepper consumption and risk of death in a large sample of the adult Italian general population, and to account for biological mediators of the association.

METHODS Longitudinal analysis was performed on 22,811 men and women enrolled in the Moli-sani Study cohort (2005 to 2010). Chili pepper intake was estimated by the EPIC (European Prospective Investigation Into Cancer) Food Frequency Questionnaire and categorized as none/rare consumption, up to 2 times/week, $>2$ to $\leq 4$ times/week, and $>4$ times/week.

RESULTS Over a median follow-up of 8.2 years, a total of 1,236 deaths were ascertained. Multivariable hazard ratios (HRs) for all-cause and cardiovascular disease (CVD) mortality among participants in the regular ( $>4$ times/week) relative to none/rare intake were 0.77 (95\% confidence interval $[\mathrm{Cl}]: 0.66$ to 0.90$)$ and $0.66(95 \% \mathrm{Cl}: 0.50$ to 0.86$)$, respectively. Regular intake was also inversely associated with ischemic heart disease (HR: $0.56 ; 95 \% \mathrm{Cl}: 0.35$ to 0.87 ) and cerebrovascular (HR: $0.39 ; 95 \% \mathrm{Cl}: 0.20$ to 0.75 ) death risks. The association of chili pepper consumption with total mortality appeared to be stronger in hypertension-free individuals ( $p$ for interaction $=0.021$ ). Among known biomarkers of CVD, only serum vitamin D marginally accounted for such associations.

CONCLUSIONS In a large adult Mediterranean population, regular consumption of chili pepper is associated with a lower risk of total and CVD death independent of CVD risk factors or adherence to a Mediterranean diet. Known biomarkers of CVD risk only marginally mediate the association of chili pepper intake with mortality. (J Am Coll Cardiol 2019;74:3139-49) @ 2019 by the American College of Cardiology Foundation.

\footnotetext{
From the ${ }^{a}$ Department of Epidemiology and Prevention, IRCCS Neuromed, Via dell'Elettronica, Pozzilli, Italy; ${ }^{\mathrm{b}}$ Mediterranea Cardiocentro, Napoli, Italy; 'Department of Oncology and Molecular Medicine, Istituto Superiore di Sanità, Roma, Italy; and the ${ }^{\mathrm{d}}$ Department of Medicine and Surgery, Research Center in Epidemiology and Preventive Medicine (EPIMED), University of Insubria, Varese, Italy. The enrollment phase of the Moli-sani Study was supported by research grants from Pfizer Foundation (Rome, Italy), the Italian Ministry of University and Research (MIUR, Rome, Italy)-Programma Triennale di Ricerca, Decreto no. 1588, and Instrumentation Laboratory, Milan, Italy. Drs. Bonaccio and Tabolacci were supported by a Fondazione Umberto Veronesi Fellowship. Dr. Costanzo was the recipient of a Fondazione Umberto Veronesi travel grant. The present analyses were partially supported by a grant to Dr. Bonaccio from the Italian Ministry of Health 2013 grant number GR-2013-02356060, a grant to Dr. Iacoviello from the Italian Association for Cancer Research (AIRC) (AIRC “5xMILLE” n. 12237), and a grant to Dr. Iacoviello as a partner of BiomarCaRE (Biomarkers for Cardiovascular Risk Assessment in Europe) by the European Commission Seventh Framework Programme FP7/2007-2013 (HEALTH-F2-2011-278913). The funders had no role in study design; collection, analysis, and interpretation of data; writing of the manuscript; or the decision to submit the article for publication. The authors have reported that they have no relationships relevant to the contents of this paper to disclose.
} 


A B B R E V I A T I O N S
A N D A C R O N Y M S
BMI = body mass index
CI = confidence interval
CRP = C-reactive protein
CV = coefficient of variability
CVD = cardiovascular disease
HDL = high-density lipoprotein
HR = hazard ratio
ICD-9 = International
Classification of Diseases-
9th Revision
IHD = ischemic heart disease
MD = Mediterranean diet
MDS = Mediterranean Diet
Score
NRI = Net Reclassification
Index
NT-proBNP = N-terminal pro-
B-type natriuretic peptide
PA = physical activity

S pices have long been an essential part of the traditional Mediterranean diet (MD), and are placed, along with herbs, at the base of the MD pyramid both for their nutritional properties and as a valuable substitute for salt (1-3). Chili peppers, belonging to the genus Capsicum, are native to Central and Southern America, but are largely present in different cultures' diets worldwide (4), and are used to flavor traditional dishes from the southern Italian regions.

\section{SEE PAGE 3150}

Health benefits of chili peppers have been ascribed to capsaicin, its major pungent compound, which has been observed to favorably improve cardiovascular function and metabolic regulation in experimental and population studies (5). In addition to its anti-inflammatory and analgesic properties and atheroprotective effects (4), capsaicin reportedly induces apoptosis of the tumor cells (6); high concentrations, however, likely evoke deleterious effects (7).

Studies in humans concluded that chili pepper intake facilitates weight loss through activation of different receptors and improved control of insulin (8). Evidence from a large epidemiological study appears to corroborate the weight-loss properties of chili peppers by showing inverse associations with incidence of overweight/obesity (9).

More recently, animal studies uncovered an interaction between dietary capsaicin and gut microbiota as a novel mechanism for the antiobesity effect of capsaicin acting through prevention of microbial dysbiosis, gut barrier dysfunction, and chronic lowgrade inflammation (10). To date, epidemiological evidence longitudinally addressing the association of chili pepper intake on disease/mortality risk is scarce.

With the exception of 2 population studies from China (11) and the United States (12), both showing lower mortality risk associated with regular chili pepper intake, no epidemiological investigation on the potential health benefits associated with consumption of chili pepper is currently available from European cohorts or from Mediterranean areas. In addition, none of the aforementioned studies has addressed possible biological mechanisms through which regular chili pepper intake may provide the observed health advantages.

The primary aim of this study was to prospectively estimate the association of chili pepper consumption with total and cause-specific mortality in a large
Mediterranean population of adult men and women; as a secondary purpose, we examined some biological mechanisms that could be on the pathway between chili pepper intake and mortality by analyzing the possible contribution of established biomarkers of cardiovascular risk. Finally, we assessed whether inclusion of chili pepper was able to improve risk prediction associated with a traditional Mediterranean Diet Score (MDS).

\section{METHODS}

STUDY POPULATION. We used data from the Molisani Study, a prospective cohort study established in 2005 to 2010 with an enrollment of 24,325 men and women ( $\geq 35$ years of age) randomly recruited from the general population of Molise, a southern Mediterranean region in Italy, with the main purpose of investigating genetic and environmental risk factors of cardiovascular, cerebrovascular, and tumor diseases. Details of the Moli-sani Study have been previously described (13).

Participants reporting implausible energy intakes $(<800 \mathrm{kcal} / \mathrm{day}$ in men and $<500 \mathrm{kcal} /$ day in women or $>4,000 \mathrm{kcal} /$ day in men and $>3,500 \mathrm{kcal} /$ day in women; $3.2 \%$ ) were excluded, as well as individuals with unreliable medical/dietary questionnaires $(1 \%$ and $3.9 \%$, respectively), individuals with missing information on main covariates (0.3\%), exposure (0.4\%), and cause-specific mortality (0.2\%), and 23 participants $(0.1 \%)$ lost to follow-up. The final sample consisted of 22,811 subjects.

The cohort was followed up for mortality until December 31, 2015. Overall and cause-specific mortality was assessed by the Italian mortality registry (ReNCaM registry), validated by Italian death certificates (ISTAT form) and coded according to the International Classification of Diseases-9th Revision (ICD-9).

Cardiovascular disease (CVD) mortality included deaths from diseases of the circulatory system, when the underlying cause of death included ICD-9 codes 390 to 459 . ICD-9 codes 430 to 438 were used to define specific cause of death for cerebrovascular disease, and ICD-9 codes 410 to 414 and 429 for ischemic heart disease (IHD). Cancer death was considered when the underlying cause of death included ICD-9 codes 140 to 208.

Noncardiovascular/noncancer causes of death were included in the "other-cause mortality" group. The Moli-sani Study complies with the Declaration of Helsinki and was approved by the ethical committee of the Catholic University Medical School in Rome, Italy. All participants provided written informed consent. 
COVARIATE ASSESSMENT. History of cardiovascular disease (angina, myocardial infarction, revascularization procedures, peripheral artery diseases, and cerebrovascular events) was self-reported and confirmed by medical records and therapy. History of cancer was self-reported and confirmed by medical records. Hypertension, hyperlipidemia, and diabetes were defined by use of pharmacological treatment.

Leisure-time physical activity (PA) was expressed as daily energy expenditure in metabolic equivalent task-hours/day for sport, walking, and gardening. Height and weight were measured, and body mass index (BMI) was calculated as $\mathrm{kg} / \mathrm{m}^{2}$ and then grouped into 3 categories as normal $(\leq 25)$, overweight ( $>25$ to $<30$ ), or obese $(\geq 30)$.

Subjects were classified as never-smokers, current smokers, or ex-smokers (quit at least 1 year ago). Education was based on the highest qualification attained and was categorized as up to lower secondary (approximately $\leq 8$ years of study), upper secondary school ( $>8$ to $\leq 13$ years), and postsecondary education ( $>13$ years).

Occupational social class was based on the Registrar General's occupation-based classification scheme and ranked as previously described within the Molisani population (14), and categorized as professional/managerial, skilled nonmanual occupations, skilled manual occupations, partly skilled occupations, and unskilled, retired/housewife, unemployed/ unclassified subjects.

DIETARY ASSESSMENT. Food intake during the year before enrollment was assessed by the EPIC (European Prospective Investigation Into Cancer) Food Frequency Questionnaire validated and adapted to the Italian population (15). Frequency of chili pepper intake was estimated through the question: "How often do you consume foods containing chili pepper," with possible answers being "never or almost never," "occasionally," "often," and "very often."

Chili pepper was then categorized as consumption on a weekly basis that is never/rare intake, up to 2 times/week, $>2$ to $\leq 4$ times/week, and $>4$ times/ week. Other herbs and spices including garlic, parsley, and black pepper were categorized as yes/no.

Adherence to the traditional MD was assessed through the MDS developed by Trichopoulou et al. (16), and was obtained by assigning 1 point to healthy foods (fruits and nuts, vegetables, legumes, fish, cereals, monounsaturated-to-saturated fats ratio) whose consumption was above the sex-specific medians of intake of the Moli-sani Study population, free from CVD, cancer, and diabetes; foods presumed to be detrimental (meat and dairy products) were scored positively if their consumption was below the median. All other intakes received o points. For ethanol, men who consumed 10 to $50 \mathrm{~g} /$ day and women who consumed 5 to $25 \mathrm{~g}$ /day received 1 point; otherwise, the score was 0 . The MDS ranged from 0 to 9 (the latter reflecting maximal adherence). The vegetable food group included in the MDS did not include sweet peppers; the latter were used as a covariate in survival analysis and also independently tested as predictors of mortality.

Food antioxidant content was appraised by a score determining the content in antioxidant vitamins and phytochemicals of each food group, and ranged from -99 to 99 , with higher values indicating increased consumption of foods rich in antioxidants (17). The polyphenol content of diet was measured by a polyphenol antioxidant content score calculated as in Pounis et al. (18).

Variety of fruit and/or vegetable intake was assessed by 4 different (fruit, vegetables, vegetable subgroups, and fruit/vegetables combined) Diet Diversity Scores, following similar approaches tested within EPIC cohorts (19). Diversity was intended as the total number of individual vegetable/fruit products eaten at least once in 2 weeks.

BIOMARKERS OF CVD RISK. Antecubital venous blood samples were obtained from participants who had fasted overnight and had refrained from smoking for at least $6 \mathrm{~h}$. Serum lipids (total cholesterol, highdensity lipoprotein [HDL]-cholesterol, triglycerides) and blood glucose were assayed by enzymatic reaction methods using an automatic analyzer (ILab 350; Instrumentation Laboratory, Milan, Italy).

Quality control for lipids and glucose was obtained by a commercial standard (Ser 1 and Ser 2) provided by Instrumentation Laboratory and an in-house serum standard pool. The coefficients of variability (CVs) were respectively $4.9 \%, 5.2 \%$, and $4 \%$ for blood cholesterol; $3.2 \%$, $3 \%$, and $4.5 \%$ for HDL-cholesterol; $5.2 \%, 5.3 \%$, and $5 \%$ for triglycerides; and $4.7 \%$, $4.1 \%$, and 3.9 for blood glucose.

High-sensitivity C-reactive protein (CRP) was measured in fresh serum samples by a particleenhanced immunoturbidimetric assay (ILab 350). Quality control for CRP was maintained using an inhouse serum pool and internal laboratory standard at $1.5 \mathrm{mg} / \mathrm{l}$; interday CVs for CRP were $5.5 \%$ and $4.2 \%$, respectively. Hemochromocytometric analysis was performed by cell count (Coulter HMX; Beckman Coulter, Milan, Italy) within $3 \mathrm{~h}$ from blood collection. Quality control was performed by using 3 different levels of standards Abn (abnormal) I, Abn II, and Normal (Coulter HMX). The CV for white blood 


\begin{tabular}{|c|c|c|c|c|c|}
\hline & \multicolumn{5}{|c|}{ Chili Pepper Intake (Times/Week) } \\
\hline & $\begin{array}{c}\text { None/Rare } \\
(\mathrm{n}=7,689,33.7 \%)\end{array}$ & $\begin{array}{c}\text { Up to } 2 \\
(n=4,360,19.1 \%)\end{array}$ & $\begin{array}{c}>2 \text { to } \leq 4 \\
\text { (n }=5,216,22.9 \%)\end{array}$ & $\begin{array}{c}>4 \\
(n=5,546,24.3 \%)\end{array}$ & p Value \\
\hline Times/week & 0.0 & $0.8 \pm 0.4$ & $2.8 \pm 0.6$ & $7.0 \pm 3.8$ & - \\
\hline Age, yrs & $55 \pm 13$ & $54 \pm 11$ & $55 \pm 11$ & $56 \pm 11$ & $<0.0001$ \\
\hline Male & 34.6 & 50.7 & 52.5 & 58.8 & $<0.0001$ \\
\hline Post-secondary education & 11.5 & 14.1 & 13.4 & 13.5 & $<0.0001$ \\
\hline Professional/managerial workers & 18.7 & 21.3 & 21.8 & 21.6 & $<0.0001$ \\
\hline Smokers & 18.6 & 22.0 & 24.8 & 28.1 & 0.0022 \\
\hline Leisure-time PA, MET-h/day & $3.4 \pm 3.8$ & $3.3 \pm 3.7$ & $3.7 \pm 4.0$ & $3.7 \pm 4.4$ & $<0.0001$ \\
\hline Drugs for diabetes & 4.3 & 4.6 & 4.9 & 5.5 & 0.015 \\
\hline Drugs for hypertension & 27.3 & 25.8 & 27.0 & 28.8 & 0.016 \\
\hline Lipid-lowering drugs & 7.4 & 7.0 & 7.7 & 8.5 & 0.0011 \\
\hline Cardiovascular disease & 4.8 & 5.2 & 4.8 & 6.0 & 0.010 \\
\hline Cancer & 3.9 & 3.0 & 3.1 & 2.6 & 0.0090 \\
\hline Mediterranean Diet Score* & $4.2 \pm 1.6$ & $4.3 \pm 1.6$ & $4.4 \pm 1.6$ & $4.8 \pm 1.6$ & $<0.0001$ \\
\hline Sweet pepper intake, g/day & $2.7 \pm 3.2$ & $2.8 \pm 2.9$ & $3.2 \pm 3.3$ & $3.7 \pm 3.9$ & $<0.0001$ \\
\hline Black pepper & 11.0 & 19.3 & 25.0 & 26.0 & $<0.0001$ \\
\hline Garlict & 82.6 & 85.0 & 89.3 & 92.4 & $<0.0001$ \\
\hline Parsleyt & 91.7 & 95.1 & 95.6 & 96.7 & $<0.0001$ \\
\hline \multicolumn{6}{|c|}{$\begin{array}{l}\text { Values are mean } \pm \mathrm{SD} \text { or } \% \text {, unless otherwise indicated. Means and } \mathrm{p} \text { values are adjusted for age, sex, and energy intake (Kcal/day). }{ }^{*} \text { Not including sweet pepper intake. }+ \text { Black } \\
\text { pepper, garlic, and parsley consumption was reported as prevalence of consumers. }\end{array}$} \\
\hline $\mathrm{MET}=$ met & ctivity. & & & & \\
\hline
\end{tabular}

cells was $6.2 \%, 3.3 \%$, and $3.0 \%$ for Abn I, Abn II, and Normal, respectively. N-terminal pro-B-type natriuretic peptide (NT-proBNP), high-sensitivity troponin I (hsTnI), apolipoprotein A1 (ApoA), apolipoprotein B100 (ApoB100), lipoprotein a [Lp(a)], markers of renal function (cystatin C, creatinine), insulin, C-peptide, and serum vitamin D were measured in the framework of the collaborative BiomarCaRE (Biomarker for Cardiovascular Risk Assessment across Europe) project (20).

STATISTICAL ANALYSIS. Baseline characteristics of the participants by categories of chili pepper intake were summarized and compared by using analysis of variance adjusted for age, sex, and energy intake (GENMOD procedure for categorical variables and GLM procedure for continuous variables in SAS software, version 9.4 [SAS Institute, Cary, North Carolina]) (Table 1).

Risk estimates for mortality were expressed as hazard ratios (HRs) with $95 \%$ confidence interval (CI) and calculated by using Cox regression models with time-on-study on the time scale and competing risk of dying for other causes. Multivariable-adjusted HRs were calculated across 4 categories of intake (none/ rare consumption-referent category, up to 2 times/ week, $>2$ to $\leq 4$ times/week, and $>4$ times/week) as well as considering consumers as opposed to nonconsumers.
Based on previous published reports and biological plausibility, 2 multivariable models were fitted: the first (model 1) was adjusted for age (continuous), sex, and energy intake (kcal/day; continuous); the second (model 2) as in model 1 and further controlled for educational level, occupational social class, smoking, leisure-time PA, treatment for diabetes, medication for hypertension, lipid-lowering drugs, history of CVD or cancer at baseline, MDS (continuous), and sweet pepper intake (g/day, ordered quartiles), garlic, parsley, and black pepper (yes/no).

Several panels of biomarkers were tested as possibly mediating the association of chili pepper intake with mortality risk. In addition to cardiac troponin (hsTnI) and NT-proBNP, we tested biomarkers of renal function (cystatin C, creatinine), glucose metabolism (blood glucose, insulin, C-peptide), lipid metabolism (total blood cholesterol, HDLcholesterol, triglycerides, $\mathrm{Lp}(\mathrm{a})$, apoA1, and apoB100), serum vitamin $D$, inflammatory markers (CRP and white blood cells), and a panel including systolic and diastolic blood pressure and BMI.

The multivariable model 2 served as the reference for the mediation analysis used to quantify the contribution of each set of potential mediators, which were alternately included into model 2 . For the mediation analysis, the \%MEDIATE macro in SAS (21) was used, which calculates the point and interval 
estimates of the percent of exposure effect explained by 1 or more intermediate variables, with $95 \% \mathrm{CI}$ and $\mathrm{p}$ values. Biomarkers were entered into the mediation analysis as ordered quintiles.

Subgroup analyses were performed within a healthy sample (individuals without CVD or cancer and who were not using drugs for diabetes at baseline) and excluding early deaths (follow-up $>2$ years). We tested the interaction between chili pepper and a large panel of potential effect modifiers by introducing in a Cox analysis a product term of chili pepper intake (yes vs. no) $\times$ a given effect modifier ( 2 categories, with the exception of adherence to MD, 3 categories).

Consumption of chili pepper (none/rare intake $=0$ points; regular intake $=1$ point) was included as an additional component into the original MDS, thus originating an MDS "supplemented" with chilies, ranging from 0 to 10; to allow comparison among scores, they were entered into risk analyses scaled by their standard deviation.

The area under the receiver-operating curve statistic, the categorical net reclassification index (NRI) and the integrated discrimination improvement (22) were used to quantify the predictive value of the MDS "supplemented" with chilies over the traditional MDS. To estimate these metrics, the follow-up time was censored at 10 years. The risk categories chosen for NRI calculation were $<5 \%$ and $\geq 5 \%$. Observed mortality rates over time were showed as curves obtained by using the LIFETEST procedure in SAS. Dummy variables for missing values were created. Positively skewed variables were log transformed before analysis. Subgroup and interaction analyses were run by excluding missing categories. The data analysis was generated using SAS/STAT software, Version 9.4 of the SAS System for Windows 2009.

\section{RESULTS}

Regular consumption ( $>4$ times/week) of chili pepper was reported by $24.3 \%$ of the study participants, whereas $33.7 \%$ declared none/rare intake (Table 1 ). Regular consumers were more likely to be men, slightly older, to report higher educational level and occupation, and showed higher prevalence of risk factors (diabetes, hypertension, hyperlipidemia, and history of CVD), but greater leisure-time PA (Table 1). Higher consumption of chili pepper was also associated with closer adherence to MD, higher intake of other spices (Table 1), and a global higher diet quality as measured by dietary polyphenol and antioxidant content (Online Table 1). Regular consumers also tended to have higher levels of blood lipids and blood glucose, and higher BMI, and slightly higher blood leukocyte counts, whereas no substantial differences were observed for CRP (Online Table 2). NT-proBNP levels were lower in regular consumers, whereas troponin I and serum vitamin D were more likely associated with higher chili intake.

Over a median follow-up of 8.2 years (interquartile range: 7.3 to 9.3 years; 187,584 person years), a total of 1,236 deaths were ascertained (CVD $=444$, IHD/ cerebrovascular $=258$, cancer $=482$, and 310 from other causes). In a model adjusted only for age, sex, and energy intake, regular consumption ( $>4$ times/ week) of chili pepper was associated with $23 \%$ ( $95 \%$ CI: $10 \%$ to $34 \%$ ) lower risk of all-cause mortality, as opposed to none/rare intake, and results remained substantially unchanged in the fully adjusted model (Table 2).

In comparison with individuals reporting none/ rare intake, regular consumers experienced $34 \%$ ( $14 \%$ to $50 \%$ ) lower risk of CVD mortality, whereas multivariable HRs for IHD and cerebrovascular death were 0.56 (95\% CI: 0.35 to 0.87 ) and 0.39 (95\% CI: 0.20 to 0.75 ), respectively (Table 2 ).

For all mortality outcomes under study, we failed to find a clear stepwise dose-response relation with chili pepper, indicating that the actual advantage in terms of survival improvement is likely attributable to the fact of consuming chilies rather than not consuming them (Table 2). Observed mortality rates and multivariable Kaplan-Meier estimates for consumers versus none/rare consumers are wellseparated (Central Illustration).

Cancer mortality risk was slightly, though not significantly, affected by chili pepper consumption, although a downward trend was observed (Table 2). Finally, consumption of chili pepper was inversely associated with mortality for other causes (Table 2). Sweet pepper intake was associated with lower total and IHD mortality risk, whereas no association was found with cerebrovascular mortality (Online Table 3), after adjustment for potential confounders.

Established biomarkers of CVD did not substantially modify the relation between chili pepper and mortality, although a marginal role was played by serum vitamin D levels and biomarkers of lipid metabolism, explaining $6.1 \%$ and $5.3 \%$ of the association with all-cause mortality, respectively (Table 3 ). Subgroup analyses showed no difference of effect across levels of main covariates, with the exception of hypertension, which was likely to modify the magnitude of the association between chili pepper intake and total mortality risk. Specifically, the positive effect of chilies was stronger in hypertensionfree individuals ( $\mathrm{p}$ for interaction $=0.021$ ) (Table 4). Overall diet quality, measured by MDS, was not an 


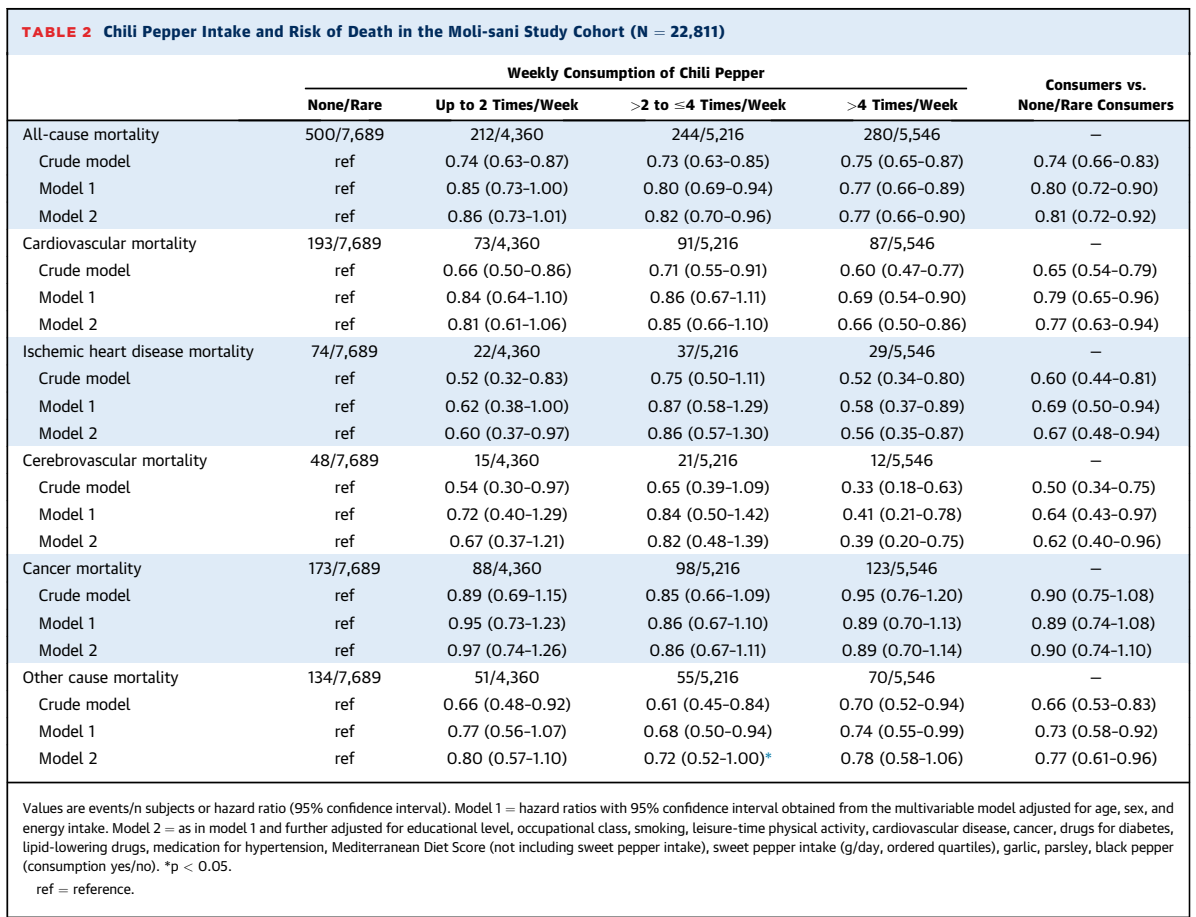

effect modifier of the relation between chili pepper and mortality risk.

Risk of CVD mortality associated with a 1 SD increment in the MDS was 0.92 (95\% CI: 0.83 to 1.02 ) and became 0.89 ( $95 \% \mathrm{CI}$ : 0.80 to 0.98 ) in association with 1 SD increment in the MDS "supplemented" with chili pepper. Similarly, risk reduction of IHD/cerebrovascular death went from $14 \%$ to $19 \%$, whereas no substantial modifications were observed for cancer and other causes of mortality (data not shown). The inclusion of chili pepper consumption as an additional component of the traditional MDS did not improve discrimination ability of the model, with the exception of cerebrovascular mortality. However, the improved prediction associated with the MDS supplemented with chilies was slightly over the model including the traditional MDS (integrated discrimination improvement $=0.005 ; \mathrm{p}=0.026$; $\mathrm{NRI}=0.010$; $\mathrm{p}=0.66$; $\mathrm{p}$ value for difference in area under the curve $=0.31)$ (Online Table 4 ).

\section{DISCUSSION}

Findings from this large Mediterranean populationbased cohort show that regular consumption of chili pepper is associated with lower risk of total and CVD mortality, with larger magnitude observed for IHD and cerebrovascular-related deaths. On the contrary, no cancer death risk reduction was found, whereas regular intakes were associated with lower risk of other causes of mortality.

Our findings are in agreement and corroborate the main results of 2 earlier studies from nonMediterranean cohorts. Evidence from the China Kadoorie Biobank on about 500,000 men and women (11) showed that regular consumption of spicy food (almost every day) lowered the risk of total mortality by $14 \%$ and of IHD death by $22 \%$; reductions were also observed for deaths due to cancer $(-8 \%)$, or respiratory diseases $(-29 \%)$. More recently, in the large NHANES (National Health and Nutrition Examination 


\section{CENTRAL ILLUSTRATION Chili Pepper and Mortality}

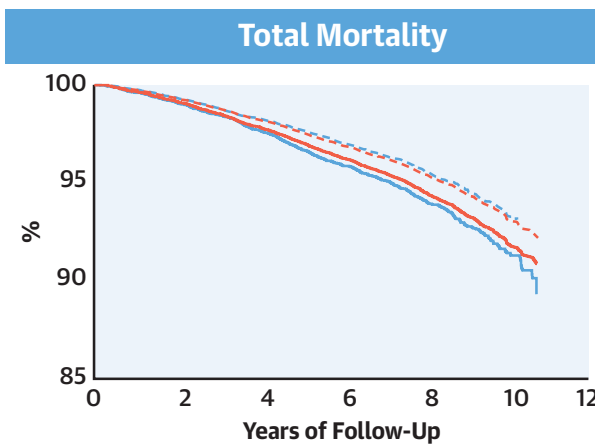

\section{Cardiovascular Disease Mortality}

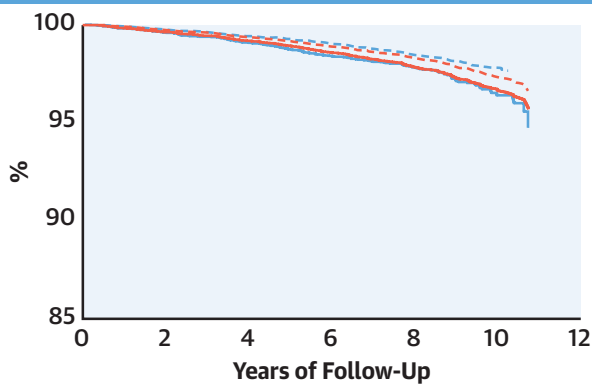

\section{Ischemic Heart Disease Mortality}

\section{Cerebrovascular Mortality}
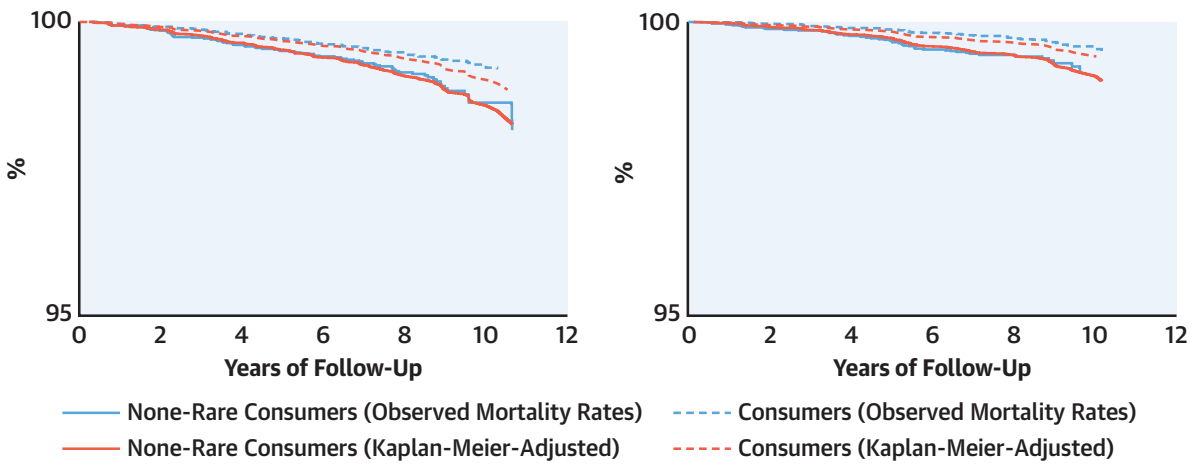

- - - - - Consumers (Observed Mortality Rates)

- - - - - Consumers (Kaplan-Meier-Adjusted)

Bonaccio, M. et al. J Am Coll Cardiol. 2019;74(25):3139-49.

Observed rates and Kaplan-Meier estimates for all-cause, cardiovascular disease, ischemic heart disease, and cerebrovascular mortality for consumers and none/rareconsumers of chili pepper in the Moli-sani Study cohort. Kaplan-Meier estimates were obtained from the multivariable model adjusted for age, sex, educational level, occupational class, smoking, leisure-time physical activity, cardiovascular disease, cancer, drugs for diabetes, lipid-lowering drugs, medication for hypertension, Mediterranean diet score (not including sweet pepper intake), sweet pepper intake ( $\mathrm{g} / \mathrm{day}$, ordered quartiles), garlic, parsley, black pepper (consumption yes/no), and energy intake (kcal/day).

Survey) cohort, the consumption of hot red chili peppers appeared to be associated with a $13 \%$ reduction in total mortality risk (12).

Our study reveals that the health benefits associated with chili pepper intake are independent of the overall quality of the diet, as measured by a traditional Mediterranean diet, thus suggesting an independent effect of chili pepper toward mortality risk. Moreover, risk estimates for total and cause-specific mortality in the minimally adjusted and fully adjusted multivariable models were very similar, suggesting that, although chili pepper consumption was associated with several risk factors for CVD, its relationship with total or cause-specific mortality was independent from such factors.

Furthermore, sensitivity analysis showed that the association between chili consumption and mortality was similar across risk factor categories, with the exception of hypertension, where the association was significantly stronger in normotensive subjects, 


\begin{tabular}{|c|c|c|c|c|}
\hline & \multicolumn{4}{|c|}{ Chili Pepper Consumption } \\
\hline & $\begin{array}{l}\text { None/Rare Consumers } \\
\quad(n=7,689)\end{array}$ & $\begin{array}{l}\text { Consumers } \\
(n=15,122)\end{array}$ & PTE (\%) & PTE $p$ Value \\
\hline All-cause mortality, deaths & $500(6.5)$ & $736(4.9)$ & - & \\
\hline Model 2 & ref & $0.81(0.72-0.92)$ & - & \\
\hline Model $2+$ cardiac troponin & ref & $0.81(0.72-0.91)$ & Null & \\
\hline Model $2+$ natriuretic peptide & ref & $0.82(0.72-0.92)$ & Null & \\
\hline Model $2+$ biomarkers of renal function & ref & $0.82(0.72-0.92)$ & Null & \\
\hline Model $2+$ biomarkers of glucose metabolism & ref & $0.81(0.72-0.91)$ & Null & \\
\hline Model $2+$ biomarkers of lipid metabolism & ref & $0.82(0.73-0.93)$ & $5.3[1.6-16.5]$ & 0.033 \\
\hline Model 2 + serum vitamin $\mathrm{D}(\mathrm{ng} / \mathrm{ml})$ & ref & $0.83(0.73-0.93)$ & $6.1[2.0-16.6]$ & 0.014 \\
\hline Model $2+$ inflammatory biomarkers & ref & $0.81(0.72-0.91)$ & Null & \\
\hline Model $2+$ BP + BMI & ref & $0.82(0.73-0.92)$ & $2.4[0.4-13.1]$ & 0.12 \\
\hline Cardiovascular mortality, deaths & $193(2.5)$ & $251(1.7)$ & - & \\
\hline Model 2 & ref & $0.77(0.63-0.94)$ & - & \\
\hline Model $2+$ cardiac troponin & ref & $0.75(0.62-0.93)$ & Null & \\
\hline Model $2+$ natriuretic peptide & ref & $0.76(0.62-0.93)$ & Null & \\
\hline Model $2+$ biomarkers of renal function & ref & $0.77(0.63-0.95)$ & $1.7[0.0-38.2]$ & 0.28 \\
\hline Model $2+$ biomarkers of glucose metabolism & ref & $0.76(0.62-0.94)$ & Null & \\
\hline Model $2+$ biomarkers of lipid metabolism & ref & $0.77(0.63-0.95)$ & $3.1[0.2-31.0]$ & 0.21 \\
\hline Model 2 + serum vitamin $D(\mathrm{ng} / \mathrm{ml})$ & ref & $0.78(0.64-0.96)$ & $5.9[1.4-21.9]$ & 0.048 \\
\hline Model $2+$ inflammatory biomarkers & ref & $0.76(0.62-0.93)$ & Null & \\
\hline Model $2+$ BP + BMI & ref & $0.77(0.63-0.94)$ & Null & \\
\hline Ischemic heart disease mortality, deaths & $74(1.0)$ & $88(0.6)$ & - & \\
\hline Model 2 & ref & $0.67(0.48-0.94)$ & - & \\
\hline Model $2+$ cardiac troponin & ref & $0.67(0.48-0.93)$ & Null & \\
\hline Model $2+$ natriuretic peptide & ref & $0.67(0.48-0.93)$ & Null & \\
\hline Model 2 + biomarkers of renal function & ref & $0.67(0.48-0.93)$ & Null & \\
\hline Model $2+$ biomarkers of glucose metabolism & ref & $0.66(0.47-0.92)$ & Null & \\
\hline Model 2 + biomarkers of lipid metabolism & ref & $0.66(0.48-0.92)$ & Null & \\
\hline Model 2 + serum vitamin $D(\mathrm{ng} / \mathrm{ml})$ & ref & $0.68(0.48-0.95)$ & $2.7[0.2-29.2]$ & 0.20 \\
\hline Model $2+$ inflammatory biomarkers & ref & $0.67(0.48-0.93)$ & Null & \\
\hline Model $2+$ BP + BMI & ref & $0.67(0.48-0.93)$ & Null & \\
\hline Cerebrovascular mortality, deaths & $48(0.6)$ & $48(0.3)$ & - & \\
\hline Model 2 & ref & $0.62(0.40-0.96)$ & - & \\
\hline Model $2+$ cardiac troponin & ref & $0.61(0.39-0.95)$ & Null & \\
\hline Model $2+$ natriuretic peptide & ref & $0.62(0.40-0.96)$ & $1.2[0.0-91.3]$ & 0.38 \\
\hline Model 2 + biomarkers of renal function & ref & $0.62(0.40-0.96)$ & Null & \\
\hline Model $2+$ biomarkers of glucose metabolism & ref & $0.62(0.40-0.96)$ & $1.0[0.0-49.8]$ & 0.33 \\
\hline Model 2 + biomarkers of lipid metabolism & ref & $0.61(0.39-0.95)$ & Null & \\
\hline Model 2 + serum vitamin $D(\mathrm{ng} / \mathrm{ml})$ & ref & $0.62(0.40-0.96)$ & Null & \\
\hline Model $2+$ inflammatory biomarkers & ref & $0.61(0.39-0.94)$ & Null & \\
\hline Model $2+$ BP + BMI & ref & $0.62(0.40-0.96)$ & Null & \\
\hline
\end{tabular}

suggesting that the high prevalence of hypertension in chili pepper consumers could partially mask its protective effect on total mortality.

With respect to a potential improvement of risk prediction deriving from the inclusion of chilies into the traditional MDS, we found no significant changes in the discrimination ability of the modified score. The fact that an explanatory variable significantly correlated with the outcome does not lead to improvements in prediction is a well-known and widespread occurrence, and does not entirely devaluate the association between the variable and the outcome (23). More importantly, the association of chili pepper with mortality risk was independent of the adherence to the MD, supporting the notion that minor dietary changes, such as adding chilies to usual diet, could be valuable measures for improving health, especially cardiovascular health, independent of overall diet quality.

We also aimed to examine whether some biological mechanisms could be on the pathway between chili pepper and mortality. Our results showed that the majority of established biomarkers of CVD risk poorly accounted for the observed lower risk of death; only serum vitamin D was likely to explain a modest 


\begin{tabular}{|c|c|c|c|c|}
\hline & \multicolumn{4}{|c|}{ Chili Pepper Consumption } \\
\hline & $\begin{array}{l}\text { None/Rare Consumers } \\
\quad(n=7,689)\end{array}$ & $\begin{array}{l}\text { Consumers } \\
(n=15,122)\end{array}$ & PTE (\%) & PTE $p$ Value \\
\hline Cancer mortality, deaths & $173(2.2)$ & $309(2.0)$ & - & \\
\hline Model 2 & ref & $0.90(0.74-1.10)$ & - & \\
\hline Model $2+$ cardiac troponin & ref & $0.90(0.74-1.10)$ & Null & \\
\hline Model 2 + natriuretic peptide & ref & $0.91(0.74-1.10)$ & $1.2[0.0-71.4]$ & 0.35 \\
\hline Model 2 + biomarkers of renal function & ref & $0.90(0.74-1.10)$ & Null & \\
\hline Model 2 biomarkers of glucose metabolism & ref & $0.90(0.74-1.10)$ & Null & \\
\hline Model 2 + biomarkers of lipid metabolism & ref & $0.91(0.75-1.11)$ & $5.8[0.2-61.9]$ & 0.21 \\
\hline Model 2 + serum vitamin $\mathrm{D}(\mathrm{ng} / \mathrm{ml})$ & ref & $0.91(0.75-1.11)$ & $8.9[0.7-57.5]$ & 0.064 \\
\hline Model $2+$ inflammatory biomarkers & ref & $0.90(0.73-1.09)$ & Null & \\
\hline Model $2+$ BP + BMI & ref & $0.91(0.74-1.11)$ & $4.2[0.1-61.6]$ & 0.24 \\
\hline Other cause mortality, deaths & $173(2.2)$ & $309(2.0)$ & - & \\
\hline Model 2 & ref & $0.77(0.61-0.96)$ & - & \\
\hline Model $2+$ cardiac troponin & ref & $0.75(0.60-0.95)$ & Null & \\
\hline Model $2+$ natriuretic peptide & ref & $0.77(0.61-0.97)$ & Null & \\
\hline Model $2+$ biomarkers of renal function & ref & $0.77(0.61-0.97)$ & Null & \\
\hline Model 2 + biomarkers of glucose metabolism & ref & $0.77(0.61-0.96)$ & Null & \\
\hline Model 2 + biomarkers of lipid metabolism & ref & $0.78(0.62-0.99)$ & $7.7[1.2-35.6]$ & 0.10 \\
\hline Model 2 + serum vitamin $D(\mathrm{ng} / \mathrm{ml})$ & ref & $0.77(0.61-0.98)$ & $3.9[0.8-16.5]$ & 0.059 \\
\hline Model $2+$ inflammatory biomarkers & ref & $0.77(0.61-0.97)$ & Null & \\
\hline Model $2+$ BP + BMI & ref & $0.77(0.61-0.98)$ & $4.0[0.6-23.9]$ & 0.13 \\
\hline \multicolumn{5}{|c|}{ 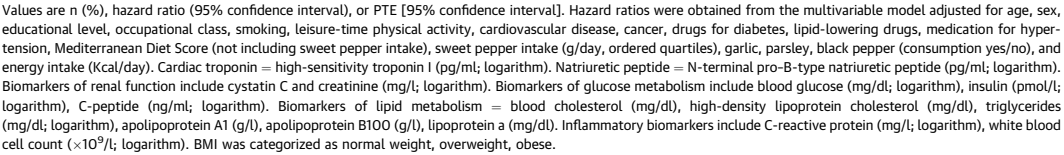 } \\
\hline \multicolumn{5}{|c|}{$\mathrm{BP}=$ blood pressure; $\mathrm{BMI}=$ body mass index; Null = not mediating the effect; $\mathrm{PTE}=$ percent of exposure effect; ref $=$ reference. } \\
\hline
\end{tabular}

proportion of the relation of chili pepper with total, CVD, and other-cause mortality death risk, while biomarkers of lipid metabolism appeared to slightly mediate the association of chilies with all-cause mortality.

In our population sample, regular chili pepper intake was associated with increased levels of lipids, a finding in agreement with previous epidemiological observations on serum triglycerides from a cohort of Chinese adults, but in apparent contrast with their reported inverse association of spicy food consumption with serum cholesterol (24). By contrast, a human intervention on 27 participants reported that regular consumption of freshly chopped chilies for 4 weeks had no effect on serum lipids and lipoproteins (25).

Other biological mechanisms potentially linking chili peppers to lower mortality risk, especially CVDrelated, could be ascribed to the weight losspromoting properties of chilies $(8,9,12)$ : protection against obesity leads indeed to decreased risk of cardiovascular and metabolic diseases; yet, in our population, regular chili pepper consumers were more likely to be obese than nonconsumers, and the inclusion of BMI in the mediating pathways did not substantially affect mortality risk.

Finally, in light of the well-known anti-inflammatory properties of chili peppers (26), we tested the role of inflammatory processes, by 2 widely used biomarkers but failed to detect any attenuation of risk; however, the use of only 2 markers could lead to an underestimation of the role of inflammation as a likely mediating mechanism linking chili pepper consumption to death risk.

None of the biological mechanisms tested were able to explain the health benefits associated with chilies; however, we could not explore other pathways that could link chili pepper intake to improved health outcomes, such as reduced oxidation or antiatherogenic potential (4).

Of note, an inverse association with total and IHD mortality was also observed for sweet peppers, which contain capsaicin in smaller, yet possibly beneficial, amounts, although no effect was observed toward CVD and cerebrovascular mortality risk; these findings suggest that the health advantages associated with chili peppers are likely to be ascribed to the high 


\begin{tabular}{|c|c|c|c|c|c|c|}
\hline & \multicolumn{3}{|c|}{ Total Mortality } & \multicolumn{3}{|c|}{ CVD Mortality } \\
\hline & Deaths/Subjects & HR $(95 \% \mathrm{Cl})$ & p Value for Interaction & Deaths & HR $(95 \% \mathrm{Cl})$ & p Value for Interaction \\
\hline Age $<65$ yrs & $337 / 17,613$ & $0.74(0.58-0.94)$ & 0.51 & 79 & $0.79(0.47-1.31)$ & 0.53 \\
\hline Age $\geq 65$ yrs & $899 / 5,198$ & $0.85(0.74-0.97)$ & & 365 & $0.78(0.63-0.97)$ & \\
\hline Women & $429 / 11,938$ & $0.84(0.69-1.02)$ & 0.79 & 162 & $0.93(0.67-1.28)$ & 0.29 \\
\hline Men & $807 / 10,873$ & $0.78(0.67-0.91)$ & & 282 & $0.66(0.51-0.84)$ & \\
\hline Up to lower secondary education & $896 / 11,918$ & $0.85(0.74-0.98)$ & 0.22 & 337 & $0.80(0.64-0.996)$ & 0.84 \\
\hline Upper secondary/post-secondary & $340 / 10,893$ & $0.73(0.57-0.92)$ & & 107 & $0.67(0.44-1.03)$ & \\
\hline Nonsmokers & $989 / 17,568$ & $0.84(0.74-0.96)$ & 0.71 & 367 & $0.85(0.69-1.06)$ & 0.20 \\
\hline Smokers & $247 / 5,243$ & $0.76(0.58-1.01)$ & & 77 & $0.53(0.33-0.85)$ & \\
\hline Low physical activity & $678 / 11,545$ & $0.82(0.70-0.96)$ & 0.79 & 256 & $0.85(0.66-1.11)$ & 0.25 \\
\hline High physical activity & $558 / 11,266$ & $0.79(0.66-0.95)$ & & 188 & $0.63(0.46-0.85)$ & \\
\hline Free from CVD & $956 / 21,274$ & $0.80(0.70-0.92)$ & 0.93 & 289 & $0.78(0.61-0.998)$ & 0.46 \\
\hline CVD subjects & $235 / 1,177$ & $0.82(0.62-1.08)$ & & 131 & $0.64(0.45-0.93)$ & \\
\hline Free from cancer & $1,105 / 21,985$ & $0.81(0.71-0.92)$ & 0.36 & 415 & $0.72(0.59-0.88)$ & 0.069 \\
\hline Cancer subjects & $116 / 736$ & $0.79(0.53-1.18)$ & & 25 & $1.72(0.61-4.85)$ & \\
\hline Free from diabetes & $1,016 / 21,442$ & $0.82(0.72-0.94)$ & 0.75 & 1,016 & $0.74(0.59-0.92)$ & 0.70 \\
\hline Subjects with diabetes & $188 / 1,088$ & $0.70(0.51-0.96)$ & & 80 & $0.80(0.48-1.31)$ & \\
\hline Free from hyperlipidemia & $1,045 / 20,849$ & $0.82(0.72-0.94)$ & 0.88 & 359 & $0.79(0.63-0.98)$ & 0.86 \\
\hline Subjects with hyperlipidemia & $169 / 1,756$ & $0.83(0.59-1.16)$ & & 76 & $0.77(0.46-1.29)$ & \\
\hline Free from hypertension & $563 / 16,203$ & $0.68(0.57-0.81)$ & 0.021 & 141 & $0.67(0.47-0.94)$ & 0.54 \\
\hline Subjects with hypertension & $637 / 6,231$ & $0.94(0.80-1.12)$ & & 287 & $0.82(0.64-1.05)$ & \\
\hline Low MD (MDS 0-3) & $384 / 6,898$ & $0.91(0.73-1.12)$ & 0.32 & 142 & $0.85(0.60-1.20)$ & 0.62 \\
\hline Average MD (MDS 4-5) & $545 / 10,053$ & $0.73(0.61-0.88)$ & & 196 & $0.77(0.57-1.04)$ & \\
\hline High MD (MDS 6-9) & $307 / 5,860$ & $0.85(0.66-1.10)$ & & 106 & $0.64(0.42-0.97)$ & \\
\hline Healthy subjects* & $710 / 19,479$ & $0.84(0.71-0.98)$ & - & 224 & $0.74(0.56-0.97)$ & - \\
\hline Excluding early deaths (follow-up $>2$ yrs) & $1,067 / 22,642$ & $0.81(0.71-0.92)$ & - & 373 & $0.78(0.63-0.96)$ & - \\
\hline
\end{tabular}

content of capsaicin, which is far more abundant in chili than in sweet nonspicy peppers. Similar conclusions were reached by a study revealing a positive correlation between frequency of chili consumption, but not sweet peppers, and muscle strength in adult males (27). However, Capsicum species contain a large variety of phytochemicals with well-known antioxidant properties, such as carotenoids ( $\beta$-carotene), capsaicinoids (capsaicin), and flavonoids (quercetin and luteolin) $(28,29)$. Therefore, a possible synergistic activity of these bioactive compounds cannot be ruled out.

STUDY STRENGTHS AND LIMITATIONS. Major strengths of this study include a large sample size, a prospective cohort design, and careful ascertainment of established and potential risk factors for death; moreover, analyses were controlled for several dietary covariates that may be correlated with intake of chili peppers. Finally, it is the first investigation addressing the association of chili pepper intake and mortality risk in a large Mediterranean population.

The present study has, however, several limitations. First, given the observational nature of our investigation, causality can only be suggested, and residual confounding or confounding by unmeasured factors cannot be fully ruled out.

Second, cause-specific mortality analysis in this dataset are limited by the relatively small number of deaths. Lastly, subjects' information was collected at baseline only, thus changes that may have occurred during the follow-up could not be considered.

\section{CONCLUSIONS}

Regular consumption of chili peppers is associated with lower risk of total and CVD mortality in a large Mediterranean cohort of adults. The mechanisms through which chili peppers could lower mortality risk are still unclear, although a modest role was found for traditional CVD risk factors. The inclusion of chili pepper intake into a traditional MDS offered no or small added value in the discrimination ability of the modified score. To the best of our knowledge, this study is the first to report a negative association between chili pepper intake and (all-cause and specific) mortality risk in a Mediterranean prospective 
cohort, and to assess possible biological mechanisms underlying such association.

ACKNOWLEDGMENTS The Moli-sani Study research group thanks the Associazione Cuore Sano ONLUS (Campobasso, Italy) for its support and is grateful to the BiomarCaRE Consortium.

ADDRESS FOR CORRESPONDENCE: Dr. Marialaura Bonaccio, Department of Epidemiology and Prevention, IRCCS Neuromed, Via dell'Elettronica, 86077 Pozzilli (IS), Italy. E-mail: marialaura.bonaccio@ neuromed.it. Twitter: @Mbonaccio.

\section{PERSPECTIVES}

COMPETENCY IN MEDICAL KNOWLEDGE: In an Italian population, regular consumption of chili peppers is associated with a lower long-term risk of mortality from cardiovascular causes.

TRANSLATIONAL OUTLOOK: Further studies are needed to understand the biological mechanisms responsible for apparent beneficial effect of chili peppers on cardiovascular risk.

\section{REFERENCES}

1. Gerber M, Hoffman R. The Mediterranean diet: health, science and society. $\mathrm{Br}$ J Nutr 2015;113 Suppl 2:54-10.

2. Martínez-González MÁ, Hershey MS, Zazpe Trichopoulou A. Transferability of the Mediterranean diet to non-Mediterranean countries. What is and what is not the Mediterranean diet. Nutrients 2017;9:E1226.

3. Shen J, Wilmot KA, Ghasemzadeh $N$, et at. Mediterranean dietary patterns and cardiovascular health. Annu Rev Nutr 2015;35:425-49.

4. Tsui PF, Lin CS, Ho LJ, Lai JH. Spices and atherosclerosis. Nutrients 2018:10:E1724.

5. Sun F, Xiong S, Zhu Z. Dietary capsaicin protects cardiometabolic organs from dysfunction. Nutrients 2016;8:E174.

6. Fernandes ES, Cerqueira AR, Soares AG, Costa SK. Capsaicin and its role in chronic diseases. Adv Exp Med Biol 2016;929:91-125.

7. López-Carrillo L, Hernández Avila M, Dubrow R. Chili pepper consumption and gastric cancer in Mexico: a case-control study. Am J Epidemio 1994;139:263-71.

8. Varghese $S$, Kubatka $P$, Rodrigo L, et al. Chil pepper as a body weight-loss food. Int J Food Sc Nutr 2017;68:392-401.

9. Shi Z, Riley M, Taylor AW, Page A. Chilli consumption and the incidence of overweight and obesity in a Chinese adult population. Int J Obes 2017;41:1074-9.

10. Kang C, Wang B, Kaliannan $K_{\text {, et al. Gut }}$ microbiota mediates the protective effects of $d$ etary capsaicin against chronic low-grade inflammation and associated obesity induced by high-fat diet. MBio 2017;8. e00470-17.

11. Lv J, Qi L, Yu C, et al. China Kadoorie Biobank Collaborative Group. Consumption of spicy foods and total and cause specific mortality: population based cohort study. BMJ 2015;351:h3942.

12. Chopan $M_{r}$ Littenberg $B$. The association of ho red chili pepper consumption and mortality: large population-based cohort study. PLoS One 2017;12:e0169876.

13. Di Castelnuovo $A$, Costanzo $S$, Persichillo $M$ et al. Distribution of short and lifetime risks for cardiovascular disease in Italians. Eur J Prev Cardiol 2012;19:723-30.

14. Bonaccio $M$, Di Castelnuovo $A$, Pounis $G$, et al, Moli-sani Study Investigators. Relative contribution of health-related behaviours and chronic diseases to the socioeconomic patterning of lowgrade inflammation. Int J Public Health 2017;62 $551-62$.

15. Pisani P, Faggiano F, Krogh V, et al. Relative validity and reproducibility of a food frequency dietary questionnaire for use in the Italian EPIC centres. Int J Epidemiol 1997;26 Suppl 1:S152-60.

16. Trichopoulou A, Costacou T, Bamia $C_{1}$ Trichopoulos D. Adherence to a Mediterranean diet and survival in a Greek population. N Engl Med 2003;348:2599-608.

17. Pounis G, Costanzo S, di Giuseppe $R$, et at. Consumption of healthy foods at different conter of antioxidant vitamins and phytochemicals and metabolic risk factors for cardiovascular disease in men and women of the Moli-sani study. Eur J Clin Nutr 2013:67:207-13.

18. Pounis $G$, Bonaccio $M$, Di Castelnuovo $A$, et al. Polyphenol intake is associated with low-grad inflammation, using a novel data analysis from the Moli-sani study. Thromb Haemost 2016;115: 344-52.

19. Jeurnink $S M$, Büchner $F L$, Bueno-deMesquita $H B$, et al. Variety in vegetable and fruit consumption and the risk of gastric and esophageal cancer in the European Prospective Investigation into Cancer and Nutrition. Int J Cancer 2012;131:E963-73.

20. Zeller $T$, Hughes $M$, Tuovinen $T$, et al. BiomarCaRE: rationale and design of the Europea BiomarCaRE project including 300,000 participants from 13 European countries. Eur J Epidemio 2014;29:777-90.
21. Hertzmark E, Pazaris M, Spiegelman D. The SAS MEDIATE Macro. Boston, MA: Harvard T.H. Chan School of Public Health, 2012.

22. Pencina MJ, D'Agostino RB Sr., Steyerberg EW. Extensions of net reclassification improvement calculations to measure usefulness of new bio markers. Stat Med 2011;30:11-21.

23. Lo A, Chernoff H, Zheng T, Lo SH. Why significant variables aren't automatically good predictors. Proc Natl Acad Sci U S A 2015:112:13892-7.

24. Xue $Y, H e T, Y u K$, et al. Association between spicy food consumption and lipid profiles in adults: a nationwide population-based study. $\mathrm{Br}$ J Nutr 2017;118:144-53.

25. Ahuja $K D$, Ball MJ. Effects of daily ingestion of chilli on serum lipoprotein oxidation in adult men and women. Br J Nutr 2006;96:239-42.

26. Luo XJ, Peng J, Li Y YJ. Recent advances in the study on capsaicinoids and capsinoids. Eur Pharmacol 2011;650:1-7.

27. Wu H, Wei M, Zhang $Q$, et al. Consumption of chilies, but not sweet peppers, is positively relate to handgrip strength in an adult population. J Nutr Health Aging 2016;20:546-52.

28. Materska M, Perucka I. Antioxidant activity of the main phenolic compounds isolated from hot pepper fruit (Capsicum annuum L). J Agric Food Chem 2005:53:1750-6.

29. Hervert-Hernández D, Sáyago-Ayerdi SG, Goñi I. Bioactive compounds of four hot pepper varieties (Capsicum annuum L.), antioxidant capacity, and intestinal bioaccessibility. J Agric Food Chem 2010:58:3399-406.

KEY WORDS cardiovascular mortality, cerebrovascular mortality, chili pepper, inflammation, Mediterranean diet, risk factors, total mortality

APPENDIX For supplemental tables and list of the Moli-sani Study Investigators, please see the online version of this paper. 



\section{CHAPTER 8.}

Daily Coffee Drinking Is Associated with Lower Risks of Cardiovascular and Total Mortality in a General Italian Population: Results from the Moli-sani Study

Ruggiero E, Di Castelnuovo A, Costanzo S, Persichillo M, De Curtis A, Cerletti C, Donati MB, de Gaetano G, Iacoviello L, Bonaccio M, on behalf of the Moli-sani Study Investigators.

J Nutr. 2020 Dec 31; nхaа365. 



\title{
Daily Coffee Drinking Is Associated with Lower Risks of Cardiovascular and Total Mortality in a General Italian Population: Results from the Moli-sani Study
}

\author{
Emilia Ruggiero, ${ }^{1}$ Augusto Di Castelnuovo, ${ }^{2}$ Simona Costanzo, ${ }^{1}$ Mariarosaria Persichillo, ${ }^{1}$ Amalia De \\ Curtis, ${ }^{1}$ Chiara Cerletti, ${ }^{1}$ Maria Benedetta Donati, ${ }^{1}$ Giovanni de Gaetano, ${ }^{1}$ Licia Iacoviello,,${ }^{1,3}$ and \\ Marialaura Bonaccio ${ }^{1}$ for the Moli-sani Study Investigators \\ ${ }^{1}$ Department of Epidemiology and Prevention, IRCCS NEUROMED, Pozzilli, Italy; ${ }^{2}$ Mediterranea Cardiocentro, Napoli, Italy; and \\ ${ }^{3}$ Department of Medicine and Surgery, Research Center in Epidemiology and Preventive Medicine, University of Insubria, Varese-Como, \\ Italy
}

\begin{abstract}
Background: An inverse relationship between coffee intake and mortality has been observed in several population cohorts, but rarely within Mediterranean countries. Moreover, the biological pathways mediating such an association remain unclear.

Objectives: We assessed the associations between coffee consumption and total and cause-specific mortality and examined the mediating roles of N-terminal pro B-type natriuretic peptide (NTproBNP), high-sensitivity Troponin I, blood glucose, lipid metabolism, and selected biomarkers of inflammation and renal function.

Methods: We longitudinally analyzed data on 20,487 men and women (35-94 years old at baseline) in the Moli-sani Study, a prospective cohort established in 2005-2010. Individuals were free from cardiovascular disease (CVD) and cancer and were followed-up for a median of 8.3 years. Dietary data were collected by a 188 -item semi-quantitative FFQ. Coffee intake was standardized to a $30-\mathrm{mL}$ Italian espresso cup size. HRs with $95 \% \mathrm{Cls}$ were calculated by multivariable Cox regression.

Results: In comparison with no/rare coffee consumption (up to $1 \mathrm{cup} / \mathrm{d}$ ), HRs for all-cause mortality across categories of coffee consumption ( $>1$ to $\leq 2,>2$ to $\leq 3,>3$ to $\leq 4$ and $>4$ cups/d) were $0.79(95 \% \mathrm{Cl}, 0.65-0.95), 0.84(95 \% \mathrm{Cl}$, $0.69-1.03), 0.72(95 \% \mathrm{Cl}, 0.57-0.92)$, and $0.85(95 \% \mathrm{Cl}, 0.62-1.12)$, respectively. For CVD mortality, a nonlinear $(P$ for non-linearity $=0.021$ ) $\mathrm{J}$-shaped association was found (magnitude of the relative reduction $=37 \%$; nadir at 3-4 cups/d). Circulating levels of NTproBNP explained up to $26.4 \%$ of the association between coffee and all-cause mortality, while systolic blood pressure was likely to be on the pathway between coffee and CVD mortality, although to a lesser extent. Conclusions: In this large cohort of Italian adults, moderate consumption (3-4 cups/d) of Italian-style coffee was associated with lower risks of all-cause and, specifically, of CVD mortality. Among the known biomarkers investigated here, NTproBNP likely mediates the relationship between coffee intake and all-cause mortality. J Nutr 2020;0:1-10.
\end{abstract}

Keywords: coffee consumption, mortality risk, cardiovascular mortality, general population, Mediterranean diet

\section{Introduction}

Coffee is among the most commonly consumed nonalcoholic beverages worldwide and contains various antioxidants and phenolic compounds, such as flavonoids and caffeine, some of which have been shown to exert beneficial effects for health (1-3).

The relationships between coffee intake and the risks of disease/mortality have been widely investigated in a number of epidemiological settings, most of which indicate a favorable role of regular coffee intake on a variety of health outcomes, including cardiovascular disease (CVD), mortality, and cancer $(4,5)$. Regarding cardiovascular health, the currently available evidence on CVD effects related to habitual coffee consumption is largely reassuring, suggesting that coffee can be included as part of a healthy diet for the general public and also for those with an increased cardiovascular risk or CVD (6).

More recently, a meta-analysis of 40 prospective cohort studies found lower risks of mortality from all causes and from CVD in a comparison of the highest versus lowest coffee consumption categories, with similar results across various subpopulations by characteristics of subjects, including age, 
sex, overweight status, alcohol drinking, smoking status, and caffeine content consumed (7).

Although the mechanisms of action are not entirely understood, the health-promoting properties of coffee are largely attributed to its rich phytochemistry, including caffeine, chlorogenic acid, caffeic acid, and hydroxy hydroquinone (8), and to its antioxidant compounds, which may explain the potential benefits of coffee against those conditions associated with a chronic state of subclinical inflammation, such as CVD and cancer $(9,10)$.

Though largely consumed among Mediterranean populations, coffee has generally not been included among the food items used to obtain Mediterranean dietary scores (11), nor has coffee consumption been extensively investigated among Mediterranean countries; the relative paucity of studies renders it difficult to provide definitive evidence on whether an antioxidant-rich beverage may have potential health benefits among populations already consuming a high-antioxidant diet, such as the Mediterranean diet (MD).

The primary aim of this study was to prospectively assess the association of coffee consumption with total and cause-specific mortality in a large Italian population of adult men and women; as a secondary aim, we examined several biological mechanisms that might explain the relationship between coffee intake and mortality by analyzing the possible contributions of selected biomarkers of different biological processes that predispose individuals to CVD onset or progression. Finally, we assessed whether the inclusion of coffee consumption would improve risk predictions associated with a traditional Mediterranean Diet Score (MDS).

The enrollment phase of the Moli-sani Study was supported by unrestricted research grants from the Pfizer Foundation (Rome, Italy), the Italian Ministry of University and Research (MIUR, Rome, Italy)-Programma Triennale di Ricerca, Decreto number 1588, and Instrumentation Laboratory, Milan, Italy. The follow-up phase of the Moli-sani Study (assessment of incident cases) was partially supported by the Italian Association for Cancer Research (AIRC) "5xMILLE" (HYPERCAN Study, number 12237) and the Italian Ministry of Health (Principal Investigator, GdG; Co-Principal Investigator, SC; grant number RF-2018-12367074). The present analyses were partially supported by a grant to MB from the Italian Ministry of Health 2013 (grant number GR-2013-02356060), by the Hypercan Study, AIRC "5xMILLE" number 12237, and by POR FESR (Programmi Operativi Regionali -Fondo Europeo di Sviluppo Regionale) 20142020: DD number 459 of 27/11/2018, SATIN (Sviluppo di Approcci Terapeutici INnovativi per patologie neoplastiche resistenti ai trattamenti) and by a grant to $\mathrm{LI}$ as a partner of BiomarCaRE (Biomarkers for Cardiovascular Risk Assessment in Europe) by European Commission Seventh Framework Programme FP7/20072013 (HEALTH-F2-2011-278913).

The funders had no role in the study design, collection, analysis, and interpretation of data; in writing the manuscript; or in the decision to submit the article for publication. All authors were and are independent from funders. Author disclosures: MB was supported by a Fondazione Umberto Veronesi Fellowship. SC was the recipient of a Fondazione Umberto Veronesi travel grant. All the other authors report no conflicts of interest.

Supplemental Tables 1-7 and Supplemental Figure 1 are available from the "Supplementary data" link in the online posting of the article and from the same link in the online table of contents at https://academic.oup.com/jn/. Address correspondence to LI (e-mail: licia.iacoviello@neuromed.it ). Abbreviations used: CRP C-reactive protein: CVD cardiovascular disease: EPIC, European Prospective Investigation into Cancer and Nutrition; ICD9. International Classification of Diseases, Ninth Edition; IDI, integrated discrimination improvement; IHD, ischemic heart disease; MD, Mediterranean diet; MDS, Mediterranean diet score; NRI, Net reclassification index; NTproBNP, $\mathrm{N}$-terminal pro B-type natriuretic peptide; PA, physical activity; WBC, white blood cell count.

\section{Methods}

\section{Study population}

The Moli-sani Study is a large, population-based cohort study that, in 2005-2010, randomly recruited 24,325 men and women aged $\geq 35$ years from the adult general population of the Molise region, a central-southern area of Italy, from city hall registries by multistage sampling. The exclusion criteria were pregnancy, disturbances in mental or decision-making impairments, ongoing poly traumas, or coma.

Details of the study are provided elsewhere (12). For the purpose of the present analyses, we excluded subjects with a history of CVD ( $n=1188)$ or missing data on CVD $(n=366)$, with a history of cancer $(n=697)$ or missing data on cancer $(n=87)$, reporting implausible energy intakes $(<800 \mathrm{kcal} / \mathrm{d}$ in men and $<500 \mathrm{kcal} / \mathrm{d}$ in women or $>4000 \mathrm{kcal} / \mathrm{d}$ in men and $>3500 \mathrm{kcal} / \mathrm{d}$ in women; $n=371$ ), with unreliable dietary or medical questionnaires $(n=955$ and $n=60$, respectively), lost to follow-up $(n=22)$, without information on causespecific death $(n=15)$, or with missing information for covariates ( $n=77$ ). Those participants with missing values of 1 or more covariates (missing data: diabetes, $n=281$; hypertension, $n=378$; and hyperlipidaemia, $n=206$ ) were kept in the data set and treated as missing reporting, but were excluded in sensitivity analysis. The final sample was of 20,487 individuals (Supplemental Figure 1).

The cohort was followed-up for mortality until 31 December 2015. Overall and cause-specific mortality rates were assessed by the Italian mortality registry (Registro Nominativo Cause Morte, ReNCaM registry), validated by Italian death certificates (Istituto Nazionale di Statistica, ISTAT), and coded according to the International Classification of Diseases, Ninth Edition (ICD-9).

CVD mortality included deaths from diseases of the circulatory system when the underlying cause of death included ICD-9 codes 390459. ICD-9 codes 430-438 were used to define a specific cause of death for cerebrovascular disease and ICD-9 codes 410-414 and 429 were used to define a specific cause of death for ischemic heart disease (IHD). Cancer was considered as the cause of death when using ICD-9 codes 140-208. Noncardiovascular/noncancer causes of death were included in an "other-cause mortality" group.

The Moli-sani Study complies with the Declaration of Helsinki and was approved by the Ethical Committee of the Catholic University Medical School in Rome, Italy. All participants provided written informed consent.

\section{Dietary assessment}

Dietary intake was assessed by an interviewer-administered, semiquantitative, European Prospective Investigation into Cancer and Nutrition (EPIC) FFQ that was validated and adapted to the Italian population to assess participants' diet during the past 12 months.

The FFQ includes 188 food items, classified into 74 predefined food groups on the basis of similar nutrient characteristics or culinary usage (13). Participants were asked to indicate the number of times a given item was consumed (per day, week, month, or year), from which the frequency of consumption was calculated. The quantity of food consumed was assessed by asking the participant to select 1 among several images of different food portions or by using a predefined standard portion when no image was available.

Frequencies and quantities of each food were then linked, using specifically designed software (14), to Italian Food Tables (15) to obtain estimates of daily intakes of macro- and micro-nutrients plus energy.

For coffee, the questionnaire inquired about the consumption frequency of caffeinated and decaffeinated coffee, the 2 recipes mainly consumed in Italy, in the form of espresso or percolated (mocha) coffee. The question asked for coffee was "How many cups of coffee do you usually drink?," with possible answers being number of cups per day, week, or month, or never/almost never. Subsequently, participants were asked to indicate the type of coffee more usually consumed: that is, Italian espresso $(30 \mathrm{~mL})$, mocha coffee $(50 \mathrm{~mL})$, or decaffeinated coffee (30 mL). Cappuccino and café latte were assumed to contain $20 \%$ coffee and $80 \%$ milk. We calculated total coffee consumption as the sum of all these types of coffee. 
For the data analysis, we standardized the amount of coffee consumed to a $30-\mathrm{mL}$ cup size, which is the standard cup size of an Italian espresso according to the Italian dietary guidelines (16).

Coffee consumption was categorized as up to $1 \mathrm{cup} / \mathrm{d},>1$ to $\leq 2$ cups/d, $>2$ to $\leq 3 \mathrm{cups} / \mathrm{d},>3$ to $\leq 4 \mathrm{cups} / \mathrm{d}$ and $>4 \mathrm{cups} / \mathrm{d}$.

Added sugar was the quantity of sugar $(\mathrm{g} / \mathrm{d})$ added to beverages (i.e., milk, coffee, tea, or yogurt).

Adherence to the traditional MD was evaluated by the MDS developed by Trichopoulou et al. (11). The score was obtained by assigning 1 point each to foods considered healthy (fruits and nuts, vegetables, legumes, fish, cereals; MUFA to SFA ratio) whose consumption was above the sex-specific medians of intake of the Molisani study population for participants apparently free from CVD, cancer, and diabetes, and then the score was applied to the whole population; foods presumed to be detrimental (meat and dairy products were scored positively if their consumption was below the median. Al other intakes received 0 points. For ethanol, men who consumed 10 $50 \mathrm{~g} / \mathrm{d}$ and women who consumed $5-25 \mathrm{~g} / \mathrm{d}$ received 1 point; otherwise, the score was 0 . The MDS ranged from 0 to 9 (the latter reflecting maximal adherence) and scores were categorized according to poor ( 0 $3)$, average (4-5), and good adherence (6-9) to the MD.

\section{Assessment of covariates}

At baseline, information on socio-demographic variables, lifestyles, and medical history were obtained by interviewer-administered questionnaires.

The education variable was based on the highest qualification attained and was categorized as up to lower secondary (approximately $\leq 8$ years of study), upper secondary education (8-13 years of study), and post-secondary or higher ( $>13$ years of study). Housing status was classified as rented, 1-dwelling ownership, or $>1$-dwelling ownership.

Subjects were classified as never, current, or former smokers (quit at least 1 year ago) and, among ever smokers, the number of cigarette smoked per day was also assessed. Leisure-time physical activity (PA) for sports, walking, and gardening was dichotomized as $<30$ or $\geq 30 \mathrm{~min} / \mathrm{d}$.

Waist circumference (in $\mathrm{cm}$ ) was measured between the twelfth rib and the iliac crest, and hip circumference (in $\mathrm{cm}$ ) was measured around the buttocks. The waist-to-hip ratio was then calculated, and abdominal obesity was defined as a waist-to-hip ratio $\geq 0.90$ for men and $\geq 0.85$ for women (17).

Participants were considered to have diabetes, hypertension, or hyperlipidemia at baseline if they were taking disease-specific drugs.

\section{Selection and assessment of biomarkers of CVD risk}

The biomarkers tested in the present study were selected on the basi whether they were: 1) previously studied for their relevance in pathways predisposed to CVD; 2 ) shown in epidemiologic studies to be related to CVD or mortality; and 3) already introduced in the analyses of the Moli-sani Study cohort.

Each of these biomarkers reportedly reflects different underlying pathways to disease incidence and progression $(18,19)$.

Blood samples were collected at baseline in participants who had fasted overnight and had refrained from smoking for at least 6 hours lipids (total cholesterol, HDL, triglycerides) and blood glucose were assayed in serum samples by enzymatic reaction methods using an automatic analyzer (ILab 350, Instrumentation Laboratory, Milan, Italy); quality control for lipids and glucose was obtained by a commercial standard (SeraChem 1 and SeraChem2). For SeraChem1 and SeraChem2, the coefficients of variability were $4.9 \%$ and $5.2 \%$ respectively for blood cholesterol; $3.2 \%$ and $3 \%$ for HDL $5.2 \%$ and $5.3 \%$ for triglycerides; and $4.7 \%$ and $4.1 \%$ for blood glucose.

High sensitivity C-reactive protein (CRP) was measured in fresh serum samples by a particle-enhanced immune-turbidimetric assay (ILab 350, Instrumentation Laboratory, Milan, Italy). Quality control for CRP was maintained using an in-house serum pool and a commer cial laboratory standard whose inter-day coefficients of variability were $5.5 \%$ and $4.2 \%$, respectively.
A hemocromocytometric analysis was performed by cell count (Coulter HMX, Beckman Coulter, IL, Milan, Italy) within 3 hours of blood collection. Quality control was performed using 3 different levels of standards: Abnormal 1, a pathologically high control; Abnormal 2, a pathologically low control; and Normal (Coulter HMX, Beckman Coulter). Coefficients of variability for white blood cells (WBC) were $6.2 \%, 3.3 \%$, and $3.0 \%$ for Abnormal 1, Abnormal 2, and Normal, respectively. N-terminal pro B-type natriuretic peptide (NTproBNP), high-sensitivity assayed Troponin I, apoA, apoB100, lipoprotein a, markers of renal function (cystatin C, creatinine), insulin, C-peptide, and serum vitamin D were measured subsequently on thawed samples stored frozen in liquid nitrogen at the biological bank of the Moli-sani study, in the framework of the collaborative BiomarCaRE (Biomarker for Cardiovascular Risk Assessment in Europe) research project, whose primary objective is to assess the value of established and emerging biomarkers for CVD risk prediction by using data from 23 cohorts across Europe (18).

The low-grade inflammation (INFLA) score was constructed by summarizing synergistic effects of inflammatory biomarkers (20). Briefly, deciles of each biomarker level (CRP, WBC, platelets, and the granulocyte-to-lymphocyte ratio) were generated. For all 4 components, being in the highest deciles (7-10) gave a score that increased from 1 to 4 , while being in the lowest deciles (1-4) was negatively scored from -4 to -1 . Being in deciles 5 or 6 was scored as 0 points. In such a way, the INFLA score ranged from between -16 and 16 and came up as the sum of the 4 biomarkers. An increase in the score represented an increase in low-grade inflammation intensity.

\section{Statistical analysis}

Baseline characteristics of the participants by categories of daily coffee intake were presented as means and SDs or as percentages for categorical variables. Differences in the distribution of baseline covariates were calculated using an ANOVA adjusted for age, sex, and energy intake (GENMOD procedure for categorical variables and general linear model procedure for continuous variables in SAS software; Table 1). Positively skewed variables were log transformed before analysis.

Risk estimates for all-cause and cause-specific deaths were expressed as HRs with $95 \%$ CIs and were calculated using Cox regression models with time-on-study on the time scale and adjusting for baseline age as a covariate in the model.

On the basis of previous literature and biological plausibility, 2 models for the association of coffee with the mortality risk were fitted: Model 1 was adjusted for age (continuous), sex, and energy intake (kcal; continuous); Model 2 was adjusted as in Model 1 and further controlled for education (up to lower secondary school; upper secondary school; postsecondary/higher), housing (rented and 1- or $>1$ dwelling ownership), residence (urban/rural), leisure-time PA $(<30$ or $\geq 30 \mathrm{~min} / \mathrm{d}$ ), smoking (never, current, former smokers), number of cigarettes per day $(\leq 5,>5$ to $\leq 10,>10$ to $\leq 20,>20$, and missing data), abdominal obesity (no/yes), diabetes (no/yes/missing), hyperlipidaemia (no/yes/missing), hypertension (no/yes/missing), MDS (continuous), tea intake (nonconsumers, $>0$ to $\leq 2 \mathrm{cups} /$ week, $>2$ to $\leq 4 \mathrm{cups} /$ week, and $>4$ cups/week), and added sugar (g/d; continuous). Missing values of hypertension, hyperlipidaemia, and diabetes were included in the model as dummy variables, similar to the way valid categories were represented.

For a biomarker to be considered as mediating the association of coffee intake and mortality, it had to be associated with both the exposure and the outcome (at least 1 of those analyzed) with a $P$ value $<0.20$, in accordance with predefined mediation principles $(21,22)$. These criteria were tested in distinct multivariable regression models for each potential mediator individually (Supplemental Table 1) and through Cox models that included coffee consumption as a covariate (Supplemental Table 2).

The multivariable Model 2 served as the reference for the mediation analysis used to estimate the contribution of each set of potential mediators, which were alternately included in Model 2.

We performed our analysis on 18,661 subjects (after exclusion of those individuals with missing data on any of the biomarkers) to 
TABLE 1 Selected characteristics of participants at baseline in the Moli-sani Study cohort $(n=20,487)$ across categories of daily coffee intake

\begin{tabular}{|c|c|c|c|c|c|c|}
\hline & \multicolumn{5}{|c|}{ Coffee intake, cups/d } & \multirow[b]{2}{*}{$P$ value } \\
\hline & $\leq 1$ & $>1$ to $\leq 2$ & $>2$ to $\leq 3$ & $>3$ to $\leq 4$ & $>4$ & \\
\hline Participants, $n(\%)$ & $3714(18.1)$ & $4436(21.7)$ & $4680(22.8)$ & $3747(18.3)$ & $3910(19.1)$ & \\
\hline Age, years & $58.1 \pm 12.8$ & $57.3 \pm 11.9$ & $55.7 \pm 11.6$ & $54.2 \pm 10.9$ & $51.3 \pm 9.3$ & $<0.0001$ \\
\hline Men & 47.8 & 47.2 & 46.5 & 44.1 & 49.3 & 0.0002 \\
\hline Educational level & - & - & - & - & - & $<0.0001$ \\
\hline Up to lower secondary & 58.9 & 54.7 & 51.5 & 45.7 & 44.2 & \\
\hline Upper secondary & 30.7 & 33.3 & 34.9 & 39.5 & 40.6 & \\
\hline Postsecondary & 10.4 & 12.0 & 13.6 & 14.8 & 15.2 & \\
\hline Housing & - & - & - & - & - & 0.13 \\
\hline Rent & 8.1 & 7.8 & 8.6 & 9.1 & 11.0 & \\
\hline 1 dwelling ownership & 84.0 & 82.9 & 82.8 & 81.8 & 80.2 & \\
\hline$>1$ dwelling ownership & 7.9 & 9.3 & 8.6 & 9.1 & 8.8 & \\
\hline Smoking status & - & - & - & - & - & $<0.0001$ \\
\hline Nonsmokers & 61.2 & 55.6 & 50.8 & 48.1 & 35.2 & \\
\hline Current smokers & 12.3 & 17.3 & 21.9 & 27.2 & 41.2 & \\
\hline Former smokers & 26.5 & 27.1 & 27.3 & 24.7 & 23.6 & \\
\hline Number of cigarettes smoked per day ${ }^{1}$ & - & - & - & - & - & $<0.0001$ \\
\hline$\leq 5$ & 33.6 & 30.5 & 22.0 & 21.1 & 15.1 & \\
\hline$>5$ to $\leq 10$ & 24.8 & 25.0 & 24.1 & 23.5 & 19.2 & \\
\hline$>10$ to $\leq 20$ & 31.4 & 36.7 & 43.4 & 44.9 & 48.1 & \\
\hline$>20$ & 9.4 & 7.0 & 9.7 & 9.6 & 17.1 & \\
\hline Physical activity $\geq 30 \mathrm{~min} / \mathrm{d}$ & 65.5 & 65.6 & 63.8 & 63.2 & 61.6 & 0.0002 \\
\hline Abdominal obesity & 75.1 & 73.6 & 72.8 & 72.2 & 70.1 & 0.0002 \\
\hline Diabetes & 3.8 & 4.8 & 3.8 & 4.0 & 3.4 & 0.0003 \\
\hline Hypertension & 30.9 & 29.4 & 24.7 & 20.7 & 15.2 & 0.0004 \\
\hline Hyperlipidemia & 6.1 & 6.0 & 5.4 & 5.5 & 3.5 & 0.0003 \\
\hline Mediterranean diet score & - & - & - & - & - & $<0.0001$ \\
\hline Poor: 0-3 & 25.9 & 29.4 & 30.5 & 32.7 & 35.0 & \\
\hline Average: 4-5 & 44.6 & 43.2 & 44.0 & 44.5 & 43.6 & \\
\hline Good: 6-9 & 29.5 & 27.4 & 25.5 & 22.8 & 21.4 & \\
\hline Added sugar, $\mathrm{g} / \mathrm{d}$ & $4.2(4.5)$ & $7.2(5.6)$ & $9.7(7.1)$ & $12.1(8.7)$ & $17.6(14.6)$ & $<0.0001$ \\
\hline Tea intake, cups/week & $1.4(3.5)$ & $0.9(2.5)$ & $0.7(2.1)$ & $0.7(2.2)$ & $0.6(2.2)$ & $<0.0001$ \\
\hline Alcohol consumption, g/d & $17.4(22.9)$ & $18.2(23.7)$ & $16.3(21.5)$ & $14.5(19.9)$ & $14.5(19.3)$ & $<0.0001$ \\
\hline
\end{tabular}

Values are expressed as percentages for all variables, except for age, added sugar, tea intake, and alcohol consumption, which are presented as means \pm SDs. Means and $P$ values are adjusted for sex, age, and energy intake. Coffee intake was standardized to a 30-mL Italian espresso cup size. Tea intake was standardized to a $150-\mathrm{mL}$ cup size.

${ }^{1}$ Percentages were calculated based on the total number of smokers $(n=4881)$.

evaluate how much of the associations between coffee consumption and all-cause or cause-specific mortality would be accounted for by biomarkers representative of different biological processes.

For the mediation analysis, the \% MEDIATE macro in SAS (23) was used to calculate the point and interval estimates of the percentage of exposure effect explained by 1 or more intermediate variables, with $95 \%$ CIs and $P$ values. Biomarkers were entered into the mediation analysis as continuous variables.

We conducted several analyses to assess the sensitivity of the observed associations to various baseline risk factors: age (35-64 and $\geq 65$ years), sex, socioeconomic strata (education and housing), lifestyles (smoking status, leisure-time PA, MDS), and health conditions at baseline (abdominal obesity, diabetes, hypertension, and hyperlipidaemia); we also conducted an analysis after excluding those subjects who died during the first 2 years of follow-up. Appropriate multiplicative terms for testing interactions were included in the multivariable models to test for a difference of effect of coffee intake across subgroups (Supplemental Table 3).

Possible curvilinear associations between the continuous measure of coffee intake (cups/d) and the all-cause and cause-specific mortality risks were tested via a multivariable Cox model using a restricted cubic spline with 3 knots at fixed percentiles (5th, 50th, and 95th percentiles of the distribution; Figure 1).
In order to see whether the inclusion of coffee would possibly improve risk predictions associated with MDS, we scored coffee consumption as previously done for the food groups already present in the MDS (11). For consistency with the methodology previously used, we calculated the sex-specific medians of coffee intake in our population (free from CVD, cancer, and diabetes) and assigned a value of 1 if consumption was above the sex-specific median $(82.9 \mathrm{~g} / \mathrm{d}$ for women and $84.0 \mathrm{~g} / \mathrm{d}$ for men); otherwise, the score was 0 .

We then obtained a 2-level variable that was included as an additional component in the original MDS, thus originating an MDS "supplemented" with coffee with scores ranging from 0 to 10 ; to allow for comparisons among scores, the scores were entered into risk analyses, scaled by their SD.

The categorical Net reclassification index (NRI) and the integrated discrimination improvement (IDI) (24) were used to quantify the predictive value of the MDS supplemented with coffee over the traditional MDS. To estimate these metrics, the follow-up time was censored at 10 years and the risk categories chosen for NRI calculation were $<10 \%$ and $\geq 10 \%$ (Supplemental Table 4 ).

Finally, we estimated the E-value, a parameter that calculates the minimum strength of association that an unmeasured confounder would need to have with both the exposure and outcome to fully explain away a specific exposure-outcome association (25). 

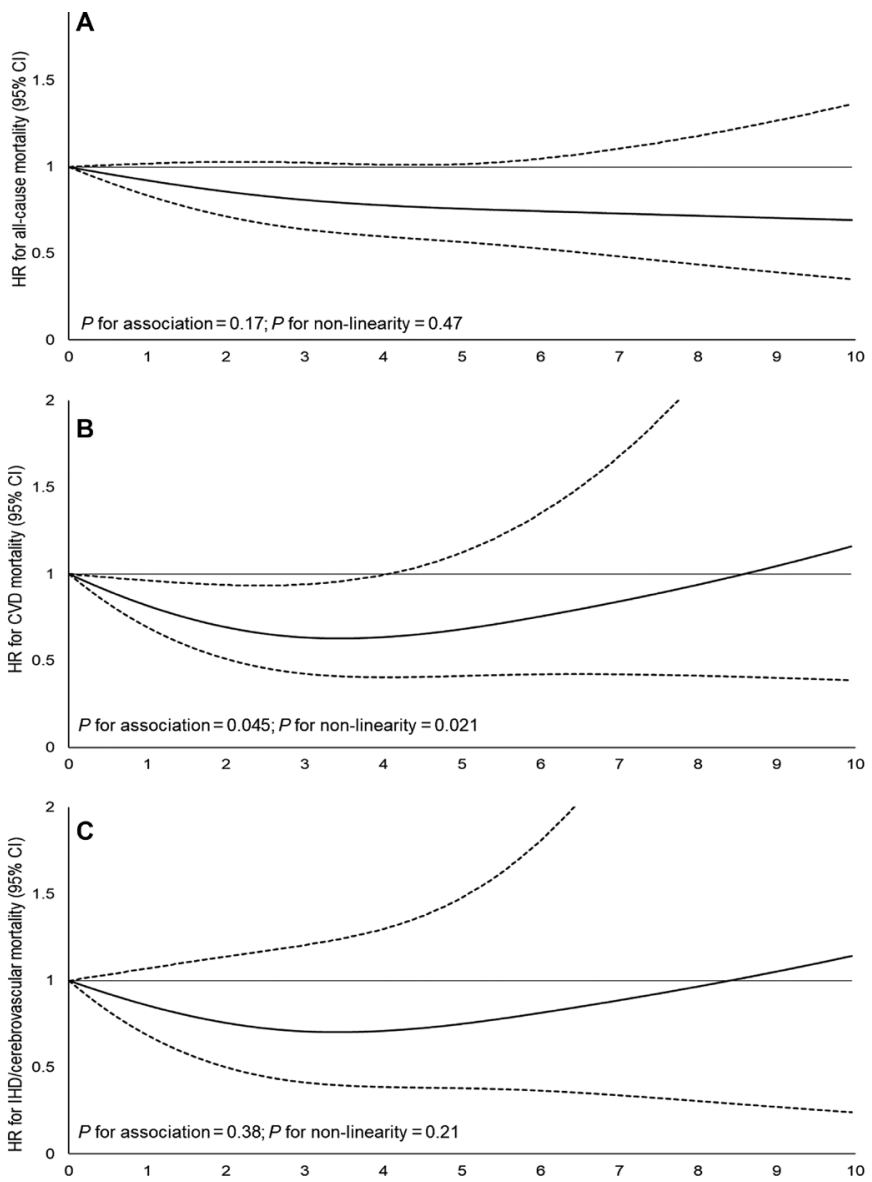

FIGURE 1 Multivariable-adjusted dose-response association of coffee intake (1-cup increment/d) with (A) all-cause, (B) CVD, and (C) IHD/cerebrovascular mortality in the Moli-sani Study cohort ( $n=20,487$ ). Risk estimates (HRs with $95 \% \mathrm{Cls}$ ) were obtained from the multivariable model, adjusted for age, sex, energy intake, education, housing, residence, leisure-time PA, smoking, number of cigarettes per day, abdominal obesity, diabetes, hyperlipidemia, hypertension, Mediterranean diet score, tea intake, and added sugar. The dashed lines represent the $95 \%$ $\mathrm{Cls}$ for the spline model. Participants with no coffee consumption (0 cups/d) served as the reference group. Coffee intake was standardized to a 30-mL Italian espresso cup size. CVD, cardiovascular disease; IHD, ischemic heart disease; PA, physical activity.

The data analysis was generated using SAS/STAT software, Version 9.4, of the SAS System for Windows.

\section{Results}

\section{Characteristics of the population by coffee intake}

In this Moli-sani population, the mean intake of coffee was 2.8 cups per day ( $\mathrm{SD} \pm 1.8)$.

During a median follow up of 8.3 years (IQR, 7.4-9.3 years), 834 subjects died: $270(32.4 \%)$ from CVD, of whom 152 $(18.2 \%)$ died from IHD/cerebrovascular causes; $334(40.0 \%)$ from cancer; and $230(27.6 \%)$ from other causes.
Characteristics of the Moli-sani study population according to daily consumption of coffee are reported in Table 1 . Higher coffee consumers ( $>4$ cups/d; $19.1 \%$ ) tended to be younger and to have a higher level of education and were more likely to be smokers but less likely to have diabetes, hypertension, and hyperlipidaemia; they were also poorly adhering to the MD (Table 1).

\section{Coffee intake and mortality risk}

In multivariable-adjusted Model 2, those who drank $>3$ to $\leq 4$ cups/d had a $28 \%(95 \%$ CI, $8-43 \%)$ lower risk of allcause mortality, as opposed to participants with the lowest consumption (up to $1 \mathrm{cup} / \mathrm{d}$ ), while among heavy consumers 
(>4 cups/d) the mortality risk reduction was no longer significant (Table 2).

When modelling coffee consumption in 1-cup increments per day, the results from main analyses were confirmed $(P$ for association $=0.17 ; P$ for non-linearity $=0.47$; Figure $1 \mathrm{~A}$ ).

Among those participants with habitual intake of $>3$ to $\leq 4$ cups/d of Italian-style coffee, the HRs for CVD and IHD/cerebrovascular mortality were 0.58 (95\% CI, 0.37-0.91) and 0.55 (95\% CI, 0.29-1.03), respectively, while at higher intakes the risk reduction appeared to be lowered (Table 2, Model 2).

Consistently, we found evidence of a nonlinear J-shaped association between coffee consumption and the risk of CVD (magnitude of the relative reduction $=37 \%$; nadir at $3-4 \mathrm{cups} / \mathrm{d} ; P$ value for overall association $=0.045 ; P$ value for nonlinear association $=0.021$; Figure $1 \mathrm{~B}$ ), but not for IHD/cerebrovascular mortality $(P$ value for overall association $=0.38 ; P$ value for nonlinear association $=0.21$; Figure 1C).

No significant inverse associations between the highest $(>4$ cups/d) as opposed to the lowest (up to $1 \mathrm{cup} / \mathrm{d}$ ) coffee intakes were found for cancer death ( $\mathrm{HR}=0.81 ; 95 \% \mathrm{CI}, 0.52-1.26$ ) or mortality from other causes ( $\mathrm{HR}=0.71 ; 95 \% \mathrm{CI}, 0.38-1.31$; Table 2; Model 2).

Survival analyses in the general population of the Molisani Study, including also participants with CVD and cancer at baseline $(n=22,801)$, showed HRs for all-cause, CVD, and $\mathrm{IHD} /$ cerebrovascular death risks of 0.73 (95\% CI, 0.64-0.88), 0.55 (95\% CI, 0.39-0.78), and 0.53 (95\% CI, 0.34-0.84), respectively, for individuals with habitual intake of 3-4 cups/d in comparison with the lowest intake category (Supplemental Table 5).

\section{Mediation analysis}

The associations of coffee intake with all potential mediators (selected biomarkers) are shown in Supplemental Table 1. Coffee intake was positively associated with cardiac troponin, biomarkers of glucose metabolism, total blood cholesterol, and apoB100, whereas it was inversely associated with NTproBNP, HDL, and systolic blood pressure (Supplemental Table 1). The HRs for all-cause, CVD, and IHD/cerebrovascular mortality associated with the selected biomarkers, as estimated by including each biomarker in the multivariable-adjusted model, are shown in Supplemental Table 2. Each of these biomarkers was associated with at least 1 of the outcomes under study, with the exception of insulin and HDL (Supplemental Table 2 ). We finally included in the mediation analysis 5 biomarkers or groups of biomarkers meeting the above mentioned criteria: namely, cardiac troponin, NTproBNP, biomarkers of glucose metabolism, biomarkers of lipid metabolism, and systolic blood pressure. This resulted in a Bonferroni corrected significance threshold $\alpha$ of 0.01 .

A mediation analysis on 18,661 subjects indicated that the majority of established biomarkers of CVD did not substantially modify the relationships between coffee consumption and mortality, with the exception of NTproBNP levels, which explained $11.5 \%(P=0.0052)$ and $26.4 \%(P=0.0006)$ of the relationships between the all-cause mortality risk and coffee consumption of $>3$ to $\leq 4 \mathrm{cups} / \mathrm{d}$ and $>4$ cups/d, respectively (Supplemental Table 6).

The risk reduction of CVD mortality associated with consumption of $>3$ to $\leq 4$ cups/d was slightly accounted for by systolic blood pressure $(3.7 \%$ reduction; $P=0.0078)$, a factor also partially explaining the relationship with the risk of IHD/cerebrovascular death $(5.0 \%$ reduction; $P=0.0032$; Supplemental Table 7).

\section{Additional analyses}

Subgroup analyses are presented in Supplemental Table 3. Coffee intake remained consistently associated with reduced mortality in almost all subgroups. An exception was represented by PA and abdominal obesity, which were likely to modify the magnitude of the inverse associations between coffee consumption and all-cause and CVD mortality risks. Specifically, the associations between a 1-cup increment and all-cause mortality risk were stronger in less physically active subjects $(P$ for interaction $=0.047$ ) and among individuals with abdominal obesity $(P$ for interaction $=0.0068$; Supplemental Table 3 ); those with abdominal obesity were also more likely to have a reduced CVD death risk associated with increased coffee intake, as compared with those participants without abdominal obesity $(P$ for interaction $=0.033)$.

The percentage of decaffeinated coffee consumers was very low in our population sample $(n=741 ; 3.6 \%)$. The mean caffeine intake was $63.8 \mathrm{mg}$ per day and the main caffeine source was coffee; consequently, caffeine intake was highly correlated with the intake of coffee (Spearman correlation coefficients $r=0.80 ; P$ value $<.0001$ ) and represents a strong proxy of it. In addition, to account for other food sources of caffeine, all analyses were also adjusted for tea consumption.

NRI and IDI values indicated that the addition of coffee intake into the MDS did not add to the risk prediction model of the traditional MDS (all $P$ values $>0.05$; Supplemental Table 4).

Finally, by the use of E-values, we found that to nullify the reported association, unobserved confounders should be associated with both coffee consumption and mortality by an $\mathrm{OR} / \mathrm{HR}$ of around 3.0. Of interest, the whole set of known confounders included in our analysis shifted the univariate HR for CVD mortality (HR $=0.29 ; 95 \%$ CI, 0.19-0.44) associated with $>3$ to $\leq 4 \mathrm{cups} / \mathrm{d}$ to the fully adjusted HR of 0.58 (95\% CI, 0.37-0.92; Model 2), acting as a confounder with an E-value of 3.41. Therefore, it is unlikely that 1 (or more) unmeasured factors with an E-value of around 3.0 would exist and create an impact in the way the large, entire set of known confounders considered in our study did.

\section{Discussion}

In a large, adult, Italian population apparently free from major chronic diseases at baseline, a moderate intake of Italian-style coffee was associated with lower risks of allcause and CVD mortality. The association with CVD mortality follows a nonlinear J-shaped curve, showing its nadir at 34 cups per day. This finding is in line with a previous metaanalysis showing evidence of a nonlinear relationship between coffee consumption and CVD mortality, with the strongest association being observed for 3 cups/d (21\% lower risk) (26) and with data from an umbrella review indicating the largest relative risk reduction at intakes of 3 to 4 cups a day versus none (4).

Similarly, a recent meta-analysis on nearly 4 million subjects showed that the lowest relative risks were at intakes of 3.5 cups/d for all-cause mortality and 2.5 cups/d for CVD mortality, while additional intakes were not associated with further reductions in mortality risks (7). 


\begin{tabular}{|c|c|c|c|c|c|}
\hline & \multicolumn{5}{|c|}{ Coffee intake, cups/d } \\
\hline & $\leq 1$ & $>1$ to $\leq 2$ & $>2$ to $\leq 3$ & $>3$ to $\leq 4$ & $>4$ \\
\hline \multicolumn{6}{|l|}{ All-cause mortality, $n=834$} \\
\hline$N$ of deaths/ $n$ of subjects & $245 / 3714$ & $202 / 4436$ & $190 / 4680$ & $108 / 3747$ & $89 / 3910$ \\
\hline Person-years & 30,469 & 36,367 & 38,570 & 31,284 & 32,727 \\
\hline Event rates per 10,000 person-years & 80.4 & 55.5 & 49.3 & 34.5 & 27.2 \\
\hline Model 1 HR $(95 \% \text { CI })^{1}$ & 1.0 & $0.85(0.70-1.02)$ & $0.94(0.78-1.14)$ & $0.86(0.68-1.08)$ & $1.11(0.86-1.43)$ \\
\hline Model 2 HR $\left(95 \%\right.$ CI) ${ }^{2}$ & 1.0 & $0.79(0.65-0.95)$ & $0.84(0.69-1.03)$ & $0.72(0.57-0.92)$ & $0.85(0.62-1.12)$ \\
\hline \multicolumn{6}{|l|}{ CVD mortality, $n=270$} \\
\hline$N$ of deaths/ $n$ of subjects & $92 / 3714$ & $68 / 4436$ & $54 / 4680$ & $27 / 3747$ & $29 / 3910$ \\
\hline Person-years & 30,469 & 36,367 & 38,570 & 31,284 & 32,727 \\
\hline Event rates per 10,000 person-years & 30.1 & 18.7 & 14.0 & 8.6 & 8.9 \\
\hline Model 1 HR $(95 \% \text { CI })^{1}$ & 1.0 & $0.81(0.59-1.11)$ & $0.78(0.55-1.09)$ & $0.66(0.43-1.02)$ & $1.32(0.85-2.06)$ \\
\hline Model 2 HR $(95 \%$ CI) & 1.0 & $0.74(0.54-1.03)$ & $0.70(0.49-0.99)$ & $0.58(0.37-0.91)$ & $1.04(0.62-1.73)$ \\
\hline \multicolumn{6}{|l|}{ IHD/cerebrovascular mortality, $n=152$} \\
\hline$N$ of deaths/ $n$ of subjects & $50 / 3714$ & $35 / 4436$ & $37 / 4680$ & $14 / 3747$ & $16 / 3910$ \\
\hline Person-years & 30,469 & 36,367 & 38,570 & 31,284 & 32,727 \\
\hline Event rates per 10,000 person-years & 16.4 & 9.6 & 9.6 & 4.5 & 4.9 \\
\hline Model $1 \mathrm{HR}(95 \% \mathrm{CI})^{1}$ & 1.0 & $0.78(0.51-1.21)$ & $0.99(0.65-1.53)$ & $0.63(0.35-1.16)$ & $1.37(0.75-2.49)$ \\
\hline Model 2 HR $(95 \% \text { CI })^{2}$ & 1.0 & $0.72(0.46-1.13)$ & $0.90(0.57-1.41)$ & $0.55(0.29-1.03)$ & $1.11(0.56-2.20)$ \\
\hline \multicolumn{6}{|l|}{ Cancer mortality, $n=334$} \\
\hline$N$ of deaths/ $n$ of subjects & $83 / 3714$ & $72 / 4436$ & $85 / 4680$ & $51 / 3747$ & $43 / 3910$ \\
\hline Person-years & 30,469 & 36,367 & 38,570 & 31,284 & 32,727 \\
\hline Event rates per 10,000 person-years & 27.2 & 19.8 & 22.0 & 16.3 & 13.1 \\
\hline Model 1 HR $(95 \% \text { CI })^{1}$ & 1.0 & $0.84(0.61-1.15)$ & $1.13(0.83-1.53)$ & $1.03(0.72-1.46)$ & $1.16(0.79-1.72)$ \\
\hline Model 2 HR $(95 \% \mathrm{CI})^{2}$ & 1.0 & $0.76(0.55-1.06)$ & $0.98(0.71-1.35)$ & $0.82(0.56-1.19)$ & $0.81(0.52-1.26)$ \\
\hline \multicolumn{6}{|l|}{ Other causes mortality, $n=230$} \\
\hline$N$ of deaths $/ n$ of subjects & $70 / 3714$ & $62 / 4436$ & $51 / 4680$ & $30 / 3747$ & $17 / 3910$ \\
\hline Person-years & 30,469 & 36,367 & 38,570 & 31,284 & 32,727 \\
\hline Event rates per 10,000 person-years & 23.0 & 17.0 & 13.1 & 9.6 & 5.2 \\
\hline Model $1 \mathrm{HR}(95 \% \mathrm{Cl})^{1}$ & 1.0 & $0.94(0.67-1.33)$ & $0.92(0.64-1.33)$ & $0.89(0.58-1.37)$ & $0.84(0.48-1.46)$ \\
\hline Model 2 HR $(95 \% \text { CI) })^{2}$ & 1.0 & $0.91(0.64-1.31)$ & $0.85(0.58-1.24)$ & $0.79(0.50-1.26)$ & $0.71(0.38-1.31)$ \\
\hline
\end{tabular}

Data are shown as HRs with 95\% Cls. CVD, cardiovascular disease; IHD, ischemic heart disease; PA, physical activity.

${ }^{1}$ Model 1 is adjusted for age, sex, and energy intake.

${ }^{2}$ Model 2 had the same adjustments as Model 1 and was further controlled for education, housing, residence, leisure-time PA, smoking, number of cigarettes per day,

abdominal obesity, diabetes, hyperlipidemia, hypertension, adherence to Mediterranean diet, tea intake, added sugar.

Potential explanations for the lack of further risk reduction observed for high coffee consumption may rely on the fact that coffee is a composite brew containing several bioactive compounds whose health effects are in opposite directions. Although it is a major source of antioxidants, coffee contains diterpenes, whose concentrations vary by the type of coffee brew, being intermediate in espresso and coffee made in a Moka pot and negligible in drip-filtered, instant, and percolator coffee (27).

Recently, data from the UK Biobank indicated a stronger direct association between blood lipids and espresso coffee as compared to filtered or instant coffee, which is possibly due to the different content of diterpenes with known lipidraising effects (28). However, the effect of caffeine on the cardiovascular system depends on various factors, including the metabolic status and presence of illness (29), and may vary according to different genotypes and gene-environment interactions $(30,31)$.

To the best of our knowledge, this is the first cohort study to analyze the associations of Italian-style coffee and mortality risks in a healthy, adult population. Much of the research so far has evaluated the health effects associated with filtered or boiled coffee, which are the most popular preparation methods in the United States and Scandinavian countries, respectively (32).

The favorable effect of coffee intake on the mortality risk was previously documented by other meta-analyses $(5,26,33)$ and prospective cohort studies (34). Yet, our data on CVD outcomes were not in accordance with prior findings on over 40,000 Italians analyzed in the EPICOR (long-tErm follow-up of antithrombotic management Patterns In acute CORonary syndrome patients) Study, which reported an increased CHD risk associated with consumption of $>2$ cups of Italian-style coffee per day; their results are partially explained by the caffeine content and rapid consumption, resulting in a high peak plasma concentration of caffeine (35).

An umbrella review including meta-analyses of observational studies and randomized controlled trials suggested that coffee may exert beneficial effects on human health, including for some cancers (endometrial, prostate, colorectal, and liver), CVD and metabolic-related outcomes (such as type 2 diabetes and metabolic syndrome), and neurological conditions (such as Parkinson's disease, Alzheimer's disease, and depression) (5). Of note, it was shown in the same Moli-sani population studied here that coffee consumption reduced the risk of prostate cancer (36). 
In EPIC, high coffee consumers ( $>3$ cups per day) had lower all-cause mortality and digestive disease mortality risks compared to nonconsumers (37), in line with results from African Americans, Japanese Americans, Latinos, and Whites in the Multiethnic Cohort (34).

In our analyses, coffee intake was not associated with cancer death, with these results at variance with other population studies $(37,38)$. However, in a Mediterranean setting, a recent study suggested an inverse association between coffee consumption and the cardiovascular mortality risk in elderly (65 years old and above) after a 6-year follow-up, but no effects for total or cancer mortality, in line with our findings (39).

Several potential mechanisms could explain the beneficial effects of moderate coffee consumption on health, including increased blood antioxidant levels (40-43), inhibition of DNA methylation by caffeic acid and chlorogenic acid, 2 common catechol-containing coffee polyphenols (44), or an antiproliferative and antimetastatic activity by caffeine (36). Among other mechanisms, improved insulin sensitivity (45$47)$ and reduced inflammation $(48,49)$ have been suggested as possible pathways by which coffee consumption may reduce the risk of chronic diseases (9). The mechanisms by which higher coffee consumption is associated with increased mortality remain poorly understood.

We tested some known CVD risk factors, reflecting different underlying pathways to disease incidence and progression, as potentially mediating the relationship between coffee consumption and mortality, but failed to find consistent results with the majority of established biomarkers; in contrast, we found that circulating levels of NTproBNP accounted for a substantial proportion of the relationship between coffee consumption and all-cause mortality. NTproBNP is the N-terminal fragment of the B-type natriuretic peptide, secreted by myocytes as a reaction to several stimuli, including wall stretch $(50)$. High levels of NTproBNP have been associated with increased cardiovascular risks and, more recently, with an increased risk of stroke (51); an intervention study on 14 obese, normotensive subjects (52) observed a significant increase in NTproBNP levels after a very short time period of lifestyle intervention, including a dietary intervention with a hypocaloric diet, thus indicating that this natriuretic peptide is susceptible to dietary exposure. We have now observed for the first time that coffee consumption is associated with lower NTproBNP levels, which possibly accounts for the inverse relationship between coffee intake and all-cause mortality. However, our findings should be considered as exploratory and further research is needed to understand the clinical meaning of this association.

We also found that smokers were less likely to experience a CVD mortality risk reduction associated with coffee drinking as compared with nonsmokers, in line with previous studies $(5$, 21).

Lastly, with respect to a potential improvement of risk predictions deriving from the inclusion of coffee consumption into the traditional MDS, we failed to find significant changes in the discrimination ability of the modified score when supplemented with coffee.

The fact that an exposure significantly associated with an outcome does not lead to improvements in predictive value is a well-known and widespread occurrence, and does not entirely devalue the association between the variable and the outcome (53). More importantly, the association of coffee with the mortality risk was independent of the MD, supporting the notion that minor dietary changes, such as consuming coffee regularly within the usual diet, could be valuable measures for improving health, especially cardiovascular health, independent of the overall diet quality.

\section{Strengths and limitations of the study}

The major strengths of this study include a large sample size of an apparently healthy, adult population; a prospective cohort design; and considerable numbers of covariates included for analyses.

Moreover, the robustness of our findings was confirmed by sensitivity analyses. To the best of our knowledge, this is among only a few studies investigating the biological mechanisms potentially linking coffee intake to health outcomes in a primary prevention context.

However, there are also a number of caveats. First, given the observational nature of our investigation, causality can only be suggested and residual confounding cannot be fully ruled out. However, the use of E-values indicates a very small impact of potential unmeasured factors on the strength of our associations. Second, only the all-cause mortality outcome could be fully explored, as cause-specific mortality analyses were limited by the relatively small number of events. Lastly, subjects' information was collected at baseline only; thus, changes that possibly occurred during the follow-up could not be considered.

\section{Conclusions}

In conclusion, this study shows a beneficial effect of Italian-style coffee consumption (when limited to 4 cups per day) on allcause and CVD mortality risks, confirming in a large cohort of Italian adults the results of previous epidemiological studies performed in other settings.

The mechanisms through which moderate coffee consumption could lead to a lower mortality risk are still unclear, although an important mediation was found for NTproBNP levels, while other biomarkers tested were unlikely to be on the coffee-mortality prevention pathway. Finally, the inclusion of coffee intake into a traditional score assessing adherence to the MD did not offer any added value to the discrimination ability of the modified score.

\section{Acknowledgments}

The Moli-sani Study research group thanks the Associazione Cuore Sano Onlus (Campobasso, Italy) for its financial and cultural support, and is grateful to the BiomarCaRE Consortium. The Moli-sani Study Investigators are listed in the Supplementary Appendix.

The authors' responsibilities were as follows-ER, MB, and LI: contributed to the design of the study and interpretation of data; SC, ADeC, and MP: managed data collection; ER, $\mathrm{MB}$, and $\mathrm{ADiC}$ : analyzed the data; ER and MB: wrote the manuscript; MBD, CC, GdG, and LI: originally inspired the research and critically reviewed the manuscript; and all authors: read and approved the final manuscript.

\section{References}

1. Zulli A, Smith RM, Kubatka P, Novak J, Uehara Y, Loftus H, Qaradakhi T, Pohanka M, Kobyliak N, Zagatina A, et al. Caffeine and cardiovascular diseases: critical review of current research. Eur J Nutr 2016;55(4):1331-43.

2. Ferruzzi MG. The influence of beverage composition on delivery of phenolic compounds from coffee and tea. Physiol Behav 2010;100(1):33-41.

3. Ivey KL, Jensen MK, Hodgson JM, Eliassen AH, Cassidy A, Rimm EB. Association of flavonoid-rich foods and flavonoids with risk of all-cause mortality. Br J Nutr 2017;117(10):1470-7. 
4. Poole R, Kennedy OJ, Roderick P, Fallowfield JA, Hayes PC, Parkes J Coffee consumption and health: umbrella review of meta-analyses of multiple health outcomes. BMJ 2017;359:j5024.

5. Grosso G, Godos J, Galvano F, Giovannucci EL. Coffee, caffeine, and health outcomes: an umbrella review. Annu Rev Nutr 2017;37: $131-56$.

6. O'Keefe JH, Bhatti SK, Patil HR, DiNicolantonio JJ, Lucan SC, Lavie CJ. Effects of habitual coffee consumption on cardiometabolic disease, cardiovascular health, and all-cause mortality. J Am Coll Cardiol 2013;62:1043-51.

7. Kim Y, Je Y, Giovannucci E. Coffee consumption and all-cause and cause-specific mortality: a meta-analysis by potential modifiers. Eur J Epidemiol 2019;34(8):731-52.

8. Sc Y, Muralidhara. Beneficial role of coffee and caffeine in neurodegenerative diseases: a minireview. AIMS Public Health 2016;3(2):407-22.

9. Loftfield E, Shiels MS, Graubard BI, Katki HA, Chaturvedi AK, Trabert B, Pinto LA, Kemp TJ, Shebl FM, Mayne ST, et al. Associations of coffee drinking with systemic immune and inflammatory markers. Cancer Epidemiol Biomarkers Prev 2015;24(7):1052-60.

10. Aleksandrova K, Bamia C, Drogan D, Lagiou P, Trichopoulou A, Jenab M, Fedirko V, Romieu I, Bueno-de-Mesquita HB, Pischon T, et al. The association of coffee intake with liver cancer risk is mediated by biomarkers of inflammation and hepatocellular injury: data from the European Prospective Investigation into Cancer and Nutrition. Am J Clin Nutr 2015;102(6):1498-508.

11. Trichopoulou A, Costacou T, Bamia C, Trichopoulos D. Adherence to a Mediterranean diet and survival in a Greek population. N Engl J Med 2003;348(26):2599-608.

12. Di Castelnuovo A, Costanzo S, Persichillo M, Olivieri M, de Curtis A, Zito F, Donati MB, de Gaetano G, Iacoviello L; Molisani Project Investigators. Distribution of short and lifetime risks for cardiovascular disease in Italians. Eur J Prev Cardiol 2012;19: 723-30.

13. Pisani P, Faggiano F, Krogh V, Palli D, Vineis P, Berrino F. Relative validity and reproducibility of a food frequency dietary questionnaire for use in the Italian EPIC centres. Int J Epidemiol 1997;26(Suppl 1):S152-60.

14. Pala V, Sieri S, Palli D, Salvini S, Berrino F, Bellegotti M, Frasca G, Tumino R, Sacerdote C, Fiorini L, et al. Diet in the Italian EPIC cohorts: presentation of data and methodological issues. Tumori 2003;89(6):594-607.

15. Salvini S, Parpinel M, Gnagnarella P, Maissoneuve P, Turrini A. Banca dati composizione degli alimenti per studi epidemiologici in Italia. Milano (Italy): European Institute of Oncology; 1998.

16. The Council for Agricultural Research and Economics, Italy (CREA), Italian Dietary Guidelines. [Internet]. Available from: https://www.crea.gov.it/web/alimenti-e-nutrizione/-/linee-guida-peruna-sana-alimentazione- 2018.

17. WHO Expert Consultation. Waist circumference and waist-hip ratio [Internet]. Geneva (Switzerland): WHO; 8-11 December 2008 [Accessed April 2020]. Available from: http://apps.who.int/iris/bitstrea $\mathrm{m} / 10665 / 44583 / 1 / 9789241501491 \_$_eng.pdf

18. Zeller T, Hughes M, Tuovinen T, Schillert A, Conrads-Frank A Ruijter HD, et al. BiomarCaRE: rationale and design of the European BiomarCaRE project including 300,000 participants from 13 European countries. Eur J Epidemiol 2014;29(10):777-90.

19. Blankenberg S, Zeller T, Saarela O, Havulinna AS, Kee F, Tunstall-Pedoe H, Kuulasmaa K, Yarnell J, Schnabel RB, Wild PS, et al. Contribution of 30 biomarkers to 10 -year cardiovascular risk estimation in 2 population cohorts: the MONICA, risk, genetics, archiving, and monograph (MORGAM) biomarker project. Circulation 2010;121(22): 2388-97.

20. Pounis G, Bonaccio M, Di Castelnuovo A, Costanzo S, de Curtis A, Persichillo M, Sieri S, Donati MB, Cerletti C, de Gaetano G, et al Polyphenol intake is associated with low-grade inflammation, using a novel data analysis from the Moli-sani study. Thromb Haemost. 2015;115(2):344-52.

21. MacKinnon DP, Fairchild AJ, Fritz MS. Mediation analysis. Annu Rev Psychol 2007;58:593-614.

22. Baron RM, Kenny DA. The moderator-mediator variable distinction in social psychological research: conceptual, strategic, and statistical considerations. J Pers Soc Psychol 1986;51:1173-82.
23. Hertzmark E, Pazaris M, Spiegelman D. The SAS MEDIATE macro. Boston (MA): Harvard T.H. Chan School of Public Health; 2012.

24. Pencina MJ, D'Agostino RB, Sr, Steyerberg EW. Extensions of net reclassification improvement calculations to measure usefulness of new biomarkers. Statist Med 2011;30(1):11-21.

25. VanderWeele TJ, Ding P. Sensitivity analysis in observational research: Introducing the e-value. Ann Intern Med 2017;167(4): 268-74.

26. Crippa A, Discacciati A, Larsson SC, Wolk A, Orsini N. Coffee consumption and mortality from all causes, cardiovascular disease, and cancer: a dose-response meta-analysis. Am J Epidemiol 2014;180(8):763-75.

27. van Dam RM, Hu FB, Willett WC. Coffee, caffeine, and health. N Engl J Med 2020;383(4):369-78.

28. Cornelis MC, van Dam RM. Habitual coffee and tea consumption and cardiometabolic biomarkers in the UK Biobank: the role of beverage types and genetic variation. J Nutr 2020;150(10):2772-88.

29. Echeverri D, Montes FR, Cabrera M, Galán A, Prieto A. Caffeine's vascular mechanisms of action. Int $\mathrm{J}$ Vasc Med 2010;2010:834060. doi:10.1155/2010/834060. Erratum in: Int J Vasc Med 2019;2019:7480780.

30. Cornelis MC, El-Sohemy A, Kabagambe EK, Campos H. Coffee, CYP1A2 genotype, and risk of myocardial infarction. JAMA 2006;295(10):1135-41.

31. Palatini P, Ceolotto G, Ragazzo F, Dorigatti F, Saladini F, Papparella I, Mos L, Zanata G, Santonastaso M. CYP1A2 genotype modifies the association between coffee intake and the risk of hypertension. J Hypertens 2009;27(8):1594-601.

32. McCusker RR, Goldberger BA, Cone EJ. Caffeine content of specialty coffees. J Anal Toxicol 2003;27:520-2.

33. Grosso G, Micek A, Godos J, Sciacca S, Pajak A, MartínezGonzález MA, Giovannucci EL, Galvano F. Coffee consumption and risk of all-cause, cardiovascular, and cancer mortality in smokers and non-smokers: a dose-response meta-analysis. Eur J Epidemiol 2016;31(12):1191-205.

34. Park SY, Freedman ND, Haiman CA, Le Marchand L, Wilkens LR, Setiawan VW. Association of coffee consumption with total and causespecific mortality among non-White populations. Ann Intern Med 2017;167(4):228-35.

35. Grioni S, Agnoli C, Sieri S, Pala V, Ricceri F, Masala G, Saieva C, Panico S, Mattiello A, Chiodini P, et al. Espresso coffee consumption and risk of coronary heart disease in a large Italian cohort. PLoS One 2015;10(5):e0126550.

36. Pounis G, Tabolacci C, Costanzo S, Cordella M, Bonaccio M, Rago L, D'Arcangelo D, Filippo Di Castelnuovo A, de Gaetano G, Donati $\mathrm{MB}$, et al. Reduction by coffee consumption of prostate cancer risk: evidence from the Moli-sani cohort and cellular models. Int J Cancer 2017;141(1):72-82.

37. Gunter MJ, Murphy N, Cross AJ, Dossus L, Dartois L, Fagherazzi G, Kaaks R, Kühn T, Boeing H, Aleksandrova K, et al. Coffee drinking and mortality in 10 European countries: a multinational cohort study. Ann Intern Med 2017;167(4):236-47.

38. Sado J, Kitamura T, Kitamura Y, Sobue T, Nishino Y, Tnaka H, Nakayama T, Tsuji I, Ito H, Suzuki T, et al. Association between coffee consumption and all-sites cancer incidence and mortality. Cancer Sci 2017;108(10):2079-87

39. Torres-Collado L, Garcia-de-la-Hera M, Navarrete-Muñoz EM, Notario-Barandiaran L, Gonzalez-Palacios S, Zurriaga O, Melchor I, Vioque J. Coffee consumption and mortality from all causes of death, cardiovascular disease and cancer in an elderly Spanish population. Eur J Nutr 2019;58(6):2439-48.

40. Ding M, Bhupathiraju SN, Satija A, van Dam RM, Hu FB. Long-term coffee consumption and risk of cardiovascular disease: a systematic review and a dose-response metaanalysis of prospective cohort studies. Circulation 2014;129(6):643-59.

41. Moura-Nunes N, Perrone D, Farah A, Donangelo CM. The increase in human plasma antioxidant capacity after acute coffee intake is not associated with endogenous non-enzymatic antioxidant components. Int J Food Sci Nutr 2009;60(Suppl 6):173-81.

42. Mišík M, Hoelzl C, Wagner KH, Cavin C, Moser B, Kundi M, Simic T, Elbling L, Kager N, Ferk F, et al. Impact of paper filtered coffee on oxidative DNA-damage: Results of a clinical trial. Mutat Res 2010;692(1-2):42-8. 
43. Hoelzl C, Knasmüller S, Wagner KH, Elbling L, Huber W, Kager $\mathrm{N}$, Ferk F, Ehrlich V, Nersesyan A, Neubauer O, et al. Instant coffee with high chlorogenic acid levels protects humans against oxidative damage of macromolecules. Mol Nutr Food Res 2010;54(12): 1722-33.

44. Lee WJ, Zhu BT. Inhibition of DNA methylation by caffeic acid and chlorogenic acid, two common catechol-containing coffee polyphenols. Carcinogenesis 2006;27(2):269-77.

45. Arnlöv J, Vessby B, Risérus U. Coffee consumption and insulin sensitivity. JAMA 2004;291(10):1199-1201.

46. Wu T, Willett WC, Hankinson SE, Giovannucci E. Caffeinated coffee, decaffeinated coffee, and caffeine in relation to plasma C-peptide levels, a marker of insulin secretion, in U.S. women. Diabetes Care 2005;28(6):1390-6.

47. Lecoultre V, Carrel G, Egli L, Binnert C, Boss A, MacMillan EL, Kreis R, Boesch C, Darimont C, Tappy L. Coffee consumption attenuates shortterm fructose-induced liver insulin resistance in healthy men. Am J Clin Nutr 2014;99(2):268-75.

48. Lopez-Garcia E, van Dam RM, Qi L, Hu FB. Coffee consumption and markers of inflammation and endothelial dysfunction in healthy and diabetic women. Am J Clin Nutr 2006;84(4):888-93.

49. Kempf K, Herder C, Erlund I, Kolb H, Martin S, Carstensen M, Koenig W, Sundvall J, Bidel S, Kuha S, et al. Effects of coffee consumption on subclinical inflammation and other risk factors for type 2 diabetes: clinical trial. Am J Clin Nutr 2010;91(4):950-7.

50. Di Castelnuovo A, Veronesi G, Costanzo S, Zeller T, Schnabel RB, de Curtis A, Salomaa V, Borchini R, Ferrario M, Giampaoli S, et al. NT-proBNP (N-terminal pro-B-type natriuretic peptide) and the risk of stroke. Stroke 2019;50(3):610-7.

51. Fedele D, Bicchiega V, Collo A, Barutta F, Pistone E, Gruden G, Bruno G. Short term variation in NTproBNP after lifestyle intervention in severe obesity. PLoS One 2017;12(7):e0181212.

52. Daniels LB, Alan S. Maisel. Natriuretic peptides. J Am Coll Cardiol 2007;50:2357-68.

53. Lo A, Chernoff $\mathrm{H}$, Zheng $\mathrm{T}$, Lo $\mathrm{SH}$. Why significant variables aren't automatically good predictors. Proc Natl Acad Sci USA 2015;112:13892-7 
The purpose of this thesis was triple:

1. To re-organize and assess the consumption of major food groups presently included in the Mediterranean diet and its major socioeconomic and psychosocial determinants in a large representative sample of the Italian population recruited in the INHES survey;

2. To evaluate the association with mortality of largely consumed foods and beverages (eggs, chili pepper and coffee), that are not included in the traditional Mediterranean Diet Score, in a large sample of a Mediterranean population recruited in the Moli-sani Study cohort;

3. To assess whether the inclusion of some of these foods/beverages into the traditional Mediterranean Diet Score would improve its risk prediction.

\section{A number of crucial issues have been addressed:}

\section{Two different large study populations}

In the present thesis we used data from two different population cohorts, that is the Italian Nutrition \& Health Survey (INHES study) and the Moli-sani Study, which offered numerous opportunities to address the key purposes of this thesis.

The INHES study is a telephone-based survey on nutrition and health specifically designed to collect information on dietary habits (quality, quantity and patterns), food choice determinants, and food health awareness of the Italian population according to different geographical distribution (Northern, Central and Southern Italy), age, gender and socioeconomic status (1). The sampled subjects were distributed in the four seasons (excluding Christmas, Easter and mid-August periods). The survey calendar was organized to capture an adequate proportion of weekdays and weekend days at group level.

The INHES cohort consists of 9,319 women and men aged $\geq 5$ years recruited from all over Italy, between November 2010 and November 2013. The survey covers the whole Italian territory thus allowing a region-based analysis; moreover, it enrolled children and adolescents for a full coverage of eating habits across all age groups.

Finally, the INHES survey also relies on the use of 24-hours dietary recall and allows a full assessment of eating behaviors/patterns. Of course, the survey shares all the limitations of 
this study type, such as telephone-based collection of data and self-reported medical information. Moreover, data collection is based only on one-day recall.

The Moli-sani Study is a prospective cohort study of 24,325 citizens (men and women aged $\geq 35$ years) from the Molise region, an area placed between Central and Southern Italy, established in 2005-2010 with the purpose of investigating genetic and environmental risk factors in the onset of cardiovascular, cerebrovascular and tumor diseases (2).

The Moli-sani Study is based on face-to-face interviews and clinical examinations, and gained dietary data by using the EPIC questionnaire, a 188-item semi-quantitative food frequency questionnaire, adapted to the Italian population (3).

Owing to its longitudinal design, the Moli-sani cohort offers the opportunity of analyzing prospectively the association between diet and health outcomes.

\section{Up-to-date information on food groups mainly consumed in Italy}

The last effort for an accurate evaluation of dietary habits at a national level in Italy dates back to 2005-06, when INRAN retrieved data from 1329 households corresponding to 3323 women and men (4). Food group consumption data were collected at individual level in 3 consecutive days using hard-copy diaries structured by meal and the data management system INRAN-DIARIO 3.1.

Chapter 2 presented the up-to-date information on food group consumption using data from the INHES survey, that have been collected until very recently (2013). Additionally, and for the very first time, this chapter has addressed the dietary assessment methodologies, using the INHES sample size and the classification of single food items to food groups was performed using the Exposure Hierarchy tree of the European Food Safety Authority's (EFSA's) FoodEx2 (version 2) classification system (5), recently available (April 2015).

Among foods included in the Mediterranean diet pyramid, we focused on whole grain foods due to the growing attention that that these products have gained during the very last years because of their inverse association with disease risk (6-9). The Mediterranean diet pyramid recommends the consumption of one or two servings of cereals at each main meal (in the form of bread, pasta, rice, couscous and others), preferably whole grain (10).

Findings presented in Chapter 3 indicate that whole grain intake is consumed on a regular basis ( $\geq 1$ time/week) by $26.9 \%$ of the Italian study sample, with whole grain bread being the 
major food source of total whole grain food for both age groups (children/adolescents and adults), up to half of the total whole grain intake.

Among major determinants, we highlighted a prominent role of socioeconomic status, with advantaged adult individuals being more likely to report regular intake as compared to lower classes.

Among children/adolescents, living in Southern Italy resulted associated with regular whole grain intake, while among adults, we found that subjects from Northern Italy were likely to consume whole grains more regularly than their counterparts.

We also found that regular whole grain consumers tended to have a healthier lifestyle, including higher physical activity and abstention from tobacco, as already documented in other epidemiological settings (11).

Furthermore, regular whole grain food intake was also associated with some meal patterns and eating-related behaviors: for example, individuals eating meals out of home (e.g. breakfast or lunch) were less likely to consume whole grain food, as well as children/adolescents spending much time watching TV, thus suggesting that whole grain consumption is associated with a healthier eating behavior.

\section{Evaluation of the adherence to the whole Mediterranean diet in Italy and its determinants}

Recent data have shown that adherence to Mediterranean diet has been rapidly declining in Mediterranean countries during the last decades (12). So far, data on dietary habits in the Italian population were mainly obtained from relatively small samples of subjects or pertaining to specific districts, (13) which can likely prevent from an actual estimation of the dietary habits across the whole country, with little chance to offer insights on possible differences at national level. In light of this, Chapter 4 represents the first attempt to identify the major determinants of the Mediterranean diet all over Italy, in recent years, after the beginning of the economic crisis, which has been previously associated with a dramatic drop in the adherence to the Mediterranean diet. First, we found a clear educational gradient in adherence to this eating model, with higher educational attainment being more likely associated with Mediterranean diet as opposed to the lowest educational group. These findings are in accordance with previous analysis from the Moli-sani study (14), highlighting a central role of socioeconomic status (mainly income) in determining the adherence to the Mediterranean diet. 
We also found a number of psychosocial factors being associated with Mediterranean diet, as already documented in the Moli-sani Study population (15), and also that an optimal adherence to Mediterranean diet is closely associated with middle or older age, site of residence (Southern Italy) and higher education level. Finally, findings from Chapter 4 showed that the association of the Mediterranean diet with major demographic (age) and socioeconomic factors (education) was likely to be modified by geographical area. Indeed, adult and older subjects more likely reported high adherence to the Mediterranean diet in Central and Southern regions, rather than in the Northern part of Italy; moreover, living in an urban environment was inversely associated with Mediterranean diet in the Northern part of Italy, and positively in the Central and Southern regions of the country.

Such findings indicate that adherence to the Mediterranean diet seems to be strongly affected by socio-cultural background of Italian individuals, in the Southern regions as part of the population cultural heritage and affordability of prices of the goods, while in the Northern regions as a health-conscious choice to which only the richer part of the population has access.

\section{Intake of fish - even in a processed form - is good for the heart, but should respect food safety rules}

Dietary guidelines recommend eating fish at least two times (two servings) a week (16), because of the well documented inverse association with risk of developing cardiovascular disease supported by several meta-analyses (17).

This hypothesis is further supported by findings from Chapter 5 examining the association between fish intake and risk of composite coronary heart disease (CHD) and stroke in the general Mediterranean population recruited in the Moli-sani Study. We found an inverse association of high fish intake with incidence of composite fatal and non-fatal CHD and stroke. The importance of consuming fish regularly was also highlighted in another investigation from the Moli-sani Study, showing that, among the nine food items, higher fish intake was a major contributor to the overall effect of the Mediterranean Diet Score in reducing mortality risk among the elderly (18).

During the last decades, the opportunity of promoting large fish intake has been highly debated due to the growing public concern about the reported presence of contaminants in fish. 
Considering the nutritional contribution that fish makes to the diet, the European Food Safety Authority's (EFSA) recommended that vulnerable groups in particular select fish from a wide range of species without giving preference to fish types likely containing higher levels of methylmercury, such as swordfish and tuna (19). In a latest statement, the EFSA Committee recommended that each Country should consider its own pattern of fish consumption, especially the species of fish consumed, and carefully assess the risk of exceeding the tolerable weekly intake of methylmercury while obtaining the health benefits from consumption of fish/seafood (20).

\section{Possible implementation of the current Mediterranean Diet Score with hitherto not included substances}

Identifying the optimal diet (or diets) for chronic disease prevention is a public health priority. Traditionally, nutrition research has focused on single nutrients or specific foods, although individuals do not consume nutrients or foods in isolation. Thus, recent nutritional epidemiologic studies have shifted to dietary pattern analysis, which describes the overall diet; the foods, food groups, and nutrients included; their combination and variety; and the frequency and quantity with which they are habitually consumed (21).

Although providing a good assessment of dietary intake, the traditional Mediterranean Diet Score does not include some widely consumed foods and beverages. It follows that the main aim of this thesis was to evaluate the association of some of these foods/beverages with mortality risk and, in some cases, to test whether their inclusion into the Mediterranean Diet Score would improve its risk prediction.

Chapter 6 analyzed the association between egg consumption and mortality risk among the Moli-sani Study participants. We found that egg intake was associated with increased risk of all-cause, cardiovascular mortality and IHD/cerebrovascular mortality in a non-stepwise manner. Furthermore, we tested three dietary nutrients largely present in eggs as possibly mediating the association with mortality. Dietary cholesterol explained up to $20.7 \%$ of the excess of cardiovascular mortality risk associated with the highest egg intake ( $\geq 2$ eggs/week) and accounted for up to $32 \%$ of IHD/cerebrovascular risk mortality associated with moderate egg intake (1-2 egg/week).

Indeed, opinions on the role of eggs in the diet have changed several times over the past few decades, as well as dietary guidelines for egg consumption for general population vary among 
public health agencies. Previously, the Dietary Guidelines for Americans recommended that cholesterol intake be limited to no more than 300 milligrams per day. The 2015 Dietary Guidelines Advisory Committee (DGAC) will not bring forward this recommendation because available evidence shows no appreciable relationship between consumption of dietary cholesterol and serum cholesterol, consistent with the conclusions of the AHA/ACC report. Cholesterol is not a nutrient of concern for overconsumption (22).

However, based on the high cholesterol content, the Mediterranean Diet Foundation and the INRAN (National Institute for Research on Food and Nutrition) recommend up to 4 eggs per week, as a healthy alternative to fish or meat $(23,24)$.

Several studies $(25,26)$ and even meta-analyses $(27)$ have been conducted to investigate the effects of eggs on cardiovascular health, with very different conclusions.

In addition, evidence on the association of egg intake with mortality risk is limited (28-30), especially within Mediterranean countries, where only one study from general population is apparently available (31).

Spices have long been an essential part of the traditional Mediterranean diet, and are placed, along with herbs, at the base of the Mediterranean diet pyramid both for their nutritional properties and as a valuable substitute for salt (32).

Findings from Chapter 7 revealed that regular consumption ( $>4$ times/week) of chili pepper was reported by $24.3 \%$ of the Moli-sani study participants, while $33.7 \%$ declared none/rare intake. Regular consumption was associated with $23 \%$ and $34 \%$ lower risk of all-cause mortality and CVD mortality, respectively, as opposed to none/rare intake, and the magnitude of the association appeared to be stronger for IHD and cerebrovascular disease mortality.

The association of chili peppers with lower mortality risk is independent of the overall quality of the diet, as measured by a traditional Mediterranean diet.

With respect to a potential improvement of risk prediction deriving from the inclusion of chillies into the traditional Mediterranean Diet Score, we found no significant changes in the discrimination ability of the modified score. However, the fact that an explanatory variable significantly correlated with the outcome does not lead to improvements in prediction is a well-known and widespread occurrence, and does not entirely devaluate the association between the variable and the outcome (33). More importantly, the association of chili pepper with mortality risk was independent of the adherence to the Mediterranean Diet, supporting the notion that minor dietary changes, such as adding chilies to usual diet, could be valuable 
measures for improving health, especially cardiovascular health, independently of overall diet quality.

Of notice, this is the first study addressing the association of chillies with mortality in a Mediterranean population, since previous evidence came from China and US $(34,35)$, and to assess possible biological mechanisms underlying such association.

Finally, Chapter 8 reports data on coffee intake in the Moli-sani Study cohort. Coffee is largely consumed in our population, with regular consumers (>3 cups/day) being about $40 \%$ of the population.

In our study higher Italian-style coffee consumption ( $>3$ to $\leq 4 \mathrm{cups} / \mathrm{d}$ ) was associated with $28 \%$ lower risk of all-cause death, while a nonlinear J-shaped association was found with CVD mortality.

As previously described in the same population, the consumption of high coffee (more than 3 cups/day), in comparison to a lower consumption, was associated with a 53\% reduction in prostate cancer risk (36).

The inclusion of coffee consumption as an additional component of the traditional Mediterranean Diet Score did not improve discrimination ability of the model. However, the improved prediction associated with MDS supplement with coffee was little over the model including the traditional MDS

\section{Future perspective}

As every traditional diet, the Mediterranean diet is facing a global food challenge as the global industrial food system is emerging and strongly contributing to a worldwide transition towards eating models possibly associated with obesity and non-communicable diseases (37-39).

Current assessments of Mediterranean diet, as well as of other healthy dietary patterns, are based on principles of traditional nutritional epidemiology, which appear to be insufficient to keep up with a rapidly evolving food system. Indeed, nutritional epidemiology still relies on the use of tools which were not designed to distinguish foods based on industrial processing, nor on the type of farming (organically/conventionally grown).

Moreover, within a context of global industrial food system that favors food exchanges from one Country to another across the globe, it is crucial to understand how foods not included into the Mediterranean pyramid and/or adhesion score, could be integrated into a traditional 
Mediterranean diet. In this study we did not account for 'exotic' foods (e.g. papaya, spices such as curcuma) because the dietary information available in our Moli-sani Study cohort was limited to foods and beverages largely linked to the Italian culinary tradition.

Future nutrition research should consider to collect dietary data on foods that are progressively becoming part of the Mediterranean food tradition in order to see whether their consumption is able to improve or decrease the healthy potential of traditional diets.

In addition, it is noteworthy that traditional diets that feature whole or minimally processed foods and emphasize home-cooking and food preparation, are being rapidly replaced by diets comprising industrially processed and prepared food products $(40,41)$.

These foods are generally cheap, attractive and convenient (42), and some of them are even considered as virtually 'healthy'. It is the case of fish, which is largely recommended especially for its heart benefits, and is generally consumed in the form of fresh, frozen, dried or canned. Future studies will clarify whether different degrees of processing would potentially have different long-term health impacts.

In this thesis, I observed that consumption of canned fish, which is considered as a processed food according to the latest food classifications (42) is associated with a lower, though not significant, risk of cardiovascular disease (Chapter 5).

The actual challenge for nutritional epidemiology would be to discriminate foods not only on the basis of their presumed health benefits, but also based on the degree of food processing. 
1. Pounis G, Bonanni A, Ruggiero E, et al. Food group consumption in an Italian population using the updated food classification system FoodEx2: Results from the Italian Nutrition \& HEalth Survey (INHES) study. Nutr Metab Cardiovasc Dis. 2017;27(4):307-328.

2. Iacoviello L, Bonanni A, Costanzo S, et al. The MOLISANI Project, a randomized, prospective cohort study in the Molise region in Italy; design, rationale and objectives. Italian J Public Health 2007; 4: 110-118.

3. Pisani P, Faggiano F, Krogh V, et al. Relative validity and reproducibility of a food frequency dietary questionnaire for use in the Italian EPIC centres. Int J Epidemiol 1997; 26(Suppl 1):S152e60.

4. Leclercq C, Arcella D, Piccinelli R, et al. The Italian National Food Consumption Survey INRAN-SCAI 2005-06: main results in terms of food consumption. Public Health Nutr 2009;12:2504e32.

5. European Food Safety Authority. Exposure hierarchy FoodEx2 (revision 2). Accessed September 2019

https://www.efsa.europa.eu/sites/default/files/assets/ExposureHierarchyrevision2.z ip.

6. Mellen PB, Walsh TF, Herrington DM. Whole grain intake and cardiovascular disease: a meta-analysis. Nutr Metabol Cardiovasc Dis 2008;18(4):283e90.

7. Iacoviello L, Bonaccio M, Cairella G, et al. Diet and primary prevention of stroke: systematic review and dietary recommendations by the ad hoc Working Group of the Italian Society of Human Nutrition. Nutr Metabol Cardiovasc Dis 2018;28(4):309e34.

8. Montonen J, Knekt P, Järvinen R, et al. Wholegrain and fiber intake and the incidence of type 2 diabetes. Am J Clin Nutr 2003;77(3):622e9.

9. Aune D, Chan DS, Lau R, et al. Dietary fibre, whole grains, and risk of colorectal cancer: systematic review and dose-response meta-analysis of prospective studies. BMJ 2011;10(343):d6617.

10. Bach-Faig A, Berry EM, Lairon D, et al. Mediterranean diet pyramid today. Science and cultural updates. Publ Health Nutr 2011;14(12A):2274e84. 
11. O’Neil CE, Nicklas TA, Zanovec M, Cho S. Whole-grain consumption is associated with diet quality and nutrient intake in adults: the National Health and Nutrition Examination Survey, 1999- 2004. J Am Diet Assoc 2010;110(10):1461e8.

12. Bonaccio M, Bes-Rastrollo $M$, de Gaetano G, Iacoviello L. Challenges to the Mediterranean diet at a time of economic crisis. Nutr Metab Cardiovasc Dis. 2016 Dec;26(12):1057-1063.

13. Grosso G, Marventano S, Giorgianni G, et al. Mediterranean diet adherence rates in Sicily, southern Italy. Public Health Nutr 2014;17:2001-9.

14. Bonaccio M, Bonanni AE, Di Castelnuovo A, et al. Low income is associated with poor adherence to a Mediterranean diet and a higher prevalence of obesity: cross-sectional results from the Moli-sani study. BMJ Open. 2012 Nov 19;2(6). pii: e001685.

15. Bonaccio M, Di Castelnuovo A, Bonanni A, et al. Adherence to a Mediterranean diet is associated with a better health-related quality of life: a possible role of high dietary antioxidant content. BMJ Open. 2013 Aug 13;3(8). pii: e003003.

16. https://www.heart.org/en/healthy-living/healthy-eating/eat-smart/fats/fish-andomega-3-fatty-acids (Accessed September 2019).

17. Zheng J, Huang T, Yu Y, et al. Fish consumption and CHD mortality: an updated metaanalysis of seventeen cohort studies. Public Health Nutr 2012;15:725e37.

18. Bonaccio M, Di Castelnuovo A, Costanzo S, et al. Mediterranean diet and mortality in the elderly: a prospective cohort study and a meta-analysis. Br J Nutr. 2018 Oct;120(8):841-854.

19. https://www.efsa.europa.eu/it/press/news/contam040318 (Accessed September 2019)

20.EFSA Scientific Committee, 2015. Statement on the benefits of fish/seafood consumption compared to the risks of methylmercury in fish/seafood. EFSA Journal 2015:13:3982, 36 pp.

21. Adherence to the traditional Mediterranean diet and mortality in subjects with diabetes. Prospective results from the MOLI-SANI study. Eur J Prev Cardiol. 2016 Mar;23(4):400-7.

22. https://health.gov/dietaryguidelines/2015-scientific-report/pdfs/scientific-report-ofthe-2015-dietary-guidelines-advisory-committee.pdf

23. http://www.fao.org/3/a-as6860.pdf (Accessed September 2019) 
24. Mediterranean Diet Foundation (2010) What's the Mediterranean diet? Internet: http://dietamediterran ea.com/en/nutrition. Accessed 15 Nov 2017

25.Zazpe I, Beunza JJ, Bes-Rastrollo M, et al. Egg consumption and risk of cardiovascular disease in the SUN Project. Eur J Clin Nutr. 2011 Jun;65(6):676-82.

26. Qin C, Lv J, Guo Y, et al. Associations of egg consumption with cardiovascular disease in a cohort study of 0.5 million Chinese adults. Heart. 2018 Nov;104(21):1756-1763.

27. Li Y, Zhou C, Zhou X, Li L. Egg consumption and risk of cardiovascular diseases and diabetes: a meta-analysis. Atherosclerosis. 2013 Aug;229(2):524-30.

28. Xu L, Lam TH, Jiang CQ, et al. Egg consumption and the risk of cardiovascular disease and all-cause mortality: Guangzhou Biobank Cohort Study and meta-analyses. Eur J Nutr. 2019 Mar;58(2):785-796.

29. Nakamura Y, Okamura T, Kita Y, et al. Re-evaluation of the associations of egg intake with serum total cholesterol and cause-specific and total mortality in Japanese women. Eur J Clin Nutr. 2018 Jun;72(6):841-847.

30. Farvid MS, Malekshah AF, Pourshams A, et al. Dietary Protein Sources and All-Cause and Cause-Specific Mortality: The Golestan Cohort Study in Iran. Am J Prev Med. 2017 Feb;52(2):237-248.

31.Zamora-Ros R, Cayssials V, Cleries R, et al. Moderate egg consumption and all-cause and specific-cause mortality in the Spanish European Prospective into Cancer and Nutrition (EPIC-Spain) study. Eur J Nutr. 2019 Aug;58(5):2003-2010.

32. Hu FB. Dietary pattern analysis: a new direction in nutritional epidemiology. Curr Opin Lipidol 2002;13:3-9.

33. Lo A, Chernoff $\mathrm{H}$, Zheng T, Lo SH. Why significant variables aren't automatically good predictors. Proc Natl Acad Sci U S A. 2015;112:13892-7

34. Lv J, Qi L, Yu C, et al. Consumption of spicy foods and total and cause specific mortality: population based cohort study. BMJ. 2015 Aug 4;351:h3942

35. Chopan M, Littenberg B. The Association of Hot Red Chili Pepper Consumption and Mortality: A Large Population-Based Cohort Study. PLoS One. 2017 Jan 9;12(1):e0169876.

36. Pounis G, Tabolacci C, Costanzo S et al. Reduction by coffee consumption of prostate cancer risk: Evidence from the Moli-sani cohort and cellular models. Int J Cancer. 2017 Jul 1;141(1):72-82. 
37. Lawrence MA, Baker PI. Ultra-processed food and adverse health outcomes. BMJ. 2019;365:12289.

38. Poti JM, Braga B, Qin B. Ultra-processed Food Intake and Obesity: What Really Matters for Health-Processing or Nutrient Content? Curr Obes Rep. 2017;6(4):420-31.

39. Monteiro CA, Cannon, G, Levy R, et al. NOVA. The star shines bright. Food classification. Public Health World Nutr. 2016(7):28-38.

40. Poti JM, Braga B, Qin B. Ultra-processed Food Intake and Obesity: What Really Matters for Health-Processing or Nutrient Content? Curr Obes Rep. 2017;6:420-431.

41. Monteiro CA, Cannon G, Levy R, et al. NOVA. The star shines bright. Food classification. Public Health World Nutr 2016;7:28-38.

42. Monteiro CA, Cannon G, Moubarac JC, et al. The UN Decade of Nutrition, the NOVA food classification and the trouble with ultra-processing. Public Health Nutr. 2018 Jan;21(1):5-17. 
The increment of knowledge offered by this thesis over current data, confirms that not only the contribution to health of traditional Mediterranean diet remains intact - when adherence to it is satisfactory - but increases its magnitude, because it adds a surplus-value and is potentiated by other foods or beverages, such as chili pepper and coffee, shown in this thesis to contribute to health and disease risk reduction.

The topic of this thesis is relevant and timely especially in light of the fact that unhealthy diets are major risk factors for a wide range of chronic diseases, including cardiovascular disease, cancer, diabetes as well as all-cause mortality. Specific recommendations for a healthy diet include: eating more fruit, vegetables, legumes, nuts and grains; cutting down on salt, sugar and animal fats (1).

In 2017, 11 million deaths and 255 million disability-adjusted life-years (DALYs) were attributable to dietary risk factors; among them, high intake of sodium (3 million deaths and 70 million DALYs), low intake of whole grains (3 million deaths and 82 million DALYs), and low intake of fruits ( 2 million deaths and 65 million DALYs) were the leading dietary risk factors for deaths and DALYs globally and in many countries (2).

As highlighted in the analysis by the Global Burden of Diseases (2), the Countries that have a diet close to the Mediterranean diet (higher intake of fruits, vegetables, nuts and healthy oils), are the Countries where the lowest number of diet-related deaths were recorded, in line with robust evidence on the lower disease/mortality risk associated with Mediterranean diet, also in non-Mediterranean populations $(3,4)$.

In light of this, preserving a Mediterranean diet, is a major public health task to prolong survival and to reduce risk of major chronic disease.

The first major issue emerging from this thesis is that a number of modifiable factors (e.g. socioeconomic, psychosocial) may influence adherence to this healthy dietary pattern; thus, one of the next challenges nutrition research should address is to reduce food (and health) disparities, also by relying on a socio-ecological model aimed at improving the overall food environment, combined with more efficacious individualized interventions (5).

In this light, recent results from the Moli-sani Study indicated that a person from low socioeconomic status is unlikely to get the same heart advantages of a person with higher income, even in the case that they both similarly adhere to a Mediterranean-like model -and 
get the same traditional dietary score-(6). This observation likely indicates that healthier choices are driven not only by a good set of knowledge and skills but also by greater financial resources. Nutrition literacy and communications are integral to improving diet quality, and health care professionals have an important responsibility to promote healthy eating habits, but often lack basic nutrition knowledge. Therefore, nutrition education and communication programs are recognized as a primary form of intervention in national food and nutrition plans, as they provide people with the knowledge and skills to make healthy dietary and lifestyle choices. Moreover, we should also consider that schools are the ideal setting for promoting healthy eating, because the habits of children and younger people are still being formed (7).

In 2016, an estimated 41 million children under the age of 5 years were overweight or obese, likely due to dietary risk factors including an increased intake of energy-dense foods and a prevalence of physical inactivity (8).

The Moli-sani participants have been repeatedly informed by different means of the results of this thesis (e.g., newsletters, press release, yearly calendar...): in this way, we have ensured that the population involved in our study would consider research activities under a favorable light, thus continuing to collaborate with us and to take part in follow-up procedures.

We're doing it on a regular basis through meetings (called 'I campanili della salute') extended to the general population and organized in different cities and small towns with the main aim of sharing the main findings and discuss the major health issues resulting from the Moli-sani study. A calendar is prepared each year and distributed free of charge to the families of all participants to the study, local general practitioners, public health officers and other selected people.

Within a context of research aiming to identify dietary determinants of health, it was interesting to analyze whether foods and beverages, some already well-known for their health advantages (e.g. coffee), but not included in the traditional Mediterranean Diet Score, nor in the majority of its modified versions, were associated with health outcomes in a population with typical Mediterranean dietary habits.

In this thesis, I observed substantial heart advantages associated with regular consumption of chili pepper (Chapter 7), a spice largely used in the Mediterranean tradition to add flavor to food but also contributing to an important decrease of salt use. 
Based on the effects of high salt intake on blood pressure and on the prominent role of high blood pressure in promoting cardiovascular diseases, a population-wide reduction in salt intake could substantially reduce the incidence of cardiovascular disease (9).

Indeed, reducing salt intake has been identified as one of the most cost-effective measures Countries can take to improve population health outcomes. Moreover, government programs designed to achieve a 10 percent reduction in salt consumption over 10 years could save each year an average of 5.8 million disability-adjusted life years (DALY) (10).

Generally, the promotion of a healthy lifestyle would help reduce the healthcare burdens through lowering the risk of developing multiple chronic diseases, including cancer, cardiovascular disease and diabetes, and extending disease-free life expectancy.

Indeed, adherence to a low risk lifestyle was associated with a longer life expectancy at age 50 , free of major chronic diseases of approximately 7.6 years in men and 10 years in women compared with individuals with no low risk lifestyle factors (11).

Similarly, in the Moli-sani Study, adherence to 4 healthy lifestyle factors, including Mediterranean diet, was associated with higher survival not only within the general population but also among patients at high health risk, such as those with history of CVD or diabetes and among the elderly (12). 
1. https://www.who.int/news-room/fact-sheets/detail/noncommunicable-diseases (Accessed January 2020)

2. GBD 2017 Diet Collaborators. Health effects of dietary risks in 195 countries, 1990 2017: a systematic analysis for the Global Burden of Disease Study 2017. Lancet. 2019 May 11;393(10184):1958-1972.

3. Liyanage T, Ninomiya T, Wang A, et al. Effects of the Mediterranean diet on cardiovascular outcomes - A systematic review and meta-analysis. PLoS One. 2016 Aug 10;11(8):e0159252.

4. Tong T, Wareham NJ, Khaw KT, et al. Prospective association of the Mediterranean diet with cardiovascular disease incidence and mortality and its population impact in a non-Mediterranean population: the EPIC-Norfolk study. BMC Med. 2016 Sep 29;14(1):135.

5. Hu FB, Willett WC. Current and Future Landscape of Nutritional Epidemiologic Research. JAMA. 2018 Nov 27;320(20):2073-2074.

6. Bonaccio M, Di Castelnuovo A, Pounis G, et al. High adherence to the Mediterranean diet is associated with cardiovascular protection in higher but not in lower socioeconomic groups: prospective findings from the Moli-sani study. Int J Epidemiol. 2017;46(5):1478-87.

7. www.fao.org/3/a-a0333e.pdf (Accessed January 2020)

8. https://www.who.int/en/news-room/fact-sheets/detail/obesity-and-overweight (Accessed January 2020)

9. https://www.who.int/dietphysicalactivity/Salt Report VC april07.pdf (Accessed January 2020)

10. Webb M, Fahimi S, Singh GM, et al. Cost effectiveness of a government supported policy strategy to decrease sodium intake: global analysis across 183 nations. BMJ. 2017 Jan 10;356:i6699.

11. Li Y, Schoufour J, Wang DD, et al. Healthy lifestyle and life expectancy free of cancer, cardiovascular disease, and type 2 diabetes: prospective cohort study. BMJ. 2020 Jan 8;368:16669. 
12. Bonaccio M, Di Castelnuovo A, Costanzo S, et al. Impact of combined healthy lifestyle factors on survival in an adult general population and in high-risk groups: prospective results from the Moli-sani Study. J Intern Med. 2019 Aug;286(2):207-220. 

Up to date information on over 70 food groups and subgroups largely consumed in Italy was as a preliminary good source of information for my subsequent nutrition research.

\section{Take-home messages may be summarized as follows:}

1. The Mediterranean diet is strongly affected by socio-cultural background of Italian individuals: in the Southern regions as part of the population cultural heritage and affordability of prices of the goods, while in the Northern regions as a healthconscious choice to which only the richer part of the population has access.

2. The relatively low consumption of whole grain foods observed in a large Italian sample indicates the need of improving public health strategies to increase knowledge and awareness of health benefits associated with whole grain intake.

3. Our findings on fish intake could help promote a regular consumption of fish in line with the European Food Safety Authority's (EFSA) recommendations to select fish from a wide range of species, with reduced preference to fish likely containing higher levels of methylmercury, such as swordfish and tuna.

4. Our findings on egg and mortality risk, along with results from previous population studies, should lead to a more prudent national dietary guideline.

5. Regular chili pepper and coffee consumption could be valuable measures for improving health, especially cardiovascular health. Although adding little to an overall traditional Mediterranean diet from a statistical point of view, they may be well integrated into a Mediterranean-like diet. 

The health benefits associated with the Mediterranean diet have been well established by several epidemiological studies consistently showing that adherence to this dietary pattern is associated with a significant reduction in the risk of developing cardiovascular disease, cancer and neurodegenerative diseases, as well as all-cause mortality.

Nevertheless, the dietary behaviors of many populations have been radically changing since the original definition of this eating pattern and many problematic nutritional aspects emerged, especially within a context of global industrial food system that favors food exchanges from one country to another across the globe.

The Mediterranean Diet Score is the most extensively used index to measure adherence to the Mediterranean diet in epidemiological studies but, although including the key food groups, it leaves out other foods and beverages, some of which might be positively associated with advantageous health outcomes.

This thesis confirmed the health benefits of the traditional Mediterranean diet and its major determinants. Moreover, it addressed the question whether some foods and beverages, neither presently included in the traditional Mediterranean Diet Score, nor in the majority of its modified versions, were associated with health outcomes in a large population with typical Mediterranean dietary habits. The thesis also tested whether the inclusion of these foods and beverages might improve risk prediction beyond a traditional Mediterranean Diet Score. 



\section{Emilia Ruggiero}

\section{Personal details}

- Date of birth: December 24, 1991

- Place of birth: Napoli, Campania, Italy

- Citizenship: Italian

- Civil status: single

- Address: 24 Raffaello Sanzio 86079, Venafro, Italy

- Telephone: +3923334270 (mobile)

- Email: emilia.ruggiero@moli-sani.org

\section{Education}

- 2006-2011: High School at Liceo Scientifico A. Manzoni, Caserta, Italy

- 2011-2014: Bachelor's degree in Health Biotechnology at University of Naples, Napoli, Italy

- 2014-2016: Master's degree in Human Nutrition Science at University of Naples, Napoli, Italy

- June-July 2019 - European Educational Program in Epidemiology - 32nd Residential Summer Course in Epidemiology (Summer 2019, Florence, Italy)

- 2017-2020: PhD student, the Faculty of Health, Medicine and Life Sciences, Maastricht University, The Netherlands.

$\mathrm{PhD}$ thesis title - Mediterranean diet and beyond: an Italian perspective.

\section{Research and working experience}

- 2016-2020: Research fellow in Nutritional Epidemiology at the Department of Epidemiology and Prevention, IRCCS NEUROMED, Pozzilli, Molise, Italy

- 2021: Post-Doctoral Fellowship granted by the Fondazione Umberto Veronesi, Milano, Italy.

\section{Honors and awards}

- XXXVIII National Congress SINU, Turin, Best Oral Communication Award 2017 for the selected scientific contribution: "Socioeconomic and psychosocial determinants of 
adherence to the Mediterranean diet in a general adult Italian population: results from INHES study (Italian Nutrition \& Health Survey).”

- XXXIX National Congress SINU, Naples, Award “Gianvincenzo Barba” 2018 for Scientific Research and Innovation in Human Nutrition: "Socioeconomic and psychosocial determinants of adherence to the Mediterranean diet in a general adult Italian population. European Journal of Public Health (Oxford)".

- EPH Conference Marseille 2019 - Ferenc Bojan session, Best abstract award: "Egg consumption and risk of total and cause-specific mortality in a Mediterranean population"

- 9th European Beer and Health Symposium 2019, Bruxelles, Best abstract award: "Moderate alcohol consumption is associated with reduced low-grade inflammation: results from the Moli-sani Study".

\section{Publications}

- First author of 4 publications

- Co-author of 5 publications as an investigator of the INHES study and Moli-sani Study

- $\quad$ H -Index (calculated via Google scholar) = 7 
1. Pounis G, Bonanni A, Ruggiero E, Di Castelnuovo A, Costanzo S, Persichillo M, Bonaccio M, Cerletti C, Riccardi G, Donati MB, de Gaetano G, Iacoviello L; INHES Investigators. Food group consumption in an Italian population using the updated food classification system FoodEx2: Results from the Italian Nutrition \& HEalth Survey (INHES) study. Nutr Metab Cardiovasc Dis. 2017 Apr; 27(4):307-328. doi: 10.1016/j.numecd.2017.01.004. Epub 2017 Jan 18.

2. Bonaccio M, Ruggiero E, Di Castelnuovo A, Costanzo S, Persichillo M, De Curtis A., Cerletti C, Donati MB, de Gaetano G, Iacoviello L on behalf of the Moli-sani study Investigators. Fish intake is associated with lower cardiovascular risk in a Mediterranean population: prospective results from the Moli-sani study.

Nutr Metab Cardiovasc Dis. 2017 Oct;27(10):865-873. doi: 10.1016/j.numecd.2017.08.004. Epub 2017 Aug 23.

3. Pounis G, Costanzo S, Bonaccio M, Di Castelnuovo A, de Curtis A, Ruggiero E, Persichillo M, Cerletti C, Donati MB, de Gaetano G, Iacoviello L; Moli-sani Investigators. Reduced mortality risk by a polyphenol-rich diet: An analysis from the Moli-sani study. Nutrition. 2018 Apr;48:87-95. doi: 10.1016/j.nut.2017.11.012. Epub 2017 Nov 28.

4. Shivappa N, Bonaccio M, Hebert JR, Di Castelnuovo A, Costanzo S, Ruggiero E, Pounis G, Donati MB, de Gaetano G, Iacoviello L; Moli-sani study Investigators.

Association of proinflammatory diet with low-grade inflammation: results from the Moli-sani study. Nutrition. 2018 Oct;54:182-188. doi: 10.1016/j.nut.2018.04.004. Epub 2018 Apr 21.

5. Ruggiero E, Di Castelnuovo A, Costanzo S, Persichillo M, Bracone F, Cerletti C, Donati MB, de Gaetano G, Iacoviello L, Bonaccio M; INHES Study Investigators.

Socioeconomic and psychosocial determinants of adherence to the Mediterranean diet in a general adult Italian population. Eur J Public Health. 2018 Jul 17. doi: 10.1093/eurpub/cky127.

6. Ruggiero E, Bonaccio M, Di Castelnuovo A, Bonanni A, Costanzo S, Persichillo M, Bracone F, Cerletti C, Donati MB, de Gaetano G, Iacoviello L; INHES Study Investigators. Consumption of whole grain food and its determinants in a general Italian population: Results from the INHES study. Nutr Metab Cardiovasc Dis. 2019 Jun;29(6):611-620. doi: 10.1016/j.numecd.2019.03.001. 
7. Bonaccio M, Di Castelnuovo A, Costanzo S, Ruggiero E, De Curtis A, Persichillo M, Tabolacci C, Facchiano F, Cerletti C, Donati MB, de Gaetano G, Iacoviello L; Moli-sani Study Investigators. Chili Pepper Consumption and Mortality in Italian Adults. J Am Coll Cardiol. 2019 Dec 24;74(25):3139-3149.

8. Ruggiero E, Costanzo S, Di Castelnuovo A, Persichillo M, Esposito S, Magnacca S, Carpineta E, Cerletti C, Bonaccio M, Donati MB, Paolini S, Esposito V, de Gaetano G, Innocenzi $\mathrm{G}$ and Lacoviello $\mathrm{L}$, on behalf of the PREFACE Investigator "Risk Factors for Atherothrombotic Disease and Lumbar Spinal Stenosis: Results from the PREFACE Study." J Spine 9 (2020): 448

9. Angelino D, Rosi A, Ruggiero E, Nucci D, Paolella G, Pignone V, Pellegrini N, Martini D, on behalf of the SINU Young Working Group. Analysis of Food Labels to Evaluate the Nutritional Quality of Bread Products and Substitutes Sold in Italy: Results from the Food Labelling of Italian Products (FLIP) Study. Foods. 2020; 9(12):1905

10. Ruggiero E, Di Castelnuovo A, Costanzo S, Persichillo M, De Curtis A, Cerletti C, Donati MB, de Gaetano G, Iacoviello L, Bonaccio M, on behalf of the Moli-sani Study Investigators. Daily coffee drinking is associated with lower risks of cardiovascular and total mortality in a general Italian population: results from the Moli-sani Study. J Nutr. 2020 Dec 31;nxaa365.

11. Ruggiero E, Di Castelnuovo A, Costanzo S, Persichillo M, De Curtis A, Cerletti C, Donati MB, de Gaetano G, Iacoviello L, Bonaccio M, on behalf of the Moli-sani study Investigators. Egg consumption and risk of total and cause-specific mortality in a healthy Mediterranean population. (Submitted) 
This thesis has been conceived and developed in the framework of the INHES and the Molisani Study that are currently ongoing at the IRCCS Istituto Neurologico Mediterraneo NEUROMED in Pozzilli, to which I'm sincerely grateful.

I am indebted to Professor Hugo ten Cate for accepting me as a $\mathrm{PhD}$ student.

I would like to express my sincere gratitude to my supervisor Marialaura Bonaccio for her continuous support to my PhD study and related research, for her patience, motivation, and large knowledge. Her guidance helped me at all research stages and writing of this thesis.

I am sincerely thankful to Professor Licia Iacoviello whose expertise and guidance actively supported me since the very beginning of my research career.

I would also thank Professor Giovanni de Gaetano and Professor Maria Benedetta Donati, for their time and constructive suggestions, which were determinant for the accomplishment of the work presented in this thesis.

My gratitude goes also to Chiara Cerletti, Augusto Di Castelnuovo and Simona Costanzo and to all my colleagues at the Department of Epidemiology and Prevention, for their companionship and for ensuring a pleasant and friendly working atmosphere.

Last but not least, I wish to thank my mother, my father and my brother for their unconditional support, encouragement and love, without which I would not have come so far.

Finally, I have to thank P. for his continuous encouragement and for always showing how proud he is of me.

\section{Emilia}




